\title{
C*-Quantum Groups with Projection
}

\author{
Dissertation
}

zur Erlangung des mathematisch-naturwissenschaftlichen Doktorgrades

"Doctor rerum naturalium"(Dr.rer.nat.)

der Georg-August-Universität Göttingen

im Promotionsprogramm der PhD School of Mathematical Science (SMS)

der Georg-August University School of Science (GAUSS)

vorgelegt von

Sutanu Roy

aus Kolkata, Indien

Göttingen 2013 
Betreungsausschuss:
Betreuer:
Prof. Dr. Ralf Meyer
Betreuer:
Mathematisches Institut, Georg-August-Universität Göttingen.
Prof. Dr. Dorothea Bahns,
Mathematisches Institut, Georg-August-Universität Göttingen.

Mitglieder der Prüfungskommission:
Referent:
Prof. Dr. Ralf Meyer,
Koreferent:
Mathematisches Institut, Georg-August-Universität Göttingen.
Prof. Dr. Dorothea Bahns,
Mathematisches Institut, Georg-August-Universität Göttingen.

Prof. Dr. Andrea Krajina,

Institut für Mathematische Stochastik, Georg-August-Universität Göttingen.

Prof. Dr. Gert Lube,

Institut für Numerische und Angewandte Mathematik, Georg-August-Universität Göttingen.

Prof. Dr. Karl Henning Rehren,

Institut für Theoretische Physik, Georg-August-Universität Göttingen.

Prof. Dr. Stanisław Lech Woronowicz,

Instytut Matematyki, Uniwersytet w Białymstoku, und, Katedra Metod Matematycznych Fizyki, Wydział Fizyki, Uniwersytet Warszawski.

Tag der mündlichen Prüfung: 26.09.2013 

"what we observe is not nature itself, but nature exposed to our method of questioning."

-Werner Karl Heisenberg

(1901-1976) 

To my parents

Anita and Arun Roy 


\section{Acknowledgement}

At the outset I seek this opportunity to express my gratitude to the people whose support and guidance has been instrumental in making this thesis possible.

Above all I would like to thank profusely my main supervisor Prof. Dr. Ralf Meyer, whose support and guidance gave me the necessary impetus and liberty to pursue this research work. He introduced me to the the theory of quantum groups and motivated me to extend my visionary outlook, the outcome of which is this thesis. It is my utmost fortune that I had the privilege of working with Prof. Dr. Stanisław Lech Woronowicz, whose advice, and unsurpassed knowledge enriched me through and through. His affection and care made all the academic visits to Warsaw even more fruitful, thus a mere thanks does not suffice. The good advice, support and friendship of my second supervisor Prof. Dr. Dorothea Bahns has been invaluable, for which I am extremely grateful.

I would like to thank Deutsche Forchungsgeneimschaft (DFG) for providing me a stipend and generous financial support for my research stays through the Graduiertenkolleg 1493 "Mathematische Strukturen in der modernen Quantenphysik."

I am thankful to the people at the Mathematisches Institut in Göttingen. Apart from the historical significance of the place, the general ambience, the wonderful library, and keenly helpful people provided a congenial climate for research. Special thanks to Johannes Aastrup for the post lunch discussions that were quite enlightening, and also to Manuel Köhler who lucidly explained the preliminary concepts of $\mathrm{C}^{*}$-algebras during my early days in Göttingen.

During the course of my stay I have had the pleasure and privilege of attending seminars and conferences across the globe. I am thoroughly moved by the hospitality that I received from Emmanuel Germain and Roland Vergnioux (Université de Caen), Shrihari Sridharan (CMI, Chennai), Ryszard Nest (Copenhagen), Anupam Saikia and Swaroop Nandan Bora (IIT, Guwahati), Parthasarathy Chakraborty (IMSC, Chennai), Erik Bedos and Sergey Neshveyev (Oslo), Francesca Arici, Ludwik Dąbrowski, Giovanni Landi and Gherardo Piacitelli (SISSA, Trieste), Yasuyuki Kawahigashi (Tokyo), Piotr Hajac, Paweł Kasprzak and Piotr Sołtan (Warsaw).

Last but by no means least, I thank my friends Anupam, Aprameyan, Debranjan, Jannis, Julia, Marc, Rohit, Suliman, Tathagata for their endless support and encouragement throughout my stay in Göttingen. Furthermore, I cannot forget Himadri, Ritwika, Santu back in India, for their advice and support at a crucial stage. My heartfelt thanks to Shamayita for supporting me during the final, critical months of my dissertation. She made me feel like anything is possible.

I extend my sincere gratitude and indebtedness to my parents and my relatives who never made me feel their physical absence. Their patience and unequivocal support throughout helped me remain undaunted in my position, and successfully complete my thesis on time. 



\begin{abstract}
We propose a general theory to study semidirect products of $\mathrm{C}^{*}$-quantum groups in the framework of multiplicative unitaries. Starting from a quantum group with a projection we decompose its multiplicative unitary as a product of two unitary operators. One of them is again a multiplicative unitary in the standard sense; it describes the quotient. The other unitary is multiplicative in a braided sense; it corresponds to the kernel of the projection. Conversely, starting from a standard multiplicative unitary and a braided multiplicative unitary acting on different Hilbert spaces we construct a standard multiplicative unitary acting on the tensor product of them. Basic tools used to achieve this contain the interpretation of bicharacters as homomorphisms between quantum groups, generalised crossed products of $\mathrm{C}^{*}$-algebras carrying coactions of quasitriangular quantum groups (quantum groups with a unitary $R$-matrix), and Yetter-Drinfeld $\mathrm{C}^{*}$-algebras.
\end{abstract}





\section{Contents}

Acknowledgement vii

Abstract $\quad$ ix

\begin{tabular}{llr}
\hline & Introduction & 1
\end{tabular}

\begin{tabular}{|llr}
\hline 2 & $\mathbf{C}^{*}$-quantum groups in a nutshell & $\mathbf{7}$ \\
\hline
\end{tabular}

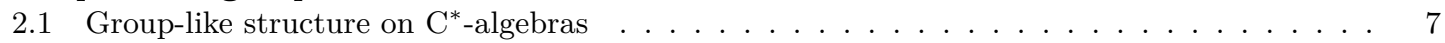

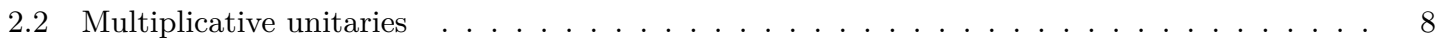

2.2 .1 Manageability and modularity _ . . . . . . . . . . . . . . . . . . . 9

2.3 From modularity to $\mathrm{C}^{*}$-quantum groups . . . . . . . . . . . . . . . . . . . . . . . . 10

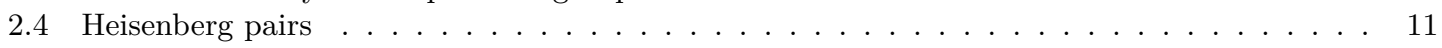

2.5 Coactions of quantum groups $\ldots \ldots \ldots \ldots \ldots \ldots \ldots \ldots$

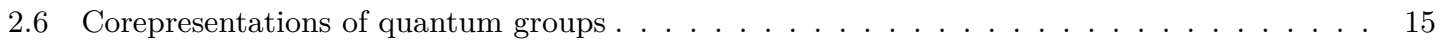

2.7 Universal duals of quantum groups $\ldots \ldots \ldots \ldots \ldots \ldots$

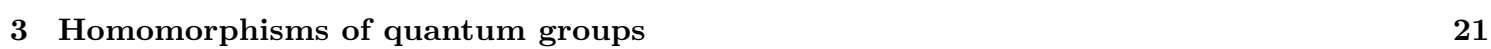

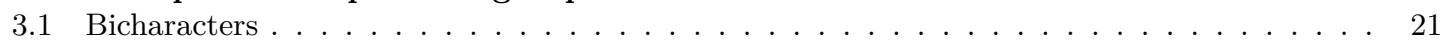

3.1.1 Composition of bicharacters . . . . . . . . . . . . . . . . . . . 22

3.1 .2 Properties of bicharacters . . . . . . . . . . . . . . . . . . . . . . . 23

3.1 .3 Manageability of bicharacters . . . . . . . . . . . . . . . . . . . . . . . . . . 25

3.2 Passage to universal quantum groups . . . . . . . . . . . . . . . . . . 25

3.3 Right and left coactions . . . . . . . . . . . . . . . . . . . . . . . 27

3.4 Functors between coaction categories . . . . . . . . . . . . . . . . . . . . . . . . 30

3.5 Comparison with group homomorphisms . . . . . . . . . . . . . . . . . . . . 33

3.6 Projections on quantum groups $\ldots \ldots \ldots \ldots \ldots \ldots \ldots \ldots$

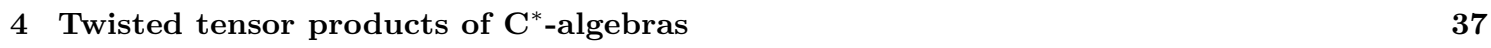

4.1 Heisenberg pairs revisited $\ldots \ldots \ldots \ldots \ldots \ldots \ldots \ldots \ldots$

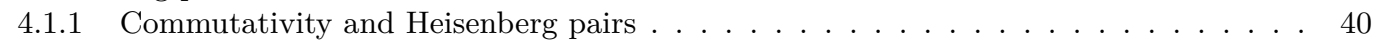

4.2 Twisted tensor products via Heisenberg pairs . . . . . . . . . . . . . . . . . . . . . 4 41

4.3 Hilbert space representation of the twisted tensor product . . . . . . . . . . . . . . . . . . 43

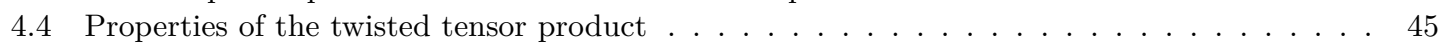

$4.4 .1 \quad$ Functoriality for quantum group morphisms . . . . . . . . . . . . . . . . . . 46

$4.4 .2 \quad$ Functoriality for equivariant morphisms . . . . . . . . . . . . . . . . . . . 47

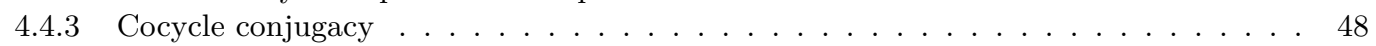

4.5 Examples of twisted tensor products . . . . . . . . . . . . . . . . . . . . 49

$4.5 .1 \quad$ Skew-commutative tensor products $\ldots \ldots \ldots \ldots \ldots \ldots$

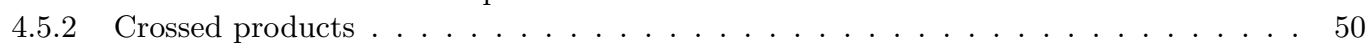

4.6 Generalised quantum codouble and Drinfeld double . . . . . . . . . . . . . . . . . . . . . 52

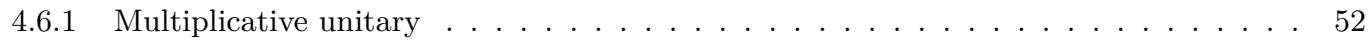

4.6 .2 Generalised Drinfeld doubles . . . . . . . . . . . . . . . . . . . . . . 54 
4.6 .3 Generalised quantum codoubles . . . . . . . . . . . . . . . . 55

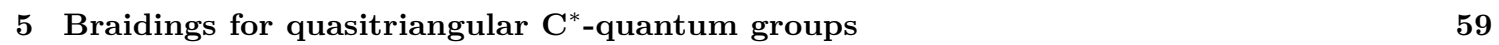

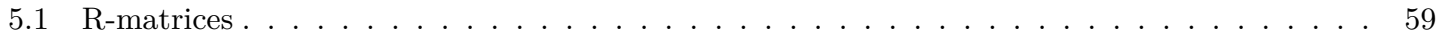

5.1 .1 Universal lift of R-matrices $\ldots \ldots \ldots \ldots \ldots \ldots$

5.2 Quasitriangular $\mathrm{C}^{*}$-quantum groups . . . . . . . . . . . . . . . . . . . . . . 61

5.2 .1 The corepresentation category . . . . . . . . . . . . . . . . . . 61

5.2 .2 Symmetric braidings . . . . . . . . . . . . . . . . . . . . . 63

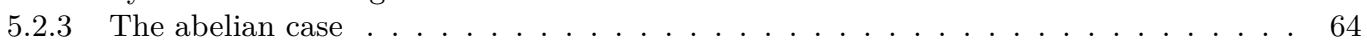

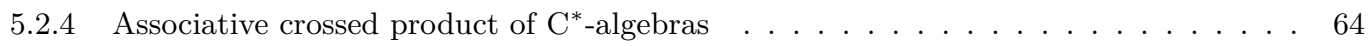

5.3 Quasitriangularity of the quantum codouble . . . . . . . . . . . . . . . . . . . 67

5.3 .1 R-matrix on the Drinfeld double . . . . . . . . . . . . . . . . . . . 68

5.3 .2 Coaction category of the quantum codouble . . . . . . . . . . . . . . . . . 69

5.3 .3 The dual coaction of a quantum group on itself $\ldots \ldots \ldots$. . . . . . . . . . . . . 72

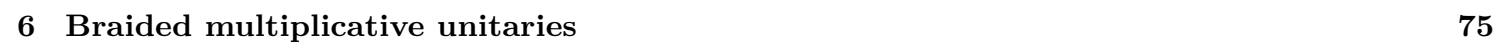

6.1 Braided quantum groups . . . . . . . . . . . . . . . . . . . . . . . . . . . . . . . . . . . . . . . . . .

6.2 Quantum groups with projection . . . . . . . . . . . . . . . . . . . . 78

6.2 .1 Examples of quantum groups with projections . . . . . . . . . . . . . . . . 81

6.2 .2 Quantum $\mathrm{E}(2)$ group $\ldots \ldots \ldots \ldots \ldots \ldots$. . . . . . . . . . . . . . . . . . 82

6.2 .3 Quantum az+b group . . . . . . . . . . . . . . . . . . . . . . . . . . . . 84

6.2 .4 Quantum ax+b group . . . . . . . . . . . . . . . . . . . . . 86

6.3 Construction of a standard multiplicative unitary . . . . . . . . . . . . . . . . . . . 87

6.4 From the complex quantum plane to simplified quantum $\mathrm{E}(2) \ldots \ldots$. . . . . . . . . . . 90

6.4 .1 Preparations on the algebraic level . . . . . . . . . . . . . . . . . . . . . . . 91

$6.4 .2 \quad$ Preparations on the Hilbert space level . . . . . . . . . . . . . . . . . . . . . . . . 92

6.4 .3 Braided quantum group structure on the quantum plane . . . . . . . . . . . . . . . . 94

6.4 .4 Construction of simplified quantum $\mathrm{E}(2) . \ldots \ldots \ldots$. . . . . . . . . . . . 95

$\begin{array}{lr}\text { Bibliography } & 97\end{array}$ 


\section{Chapter 1}

\section{Introduction}

Noncommutative geometry generalises the commutative duality between spaces and functions, in analogy with the Gelfand representation [14] of commutative $\mathrm{C}^{*}$-algebras. Precisely, every commutative $\mathrm{C}^{*}$-algebra is of the form $\mathrm{C}_{0}(X)$ for a locally compact Hausdorff space $X$. Because of this correspondence, the theory of $\mathrm{C}^{*}$-algebras can be realised as noncommutative topology 6 . Hence every $\mathrm{C}^{*}$-algebra can be interpreted as an algebra of functions on a noncommutative space or quantum space. In a similar fashion, von Neumann algebras generalise measure theory in the noncommutative geometry framework: every commutative von Neumann algebra is of the form $\mathrm{L}^{\infty}(X, \mu)$ for a measure space $(X, \mu)$.

\section{$\mathrm{C}^{*}$-quantum groups}

Quantum groups, in the operator algebraic framework, are natural generalisations of locally compact groups within the scope of noncommutative geometry. Roughly speaking, the space of the underlying group is replaced by a $\mathrm{C}^{*}$-algebra or von Neumann algebra, and group operations are replaced by additional structure maps.

The pioneering work of Drinfeld [11] and Jimbo [16] was very influential in the theory of Hopf algebras. In a purely algebraic setting, quantum groups and Hopf algebras are synonymous. Several nice references can be found for algebraic quantum group theory (see [5 20 26]). In [7, 8], van Daele introduced a nonunital generalisation of Hopf algebras which admit a nice duality.

In the $\mathrm{C}^{*}$-algebraic framework, a locally compact group $G$ is described by the $\mathrm{C}^{*}$-algebra $A=\mathrm{C}_{0}(G)$ together with the morphism

$$
\Delta_{\mathrm{C}_{0}(G)}: A \rightarrow A \otimes A, \quad\left(\Delta_{\mathrm{C}_{0}(G)} f\right)(x, y):=f(x y),
$$

for all $f \in \mathrm{C}_{0}(G), x, y \in G$. Motivated by the classical example above, a $\mathrm{C}^{*}$-quantum group $\mathbb{G}$ (or locally compact quantum group in the $\mathrm{C}^{*}$-algebraic framework) should be a pair $\left(A, \Delta_{A}\right)$ consisting of a $\mathrm{C}^{*}$-algebra $A$ and a morphism $\Delta_{A}: A \rightarrow A \otimes A$. Here $\otimes$ denotes the minimal $\mathrm{C}^{*}$-tensor product, and a morphism $A \rightarrow B$ is a non-degenerate ${ }^{*}$-homomorphism from $A$ to the multiplier algebra $\mathcal{M}(B)$. Several assumptions are needed for this to deserve the name $\mathrm{C}^{*}$-quantum group. A simple yet far-reaching axiomatisation of compact quantum groups, generalising compact groups, was given by Woronowicz in 45,48 . In particular, this allows to construct a Haar weight, analogous to the Haar measure on locally compact groups, on compact quantum groups. The most common approaches towards the theory of locally compact quantum groups, due to Kustermans and Vaes 24 25 and Masuda, Nakagami and Woronowicz [27], assume the existence of Haar weights.

In [2], Baaj and Skandalis introduced (regular) multiplicative unitaries in order to formulate an abstract definition of $\mathrm{C}^{*}$-quantum groups. A multiplicative unitary is a unitary operator $\mathbb{W}$ on $\mathcal{H} \otimes \mathcal{H}$, for some Hilbert space $\mathcal{H}$, that satisfies the pentagon equation

$$
\mathbb{W}_{23} \mathbb{W}_{12}=\mathbb{W}_{12} \mathbb{W}_{13} \mathbb{W}_{23} \quad \text { in } \mathcal{U}(\mathcal{H} \otimes \mathcal{H} \otimes \mathcal{H})
$$


A multiplicative unitary simultaneously encodes all the information about a quantum group and its dual. It is still unknown whether a Haar weight can be constructed on a locally compact quantum group arising from a "nice" multiplicative unitary; where nice means either manageable due to Woronowicz [49] or modular by Sołtan and Woronowicz. In this thesis, we work in the general framework of modular or manageable multiplicative unitaries to construct $\mathrm{C}^{*}$-quantum groups.

\section{Semidirect product}

In group theory, the semidirect product is a basic construction generalising direct products. A group $I$ is isomorphic to a semidirect product of groups $K$ and $G$ if and only if there is a (right) split exact sequence:

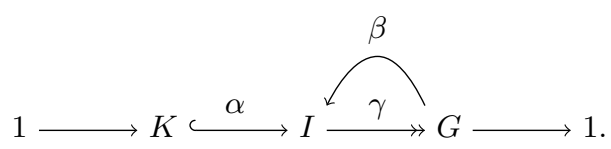

Here exactness means $\operatorname{Im}(\alpha)=\operatorname{Ker}(\gamma)$, while $\gamma \circ \beta=\operatorname{id}_{G}$ is the defining condition for a section. Clearly, $K$ is a normal subgroup of $I$, and $G$ acts on $K$ by conjugation:

$$
\varphi_{g}(k):=\alpha^{-1}\left(\beta(g) \alpha(k) \beta\left(g^{-1}\right)\right)
$$

for $g \in G, k \in K$.

Question. What does a semidirect product decomposition mean for a $\mathrm{C}^{*}$-quantum group?

The multiplication map $K \times K \rightarrow K$ becomes $G$-equivariant with respect to the induced diagonal action of $G$ on $K \times K$. Coactions of $\mathrm{C}^{*}$-quantum groups on $\mathrm{C}^{*}$-algebras generalise group actions on spaces. Roughly speaking, a coaction of $\mathbb{G}$ on a $\mathrm{C}^{*}$-algebra $C$ is an injective morphism $\delta: C \rightarrow C \otimes A$, compatible with the comultiplication map $\Delta_{A}$, and satisfying the Podleś condition (see 2.23 for details). A diagonal coaction, analogous to the diagonal action of groups, makes sense only in the case that $\mathbb{G}$ is abelian or, equivalently, that $A$ is a commutative $\mathrm{C}^{*}$-algebra. This is why we cannot directly generalise 1.1 in the $\mathrm{C}^{*}$-algebraic framework.

The semidirect product always induces an idempotent endomorphism $p:=\beta \circ \gamma$ on $I$. Conversely, an idempotent group homomorphism $p: I \rightarrow I$ is always of the form $(1.1)$, with $G=\operatorname{Im}(p)$ and $K=\operatorname{Ker}(p)$. So a semidirect product decomposition of a group $I$ is equivalent to an idempotent homomorphism on $I$.

To answer the question above, therefore, we view $\mathrm{C}^{*}$-quantum groups with an idempotent endomorphism, briefly, $\mathrm{C}^{*}$-quantum groups with projection, as quantum analogues of semidirect products of groups.

\section{From Hopf algebras with projection to extensions of quantum groups}

Hopf algebras with projection were studied by Radford [35], which justifies viewing Hopf algebras with projection as the correct quantum analogue of semidirect products in the realm of Hopf algebras. The image of the projection is again a Hopf algebra $A$. The analogue of the kernel is a Yetter-Drinfeld algebra $K$ over $A$. For instance, when $A=\mathbb{C}[\mathbb{Z}]$ then $K$ is an $A$-Yetter-Drinfeld algebra if and only if $K$ is a $\mathbb{Z}$-graded $\mathbb{Z}$-module. For two Yetter-Drinfeld algebras $K_{1}$ and $K_{2}$, the tensor product of them, denoted by $K_{1} \otimes K_{2}$ carries a unique multiplication for which it is again a Yetter-Drinfeld algebra; the Yetter-Drinfeld module structure is the diagonal one, which is determined by requiring the embeddings of $K_{1}$ and $K_{2}$ to be equivariant. With this structure, $A$-Yetter-Drinfeld algebras become a monoidal category (neither braided nor symmetric). In a Hopf algebra with projection, the algebra $K$ carries a comultiplication $K \rightarrow K \otimes K$, which turns $K$ into a Hopf algebra in the monoidal category of Yetter-Drinfeld algebras. In 35], Radford shows that Hopf algebras with projection correspond exactly to pairs consisting of a Hopf algebra $A$ and a Hopf algebra in the monoidal category of $A$-Yetter-Drinfeld algebras.

As a result, a semidirect product of Hopf algebras $K \rtimes A$ does not involve two Hopf algebras, but one Hopf algebra $A$ and one "braided" Hopf algebra $K$ over $A$. We want a similar theory for $\mathrm{C}^{*}$-quantum groups. In the case of the semidirect product 1.1 of groups, $K$ and $A$ are identified with $\mathrm{C}_{0}(I / G)$ and 
$\mathrm{C}_{0}(G)$. This clarifies why the "kernel" in Radford's theorem does not correspond to a Hopf algebra in the standard sense.

Associating $\mathrm{C}^{*}$-algebras to these Hopf or Hopf *-algebras requires an interpretation of the defining relations in terms of unbounded operators on Hilbert spaces. This often leads to surprising difficulties. A case in point is the quantum $\mathrm{E}(2)$ studied by Woronowicz in [47. In this example, the comultiplication fails to exist for the most obvious choice of $\mathrm{C}^{*}$-algebra. One must add non-algebraic relations (a restriction on the spectrum of one of the generators) to those that define the Hopf * -algebra in order to get a $\mathrm{C}^{*}$-quantum group.

Moreover, we cannot directly define a deformed multiplication on $K_{1} \otimes K_{2}$. A braided $\mathrm{C}^{*}$-tensor product of $K_{1}$ and $K_{2}$ will be a completion of $K_{1} \otimes K_{2}$ in some $\mathrm{C}^{*}$-norm, but we cannot expect the dense subspace $K_{1} \odot K_{2}$ to be a subalgebra, where $\odot$ denotes the algebraic tensor product. Even the adjoint operation will usually not preserve this subspace.

In the $\mathrm{C}^{*}$-algebra world, the kernel $K$ in Radford's construction corresponds to (functions on) a quantum homogeneous space, which in turn, corresponds to a braided $\mathrm{C}^{*}$-quantum group. Existence of $\mathrm{C}^{*}$-algebraic quantum homogeneous spaces, obtained by taking quotients by closed quantum subgroups, was shown by Vaes 41. Later Kasprzak proposed a more general definition 19, Definition 3.1] of quantum homogeneous spaces. Both constructions of quantum homogeneous spaces use the interplay between $\mathrm{C}^{*}$-algebraic and von Neumann algebraic quantum groups by assuming the existence of Haar weights. The von Neumann algebraic versions of quantum homogeneous spaces were previously used by Vaes and Vainerman 42 to define the short exact sequences ([42, Definition 3.2]) of locally compact quantum groups in order to study their extensions (in von Neumann algebraic framework). The main purpose of this thesis is to study braided multiplicative unitaries as a fundamental object for braided quantum groups and use this to carry over Radford's Theorem to the $\mathrm{C}^{*}$-algebraic framework.

\section{Braided multiplicative unitaries and $\mathrm{C}^{*}$-quantum groups with projection}

We now proceed to sketch our approach towards this, thereby giving an outline of the thesis.

Building on the work by $\mathrm{Ng} 31$ and later by Kustermans 22 we have developed several equivalent notions of quantum group homomorphisms in 28. Bicharacters (see Definition 3.1] can be viewed as homomorphisms and we can also compose them (see Definition 3.6. Thus we can give a good meaning to projections on $\mathrm{C}^{*}$-quantum groups $\mathbb{I}=\left(C, \Delta_{C}\right)$ using bicharacters. Roughly speaking, bicharacters $\mathrm{P} \in \mathcal{U} \mathcal{M}(\hat{C} \otimes C)$ that simultaneously satisfy the pentagon equation are projections. Manageability of $\mathrm{P}$, while viewed as a multiplicative unitary, gets induced from $\mathbb{I}$. Therefore, $\mathrm{P}$ generates a quantum group $\mathbb{G}=\left(A, \Delta_{A}\right)$ which is a closed quantum subgroup of $\mathbb{I}$ in the sense of Woronowicz (see 9, Definition $3.2])$. We call $\mathbb{G}$ the image of the projection $\mathrm{P}$.

For the moment, keeping the general theory aside, let us motivate the interplay between braided multiplicative unitaries and $\mathrm{C}^{*}$-quantum groups with projection, by analysing the known examples of $\mathrm{C}^{*}$-quantum groups, generically denoted as $\mathbb{I}=\left(C, \Delta_{C}\right)$. Multiplicative unitaries giving rise to the quantum $\mathrm{E}(2)$ group by Woronowicz [51, the quantum $a z+b$ group by Woronowicz [50], and the quantum $a x+b$ group by Woronowicz and Zakrzewski $[55$, are of the form $\mathbb{F} \cdot \mathbb{P} \in \mathcal{U}(\mathcal{H} \otimes \mathcal{H})$. All the examples mentioned above were constructed by defoming their respective classical versions, which arise as semidirect product of groups. $\mathbb{P}$ is the unitary corresponding to a projection on $\mathbb{I}$. The image $\mathbb{G}=\left(A, \Delta_{A}\right)$ of the projection $\mathbb{P}$ is commutative. Equivalently, $A=\mathrm{C}_{0}(G)$ for a suitable locally compact group $G$. The unitary $\mathbb{F}$ is defined by using the quantum exponential function (see 52 ); which is rather technical and mysterious. In Section 6.2.1 we show that all these examples are $\mathrm{C}^{*}$-quantum groups with projection. Moreover, $\mathbb{F}$ is a braided multiplicative unitary satisfying a braided variant of the pentagon equation 6.35).

In order to understand the braided quantum group associated to $\mathbb{F}$, we require the concept of YetterDrinfeld $\mathrm{C}^{*}$-algebras over $\mathbb{G}$. The $\mathrm{C}^{*}$-algebraic counterparts of $A$-Yetter-Drinfeld algebras are $\mathbb{G}$-YetterDrinfeld $\mathrm{C}^{*}$-algebras, defined by Nest and Voigt in [30, Section 3] assuming the existence of Haar weights on $\mathbb{G}$. Moreover, 30 . Proposition 3.2] shows that $\mathrm{C}^{*}$-algebras with a coaction of the quantum codouble of $\mathbb{G}$ are the same as $\mathbb{G}$-Yetter-Drinfeld $\mathrm{C}^{*}$-algebras. Proposition 8.3 in $[41$ gives the twisted tensor product $\mathrm{C}^{*}$-algebras, denoted by $\otimes$, such that one of the tensor factors carries a coaction of $\mathbb{G}$ and the other one carries a coaction of $\widehat{\mathbb{G}}$. If both of the $\mathrm{C}^{*}$-algebras are $\mathbb{G}$-Yetter-Drinfeld, so is their twisted tensor product. 
First, we generalise this construction of twisted tensor product of $\mathrm{C}^{*}$-algebras to modular multiplicative

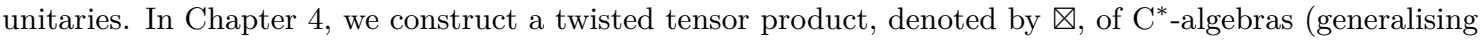
minimal tensor products) endowed with coactions of two quantum groups linked through bicharacters. But $\square$ is not coassociative in general, as there is no natural action of either of the quantum groups on the twisted tensor product. A unitary $\mathrm{U} \in \mathcal{U} \mathcal{M}(\mathbb{K}(\mathcal{H}) \otimes A)$ is called a corepresentation of a quantum group $\mathbb{G}=\left(A, \Delta_{A}\right)$ acting on a Hilbert space $\mathcal{H}$ if $\left(\mathrm{id}_{\mathcal{H}} \otimes \Delta_{A}\right) \mathrm{U}=\mathrm{U}_{12} \mathrm{U}_{13}$. The category of corepresentations of $\mathbb{G}$ is, in general, a $W^{*}$-category (for details see 39 , Section 3.1, 3.2]). A quantum group $\mathbb{G}$ with an $R$-matrix $\mathcal{R} \in \mathcal{U M}(\hat{A} \otimes \hat{A})$ is called a quasitriangular $\mathrm{C}^{*}$-quantum group. Then the corepresentation category of $\mathbb{G}$ becomes braided monoidal. Using covariant representations, we show that the coaction category of $\mathbb{G}$-C $\mathrm{C}^{*}$-algebras, where the objects are $\mathrm{C}^{*}$-algebras carrying coactions of $\mathbb{G}$ and arrows are $\mathbb{G}$-equivariant morphisms, is monoidal with the tensor product $\otimes$ (Theorem 5.16). For any $\mathrm{C}^{*}$-quantum group $\mathbb{G}$ (not necessarily quasitriangular), the Drinfeld double of $\mathbb{G}$ has an $R$-matrix; hence, its dual, the quantum codouble $\mathfrak{D}(\mathbb{G})^{\wedge}$ is a quasitriangular quantum group. By virtue of 30 , Proposition 3.2] we know that the category of $\mathbb{G}$-Yetter-Drinfeld $\mathrm{C}^{*}$-algebras is equivalent to the coaction category of $\mathfrak{D}(\mathbb{G})^{\wedge}$. We show (Proposition 5.36) that the aforementioned categories are also equivalent as monoidal categories.

Replacing the ordinary flip operator by the braiding operator of the corepresentation category of $\mathfrak{D}(\mathbb{G})^{\wedge}$, we can formulate the braided pentagon equation and braided multiplicative unitaries (Definition 6.1) over $\mathbb{G}$. Furthermore, we define manageability for $\mathbb{F}$ (see Definition 6.3). At present, we do not know how to generate $\mathrm{C}^{*}$-algebras even from manageable braided multiplicative unitaries. A pair $\left(K, \Delta_{K}\right)$ is said to be a braided quantum group over $\mathbb{G}$ if $K$ is a $\mathbb{G}$-Yetter-Drinfeld $\mathrm{C}^{*}$-algebra and $\Delta_{K}: K \rightarrow K \otimes K$ is a nondegenerate ${ }^{*}$-homomorphism implemented by $\mathbb{F}$ (see Definition 6.5 for details).

A quantum group $\mathbb{I}$ with a projection $\mathbb{P}$ (Definition 3.35) always gives rise to a manageable, braided multiplicative unitary $\mathbb{F}$ over $\mathbb{G}$, where $\mathbb{G}$ is the image of the projection $\mathbb{P}$ (Theorem 6.7). The second leg of $\mathbb{F}$ belongs to the multiplier algebra of the left invariants under the unique left quantum group homomorphism associated to the projection. Moreover, we can reconstruct a manageable multiplicative unitary of $\mathbb{I}$ starting from $\mathbb{P}$ and $\mathbb{F}$. Similarly, one can define a braided multiplicative unitary $\overline{\mathbb{F}}$ whose second leg belongs to the multiplier algebra of the right invariants under the unique right quantum group homomorphism associated to the projection $\mathbb{P}$.

Starting from a braided multiplicative unitary $\mathbb{F} \in \mathcal{U}(\mathcal{K} \otimes \mathcal{K})$, over a quantum group $\mathbb{G}=\left(A, \Delta_{A}\right)$ with a multiplicative unitary $\mathbb{W}^{A} \in \mathcal{U}(\mathcal{H} \otimes \mathcal{H})$, we construct a standard multiplicative unitary $\mathbb{W}_{1234} \in$ $\mathcal{U}(\mathcal{H} \otimes \mathcal{K} \otimes \mathcal{H} \otimes \mathcal{K})$ (Theorem 6.15). Moreover, manageability of $\mathbb{F}$ ensures manageability of $\mathbb{W}_{1234}$ (Theorem 6.16) and hence gives rise to a quantum group $\mathbb{I}=\left(C, \Delta_{C}\right)$. Under the additional assumption that $\mathbb{G}$ is weakly regular (Definition 5.37), $A$ becomes a $\mathbb{G}$-Yetter-Drinfeld $\mathrm{C}^{*}$-algebra. Let $\mathbb{F}$ give rise to the braided quantum group $\left(K, \Delta_{K}\right)$. Then the multiplicative unitary $\mathbb{W}_{1234}$ implements a coassociative comultiplication on the crossed product $C=A \otimes K$, which extends both $\Delta_{A}$ and $\Delta_{K}$, respectively. In particular, if $A=\mathrm{C}_{0}(G)$ for a locally compact group $G$, then $C=K \rtimes G$; this is true for the known examples of $\mathrm{C}^{*}$-quantum groups discussed in the Section 6.2.1

The missing part of the theory is that, in general, we do not know whether $A \otimes K$ is a quantum group generated by $\mathbb{W}_{1234}$. In particular, the above reconstruction for a $C^{*}$-quantum group $\mathbb{I}$ with a projection $\mathbb{P}$ yields $\mathbb{W}_{1234}$ as a multiplicative unitary for $\mathbb{I}$ (Proposition 6.9. Therefore, at the level of multiplicative unitaries we have a nice generalisation of Radford's theorem. In [54], Woronowicz constructed simplified quantum $\mathrm{E}(2)$ such that quantum $\mathrm{E}(2)$ is the double cover of it. We conclude by constructing simplified quantum $\mathrm{E}(2)$ starting from the complex quantum plane, which is a braided quantum group over the compact quantum group $\mathrm{C}(\mathbb{T})$.

\section{Basic notation}

Following the standard notation the set of natural numbers, integers, real numbers, complex numbers are denoted by $\mathbb{N}$ (excluding zero), $\mathbb{Z}, \mathbb{R}, \mathbb{C}$ respectively.

From now on, all $\mathrm{C}^{*}$-algebras and Hilbert spaces are assumed to be separable and defined over $\mathbb{C}$. Given a Hilbert space $\mathcal{H}$ we let $\mathbb{K}(\mathcal{H})$ and $\mathbb{B}(\mathcal{H})$ be the algebras of compact and bounded operators on $\mathcal{H}$. The group of unitary operators on $\mathcal{H}$ is denoted by $\mathcal{U}(\mathcal{H})$.

The $\mathrm{C}^{*}$-algebra of continuous complex-valued functions on a locally compact Hausdorff space $X$ vanishing at infinity is denoted by $\mathrm{C}_{0}(X)$. 
The multiplier algebra of a $\mathrm{C}^{*}$-algebra $A$ is denoted by $\mathcal{M}(A)$. The group of unitary multipliers of $A$ is denoted by $\mathcal{U}(A)$. For example, $\mathcal{M}(\mathbb{K}(\mathcal{H}))=\mathbb{B}(\mathcal{H})$ and $\mathcal{M}\left(\mathrm{C}_{0}(X)\right)=\mathrm{C}_{\mathrm{b}}(X)$, where $\mathrm{C}_{\mathrm{b}}(X)$ denotes the algebra of complex-valued bounded functions on a locally compact Hausdorff space $X$.

A morphism between two $\mathrm{C}^{*}$-algebras $A$ and $B$ is a nondegenerate *-homomorphism from $A$ to the multiplier algebra $\mathcal{M}(B)$ or, equivalently, a strictly continuous, unital *-homomorphism from $\mathcal{M}(A)$ to $\mathcal{M}(B)$. The set of morphisms from $A$ to $B$ is denoted by $\operatorname{Mor}(A, B)$. $\mathrm{C}^{*}$-algebras with the above morphisms form a category, which we denote by $\mathfrak{C}^{*} \mathfrak{a l g}$.

Let $A$ and $B$ be norm closed subsets of a $\mathrm{C}^{*}$-algebra $C$. Then $A \cdot B$ denotes the closed linear span of the set $\{a b \mid a \in A$ and $b \in B\}$.

We use $\otimes$ both for the minimal tensor product of $\mathrm{C}^{*}$-algebras and the tensor product of Hilbert spaces, which is well understood from the context.

The standard flip operator $\Sigma: \mathcal{H} \otimes \mathcal{K} \rightarrow \mathcal{K} \otimes \mathcal{H}$ for two Hilbert spaces $\mathcal{H}$ and $\mathcal{K}$ is defined as $\Sigma(\xi \otimes \eta):=\eta \otimes \xi$ for all vectors $\xi \in \mathcal{H}, \eta \in \mathcal{K}$. Similarly, the flip morphism $\sigma: A \otimes B \rightarrow B \otimes A$ is defined on the tensor product of $\mathrm{C}^{*}$ algebras $A$ and $B$. For any $t \in \mathcal{M}(A \otimes B)$ we can denote the leg numberings on the level of $\mathrm{C}^{*}$-algebras as $t_{12}:=t \otimes 1_{C} \in \mathcal{M}(A \otimes B \otimes C), t_{23}:=1_{C} \otimes t_{12} \in \mathcal{M}(C \otimes A \otimes B)$ and $t_{13}:=\sigma_{12}\left(t_{23}\right)=\sigma_{23}\left(t_{12}\right) \in \mathcal{M}(A \otimes C \otimes B)$ for the $\mathrm{C}^{*}$-algebras $A, B$ and $C$, respectively. 


\section{Chapter 2}

\section{$\mathrm{C}^{*}$-quantum groups in a nutshell}

This introductory chapter provides a brief introduction to $\mathrm{C}^{*}$-quantum groups. In particular, the interplay between the theory of multiplicative unitaries and $\mathrm{C}^{*}$-quantum groups is covered in Sections 2.2 and 2.3 The definition and the basic properties of Heisenberg pairs for quantum groups are discussed in Section 2.4 Subsequently, in Sections 2.5 and 2.6 we recall the concepts of coactions and corepresentations of $\mathrm{C}^{*}$-quantum groups. In the concluding section, Section 2.7 we explore universal quantum groups and its corepresentation theory. Moreover, we show that universal bicharacters exist in the context of modular multiplicative unitaries.

\subsection{Group-like structure on $\mathrm{C}^{*}$-algebras}

Definition 2.1 (2, Définition 0.1]). A Hopf-C $\mathrm{C}^{*}$-algebra $\left(D, \Delta_{D}\right)$ is a $\mathrm{C}^{*}$-algebra $D$ equipped with a morphism $\Delta_{D}: D \rightarrow D \otimes D$ called comultiplication such that

1. $\Delta_{D}$ is coassociative:

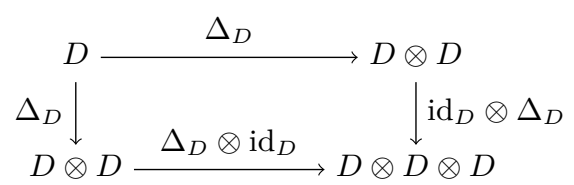

2. $\Delta_{D}(D) \cdot\left(D \otimes 1_{D}\right)$ and $\Delta_{D}(D) \cdot\left(1_{D} \otimes D\right)$ are contained in $D \otimes D$.

Such a pair $\left(D, \Delta_{D}\right)$ is said to be bisimplifiable if $\Delta_{D}$ satisfies the cancellation property:

$$
\Delta_{D}(D) \cdot\left(1_{D} \otimes D\right)=\Delta_{D}(D) \cdot\left(D \otimes 1_{D}\right)=D \otimes D .
$$

In 27] bisimplifiable Hopf $\mathrm{C}^{*}$-algebras are called "proper $\mathrm{C}^{*}$-bialgebras with cancellation property." We shall stick to the former terminology.

Example 2.2. Consider the $\mathrm{C}^{*}$-algebra $\mathrm{C}_{0}(G)$ of continuous functions vanishing at infinity on a locally compact group $G$. Then $\Delta_{\mathrm{C}_{0}(G)}: \mathrm{C}_{0}(G) \rightarrow \mathrm{C}_{\mathrm{b}}(G \times G)$ defined as $\left(\Delta_{\mathrm{C}_{0}(G)} f\right)(x, y):=f(x y)$ for all $f \in \mathrm{C}_{0}(G)$ and $x, y \in G$ is a coassociative comultiplication. To verify the cancellation property take $h \in \mathrm{C}_{0}(G)$ and observe that $\left(\Delta_{\mathrm{C}_{0}(G)}(f)(1 \otimes h)\right)(x, y)=f(x y) h(y),\left(\Delta_{\mathrm{C}_{0}(G)}(f)(h \otimes 1)\right)(x, y)=h(x) f(x y)$. Since $(x, y) \mapsto$ $(x y, y)$ and $(x, y) \mapsto(x y, y)$ are homeomorphisms from $G \times G$ to itself we conclude that $\left(\mathrm{C}_{0}(G), \Delta_{\mathrm{C}_{0}(G)}\right)$ is a bisimplifiable Hopf $\mathrm{C}^{*}$-algebra.

Example 2.3. Let $G$ be a locally compact group with left Haar measure $\mu$. The left regular representation $\lambda: G \rightarrow \mathcal{U}\left(L^{2}(G, \mu)\right)$ is defined by $\left(\lambda_{g} f\right)(x):=f\left(g^{-1} x\right)$ for all $f \in L^{2}(G, \mu), g, x \in G$. The $\mathrm{C}^{*}$-algebra generated by $\lambda\left(\mathrm{C}_{\mathrm{c}}(G)\right) \subset \mathbb{B}\left(L^{2}(G, \mu)\right)$, where $\mathrm{C}_{\mathrm{c}}(G)$ denotes the continuous compactly supported functions, is called the reduced group $\mathrm{C}^{*}$-algebra and denoted by $\mathrm{C}_{\mathrm{r}}^{*}(G)$. Then $\left(\mathrm{C}_{\mathrm{r}}^{*}(G), \Delta_{\mathrm{C}_{\mathrm{r}}^{*}(G)}\right)$ is a bisimplifiable Hopf $\mathrm{C}^{*}$-algebra with the comultiplication $\Delta_{\mathrm{C}_{\mathrm{r}}^{*}(G)}\left(\lambda_{g}\right):=\lambda_{g} \otimes \lambda_{g}$. 
Definition 2.4 (2, Définition 0.5]). Given two Hopf $\mathrm{C}^{*}$-algebras $\left(A, \Delta_{A}\right)$ and $\left(B, \Delta_{B}\right)$, an element $F \in \operatorname{Mor}(A, B)$ is a Hopf ${ }^{*}$-homomorphism between them if it intertwines the comultiplications, that is, the following diagram commutes:

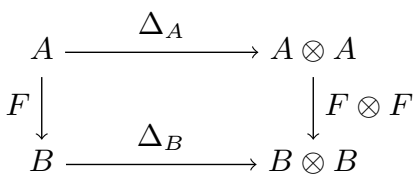

Example 2.5. Let $f: G \rightarrow H$ be a continuous group homomorphism between locally compact groups $G$ and $H$.

1. Then $F: \mathrm{C}_{0}(H) \rightarrow \mathrm{C}_{0}(G)$ defined by $F(h):=h \circ f$ for all $h \in \mathrm{C}_{0}(H)$ is a Hopf *-homomorphism from $\mathrm{C}_{0}(H)$ and $\mathrm{C}_{0}(G)$.

2. If $\operatorname{ker}(f)$ is amenable, then $\hat{F}: \mathrm{C}_{\mathrm{r}}^{*}(G) \rightarrow \mathrm{C}_{\mathrm{r}}^{*}(H)$ defined by $\hat{F}\left(\lambda_{g}\right):=\lambda_{f(g)}$ for all $g \in G$ is a Hopf *-homomorphism from $\mathrm{C}_{\mathrm{r}}^{*}(G)$ to $\mathrm{C}_{\mathrm{r}}^{*}(H)$.

Let $X$ be a locally compact Hausdorff space and let $\left(D, \Delta_{D}\right)$ be a Hopf $\mathrm{C}^{*}$-algebra with $D=\mathrm{C}_{0}(X)$. Then $\Delta_{D}$ induces a continuous, associative binary operation $\cdot$ on $X$. The cancellation property yields that the binary operation - is cancellative; hence $(X, \cdot)$ is a locally compact semigroup with cancellation. If $X$ is compact then $(X, \cdot)$ is a compact group (see $[32)$. More generally, unital, bisimplifiable Hopf $\mathrm{C}^{*}$-algebras $\left(D, \Delta_{D}\right)$ are compact quantum groups, as defined in 48 by Woronowicz. If $X$ is locally compact then $(X, \cdot)$ is not a locally compact group in general. For example, we may take the set of positive integers with addition, which is not group. Therefore, in general, bisimplifiable Hopf $\mathrm{C}^{*}$-algebras are not the correct notion of $\mathrm{C}^{*}$-quantum groups.

The construction of quantum $\mathrm{E}(2)$ group by Woronowicz [51], quantum $a z+b$ group by Woronowicz [50], and the quantum $a x+b$ group by Woronowicz and Zakrzewski [55], in the $\mathrm{C}^{*}$-algebraic framework use the following procedure. The first step is to look at the Hopf *-algebra of polynomial functions on the group in terms of generators and relations. Then deform the relations by some complex number $q$ and consider the ${ }^{*}$-algebra $C$ generated by the generators satisfying the deformed relations. Then define a comultiplication $\Delta_{C}: C \rightarrow C \odot C$ such that $\left(C, \Delta_{C}\right)$ becomes a Hopf ${ }^{*}$-algebra, where $\odot$ is the algebraic tensor product. The second step is to represent the generators of $C$ by (possibly) unbounded closed operators on a Hilbert space $\mathcal{H}$ and impose spectral conditions on the (unbounded) generators of $C$. Equivalently, construct a (universal) $\mathrm{C}^{*}$-algebra $C \subset \mathbb{B}(\mathcal{H})$ generated by the generators (with the spectral conditions) and relations of $C$. The next step is to define the comultiplication on $C$ such that $\left(C, \Delta_{C}\right)$ is a bisimplifiable Hopf-C*-algebra. Finally, construct a multiplicative unitary for $\left(C, \Delta_{C}\right)$. This gives rise to another bisimplifiable Hopf- $\mathrm{C}^{*}$-algebra $\left(\hat{C}, \hat{\Delta}_{C}\right)$, dual to $\left(C, \Delta_{C}\right)$.

But the formula for the multiplicative unitaries for the quantum groups mentioned above uses special functions, namely quantum exponential functions, whose presence is conceptually unclear. Classically, these groups arise as semidirect products of groups. In Chapter 6, we show that quantisation of semidirect products of groups, in general, gives rise to braided multiplicative unitaries, multiplicative unitaries in a more general sense, and quantum exponential functions play this role in the above examples.

\subsection{Multiplicative unitaries}

Multiplicative unitaries were introduced by Baaj and Skandalis in 2$]$ to axiomatise locally compact quantum groups in the $\mathrm{C}^{*}$-algebraic framework. The beauty of the theory is that a single unitary operator (with some additional property) encodes all the necessary information of a $\mathrm{C}^{*}$-quantum group and its dual.

Definition 2.6 ([2, Définition 1.1]). An element $\mathbb{W} \in \mathbb{B}(\mathcal{H} \otimes \mathcal{H})$ is called a multiplicative unitary if it satisfies the pentagon equation

$$
\mathbb{W}_{23} \mathbb{W}_{12}=\mathbb{W}_{12} \mathbb{W}_{13} \mathbb{W}_{23} \quad \text { in } \mathcal{U}(\mathcal{H} \otimes \mathcal{H} \otimes \mathcal{H})
$$

where we use the standard leg numberings $\mathbb{W}_{i j} \in \mathcal{U}(\mathcal{H} \otimes \mathcal{H} \otimes \mathcal{H})$ for $1 \leq i<j \leq 3$. 
Example 2.7. Let $G$ be a locally compact group and let $\mathcal{H}=L^{2}(G)$ be the Hilbert space of all measurable functions on $G$ square-integrable with respect to the right invariant Haar measure. Then $\left(\mathbb{W}^{G} f\right)\left(g, g^{\prime}\right):=$ $f\left(g g^{\prime}, g^{\prime}\right)$ is a unitary oprator on $L^{2}(G \times G)$. The associativity of the binary operation of $G$ provides the pentagon equation 2.4 for $\mathbb{W}^{G}$.

The legs of a multiplicative unitay $\mathbb{W} \in \mathcal{U}(\mathcal{H} \otimes \mathcal{H})$ are defined as

$$
\begin{aligned}
& A:=\left\{(\omega \otimes \mathrm{id}) \mathbb{W}: \omega \in \mathbb{B}(\mathcal{H})_{*}\right\}^{\mathrm{CLS}} \subset \mathbb{B}(\mathcal{H}), \\
& \hat{A}:=\left\{(\mathrm{id} \otimes \omega) \mathbb{W}: \omega \in \mathbb{B}(\mathcal{H})_{*}\right\}^{\mathrm{CLS}} \subset \mathbb{B}(\mathcal{H}),
\end{aligned}
$$

where $\mathbb{B}(\mathcal{H})_{*}$ is the set of normal linear functionals on $\mathbb{B}(\mathcal{H})$ and CLS abbreviates closed linear spans.

Let $a_{i}:=\left(\omega_{i} \otimes \mathrm{id}\right) \mathbb{W} \in A$ for $i=1,2$. Then $a_{1} a_{2}=\left(\omega_{1} \otimes \omega_{2} \otimes \mathrm{id}\right) \mathbb{W}_{13} \mathbb{W}_{23}$. Using the pentagon equation we get $a_{1} a_{2}=\left(\omega_{1} \otimes \omega_{2} \otimes \mathrm{id}\right)\left(\mathbb{W}_{12}^{*} \mathbb{W}_{23} \mathbb{W}_{12}\right)=(\omega \otimes \mathrm{id}) \mathbb{W}$, where $\omega \in \mathbb{B}(\mathcal{H})_{*}$ is defined by $\omega(\xi):=$ $\left(\omega_{1} \otimes \omega_{2}\right)\left(\mathbb{W}^{*}\left(1_{\mathcal{H}} \otimes \xi\right) \mathbb{W}\right)$ for $\xi \in \mathbb{B}(\mathcal{H})$; hence $A$ is a subalgebra of $\mathbb{B}(\mathcal{H})$. A similar argument shows that $\hat{A}$ is a subalgebra of $\mathbb{B}(\mathcal{H})$.

But $A$ and $\hat{A}$ are not closed with respect to the involution in general (see 3, Remark 4.5]). For each $\omega \in$ $\mathbb{B}(\mathcal{H})_{*}$ the adjoint is defined as $a^{*}=((\omega \otimes \mathrm{id}) \mathbb{W})^{*}:=\left(\omega^{*} \otimes \mathrm{id}\right) \mathbb{W}^{*}$ and $\hat{a}^{*}=((\operatorname{id} \otimes \omega) \mathbb{W})^{*}:=\left(\operatorname{id} \otimes \omega^{*}\right) \mathbb{W}^{*}$, where $\omega^{*}: \mathbb{B}(\mathcal{H}) \rightarrow \mathbb{C}$ is defined by $\xi \mapsto \overline{\omega\left(\xi^{*}\right)}$ (see 40, Lemma 7.2.3]). In general, $a^{*}$ and $\hat{a}^{*}$ need not belong to $A$ or $\hat{A}$, respectively. Therefore, a general objective was to find a good class of multiplicative unitaries which produce $A$ in 2.5 and $\hat{A}$ in 2.6 as $\mathrm{C}^{*}$-subalgebras of $\mathbb{B}(\mathcal{H})$.

Define

$$
\mathcal{C}(\mathbb{W}):=\left\{(\operatorname{id} \otimes \omega)(\Sigma \mathbb{W}): \omega \in \mathbb{B}(\mathcal{H})_{*}\right\} \subseteq \mathbb{B}(\mathcal{H}),
$$

where $\Sigma$ is the flip operator on $\mathcal{H} \otimes \mathcal{H}$. Proposition 3.2 in $[2]$ shows that $\mathcal{C}(\mathbb{W})$ is an algebra.

Definition 2.8 (2, Définition 3.3]). A multiplicative unitary $\mathbb{W} \in \mathcal{U}(\mathcal{H} \otimes \mathcal{H})$ is said to be regular if $\mathcal{C}(\mathbb{W})$ is linearly dense in $\mathbb{K}(\mathcal{H})$.

Regularity of a multiplicative unitary implies that $A$ and $\hat{A}$ are $\mathrm{C}^{*}$-algebras (see 2]). But, unfortunately, regularity is too restrictive to cover all examples of locally compact quantum groups (see [1]).

\subsubsection{Manageability and modularity}

A general framework for multiplicative unitaries, namely, manageability, was first introduced in 49 , Definition 1.5]. It covers all known examples of quantum groups. It was also noticed in 38, Section 5] that the natural choices of multiplicative unitaries for the quantum $a z+b$ group in [50] and the quantum $a x+b$ group in 55] are only modular, not manageable.

Notation 2.9. The complex-conjugate Hilbert space of a Hilbert space $\mathcal{H}$ is denoted by $\overline{\mathcal{H}}$.

Definition 2.10 ([38, Definition 2.1]). A multiplicative unitary $\mathbb{W} \in \mathcal{U}(\mathcal{H} \otimes \mathcal{H})$ is modular if there are positive self-adjoint operators $Q$ and $\hat{Q}$ acting on $\mathcal{H}$ and $\widetilde{\mathbb{W}} \in \mathcal{U}(\overline{\mathcal{H}} \otimes \mathcal{H})$ such that:

(i) $\operatorname{Ker}(Q)=\operatorname{Ker}(\hat{Q})=\{0\}$ and $\mathbb{W}(\hat{Q} \otimes Q) \mathbb{W}^{*}=(\hat{Q} \otimes Q)$,

(ii) $(x \otimes u|\mathbb{W}| z \otimes y)=\left(\bar{z} \otimes Q u|\widetilde{\mathbb{W}}| \bar{x} \otimes Q^{-1} y\right)$, for all $x, z \in \mathcal{H}, u \in \mathcal{D o m}(Q)$ and $y \in \mathcal{D} \operatorname{Dom}\left(Q^{-1}\right)$.

If $\hat{Q}=Q$ then $\mathbb{W}$ is called manageable.

The notions of modularity and manageability are not very far from each other: starting from a modular multiplicative unitary one can construct a manageable multiplicative unitary on a different Hilbert space (see 38]). Moreover, all results in 49 remain true under this weaker assumption.

Example 2.11. The multiplicative unitary $\mathbb{W}^{G}$ in Example 2.7 is manageable with $Q=\mathrm{id}$ and $\widetilde{\mathbb{W}}=\left(\mathbb{W}^{G}\right)^{*}$. Here we identify $\mathcal{H}$ with $\overline{\mathcal{H}}$ by sending $f \rightarrow \bar{f}$, where $\bar{f}(g):=f(g)$ for $g \in G$.

Example 2.12. The dual of a multiplicative unitary $\mathbb{W} \in \mathcal{U}(\mathcal{H} \otimes \mathcal{H})$ is defined by $\widehat{\mathbb{W}}:=\Sigma\left(\mathbb{W}^{*}\right) \Sigma \in \mathcal{U}(\mathcal{H} \otimes \mathcal{H})$, where $\Sigma$ is the flip operator. The bidual of a multiplicative unitary is again the multiplicative unitary itself. Moreover, 49, Proposition 2.2] confirms that the duality of multiplicative unitaries preserves modularity and manageability, respectively. 


\subsection{From modularity to $\mathrm{C}^{*}$-quantum groups}

In this section we recall the main result due to Sołtan and Woronowicz 38 to construct a $\mathrm{C}^{*}$-quantum group from a modular multiplicative unitary.

Theorem 2.13 (38, Theorem 2.3]). Let $\mathbb{W} \in \mathcal{U}(\mathcal{H} \otimes \mathcal{H})$ be a modular multiplicative unitary. Then

(1) $A$ in 2.5 and $\hat{A}$ in 2.6 are non-degenerate $\mathrm{C}^{*}$-subalgebras of $\mathbb{B}(\mathcal{H})$.

(2) $\mathbb{W} \in \mathcal{U}(\hat{A} \otimes A) \subseteq \mathcal{U}(\mathcal{H} \otimes \mathcal{H})$. We write $\mathrm{W} \in \mathcal{U}(\hat{A} \otimes A)$ for $\mathbb{W}$ viewed as a unitary multiplier of $\hat{A} \otimes A$.

(3) There is a unique $\Delta_{A} \in \operatorname{Mor}(A, A \otimes A)$ such that

(i) $\left(A, \Delta_{A}\right)$ is a bisimplifiable Hopf $\mathrm{C}^{*}$-algebra;

(ii) $\mathrm{W}$ is a character in the second leg:

$$
\left(\operatorname{id}_{\hat{A}} \otimes \Delta_{A}\right) \mathrm{W}=\mathrm{W}_{12} \mathrm{~W}_{13} \quad \text { in } \mathcal{U}(\hat{A} \otimes A \otimes A) ;
$$

(4) There is a unique closed linear operator $\kappa_{A}$ on the Banach space $A$ such that $\left\{\left(\omega \otimes \mathrm{id}_{A}\right) \mathrm{W}: \omega \in \hat{A}^{\prime}\right\}$ is a core for $\kappa_{A}$ and

for any $\omega \in \hat{A}^{\prime}$. Moreover,

$$
\kappa_{A}\left(\left(\omega \otimes \operatorname{id}_{A}\right) \mathrm{W}\right)=\left(\omega \otimes \operatorname{id}_{A}\right) \mathrm{W}^{*}
$$

(i) the domain of $\kappa_{A}$ is a subalgebra of $A$ and $\kappa_{A}$ is antimultiplicative: for any $a, b \in \mathcal{D}$ om $\left(\kappa_{A}\right)$ we have $a b \in \operatorname{Dom}\left(\kappa_{A}\right)$ and $\kappa_{A}(a b)=\kappa_{A}(b) \kappa_{A}(a)$.

(ii) the image $\kappa_{A}\left(\mathcal{D}\right.$ om $\left.\left(\kappa_{A}\right)\right)$ coincides with $\mathcal{D}$ om $\left(\kappa_{A}\right)^{*}$ and $\kappa_{A}\left(\kappa_{A}(a)^{*}\right)^{*}=$ a for all $a \in \mathcal{D}$ om $\left(\kappa_{A}\right)$.

(iii) the operator $\kappa_{A}$ admits the following polar decomposition:

$$
\kappa_{A}=\mathrm{R}_{A} \circ \tau_{\mathrm{i} / 2}^{A},
$$

where $\tau_{\mathrm{i} / 2}^{A}$ is the analytic generator of a one-parameter group $\left\{\tau_{t}^{A}\right\}_{t \in \mathbb{R}}$ of $*$-automorphisms of the $\mathrm{C}^{*}$-algebra $A$ and $\mathrm{R}_{A}$ is an involutive normal antiautomorphism of $A$,

(iv) $\mathrm{R}_{A}$ commutes with $\tau_{t}^{A}$ for all $t \in \mathbb{R} ;$ in particular $\mathcal{D o m}\left(\kappa_{A}\right)=\operatorname{Dom}\left(\tau_{\mathrm{i} / 2}^{A}\right)$,

(v) $\mathrm{R}_{A}$ and $\left\{\tau_{t}^{A}\right\}_{t \in \mathbb{R}}$ are uniquely determined.

(5) we have

(i) $\Delta_{A} \circ \tau_{t}^{A}=\left(\tau_{t}^{A} \otimes \tau_{t}^{A}\right) \circ \Delta_{A}$ for all $t \in \mathbb{R}$,

(ii) $\Delta_{A} \circ \mathrm{R}_{A}=\sigma \circ\left(\mathrm{R}_{A} \otimes \mathrm{R}_{A}\right) \circ \Delta_{A}$, where $\sigma$ denotes the flip map.

(6) Let $Q$ and $\widetilde{\mathbb{W}}$ be the operators associated to $\mathbb{W}$ in Definition 2.10. Then,

(i) for any $t \in \mathbb{R}$ and $a \in A$ we have $\tau_{t}^{A}(a)=Q^{2 \mathrm{it}} a Q^{-2 \mathrm{it} t}$,

(ii) writing $a^{\mathrm{R}_{A}}$ instead of $\mathrm{R}_{A}(a)$, we have $\mathrm{W}^{\mathrm{\top} \otimes \mathrm{R}_{A}}=\widetilde{\mathbb{W}}^{*}$, where the left hand side is viewed as a unitary on $\overline{\mathcal{H}} \otimes \mathcal{H}$.

Definition 2.14. A bisimplifiable Hopf $\mathrm{C}^{*}$-algebra $\mathbb{G}=\left(A, \Delta_{A}\right)$ is a $\mathrm{C}^{*}$-quantum group (quantum group from now on) if it comes from a modular multiplicative unitary $\mathbb{W} \in \mathcal{U}(\mathcal{H} \otimes \mathcal{H})$.

The reduced dual of $\mathbb{G}$, denoted $\widehat{\mathbb{G}}=\left(\hat{A}, \hat{\Delta}_{A}\right)$, is the quantum group associated to the dual multiplicative unitary $\widehat{\mathbb{W}}^{A}$ in Example 2.12. The biduality property of multiplicative unitaries provides Pontrjagin duality for quantum groups: the reduced dual of $\mathbb{G}$ is isomorphic to $\mathbb{G}$. Moreover, we have the unitary $\widehat{\mathrm{W}}:=\sigma\left(\mathrm{W}^{*}\right) \in \mathcal{U}(A \otimes \hat{A})$. The character condition 2.8 for $\widehat{\mathrm{W}}:=\sigma\left(\mathrm{W}^{*}\right)$, where $\sigma$ is the flip morphism, becomes:

$$
\left(\operatorname{id}_{A} \otimes \hat{\Delta}_{A}\right) \widehat{\mathrm{W}}=\widehat{\mathrm{W}}_{12} \widehat{\mathrm{W}}_{13} \quad \text { in } \mathcal{U}(A \otimes \hat{A} \otimes \hat{A}) .
$$

Using the elementary properties of $\sigma$, we get the following equivalent form:

$$
\left(\hat{\Delta}_{A} \otimes \operatorname{id}_{A}\right) \mathrm{W}=\mathrm{W}_{23} \mathrm{~W}_{13} \quad \text { in } \mathcal{U}(\hat{A} \otimes \hat{A} \otimes A) .
$$

Thus $\mathrm{W} \in \mathcal{U}(\hat{A} \otimes A)$ is also a character in the first leg: 
Definition 2.15 (39). The unitary $\mathrm{W} \in \mathcal{U}(\hat{A} \otimes A)$ is called the reduced bicharacter associated to the quantum group $\mathbb{G}$.

Remark 2.16. The reduced bicharacter of a quantum group is independent of the choice of $\mathbb{W}$ (see 39 Theorem 5]).

To a locally compact group $G$ we associate the quantum groups $\left(\mathrm{C}_{0}(G), \Delta_{\mathrm{C}_{0}(G)}\right)$ and $\left(\mathrm{C}_{\mathrm{r}}^{*}(G), \Delta_{\mathrm{C}_{\mathrm{r}}^{*}(G)}\right)$ discussed in Examples 2.2 and 2.3 The multiplicative unitary $\mathbb{W}^{G} \in \mathcal{U}\left(L^{2}(G \times G)\right)$ defined in Example 2.7 gives rise to $\left(\mathrm{C}_{0}(G), \Delta_{\mathrm{C}_{0}(G)}\right)$, and $\left(\mathrm{C}_{\mathrm{r}}^{*}(G), \Delta_{\mathrm{C}_{\mathrm{r}}^{*}(G)}\right)$ is dual to it. A detailed treatment can be found in 40, Example 7.2.13].

In particular, if $G$ is abelian, $\mathrm{C}_{\mathrm{r}}^{*}(G) \cong \mathrm{C}_{0}(\hat{G})$ by the Fourier transform, where $\hat{G}$ is the Pontrjagin dual of $G$. This clarifies how $\mathrm{C}^{*}$-quantum groups allow to generalise the Pontrjagin duality theorem.

\subsection{Heisenberg pairs}

In quantum mechanics the canonical commutation relation (abbreviated as CCR) is the fundamental relation between position $x$ and momentum $p_{x}$ in the direction of $x$ of a particle. The commutator $\left[x, p_{x}\right]:=x p_{x}-p_{x} x$ is equal to $\mathrm{i} \hbar$, where $\hbar$ is the reduced Planck constant. According to the mathematical formulation of quantum mechanics, $x$ and $p_{x}$ should be represented as self adjoint operators $\mathbf{x}$ and $\mathbf{p}$ on a Hilbert space $\mathcal{H}$, and the commutation relation becomes:

$$
[\mathbf{x}, \mathbf{p}]=\mathrm{i} \hbar \cdot 1_{\mathcal{H}} .
$$

This is known as CCR in Heisenberg form. But 2.10) is not quite correct, as both the operators $\mathbf{x}$ and p cannot be bounded. Hence 2.10 becomes more delicate as unbounded operators are very sensitive about their domains. Exponentiation of the operators ix, ip produces the one-parameter group of unitaries $\left(u_{s}:=\exp (\mathrm{i} s \mathbf{x})\right)_{s \in \mathbb{R}},\left(v_{t}:=\exp (\mathrm{i} t \mathbf{p})\right)_{t \in \mathbb{R}}$ acting on $\mathcal{H}$, and 2.10 takes the Weyl form:

$$
u_{s} v_{t}=\exp (-\mathrm{i} \hbar s t) v_{t} u_{s}
$$

The Stone-von Neumann Theorem says that there is a unique pair of one-parameter groups of unitaries $\left(u_{s}\right)_{s \in \mathbb{R}}$ and $\left(v_{t}\right)_{t \in \mathbb{R}}$ satisfying 2.11). More generally, the CCR encodes the commutation relation between two conjugate variables, where one variable is the Fourier transformation or more generally Pontrjagin dual of another.

Heisenberg pairs in the context of quantum groups are hidden in the pentagon equation 2.4. Roughly, it dictates the commutation between the underlying $\mathrm{C}^{*}$-algebras of a quantum group and its dual. Later in Section 4.1 we generalise this commutation relation to two different quantums group related by a bicharacter.

Definition 2.17. Let $\pi \in \operatorname{Mor}\left(A, \mathbb{K}\left(\mathcal{H}_{\pi}\right)\right)$ and $\hat{\pi} \in \operatorname{Mor}\left(\hat{A}, \mathbb{K}\left(\mathcal{H}_{\pi}\right)\right)$ for some Hilbert space $\mathcal{H}_{\pi}$. $(\pi, \hat{\pi})$ is a

1. $\mathbb{G}$-Heisenberg pair if

$$
\mathrm{W}_{\hat{\pi} 3} \mathrm{~W}_{1 \pi}=\mathrm{W}_{1 \pi} \mathrm{W}_{13} \mathrm{~W}_{\hat{\pi} 3} \quad \text { in } \mathcal{U}\left(\hat{A} \otimes \mathbb{K}\left(\mathcal{H}_{\pi}\right) \otimes A\right)
$$

2. $\mathbb{G}$-anti-Heisenberg pair if

$$
\mathrm{W}_{1 \pi} \mathrm{W}_{\hat{\pi} 3}=\mathrm{W}_{\hat{\pi} 3} \mathrm{~W}_{13} \mathrm{~W}_{1 \pi} \quad \text { in } \mathcal{U}\left(\hat{A} \otimes \mathbb{K}\left(\mathcal{H}_{\pi}\right) \otimes A\right) ;
$$

here $\mathrm{W}_{1 \pi}:=\left(\left(\operatorname{id}_{\hat{A}} \otimes \pi\right) \mathrm{W}\right)$ and $\mathrm{W}_{\hat{\pi} 2}:=\left(\left(\hat{\pi} \otimes \operatorname{id}_{A}\right) \mathrm{W}\right)$ with the leg numbers 1,2 and 3 assigned to $\hat{A}, \mathbb{K}\left(\mathcal{H}_{\pi}\right)$ and $A$, respectively.

Example 2.18. Let $\mathbb{W} \in \mathcal{U}(\mathcal{H} \otimes \mathcal{H})$ be a modular multiplicative unitary which gives rise to the quantum group $\mathbb{G}=\left(A, \Delta_{A}\right)$. Let $\mathrm{W} \in \mathcal{U}(\hat{A} \otimes A)$ be the reduced bicharacter. By Theorem 2.13 (1) there are faithful representations $\pi$ and $\hat{\pi}$ of $A$ and $\hat{A}$ on $\mathcal{H}$ with $\mathbb{W}=(\hat{\pi} \otimes \pi) \mathrm{W} \in \mathcal{U}(\mathcal{H} \otimes \mathcal{H})$. Moreover, considering the preimage of the pentagon equation 2.4 under the injective map $(\hat{\pi} \otimes \mathrm{id} \otimes \pi)$ gives 2.12 as follows:

$$
\mathrm{W}_{\hat{\pi} 3} \mathrm{~W}_{1 \pi}=\mathrm{W}_{1 \pi} \mathrm{W}_{13} \mathrm{~W}_{\hat{\pi} 3} \quad \text { in } \mathcal{U}(\hat{A} \otimes \mathbb{K}(\mathcal{H}) \otimes A) .
$$


Conversely, a pair of faithful 1 representations $(\pi, \hat{\pi})$ of $A$ and $\hat{A}$ on $\mathcal{H}$ is a $\mathbb{G}$-Heisenberg pair if and only if $\mathrm{W}_{\hat{\pi} \pi} \in \mathcal{U}(\mathcal{H} \otimes \mathcal{H})$ is a multiplicative unitary.

The next example shows that $\mathbb{G}$-anti-Heisenberg pairs exist.

Example 2.19. Let $(\pi, \hat{\pi})$ be a $\mathbb{G}$-Heisenberg pair on $\mathcal{H}_{\pi}$ and let $\mathrm{T}: \mathbb{B}\left(\mathcal{H}_{\pi}\right) \rightarrow \mathbb{B}\left(\overline{\mathcal{H}}_{\pi}\right)$ be the transposition defined as $\xi^{\top}(\bar{v}):=\overline{\xi^{*} v}$ for all $v \in \mathcal{H}_{\pi}$, where $\overline{\mathcal{H}}_{\pi}$ is the complex-conjugate Hilbert space. Define

$$
\bar{\pi}(a):=\left(\pi\left(\mathrm{R}_{A}(a)\right)\right)^{\top} \quad \text { and } \quad \overline{\hat{\pi}}(\hat{a}):=\left(\hat{\pi}\left(\mathrm{R}_{\hat{A}}(\hat{a})\right)\right)^{\top}
$$

for all $a \in A, \hat{a} \in \hat{A}$ and $\mathrm{R}_{A}$, with $\mathrm{R}_{\hat{A}}$ as in Theorem 2.13 Then $(\bar{\pi}, \overline{\hat{\pi}})$ is a $\mathbb{G}$-anti-Heisenberg pair acting on $\overline{\mathcal{H}}_{\pi}$ :

$$
\mathrm{W}_{1 \bar{\pi}} \mathrm{W}_{\overline{\bar{\pi}} 3}=\left(\mathrm{R}_{\hat{A}} \otimes \mathrm{T} \otimes \mathrm{R}_{A}\right)\left(\mathrm{W}_{\hat{\pi} 3} \mathrm{~W}_{1 \pi}\right)=\left(\mathrm{R}_{\hat{A}} \otimes \mathrm{T} \otimes \mathrm{R}_{A}\right)\left(\mathrm{W}_{1 \pi} \mathrm{W}_{13} \mathrm{~W}_{\hat{\pi} 3}\right)=\mathrm{W}_{\overline{\bar{\pi}} 3} \mathrm{~W}_{13} \mathrm{~W}_{1 \bar{\pi}},
$$

where the first equality uses [2.15), $\left(\mathrm{R}_{\hat{A}} \otimes \mathrm{R}_{A}\right) \mathrm{W}=\mathrm{W}\left(\left[39\right.\right.$, Lemma 40]) and antimultiplicativity of $\mathrm{R}_{A}$, $\mathrm{R}_{\hat{A}}$ and $\mathrm{T}$, the second equality uses 2.12 , and the third equality follows by applying the facts used for the first equality in reverse order.

Remark 2.20. Example 2.19 gives a one-to-one correspondence between $\mathbb{G}$-Heisenberg and -anti-Heisenberg pairs.

Proposition 2.21. Every $\mathbb{G}$-Heisenberg pair or $\mathbb{G}$-anti-Heisenberg pair is faithful.

First we establish the following lemma.

Lemma 2.22. Let $(\pi, \hat{\pi})$ and $(\rho, \hat{\rho})$ be a $\mathbb{G}$-Heisenberg pair and a $\mathbb{G}$-anti-Heisenberg pair on Hilbert spaces $\mathcal{H}_{\pi}$ and $\mathcal{H}_{\rho}$, respectively. Then $\pi \otimes \hat{\rho}: A \otimes \hat{A} \rightarrow \mathbb{B}\left(\mathcal{H}_{\pi} \otimes \mathcal{H}_{\rho}\right)$ and $\rho \otimes \hat{\pi}: A \otimes \hat{A} \rightarrow \mathbb{B}\left(\mathcal{H}_{\rho} \otimes \mathcal{H}_{\pi}\right)$ are unitarily equivalent.

Proof. Define $\Psi:=\mathrm{W}_{\hat{\rho} \pi} \Sigma \mathrm{W}_{\hat{\pi} \rho} \in \mathcal{U}\left(\mathcal{H}_{\pi} \otimes \mathcal{H}_{\rho}, \mathcal{H}_{\rho} \otimes \mathcal{H}_{\pi}\right)$, where $\mathrm{W}_{\hat{\pi} \rho}:=(\hat{\pi} \otimes \rho) \mathrm{W} \in \mathcal{U}\left(\mathcal{H}_{\pi} \otimes \mathcal{H}_{\rho}\right)$, $\mathrm{W}_{\hat{\rho} \pi}:=(\hat{\rho} \otimes \pi) \in \mathcal{U}\left(\mathcal{H}_{\rho} \otimes \mathcal{H}_{\pi}\right)$ and $\Sigma: \mathcal{H}_{\pi} \otimes \mathcal{H}_{\rho} \rightarrow \mathcal{H}_{\rho} \otimes \mathcal{H}_{\pi}$ is the flip operator. We claim that $\Psi$ intertwines $\pi \otimes \hat{\rho}$ and $\rho \otimes \hat{\pi}$. Using (2.5) and 2.6), it suffices to show:

$$
\Psi_{23} \mathrm{~W}_{1 \pi} \mathrm{W}_{\hat{\rho} 4} \Psi_{23}^{*}=\mathrm{W}_{1 \rho} \mathrm{W}_{\hat{\pi} 4} \quad \text { in } \mathcal{U}\left(\hat{A} \otimes \mathbb{K}\left(\mathcal{H}_{\rho}\right) \otimes \mathbb{K}\left(\mathcal{H}_{\pi}\right) \otimes A\right),
$$

or, equivalently,:

$$
\Sigma_{23}\left(\mathrm{~W}_{\hat{\pi} \rho} \mathrm{W}_{1 \pi} \mathrm{W}_{\hat{\rho} 4}\left(\mathrm{~W}_{\hat{\pi} \rho}\right)^{*}\right) \Sigma_{23}=\left(\mathrm{W}_{\hat{\rho} \pi}\right)^{*} \mathrm{~W}_{1 \rho} \mathrm{W}_{\hat{\pi} 4} \mathrm{~W}_{\hat{\rho} \pi}
$$

in $\mathcal{U}\left(\hat{A} \otimes \mathbb{K}\left(\mathcal{H}_{\rho}\right) \otimes \mathbb{K}\left(\mathcal{H}_{\pi}\right) \otimes A\right)$.

The following computation yields 2.16:

$$
\begin{aligned}
\Sigma_{23}\left(\mathrm{~W}_{\hat{\pi} \rho} \mathrm{W}_{1 \pi} \mathrm{W}_{\hat{\rho} 4}\left(\mathrm{~W}_{\hat{\pi} \rho}\right)^{*}\right) \Sigma_{23} & =\Sigma_{23}\left(\mathrm{~W}_{1 \pi} \mathrm{W}_{1 \rho} \mathrm{W}_{\hat{\pi} \rho} \mathrm{W}_{\hat{\rho} 4}\left(\mathrm{~W}_{\hat{\pi} \rho}\right)^{*}\right) \Sigma_{23} \\
& =\mathrm{W}_{1 \pi} \mathrm{W}_{1 \rho} \mathrm{W}_{\hat{\rho} 4} \mathrm{~W}_{\hat{\pi} 4} \\
& =\left(\mathrm{W}_{\hat{\rho} \pi}\right)^{*} \mathrm{~W}_{1 \rho} \mathrm{W}_{\hat{\rho} \pi} \mathrm{W}_{\hat{\rho} 4} \mathrm{~W}_{\hat{\pi} 4}=\left(\mathrm{W}_{\hat{\rho} \pi}\right)^{*} \mathrm{~W}_{1 \rho} \mathrm{W}_{\hat{\pi} 4} \mathrm{~W}_{\hat{\rho} \pi}
\end{aligned}
$$

where the first equality uses 2.12 , the second equality uses 2.13 and application of $\Sigma_{23}$, the third equality again uses 2.13 and the fourth equality uses 2.12.

Proof of Proposition 2.21, Let $(\pi, \hat{\pi})$ and $(\rho, \hat{\rho})$ be $\mathbb{G}$-Heisenberg and anti-Heisenberg pairs on $\mathcal{H}_{\pi}$ and $\mathcal{H}_{\rho}$ respectively. Lemma 2.22 forces $\pi \otimes \hat{\rho}$ and $\rho \otimes \hat{\pi}$ to be unitarily equivalent. By 10 , Proposition 5.3] the representations $\pi$ and $\rho$ of $A$ on $\mathcal{H}_{\pi}$ and $\mathcal{H}_{\rho}$ are quasi-equivalent. Similarly, $\hat{\rho}$ and $\hat{\pi}$ are quasi-equivalent representations of $\hat{A}$ on $\mathcal{H}_{\pi}$ and $\mathcal{H}_{\rho}$, respectively. Therefore, there is a unique quasi-equivalence class of representations of $A$ that contain the first element of all $\mathbb{G}$-Heisenberg and $\mathbb{G}$-anti-Heisenberg pairs. Similarly, there is a unique quasi-equivalence class of representations of $\hat{A}$ that contain the second element of all $\mathbb{G}$-Heisenberg and $\mathbb{G}$-anti-Heisenberg pairs. Moreover, Examples 2.18 and 2.19 show the existence of faithful $\mathbb{G}-H e i s e n b e r g$ and anti-Heisenberg pairs.

\footnotetext{
${ }^{1}$ Proposition 2.21 shows that the representations $\pi$ and $\hat{\pi}$ in a Heisenberg pair are always faithful.
} 
Example 2.23. Let $(\pi, \hat{\pi})$ be a $\mathbb{G}$-Heisenberg pair on $\mathcal{H}_{\pi}$ and let $\rho: A \rightarrow \mathbb{B}(\mathcal{K})$ be a faithful representation of $A$. Define $\pi^{\prime}(a):=(\rho \otimes \pi) \Delta_{A}(a)$ and $\hat{\pi}^{\prime}(\hat{a}):=1_{\mathcal{K}} \otimes \hat{\pi}(\hat{a})$ for $a \in A$ and $\hat{a} \in \hat{A}$. This is a $\mathbb{G}$-Heisenberg pair acting on $\mathcal{K} \otimes \mathcal{H}_{\pi}$.

Remark 2.24. $\widehat{\mathbb{G}}$-Heisenberg pairs are in one-to-one correspondence with $\mathbb{G}$-Heisenberg pairs: $(\pi, \hat{\pi})$ is a $\mathbb{G}$-Heisenberg pair on $\mathcal{H}_{\pi}$ if and only if $(\hat{\pi}, \pi)$ is a $\widehat{\mathbb{G}}$-Heisenberg pair on $\mathcal{H}_{\pi}$.

The pentagon equation 2.4 and Theorem 2.13(1) express the comultiplications using a $\mathbb{G}$-Heisenberg pair $(\pi, \hat{\pi})$ :

$$
\begin{aligned}
\Delta_{A}(a) & =\mathbb{W}\left(\pi(a) \otimes 1_{A}\right) \mathbb{W}^{*} & & \text { for all } a \in A, \\
\hat{\Delta}_{A}(\hat{a}) & =\sigma\left(\mathbb{W}^{*}\left(1_{\hat{A}} \otimes \hat{\pi}(\hat{a})\right) \mathbb{W}\right) & & \text { for all } \hat{a} \in \hat{A} .
\end{aligned}
$$

Warning 2.25. In (2.17), 2.18 we identify $a$ with $\pi(a)$ and $\hat{a}$ with $\hat{\pi}(\hat{a})$. We have used the same identification on the right side of Theorem 2.13|(6)|(ii)] We shall often follow this convention from now on.

Definition 2.26. Let $\mathcal{H}, \mathcal{K}$ be Hilbert spaces and let $\mathbb{V} \in \mathcal{U}(\mathcal{H} \otimes \mathcal{K})$. We call $\mathbb{V}$ good if, for any $a \in \mathbb{B}(\mathcal{H})$ and $b \in \mathbb{B}(\mathcal{K})$, we have

$$
\left(\mathbb{V}\left(a \otimes 1_{\mathcal{K}}\right) \mathbb{V}^{*}=1_{\mathcal{H}} \otimes b\right) \Rightarrow\left(a \in \mathbb{C} \cdot 1_{\mathcal{H}} \text { and } b \in \mathbb{C} \cdot 1_{\mathcal{K}}\right)
$$

The following Theorem is due to Woronowicz:

Theorem 2.27 ([28, Theorem 2.1]). Every modular multiplicative unitary is good.

Proof. Due to its importance, we prove this Theorem in detail. Define the operators $\hat{Q}, Q$, and $\widetilde{\mathbb{W}}$ as in Definition 2.10. First we prove the assertion without $D$ and under the additional assumption $b^{*} \mathcal{D} \operatorname{om}(Q) \subseteq \mathcal{D} \operatorname{Dom}(Q)$. Our assumption $\mathbb{W}(a \otimes 1)=(1 \otimes b) \mathbb{W}$ means

$$
(x \otimes y|\mathbb{W}| a z \otimes u)=\left(x \otimes b^{*} y|\mathbb{W}| z \otimes u\right)
$$

for all $x, z \in \mathcal{H}, y \in \mathcal{D} \operatorname{om}(Q)$ and $u \in \mathcal{D} \operatorname{om}\left(Q^{-1}\right)$. The modularity condition for $\mathbb{W}$ yields

$$
\left(\overline{a z} \otimes Q y|\widetilde{\mathbb{W}}| \bar{x} \otimes Q^{-1} u\right)=\left(\bar{z} \otimes Q b^{*} y|\widetilde{\mathbb{W}}| \bar{x} \otimes Q^{-1} u\right) .
$$

In this formula, $\widetilde{\mathbb{W}}\left(\bar{x} \otimes Q^{-1} u\right)$ runs through a dense subset of $\overline{\mathcal{H}} \otimes \mathcal{H}$. Since a vector in a Hilbert space is given by the linear functional it induces we get $\overline{a z} \otimes Q y=z \otimes Q b^{*} y$ for all $y \in \mathcal{D o m}(Q)$ and $z \in \mathcal{H}$. An operator $w$ on $\mathcal{H}$ induces a transpose operator $w^{\top}$ on $\overline{\mathcal{H}}$ by $w^{\top}(\bar{\xi}):=\overline{w^{*} \xi}$. Thus $\left(a^{*}\right)^{\top} \otimes 1=1 \otimes b^{*}$, so that $a, b \in \mathbb{C} \cdot 1$ and $a=b$.

To remove the assumption $b^{*} \mathcal{D} \operatorname{om}(Q) \subseteq \mathcal{D} \operatorname{om}(Q)$, we regularise $a$ and $b$. For $a \in \mathbb{B}(\mathcal{H})$ and $n \in \mathbb{N}$, we define

$$
\widehat{R}_{n}(a):=\int_{-\infty}^{+\infty} \widehat{Q}^{-\mathrm{i} t} a \widehat{Q}^{\mathrm{i} t} \delta_{n}(t) \mathrm{d} t \quad \text { and } \quad R_{n}(b):=\int_{-\infty}^{+\infty} Q^{-\mathrm{i} t} b Q^{\mathrm{i} t} \delta_{n}(t) \mathrm{d} t
$$

where

$$
\delta_{n}(t):=\sqrt{\frac{n}{2 \pi}} \exp \left(-\frac{n t^{2}}{2}\right)
$$

is a $\delta$-like sequence of Gaussian functions. Since

$$
(\mathbb{W})^{*}(\widehat{Q} \otimes Q) \mathbb{W}=\widehat{Q} \otimes Q,
$$

our condition $\mathbb{W}(a \otimes 1)=(1 \otimes b) \mathbb{W}$ implies

$$
\mathbb{W}\left(\widehat{R}_{n}(a) \otimes 1\right)=\left(1 \otimes R_{n}(b)\right) \mathbb{W} .
$$

We will show below that

$$
R_{n}(b)^{*} \mathcal{D} \operatorname{om}(Q) \subseteq \mathcal{D} \operatorname{om}(Q)
$$


The first part of the proof now yields $\widehat{R}_{n}(a)=R_{n}(b)=\lambda_{n} 1$ for all $n \in \mathbb{N}$. If $n \rightarrow \infty$, then $\widehat{R}_{n}(a)$ and $R_{n}(b)$ converge weakly towards $a$ and $b$, respectively. Hence we get $a=b=\lambda 1$ for some $\lambda \in \mathbb{C}$ in full generality.

It remains to establish 2.20 . Let $x, y \in \mathcal{D}$ om $(Q)$. Then the function

$$
f_{x, y}(z):=\left(Q^{\mathrm{i}(\bar{z}-\mathrm{i})} x\left|b^{*}\right| Q^{\mathrm{i} z} y\right)
$$

is well-defined, bounded, and continuous in the $\operatorname{strip} \Sigma:=\{z \in \mathbb{C}:-1 \leq \operatorname{Im} z \leq 0\}$ and holomorphic in the interior of $\Sigma$. In particular, for $t \in \mathbb{R}$ :

$$
f_{x, y}(t)=\left(Q x\left|Q^{-\mathrm{i} t} b^{*} Q^{\mathrm{i} t}\right| y\right), \quad f_{x, y}(t-\mathrm{i})=\left(x\left|Q^{-\mathrm{i} t} b^{*} Q^{\mathrm{i} t}\right| Q y\right) .
$$

By Cauchy's Theorem, the integrals of $f_{x, y}(z) \delta_{n}(z)$ along the lines $\mathbb{R}+$ is for $0 \leq 1 \leq s$ do not depend on $s$. For $s=0$ and $s=1,2.21$ shows that the integrals are $\left(Q x\left|R_{n}(b)^{*}\right| y\right)$ and

$$
\left(x\left|\int_{-\infty}^{+\infty} Q^{-\mathrm{i} t} b^{*} Q^{\mathrm{i} t} \delta_{n}(t-\mathrm{i}) \mathrm{d} t\right| Q y\right),
$$

respectively. Their equality shows that $\left(Q x \mid R_{n}(b)^{*} y\right)$ depends continuously on $x$. This yields $R_{n}(b)^{*} y \in$ $\operatorname{Dom}\left(Q^{*}\right)=\operatorname{Dom}(Q)$, that is, 2.20 .

Finally, we add the coefficient algebra $D$. If $a, b \in \mathcal{M}(\mathbb{K}(\mathcal{H}) \otimes D)$ satisfy $\mathbb{W}_{12} a_{13}=b_{23} \mathbb{W}_{12}$ in $\mathcal{M}(\mathbb{K}(\mathcal{H} \otimes \mathcal{H}) \otimes D)$, then the first part of the theorem applies to the slices (id $\otimes \mu)(a)$ and (id $\otimes \mu)(b)$ for all $\mu \in D^{\prime}$. Thus $(\mathrm{id} \otimes \mu)(a)=(\mathrm{id} \otimes \mu)(b)=\lambda_{\mu} \cdot 1$ for all $\mu \in D^{\prime}$. This implies that $a=b \in \mathbb{C} \cdot 1 \otimes \mathcal{M}(D)$.

The following result was proved in 24, Result 6.1] assuming the existence of Haar weights. We generalise it using Theorem 2.27 .

Lemma 2.28. Let $\mathbb{G}=\left(A, \Delta_{A}\right)$ be a quantum group constructed from a modular multiplicative unitary $\mathbb{W} \in$ $\mathbb{B}(\mathcal{H} \otimes \mathcal{H})$. If $a \in \mathcal{M}(A)$, then $\Delta_{A}(a) \in \mathcal{M}\left(A \otimes 1_{A}\right)$ or $\Delta_{A}(a) \in \mathcal{M}\left(1_{A} \otimes A\right)$ if and only if $a \in \mathbb{C} \cdot 1$.

More generally, if $D$ is a $\mathrm{C}^{*}$-algebra and $a \in \mathcal{M}(A \otimes D)$, then $\left(\Delta_{A} \otimes \operatorname{id}_{D}\right)(a) \in \mathcal{M}(A \otimes 1 \otimes D)$ or $\left(\Delta_{A} \otimes \operatorname{id}_{D}\right)(a) \in \mathcal{M}(1 \otimes A \otimes D)$ if and only if $a \in \mathbb{C} \cdot 1 \otimes \mathcal{M}(D)$.

Proof. Using (2.17), we rewrite the equation $\Delta_{A}(a)=1 \otimes a^{\prime}$ for $a, a^{\prime} \in \mathcal{M}(A \otimes D)$ as $\mathbb{W}_{12}^{A} a_{13}=a_{23}^{\prime} \mathbb{W}_{12}^{A}$. Now Theorem 2.27 yields $a \in \mathbb{C} \cdot 1 \otimes \mathcal{M}(D)$. If $\Delta_{A}(a)=a^{\prime} \otimes 1$ instead, then we apply the unitary antipodes. With $c:=\left(R_{A} \otimes \operatorname{id}_{D}\right)(a)$ and $c^{\prime}:=\left(R_{A} \otimes \operatorname{id}_{D}\right)\left(a^{\prime}\right)$, we get $\Delta_{A}(c)=1 \otimes c^{\prime}$. The argument above shows $c \in \mathbb{C} \cdot 1 \otimes \mathcal{M}(D)$ and hence $a \in \mathbb{C} \cdot 1 \otimes \mathcal{M}(D)$.

\subsection{Coactions of quantum groups}

Definition 2.29 (2, Définition 0.2]). A continuous (right) coaction of $\mathbb{G}$ on a $\mathrm{C}^{*}$-algebra $C$ is a morphism $\gamma: C \rightarrow C \otimes A$ with the following properties:

1. $\gamma$ is injective;

2. $\gamma$ is a comodule structure, that is, the following diagram commutes:

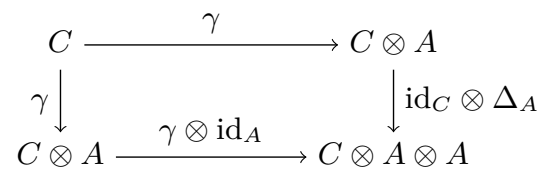

3. $\gamma$ satisfies the Podleś condition

$$
\gamma(C) \cdot\left(1_{C} \otimes A\right)=C \otimes A .
$$

We call $(C, \gamma)$ a $\mathbb{G}-\mathrm{C}^{*}$-algebra. We often drop $\gamma$ from our notation.

A morphism $f: C \rightarrow D$ between two $\mathbb{G}$-C $C^{*}$-algebras $\left(C, \gamma_{C}\right)$ and $\left(D, \gamma_{D}\right)$ is $\mathbb{G}$-equivariant if $\gamma_{D} \circ f=$ $\left(f \otimes \operatorname{id}_{A}\right) \circ \gamma_{C}$. Let $\operatorname{Mor}^{\mathbb{G}}(C, D)$ be the set of $\mathbb{G}$-equivariant morphisms from $C$ to $D$. 
Notation 2.30. Let $\mathfrak{C}^{*} \mathfrak{a l g}(\mathbb{G})$ be the category with $\mathbb{G}-\mathrm{C}^{*}$-algebras as objects and $\mathbb{G}$-equivariant morphisms as arrows.

Example 2.31. Any $\mathrm{C}^{*}$-algebra $C$ with the trivial coaction $\varphi^{\text {triv }}(c)=c \otimes 1_{A}$ for all $c \in C$ is an object of the category $\mathfrak{C}^{*} \mathfrak{a l g}(\mathbb{G})$.

Example 2.32. Theorem 2.13(3)|(i) implies that the underlying $\mathrm{C}^{*}$-algebra $A$ of a quantum group $\mathbb{G}=\left(A, \Delta_{A}\right)$ is a $\mathbb{G}-C^{*}$-algebra with $\Delta_{A}$ as the coaction.

Example 2.33. Combining the previous examples 2.31 and 2.32 we get $C \otimes A$ as an object in the category $\mathfrak{C}^{*} \mathfrak{a l g}(\mathbb{G})$ with the continuous $\mathbb{G}$-coaction $\varphi_{C \otimes A}:=\operatorname{id}_{C} \otimes \Delta_{A}$. The following lemma says that any continuous coaction may be embedded into one of this form.

Lemma 2.34. Let $C$ be a $\mathrm{C}^{*}$-algebra and $D$ a $\mathrm{C}^{*}$-subalgebra of $\mathcal{M}(C \otimes A)$ with

$$
\left(\operatorname{id}_{C} \otimes \Delta_{A}\right)(D) \cdot\left(1_{C \otimes A} \otimes A\right)=D \otimes A .
$$

Then $D$ with the coaction $\delta:=\left.\left(\operatorname{id}_{C} \otimes \Delta_{A}\right)\right|_{D}: D \rightarrow D \otimes A$ is a $\mathbb{G}-\mathrm{C}^{*}$-algebra, and the embedding $D \rightarrow$ $\mathcal{M}(C \otimes A)$ is a $\mathbb{G}$-equivariant morphism.

Every $\mathbb{G}-\mathrm{C}^{*}$-algebra is isomorphic to one of this form.

Proof. Equation (2.24) implies that $\operatorname{id}_{C} \otimes \Delta_{A}$ maps $D$ into $\mathcal{M}(D \otimes A)$ as claimed. Injectivity and coassociativity of $\delta$ are clear from 2.17) because they hold for $\operatorname{id}_{C} \otimes \Delta_{A}$, and 2.24] is the Podleś condition for $\delta$. Thus $\delta$ is a continuous $\mathbb{G}$-coaction. The equivariance of the embedding is clear.

Now let $(C, \gamma)$ be a $\mathbb{G}-C^{*}$-algebra. Let $D:=\gamma(C) \subseteq \mathcal{M}(C \otimes A)$. The comodule property 2.22 and the Podleś condition for $\gamma$ imply that $D$ satisfies 2.24):

$$
\begin{aligned}
\left(\operatorname{id}_{C} \otimes \Delta_{A}\right) \gamma(C) \cdot\left(1_{C \otimes A} \otimes A\right) & =\left(\gamma \otimes \operatorname{id}_{A}\right)\left(\gamma(C) \cdot\left(1_{C} \otimes A\right)\right) \\
& =\left(\gamma \otimes \operatorname{id}_{A}\right)(C \otimes A)=\gamma(C) \otimes A .
\end{aligned}
$$

Furthermore, the comodule property 2.22 for $\gamma$ implies that the isomorphism $\gamma: C \rightarrow D$ is $\mathbb{G}$-equivariant.

\subsection{Corepresentations of quantum groups}

Definition 2.35 ([2, Définition 0.3]). A (right) corepresentation of a $\mathrm{C}^{*}$-quantum group $\mathbb{G}=\left(A, \Delta_{A}\right)$ on a $\mathrm{C}^{*}$-algebra $D$ is a unitary $\mathrm{U} \in \mathcal{M}(D \otimes A)$ such that

$$
\left(\operatorname{id}_{D} \otimes \Delta_{A}\right) \mathrm{U}=\mathrm{U}_{12} \mathrm{U}_{13} \quad \text { in } \mathcal{U}(D \otimes A \otimes A) .
$$

In particular, if $D=\mathbb{K}(\mathcal{H})$ for some Hilbert space $\mathcal{H}$, we call $\mathrm{U}$ a corepresentation of $\mathbb{G}$ on $\mathcal{H}$.

Let $(\pi, \hat{\pi})$ be a $\mathbb{G}$-Heisenberg pair on a Hilbert space $\mathcal{H}$ such that $\mathbb{W}=(\hat{\pi} \otimes \pi) \mathrm{W} \in \mathcal{U}(\mathcal{H} \otimes \mathcal{H})$ be a manageable multiplicative unitary for $\mathbb{G}$. Let $\mathrm{U} \in \mathcal{U}(\mathbb{K}(\mathcal{K}) \otimes A)$ be a corepresentation of $\mathbb{G}$ on a Hilbert space $\mathcal{K}$ and let $\mathbb{U}:=\left(\operatorname{id}_{\mathcal{K}} \otimes \pi\right) \mathrm{U} \in \mathcal{U}(\mathcal{K} \otimes \mathcal{H})$. Using 2.17) we rewrite the corepresentation condition 2.25 in the following way

$$
\mathbb{W}_{23} \mathbb{U}_{12}=\mathbb{U}_{12} \mathbb{U}_{13} \mathbb{W}_{23} \quad \text { in } \mathcal{U}(\mathcal{K} \otimes \mathcal{H} \otimes \mathcal{H}) .
$$

Definition 1.3 in $[49$ shows that $\mathbb{U} \in \mathcal{U}(\mathcal{K} \otimes \mathcal{H})$ is adapted to $\mathbb{W}$. More generally, [49, Theorem 1.7] shows that every unitary adapted to $\mathbb{W}$ is a corepresentation of $\mathbb{G}$. Further, Theorem 1.6 in 49 ] shows that $\mathrm{U} \in \mathcal{U}(\mathbb{K}(\mathcal{K}) \otimes A)$ is manageable in the following sense: there is a unitary $\widetilde{\mathbb{U}} \in \mathcal{U}(\overline{\mathcal{K}} \otimes \mathcal{H})$ satisfying the following condition:

$$
(x \otimes u|\mathbb{U}| z \otimes y)=\left(\bar{z} \otimes Q u|\widetilde{\mathbb{U}}| \bar{x} \otimes Q^{-1} y\right),
$$

for all $x, z \in \mathcal{K}, u \in \mathcal{D} \operatorname{om}(Q)$ and $y \in \mathcal{D} \operatorname{om}\left(Q^{-1}\right)$. Here $Q$ is the positive, self-adjoint operator on $\mathcal{H}$ involved in the definition of the manageability of $\mathbb{W} \in \mathcal{U}(\mathcal{H} \otimes \mathcal{H})$.

The next Proposition shows that every corepresentation of $\mathbb{G}$ gives rise to a multiplicative unitary of $\mathbb{G}$.

Proposition 2.36. The unitary $\mathbb{W}_{13} \mathbb{U}_{23} \in \mathcal{U}(\mathcal{H} \otimes \mathcal{K} \otimes \mathcal{H} \otimes \mathcal{K})$ is a manageable multiplicative unitary on $\mathcal{H} \otimes \mathcal{K}$ generating $\mathbb{G}$. 
The following lemma is needed to show the manageability of $\mathbb{W}_{13} \mathbb{U}_{23}$.

Lemma 2.37. Let $Q$ be a self-adjoint, strictly positive operator acting on a Hilbert space $\mathcal{H}$. Then there is an orthonormal basis $\left\{e_{i}\right\}_{i=1,2, \cdots}$ in $\mathcal{H}$ such that all $e_{i} \in \mathcal{D} \operatorname{Dom}(Q) \cap \mathcal{D o m}\left(Q^{-1}\right)$ and

$$
\left.\sum_{i=1}^{\infty} \mid Q^{-1} e_{i}\right)\left(Q e_{i} \mid=1_{\mathcal{H}}\right.
$$

where the sum converges in the strong topology.

Proof. Given any integer $n \in \mathbb{Z}$ and any $\lambda \in\left[2^{2 n-1}, 2^{2 n+1}\right)$ we set $f(\lambda)=2^{-2 n} \lambda$. Then

$$
\mathbb{R}_{>0} \ni \lambda \mapsto f(\lambda) \in\left[2^{-1}, 2\right)
$$

is a piecewise linear function and the operator $Q^{\prime}:=f(Q)$ is self-adjoint, bounded with bounded inverse: $\left\|Q^{\prime}\right\|,\left\|Q^{\prime-1}\right\| \leq 2$. Let $\chi$ denote the characteristic function (not bicharacter) as discussed in 52 such that $\chi\left(2^{2 n-1} \leq Q \leq 2^{2 n+1}\right)$ is the spectral projection assigned to $\operatorname{Sp}(Q) \cap\left[2^{2 n-1}, 2^{2 n+1}\right)$. Moreover, if $e \in \mathcal{H}$ and

$$
\chi\left(2^{2 n-1} \leq Q<2^{2 n+1}\right) e=e
$$

then $e \in \operatorname{Dom}(Q) \cap \mathcal{D o m}\left(Q^{-1}\right), Q e=2^{2 n} f(Q)(e)=2^{2 n} Q^{\prime} e$ and $Q^{-1} e=2^{-2 n} f\left(Q^{-1}\right) e=2^{-2 n} Q^{\prime-1}$. In that case

$$
\left.\mid Q^{-1} e\right)\left(Q e|=| Q^{\prime-1} e\right)\left(Q^{\prime} e\left|=Q^{\prime-1}\right| e\right)\left(e \mid Q^{\prime} .\right.
$$

Finally, selecting an orthonormal basis $\left\{e_{i}\right\}_{i=1,2, \ldots}$ in $\mathcal{H}$ with all $e_{i}$ satisfying condition 2.29 ( $n$ depends on $i$ ) we get

$$
\left.\sum_{i=1}^{\infty} \mid Q^{-1} e_{i}\right)\left(Q e_{i}\left|=\sum_{i=1}^{\infty} Q^{\prime-1}\right| e_{i}\right)\left(e_{i} \mid Q^{\prime}=Q^{\prime-1}\left(\sum_{i=1}^{\infty} \mid e_{i}\right)\left(e_{i} \mid\right) Q^{\prime}=1_{\mathcal{H}}\right.
$$

Proof of Proposition 2.36. Let $X_{1234}:=\mathbb{W}_{13} \mathbb{U}_{23}$. The following computation shows that $X_{1234}$ satisfies the pentagon equation (2.4):

$$
\begin{aligned}
X_{3456} X_{1234} X_{3456}=\mathbb{W}_{35} \mathbb{U}_{45} \mathbb{W}_{13} \mathbb{U}_{23} \mathbb{U}_{45}^{*} \mathbb{W}_{35}^{*}=\mathbb{W}_{35} \mathbb{W}_{13} \mathbb{U}_{23} \mathbb{W}_{35}^{*}=\mathbb{W}_{13} \mathbb{W}_{15} \mathbb{W}_{35} \mathbb{U}_{23} \mathbb{W}_{35}^{*} & =\mathbb{W}_{13} \mathbb{W}_{15} \mathbb{U}_{23} \mathbb{U}_{25} \\
& =X_{1234} X_{1256}
\end{aligned}
$$

The first equality follows from the assumption, the second equality holds as $\mathbb{U}_{45}$ and $\mathbb{W}_{13} \mathbb{U}_{23}$ commute, the third equality uses (2.4), the fourth equality follows from 2.26) and the fifth equality is trivial.

Clearly, $X_{1234} \in \mathcal{U}(\hat{A} \otimes \mathbb{K}(\mathcal{K}) \otimes A \otimes 1)$. We show that the $\mathrm{C}^{*}$-algebra generated by slices on the first two legs of $X_{1234}$ is indeed $A$. Manageability of $\mathbb{W} \in \mathcal{U}(\mathcal{H} \otimes \mathcal{H})$ yields $A=\left\{(\omega \otimes\right.$ id $\left.) \mathbb{W}^{*}: \omega \in \mathbb{B}(\mathcal{H})_{*}\right\}$. We replace $\mathbb{B}(\mathcal{K})_{*}$ by the dense subset $\xi \omega^{\prime}$, where $\xi \omega^{\prime}$ is defined by $\xi \omega^{\prime}\left(\xi^{\prime}\right):=\omega^{\prime}\left(\xi^{\prime} \cdot \xi\right)$ for $\xi, \xi^{\prime} \in \mathbb{B}(\mathcal{K})$ and $\omega^{\prime} \in \mathbb{B}(\mathcal{K})_{*}$. Therefore,

$$
\begin{aligned}
& \left\{\left(\omega \otimes \xi \omega^{\prime} \otimes \mathrm{id} \otimes \mathrm{id}_{\mathcal{K}}\right) X_{1234}^{*}: \omega \in \mathbb{B}(\mathcal{H})_{*}, \omega^{\prime} \in \mathbb{B}(\mathcal{K})_{*}, \xi \in \mathbb{B}(\mathcal{K})\right\}^{\mathrm{CLS}} \\
& =\left\{\left(\xi \cdot \omega^{\prime} \otimes \mathrm{id}\right) \mathbb{U}^{*} \cdot a: a \in A, \omega^{\prime} \in \mathbb{B}(\mathcal{K})_{*}, \xi \in \mathbb{B}(\mathcal{K})\right\}^{\mathrm{CLS}} \\
& =\left\{\left(\omega^{\prime} \otimes \mathrm{id}\right)\left(\mathbb{U}^{*}(\xi \otimes a)\right): a \in A, \omega^{\prime} \in \mathbb{B}(\mathcal{K})_{*}, \xi \in \mathbb{B}(\mathcal{K})\right\}^{\mathrm{CLS}} \\
& =\left\{\left(\omega^{\prime} \otimes \mathrm{id}\right)(\xi \otimes a): a \in A, \omega^{\prime} \in \mathbb{B}(\mathcal{K})_{*}, \xi \in \mathbb{B}(\mathcal{K})\right\}^{\mathrm{CLS}}=A
\end{aligned}
$$

Finally we show that $X_{1234}$ is manageable. Let $Q$ be the positive, self-adjoint operator on $\mathcal{H}$ involved in the definition of the manageability of $\mathbb{W} \in \mathcal{U}(\mathcal{H} \otimes \mathcal{H})$. Define $\mathbb{Q}:=Q \otimes 1_{\mathcal{K}}$. Clearly, $\mathbb{Q} \otimes \mathbb{Q}$ commutes with $X_{1234}$. 
By Lemma 2.37 there is an orthonormal basis $\left\{e_{i}\right\}_{i=1,2 \ldots}$ of $\mathcal{H}$ such that $e_{i} \in \mathcal{D} \operatorname{Dom}(Q) \cap \mathcal{D o m}\left(Q^{-1}\right)$ satisfying 2.28). Let $x, z \in \mathcal{H}, x_{1}, z_{1} \in \mathcal{K}, u \in \mathcal{D o m}(Q)$ and $y \in \mathcal{D o m}\left(Q^{-1}\right)$. We compute

$$
\begin{aligned}
& \left(x_{1} \otimes x_{2} \otimes u\left|\mathbb{W}_{13} \mathbb{U}_{23}\right| z_{1} \otimes z_{2} \otimes y\right) \\
& =\sum_{i}\left(x_{1} \otimes x_{2} \otimes u\left|\mathbb{W}_{13} \cdot\left(1 \otimes 1 \otimes \mid e_{i}\right) \cdot\left(e_{i} \mid\right) \cdot \mathbb{U}_{23}\right| z_{1} \otimes z_{2} \otimes y\right) \\
& =\sum_{i}\left(x_{1} \otimes u|\mathbb{W}| z_{1} \otimes e_{i}\right) \cdot\left(x_{2} \otimes e_{i}|\mathbb{U}| z_{2} \otimes y\right) \\
& =\sum_{i}\left(\overline{z_{1}} \otimes Q u|\widetilde{\mathbb{W}}| \overline{x_{1}} \otimes Q^{-1} e_{i}\right) \cdot\left(\overline{z_{2}} \otimes Q e_{i}|\widetilde{\mathbb{U}}| \overline{x_{2}} \otimes Q^{-1} y\right) \\
& =\sum_{i}\left(\overline{z_{1}} \otimes \overline{z_{2}} \otimes Q u\left|\widetilde{\mathbb{W}}_{13} \cdot\left(1 \otimes 1 \otimes \mid Q^{-1} e_{i}\right) \cdot\left(Q e_{i} \mid\right) \cdot \widetilde{\mathbb{U}}_{23}\right| \overline{x_{1}} \otimes \overline{x_{2}} \otimes Q^{-1} y\right) \\
& =\left(\overline{z_{1}} \otimes \overline{z_{2}} \otimes Q u\left|\widetilde{\mathbb{W}}_{13} \widetilde{\mathbb{U}}_{23}\right| \overline{x_{1}} \otimes \overline{x_{2}} \otimes Q^{-1} y\right) .
\end{aligned}
$$

Hence, $X_{1234} \in \mathcal{U}(\mathcal{H} \otimes \mathcal{K} \otimes \mathcal{H} \otimes \mathcal{K})$ is a manageable multiplicative unitary generating $\mathbb{G}$.

Proposition 2.38. Let $\mathrm{U} \in \mathcal{U}\left(D_{1} \otimes A\right)$ and $\mathrm{V} \in \mathcal{U}\left(D_{2} \otimes \hat{A}\right)$ be corepresentations of $\mathbb{G}$, $\widehat{\mathbb{G}}$ on $\mathrm{C}^{*}$-algebras $D_{1}$, $D_{2}$. Then there is a unique unitary $Z \in \mathcal{U}\left(D_{1} \otimes D_{2}\right)$ such that for any $\mathbb{G}$-Heisenberg pair $(\pi, \hat{\pi})$ on $\mathcal{L}$

$$
\mathrm{U}_{1 \pi} \mathrm{V}_{2 \hat{\pi}} Z_{12}=\mathrm{V}_{2 \hat{\pi}} \mathrm{U}_{1 \pi} \quad \text { in } \mathcal{U}\left(D_{1} \otimes D_{2} \otimes \mathbb{K}(\mathcal{L})\right) .
$$

Equivalently, $Z \in \mathcal{U}\left(D_{1} \otimes D_{2}\right)$ satisfies the following:

$$
\hat{\mathrm{V}}_{\hat{\pi} 3} \mathrm{U}_{1 \pi}=\mathrm{U}_{1 \pi} Z_{13}^{*} \hat{\mathrm{V}}_{\hat{\pi} 3} \quad \text { in } \mathcal{U}\left(D_{1} \otimes \mathbb{K}(\mathcal{L}) \otimes D_{2}\right),
$$

where $\hat{\mathrm{V}}=\sigma\left(\mathrm{V}^{*}\right) \in \mathcal{U}\left(\hat{A} \otimes \mathbb{K} D_{2}\right)$.

In particular, if $D_{1}=\mathbb{K}(\mathcal{H})$ and $D_{2}=\mathbb{K}(\mathcal{K})$ for some Hilbert spaces $\mathcal{H}$ and $\mathcal{K}$, then $Z \in \mathcal{U}(\mathcal{H} \otimes \mathcal{K})$.

Proof. Let $\mathrm{W} \in \mathcal{U}(\hat{A} \otimes A)$ be the reduced bicharacter of $\mathbb{G}$. Equations 2.17) and 2.25) for U yield:

$$
\mathrm{W}_{\hat{\pi} 3} \mathrm{U}_{1 \pi}=\mathrm{U}_{1 \pi} \mathrm{U}_{13} \mathrm{~W}_{\hat{\pi} 3} \quad \text { in } \mathcal{U}\left(D_{1} \otimes \mathbb{K}(\mathcal{L}) \otimes A\right) .
$$

Similarly, equations 2.18 and 2.25 for $\hat{V}$ gives:

$$
\mathrm{V}_{13} \mathrm{~W}_{\hat{\pi} 3}=\mathrm{W}_{\hat{\pi} 3} \mathrm{~V}_{13} \mathrm{~V}_{1 \pi} \quad \text { in } \mathcal{U}\left(D_{2} \otimes \mathbb{K}(\mathcal{L}) \otimes \hat{A}\right) .
$$

Define $\widetilde{Z}:=\mathrm{V}_{2 \pi}^{*} \mathrm{U}_{1 \pi}^{*} \mathrm{~V}_{2 \hat{\pi}} \mathrm{U}_{1 \pi} \in \mathcal{U}\left(D_{1} \otimes D_{2} \otimes \mathbb{K}(\mathcal{L})\right)$ and consider the multiplicative unitary $\mathbb{W}=$ $(\hat{\pi} \otimes \pi) \mathrm{W} \in \mathcal{U}(\mathcal{L} \otimes \mathcal{L})$. We are going to use Theorem 2.27 to show $\widetilde{Z} \in \mathcal{U}\left(D_{1} \otimes D_{1} \otimes 1\right)$.

$$
\begin{aligned}
\mathbb{W}_{34} \mathrm{~V}_{23}^{*} \mathrm{U}_{13}^{*} \mathrm{~V}_{23} \mathrm{U}_{13}\left(\mathbb{W}_{34}\right)^{*}=\mathrm{V}_{24}^{*} \mathbb{W}_{34} \mathrm{~V}_{24} \mathrm{U}_{13}^{*} \mathrm{~V}_{23}\left(\mathbb{W}_{34}\right)^{*} \mathrm{U}_{13} \mathrm{U}_{14} & =\mathrm{V}_{24}^{*} \mathbb{W}_{34} \mathrm{U}_{13}^{*} \mathrm{~V}_{24} \mathrm{~V}_{23}\left(\mathbb{W}_{34}\right)^{*} \mathrm{U}_{13} \mathrm{U}_{14} \\
& =\mathrm{V}_{24}^{*} \mathrm{U}_{14}^{*} \mathrm{U}_{13}^{*} \mathrm{~V}_{24} \mathrm{U}_{13} \mathrm{U}_{14} \\
& =\mathrm{V}_{24}^{*} \mathrm{U}_{14}^{*} \mathrm{~V}_{24} \mathrm{U}_{14}
\end{aligned}
$$

where the first and third equality use 2.32 and 2.33), whereas the second and fourth equality follow because $\mathrm{U}_{13}$ and $\mathrm{V}_{24}$ commute.

Now Theorem 2.27 gives a unitary $Z \in \mathcal{U}\left(D_{1} \otimes D_{2}\right)$ such that $Z_{12}=\widetilde{Z}$. It satisfies 2.30), which also shows the uniqueness.

Remark 2.39. At first sight, it seems that the unitary $Z \in \mathcal{U}(\mathcal{H} \otimes \mathcal{K})$ in Proposition 2.38 depends on the choice of $\mathbb{G}$-Heisenberg pairs. We shall justify its independence of auxiliary choices of $\mathbb{G}$-Heisenberg pairs in Remark 2.44. 


\subsection{Universal duals of quantum groups}

Given a locally compact group $G$, the full group $\mathrm{C}^{*}$-algebra $\mathrm{C}^{*}(G)$ is universal in the following sense: every unitary representation of $G$ on a Hilbert space factors through a representation of $\mathrm{C}^{*}(G)$ on the same Hilbert space. The comultiplication $\Delta_{\mathrm{C}^{*}(G)} \in \operatorname{Mor}\left(\mathrm{C}^{*}(G), \mathrm{C}^{*}(G) \otimes \mathrm{C}^{*}(G)\right)$ defined by $\Delta_{\mathrm{C}^{*}(G)}(a):=a \otimes a$ for $a=\delta_{g}$ is coassociative. Furthermore, $\left(\mathrm{C}^{*}(G), \Delta_{\mathrm{C}^{*}(G)}\right)$ is a bisimplifiable Hopf $\mathrm{C}^{*}$-algebra and $\left(\mathrm{C}_{\mathrm{r}}^{*}(G), \Delta_{\mathrm{C}_{\mathrm{r}}^{*}(G)}\right)$ is a quotient of it. Thus $\mathrm{C}^{*}(G)$ can be considered as the universal dual of the quantum group $\mathrm{C}_{0}(G)$. The counterpart for general quantum groups is known as the universal dual of the quantum group. Under the assumption of Haar weights, Kustermans discussed a general theory of it in 22. Later, in 39], Soltan and Woronowicz generalised Kustermans's result in the framework of modular multiplicative unitaries. Let us recall some facts from 39 in the following theorem:

Theorem 2.40. Let $\mathbb{G}=\left(A, \Delta_{A}\right)$ be a quantum group.

1. There is a bisimplifiable Hopf $\mathrm{C}^{*}$-algebra $\widehat{\mathbb{G}}^{\mathrm{u}}=\left(\hat{A}^{\mathrm{u}}, \hat{\Delta}_{A^{\mathrm{u}}}\right)$.

2. There is a unique corepresentation $\tilde{\mathcal{V}} \in \mathcal{U}\left(\hat{A}^{\mathrm{u}} \otimes A\right)$ of $\mathbb{G}$ such that

(a) $\tilde{\mathcal{V}}$ is universal: given any corepresentation $U \in \mathcal{U}(D \otimes A)$ of $\mathbb{G}$ on a $\mathrm{C}^{*}$-algebra $D$ there is a unique $\varphi \in \operatorname{Mor}\left(\hat{A}^{\mathrm{u}}, D\right)$ with

$$
\left(\varphi \otimes \operatorname{id}_{A}\right) \tilde{\mathcal{V}}=U
$$

(b) $\tilde{\mathcal{V}}$ is a character in the first leg:

$$
\left(\hat{\Delta}_{A}^{\mathrm{u}} \otimes \mathrm{id}_{A}\right) \tilde{\mathcal{V}}=\tilde{\mathcal{V}}_{23} \tilde{\mathcal{V}}_{13}
$$

(c) slices on the first leg of $\tilde{\mathcal{V}}$ generate the $\mathrm{C}^{*}$-algebra $\hat{A}^{\mathrm{u}}$ :

$$
\hat{A}^{\mathrm{u}}=\left\{\left(\operatorname{id}_{\hat{A}^{\mathrm{u}}} \otimes \omega\right) \tilde{\mathcal{V}} \mid \omega \in A^{\prime}\right\}^{\mathrm{CLS}}
$$

As a consequence of Theorem 2.40,2) there is a unique Hopf *-homomorphism (see 39 . Proposition 34]) $\hat{\Lambda}: \hat{A}^{\mathrm{u}} \rightarrow \hat{A}$, known as the (dual) reducing morphism, satisfying

$$
\left(\hat{\Lambda} \otimes \operatorname{id}_{A}\right)(\tilde{\mathcal{V}})=\mathrm{W}
$$

Also, there is a unique bounded dual counit $\hat{e}^{\mathrm{u}}: \hat{A}^{\mathrm{u}} \rightarrow \mathbb{C}$ (see 39 , Proposition 31]) characterised by the trivial corepresentation of $\mathbb{G}$ :

$$
\left(\hat{e}^{\mathrm{u}} \otimes \operatorname{id}_{A}\right) \tilde{\mathcal{V}}=1_{A},
$$

with the following property:

$$
\left(\hat{e}^{\mathrm{u}} \otimes \mathrm{id}_{\hat{A}^{\mathrm{u}}}\right) \hat{\Delta}_{A}^{\mathrm{u}}=\left(\operatorname{id}_{\hat{A}^{\mathrm{u}}} \otimes \hat{e}^{\mathrm{u}}\right) \hat{\Delta}_{A}^{\mathrm{u}}=\mathrm{id}_{\hat{A}^{\mathrm{u}}} .
$$

Similarly $\mathbb{G}^{\mathrm{u}}=\left(A^{\mathrm{u}}, \Delta_{A^{\mathrm{u}}}\right)$ is the universal dual of $\widehat{\mathbb{G}}$ with a unique corepresentation $\mathcal{V} \in \mathcal{U}\left(\hat{A} \otimes A^{\mathrm{u}}\right)$ of $\widehat{\mathbb{G}}$ such that

1. $\mathcal{V}$ is universal: given any corepresentation $U \in \mathcal{U}(\hat{A} \otimes D)$ of $\widehat{\mathbb{G}}$ on a $\mathrm{C}^{*}$-algebra $D$ there is a unique $\varphi \in \operatorname{Mor}\left(A^{\mathrm{u}}, D\right)$ satisfying

$$
\left(\operatorname{id}_{\hat{A}} \otimes \varphi\right) \mathcal{V}=U
$$

2. $\mathcal{V}$ is a character in the second leg:

$$
\left(\operatorname{id}_{\hat{A}} \otimes \Delta_{A}^{\mathrm{u}}\right) \mathcal{V}=\mathcal{V}_{12} \mathcal{V}_{13}
$$

3. slices on the second leg of $\mathcal{V}$ generate the $\mathrm{C}^{*}$-algebra $A^{\mathrm{u}}$ :

$$
A^{\mathrm{u}}=\left\{\left(\omega \otimes \mathrm{id}_{A^{\mathrm{u}}}\right) \mathcal{V} \mid \omega \in \hat{A}^{\prime}\right\}^{\|\cdot\| \text {-closure }}
$$


The unique Hopf ${ }^{*}$-homomorphism, reducing morphism, $\Lambda \in \operatorname{Mor}\left(A^{\mathrm{u}}, A\right)$ is characterised by the following condition:

$$
\left(\operatorname{id}_{\hat{A}} \otimes \Lambda\right)(\mathcal{V})=\mathrm{W}
$$

Finally, we also have a unique bounded counit $e^{\mathrm{u}}: A^{\mathrm{u}} \rightarrow \mathbb{C}$ characterised by:

$$
\left(\operatorname{id}_{\hat{A}} \otimes e^{\mathrm{u}}\right) \mathcal{V}=1_{\hat{A}} \text {. }
$$

It satisfies

$$
\left(e^{\mathrm{u}} \otimes \mathrm{id}_{A^{\mathrm{u}}}\right) \Delta_{A}^{\mathrm{u}}=\left(\mathrm{id}_{A^{\mathrm{u}}} \otimes e^{\mathrm{u}}\right) \Delta_{A}^{\mathrm{u}}=\mathrm{id}_{A^{\mathrm{u}}} .
$$

The following result due to Kustermans which works in the setting of modular multiplicative unitaries:

Lemma 2.41 (22, Result 6.1]). Let $X, Y \in \mathcal{U}\left(D \otimes A^{\mathrm{u}}\right)$ be corepresentations of $\mathbb{G}^{\mathrm{u}}$ on a $\mathrm{C}^{*}$-algebra D. Let $\Lambda_{A}: A^{\mathrm{u}} \rightarrow A$ be the reducing morphism. If $\left(\mathrm{id}_{D} \otimes \Lambda_{A}\right) X=\left(\operatorname{id}_{D} \otimes \Lambda_{A}\right) Y$, then $X=Y$. A similar statement holds in the first variable.

Universal duals of quantum groups, in general, do not arise from modular or manageable multiplicative unitaries; hence they are not quantum groups. Still one may lift $\mathrm{W} \in \mathcal{U}(\hat{A} \otimes A)$ uniquely to a unitary $\mathcal{W} \in$ $\mathcal{U}\left(\hat{A}^{\mathrm{u}} \otimes A^{\mathrm{u}}\right)$ satisfying a variant of pentagon equation 2.4 :

Proposition 2.42. There is a unique unitary $\mathcal{W} \in \mathcal{U}\left(\hat{A}^{\mathrm{u}} \otimes A^{\mathrm{u}}\right)$ such that

$$
\begin{aligned}
\left(\operatorname{id}_{\hat{A}^{\mathrm{u}}} \otimes \Delta_{A^{\mathrm{u}}}\right) \mathcal{W} & =\mathcal{W}_{12} \mathcal{W}_{13} & & \text { in } \mathcal{U}\left(\hat{A}^{\mathrm{u}} \otimes A^{\mathrm{u}} \otimes A^{\mathrm{u}}\right), \\
\left(\hat{\Delta}_{A^{\mathrm{u}}} \otimes \mathrm{id}_{\hat{A}^{\mathrm{u}}}\right) \mathcal{W} & =\mathcal{W}_{23} \mathcal{W}_{13} & & \text { in } \mathcal{U}\left(\hat{A}^{\mathrm{u}} \otimes \hat{A}^{\mathrm{u}} \otimes A^{\mathrm{u}}\right), \\
\left(\operatorname{id}_{\hat{A}^{\mathrm{u}}} \otimes \Lambda\right) \mathcal{W} & =\tilde{\mathcal{V}} & & \text { in } \mathcal{U}\left(\hat{A}^{\mathrm{u}} \otimes A\right), \\
\left(\hat{\Lambda} \otimes \mathrm{id}_{A^{\mathrm{u}}}\right) \mathcal{W} & =\mathcal{V} & & \text { in } \mathcal{U}\left(\hat{A} \otimes A^{\mathrm{u}}\right), \\
(\hat{\Lambda} \otimes \Lambda) \mathcal{W} & =\mathrm{W} & & \text { in } \mathcal{U}(\hat{A} \otimes A), \\
\left(\operatorname{id}_{\hat{A}^{\mathrm{u}}} \otimes e^{\mathrm{u}}\right) \mathcal{W} & =1_{\hat{A}^{\mathrm{u}}} & & \text { in } \mathcal{U}\left(\hat{A}^{\mathrm{u}}\right), \\
\left(\hat{e}^{\mathrm{u}} \otimes \mathrm{id}_{A^{\mathrm{u}}}\right) \mathcal{W} & =1_{A^{\mathrm{u}}} & & \text { in } \mathcal{U}\left(A^{\mathrm{u}}\right) .
\end{aligned}
$$

Definition 2.43. The unitary multiplier $\mathcal{W}$ in Proposition 2.42 is called the universal bicharacter of $\mathbb{G}=\left(A, \Delta_{A}\right)$.

In the presence of a Haar weight on quantum groups this was shown by Kustermans 22, Proposition 6.4]. We establish this fact in the context of modular multiplicative unitaries.

Proof of Proposition 2.42, Let $(\pi, \hat{\pi})$ be a $\mathbb{G}$-Heisenberg pair. Proposition 2.38 gives a unique $\mathcal{W} \in$ $\mathcal{U}\left(\hat{A}^{\mathrm{u}} \otimes A^{\mathrm{u}}\right)$ with

Now we show 2.46:

$$
\mathcal{W}_{13}=\tilde{\mathcal{V}}_{1 \pi}^{*} \mathcal{V}_{\hat{\pi} 3} \tilde{\mathcal{V}}_{1 \pi} \mathcal{V}_{\hat{\pi} 3}^{*}
$$

$$
\begin{aligned}
& \left(\operatorname{id}_{\hat{A}^{\mathrm{u}}} \otimes \operatorname{id}_{A} \otimes \Delta_{A^{\mathrm{u}}}\right) \tilde{\mathcal{V}}_{1 \pi}^{*} \mathcal{V}_{\hat{\pi} 3} \tilde{\mathcal{V}}_{1 \pi} \mathcal{V}_{\hat{\pi} 3}^{*}=\tilde{\mathcal{V}}_{1 \pi}^{*} \mathcal{V}_{\hat{\pi} 3} \mathcal{V}_{\hat{\pi} 4} \tilde{\mathcal{V}}_{1 \pi} \mathcal{V}_{\hat{\pi} 4}^{*} \mathcal{V}_{\hat{\pi} 3}^{*}=\mathcal{W}_{13} \mathcal{V}_{\hat{\pi} 3} \tilde{\mathcal{V}}_{1 \pi}^{*} \mathcal{V}_{\hat{\pi} 4} \tilde{\mathcal{V}}_{1 \pi} \mathcal{V}_{\hat{\pi} 4}^{*} \mathcal{V}_{\hat{\pi} 3}^{*} \\
& =\mathcal{W}_{13} \mathcal{V}_{\hat{\pi} 3} \mathcal{W}_{14} \mathcal{V}_{\hat{\pi} 3}^{*}=\mathcal{W}_{13} \mathcal{W}_{14}
\end{aligned}
$$

A similar routine computation yields 2.47). The following computation yields 2.48):

$$
\left(\operatorname{id}_{\hat{A}^{\mathrm{u}}} \otimes \operatorname{id}_{A} \otimes \Lambda\right) \mathcal{W}_{13}=\left(\operatorname{id}_{\hat{A}^{\mathrm{u}}} \otimes \mathrm{id}_{A} \otimes \Lambda\right) \tilde{\mathcal{V}}_{12}^{*} \mathcal{V}_{23} \tilde{\mathcal{V}}_{12} \mathcal{V}_{23}^{*}=\tilde{\mathcal{V}}_{12}^{*} \mathrm{~W}_{23} \tilde{\mathcal{V}}_{12} \mathrm{~W}_{23}^{*}=\tilde{\mathcal{V}}_{12}^{*} \tilde{\mathcal{V}}_{12} \tilde{\mathcal{V}}_{13}=\tilde{\mathcal{V}}_{13}
$$

A similar computation yields 2.49). Then 2.50 follows from 2.43 or 2.37). Finally, 2.51) and 2.52 follow by using 2.44) and 2.38$)$ in 2.53 .

Let $\left(\pi^{\prime}, \hat{\pi}^{\prime}\right)$ be another $\mathbb{G}-$ Heisenberg pair and $\mathcal{W}^{\prime} \in \mathcal{U}\left(\hat{A}^{\mathrm{u}} \otimes A^{\mathrm{u}}\right)$ be the corresponding universal bicharacter. Now 2.48 gives $\left(\operatorname{id}_{\hat{A}^{\mathrm{u}}} \otimes \Lambda\right) \mathcal{W}=\left(\operatorname{id}_{\hat{A}^{\mathrm{u}}} \otimes \Lambda\right) \mathcal{W}^{\prime}=\mathcal{V}$; Lemma 2.41 gives $\mathcal{W}=\mathcal{W}^{\prime}$.

Remark 2.44. We conclude by arguing that $Z \in \mathcal{U}(\mathcal{H} \otimes \mathcal{K})$ in Proposition 2.38 satisfying 2.31), is independent of the choice of $\mathbb{G}$-Heisenberg pairs. The universal property 2.34$)$ of $\mathcal{V} \in \mathcal{U}\left(\hat{A}^{\mathrm{u}} \otimes A\right)$ gives a unique $\varphi \in \operatorname{Mor}\left(\hat{A}^{\mathrm{u}}, D_{1}\right)$ with $\left(\varphi \otimes \mathrm{id}_{A}\right) \tilde{\mathcal{V}}=\mathrm{U}$. Similarly, by the universal property 2.40$)$ of $\mathcal{V} \in \mathcal{U}\left(\hat{A} \otimes A^{\mathrm{u}}\right)$, there is a unique $\psi \in \operatorname{Mor}\left(A^{\mathrm{u}}, D_{2}\right)$ such that $\left(\operatorname{id}_{\hat{A}} \otimes \psi\right) \mathcal{V}=\hat{\mathrm{V}}$. Let $(\pi, \hat{\pi})$ be a $\mathbb{G}$-Heisenberg pair acting on $\mathcal{L}$ and define $Z:=(\varphi \otimes \psi) \mathcal{W}^{*} \in \mathcal{U}\left(D_{1} \otimes D_{2}\right)$. Since $\mathcal{W} \in \mathcal{U}\left(\hat{A}^{\mathrm{u}} \otimes A^{\mathrm{u}}\right)$ is independent of the choice of $\mathbb{G}$-Heisenberg pairs so is $Z$. Finally, applying $\varphi \otimes \mathrm{id}_{\mathcal{L}} \otimes \psi$ on both sides of 2.53) we obtain 2.30. 


\section{Chapter 3}

\section{Homomorphisms of quantum groups}

Let $G$ and $H$ be two locally compact groups and let $\hat{G}$ and $\hat{H}$ be the respective Pontrjagin duals. Then for any continuous group homomorphism $f: G \rightarrow H$ there is a unique dual morphism $\hat{f}: \hat{H} \rightarrow \hat{G}$ defined as $\hat{f}\left(\chi_{h}\right):=\chi_{f(h)}$, where $\chi_{h} \in \hat{H}$ for all $h \in H$. There should be a similar notion of homomorphisms of quantum groups which is compatible with the duality in the following sense: given a quantum group homomorphism $f: \mathbb{G} \rightarrow \mathbb{H}$ there is a unique quantum group homomorphism $\hat{f}: \widehat{\mathbb{H}} \rightarrow \widehat{\mathbb{G}}$. A natural choice would be the Hopf *-homomorphisms in Definition 2.4, which are the right morphisms between compact quantum groups (see [43]) and, more generally, between amenable quantum groups. For locally compact groups, however, they are not appropriate because they do not behave well for reduced group $\mathrm{C}^{*}$-algebras. It is easy to see that a group homomorphism $f: G \rightarrow H$ induces Hopf *-homomorphisms from $\mathrm{C}_{0}(H)$ to $\mathrm{C}_{0}(G)$ and from $\mathrm{C}^{*}(G)$ to $\mathrm{C}^{*}(H)$. But the latter does not always descend to the reduced group $\mathrm{C}^{*}$-algebras. For instance, the constant map from $G$ to the trivial group $\{1\}$ induces a Hopf ${ }^{*}$-homomorphism $\mathrm{C}_{\mathrm{r}}^{*}(G) \rightarrow \mathrm{C}_{\mathrm{r}}^{*}(\{1\})=\mathbb{C}$ if and only if $G$ is amenable. In this chapter we propose equivalent notions of homomorphisms of quantum groups building on work by $\mathrm{Ng} 31$ and later by Kustermans 22 .

In Section 3.1 we recall the theory of bicharacters as quantum group homomorphisms, which is nicely compatible with the duality of quantum groups. In Section 3.2 we show a bijection between bicharacters and Hopf *-homomorphisms of universal quantum group $\mathrm{C}^{*}$-algebras. In Section 3.3 we interpret bicharacters in terms of certain left and right coactions and vice versa. In Section 3.4 we introduce the most fundamental notion of quantum group homomorphism from the point of view that quantum groups encode symmetries of $\mathrm{C}^{*}$-algebras, in the form of coactions. In Section 3.5 we discuss the correspondence between bicharacters and classical group homomorphisms. Section 3.6 introduces the notion of projections on quantum groups.

\subsection{Bicharacters}

Let $\mathbb{G}=\left(A, \Delta_{A}\right)$ and $\mathbb{H}=\left(B, \Delta_{B}\right)$ be quantum groups with the reduced bicharacters $\mathrm{W}^{A} \in \mathcal{U}(\hat{A} \otimes A)$ and $\mathrm{W}^{B} \in \mathcal{U}(\hat{B} \otimes B)$, respectively.

Definition 3.1 (see also 31, Definition 3.1]). A unitary $\chi \in \mathcal{U}(\hat{A} \otimes B)$ is called a bicharacter from $\mathbb{G}$ to $\mathbb{H}$ if

$$
\begin{array}{ll}
\left(\hat{\Delta}_{A} \otimes \operatorname{id}_{B}\right) \chi=\chi_{23} \chi_{13} & \text { in } \mathcal{U}(\hat{A} \otimes \hat{A} \otimes B), \\
\left(\operatorname{id}_{\hat{A}} \otimes \Delta_{B}\right) \chi=\chi_{12} \chi_{13} & \text { in } \mathcal{U}(\hat{A} \otimes B \otimes B) .
\end{array}
$$

Remark 3.2. Definition 3.1 still makes sense if we consider $\left(\hat{A}, \hat{\Delta}_{A}\right)$ and $\left(B, \Delta_{B}\right)$ as Hopf $\mathrm{C}^{*}$-algebras. We do not expect all the nice properties of bicharacters between quantum groups in this general setting. But 
it will turn out useful in Section 3.2 while studying Hopf *-homomorphisms between universal quantum groups.

Let $\mathcal{H}_{A}$ and $\mathcal{H}_{B}$ be the generic Hilbert spaces associated to $\mathbb{G}$ and $\mathbb{H}$-Heisnberg pairs.

Lemma 3.3. A unitary $\chi \in \mathcal{U}\left(\mathcal{H}_{A} \otimes \mathcal{H}_{B}\right)$ comes from a bicharacter $\chi \in \mathcal{U}(\hat{A} \otimes B)$ (which is necesssarily unique) if and only if

$$
\begin{array}{ll}
\chi_{23} \mathbb{W}_{12}^{A}=\mathbb{W}_{12}^{A} \chi_{13} \chi_{23} & \text { in } \mathcal{U}\left(\mathcal{H}_{A} \otimes \mathcal{H}_{A} \otimes \mathcal{H}_{B}\right), \\
\mathbb{W}_{23}^{B} \chi_{12}=\chi_{12} \chi_{13} \mathbb{W}_{23}^{B} & \text { in } \mathcal{U}\left(\mathcal{H}_{A} \otimes \mathcal{H}_{B} \otimes \mathcal{H}_{B}\right) .
\end{array}
$$

Proof. The representation of $\hat{A} \otimes \hat{A} \otimes B$ on $\mathcal{H}_{A} \otimes \mathcal{H}_{A} \otimes \mathcal{H}_{B}$ is faithful. Hence a bicharacter $\chi \in \mathcal{U}(\hat{A} \otimes B)$ is determined by its image $\chi \in \mathcal{U}\left(\mathcal{H}_{A} \otimes \mathcal{H}_{B}\right)$, and (3.1) and (3.2) are equivalent to some equations of unitary operators on $\mathcal{H}_{A} \otimes \mathcal{H}_{A} \otimes \mathcal{H}_{B}$. Using 2.18, we rewrite 3.1$]$ as $\Sigma_{12}\left(\mathbb{W}_{12}^{A}\right)^{*} \chi_{23} \mathbb{W}_{12}^{A} \Sigma_{12}=\chi_{23} \chi_{13}$. This is equivalent to (3.3). A similar argument shows that (3.2) is equivalent to 3.4.

It remains to show that a unitary $\chi$ on $\mathcal{H}_{A} \otimes \mathcal{H}_{B}$ that satisfies (3.3) and (3.4) necessarily belongs to $\mathcal{U}(\hat{A} \otimes B)$. Comparing (3.4) with 2.26 we observe that $\chi$ is adapted to $\mathbb{W}^{B}$. Rewriting (3.4) as $\chi_{13}=$ $\chi_{12}^{*} \mathbb{W}_{23}^{B} \chi_{12}\left(\mathbb{W}_{23}^{B}\right)^{*}$, we see that $\chi \in \mathcal{U}\left(\mathbb{K}\left(\mathcal{H}_{A}\right) \otimes B\right)$. Equation 3.3$)$ in the form $\chi_{13}=\left(\mathbb{W}_{12}^{A}\right)^{*} \chi_{23} \mathbb{W}_{12}^{A} \chi_{23}^{*}$ shows that $\chi_{13} \in \mathcal{U}\left(\hat{A} \otimes \mathbb{K}\left(\mathcal{H}_{A}\right) \otimes B\right)$, so that $\chi \in \mathcal{U}(\hat{A} \otimes B)$ as asserted.

Example 3.4. Equations 2.8 and 2.9 show that a Hopf ${ }^{*}$-homomorphism $f: A \rightarrow B$ yields a bicharacter $V_{f}:=\left(\mathrm{id}_{\hat{A}} \otimes f\right) \mathrm{W}^{A}$. In particular, the bicharacter $\mathrm{W}^{A}$ corresponds to the identity morphism.

Remark 3.5. The criterion in Lemma 3.3 has the merit of using only the language of multiplicative unitaries and pentagon equations. But the same quantum group may be generated by different multiplicative unitaries. Since $\mathrm{W}^{A}$ only depends on $\left(A, \Delta_{A}\right)$ by 39 , bicharacters from $\mathbb{G}$ to $\mathbb{H}$ depend only on $\left(A, \Delta_{A}\right)$ and $\left(B, \Delta_{B}\right)$.

\subsubsection{Composition of bicharacters}

Now we define the composition of (concrete) bicharacters as in 31 , Lemma 2.5]. Let $\mathbb{I}=\left(C, \Delta_{C}\right)$ be another quantum group.

Definition 3.6. A unitary $\chi^{A \rightarrow C} \in \mathcal{U}(\hat{A} \otimes C)$ is called a composition of two bicharacters $\chi^{A \rightarrow B} \in \mathcal{U}(\hat{A} \otimes B)$ and $\chi^{B \rightarrow C} \in \mathcal{U}(\hat{B} \otimes C)$ if its image $\chi^{A \rightarrow C}$ in $\mathcal{U}\left(\mathcal{H}_{A} \otimes \mathcal{H}_{C}\right)$ satisfies

$$
\chi_{23}^{B \rightarrow C} \chi_{12}^{A \rightarrow B}=\chi_{12}^{A \rightarrow B} \chi_{13}^{A \rightarrow C} \chi_{23}^{B \rightarrow C} \quad \text { in } \mathcal{U}\left(\mathcal{H}_{A} \otimes \mathcal{H}_{B} \otimes \mathcal{H}_{C}\right)
$$

or, equivalently,

$$
\chi_{13}^{A \rightarrow C}=\left(\chi_{12}^{A \rightarrow B}\right)^{*} \chi_{23}^{B \rightarrow C} \chi_{12}^{A \rightarrow B}\left(\chi_{23}^{B \rightarrow C}\right)^{*} .
$$

We also briefly write $\chi^{A \rightarrow C}=\chi^{B \rightarrow C} * \chi^{A \rightarrow B}$.

Lemma 3.7. For any two bicharacters $\chi^{A \rightarrow B}$ and $\chi^{B \rightarrow C}$, there is a unique composition $\chi^{A \rightarrow C} \in \mathcal{U}(\hat{A} \otimes C)$ from $\mathbb{G}$ to $\mathbb{I}$.

Proof. We rewrite 3.5 as $\chi_{13}^{A \rightarrow C}=\left(\chi_{1 \pi}^{A \rightarrow B}\right)^{*} \chi_{\hat{\pi} 3}^{B \rightarrow C} \chi_{1 \pi}^{A \rightarrow B}\left(\chi_{\hat{\pi} 3}^{B \rightarrow C}\right)^{*}$, where $(\pi, \hat{\pi})$ is a $\mathbb{H}$-Heisenberg pair on $\mathcal{H}$. By Proposition 2.38 there is a unique $\chi^{A \rightarrow C} \in \mathcal{U}(\hat{A} \otimes C)$ satisfying (3.5).

The following computation yields $\sqrt{3.1)}$ for $\chi^{A \rightarrow C}$ :

$$
\begin{aligned}
\left(\hat{\Delta}_{A} \otimes \operatorname{id} \otimes \operatorname{id}_{C}\right) \chi_{13}^{A \rightarrow C} & =\left(\hat{\Delta}_{A} \otimes \operatorname{id} \otimes \operatorname{id}_{C}\right)\left(\left(\chi_{1 \pi}^{A \rightarrow B}\right)^{*} \chi_{\hat{\pi} 3}^{B \rightarrow C} \chi_{1 \pi}^{A \rightarrow B}\left(\chi_{\hat{\pi} 3}^{B \rightarrow C}\right)^{*}\right) \\
& =\left(\chi_{1 \pi}^{A \rightarrow B}\right)^{*}\left(\chi_{2 \pi}^{A \rightarrow B}\right)^{*} \chi_{\hat{\pi} 4}^{B \rightarrow C} \chi_{2 \pi}^{A \rightarrow B} \chi_{1 \pi}^{A \rightarrow B}\left(\chi_{\hat{\pi} 4}^{B \rightarrow C}\right)^{*} \\
& =\left(\chi_{1 \pi}^{A \rightarrow B}\right)^{*} \chi_{24}^{A \rightarrow C} \chi_{\hat{\pi} 4}^{B \rightarrow C} \chi_{1 \pi}^{A \rightarrow B}\left(\chi_{\hat{\pi} 4}^{B \rightarrow C}\right)^{*} \\
& =\chi_{24}^{A \rightarrow C}\left(\chi_{1 \pi}^{A \rightarrow B}\right)^{*} \chi_{\hat{\pi} 4}^{B \rightarrow C} \chi_{1 \pi}^{A \rightarrow B}\left(\chi_{\hat{\pi} 4}^{B \rightarrow C}\right)^{*}=\chi_{24}^{A \rightarrow C} \chi_{14}^{A \rightarrow C}
\end{aligned}
$$

The first step uses (3.5); the second step uses 3.1) for $\chi^{A \rightarrow B}$; the third step again uses (3.5); the fourth step uses that $\chi_{24}^{A \rightarrow C}$ and $\chi_{1 \pi}^{A \rightarrow B}$ commute; and the last step uses (3.5).

Similarly, one shows 3.4 . Hence $\chi^{A \rightarrow C}$ is indeed a bicharacter. 
Proposition 3.8. The composition of bicharacters is associative, and the multiplicative unitary $\mathrm{W}^{A}$ is an identity on $C$. Thus bicharacters with the above composition and locally compact quantum groups are the arrows and objects of a category, called bicharacter category.

Proof. Only associativity of the composition is non-trivial. This follows from a direct composition similar to the ones above. We omit it here because associativity follows immediately from Theorem 3.17 or from Proposition 3.28 below, which translate the composition into a different language where associativity is obvious.

\subsubsection{Properties of bicharacters}

Recall that the dual of a multiplicative unitary $\mathbb{W}^{A}$ is the multiplicative unitary $\widehat{\mathbb{W}}^{A}:=\Sigma\left(\mathbb{W}^{A}\right)^{*} \Sigma$. Correspondingly, the reduced bicharacter of the dual quantum group is $\widehat{\mathrm{W}}^{A}:=\sigma\left(\left(\mathrm{W}^{A}\right)^{*}\right)$. Here $\sigma: \hat{A} \otimes A \rightarrow$ $A \otimes \hat{A}$ is the tensor flip automorphism and $\Sigma: \mathcal{H}_{A} \otimes \mathcal{H}_{A} \rightarrow \mathcal{H}_{A} \otimes \mathcal{H}_{A}$ is the tensor flip unitary. A similar duality works for all bicharacters:

Proposition 3.9. Let $\chi \in \mathcal{U}(\hat{A} \otimes B)$ be a bicharacter from $\mathbb{G}$ to $\mathbb{H}$ and let $\chi \in \mathcal{U}\left(\mathcal{H}_{A} \otimes \mathcal{H}_{B}\right)$ be the corresponding concrete bicharacter. Then

$$
\hat{\chi}:=\sigma\left(\chi^{*}\right) \in \mathcal{U}(B \otimes \hat{A}) \quad \text { and } \quad \widehat{\chi}:=\Sigma \chi^{*} \Sigma \in \mathcal{U}\left(\mathcal{H}_{B} \otimes \mathcal{H}_{A}\right)
$$

are a bicharacter from $\widehat{\mathbb{H}}$ to $\widehat{\mathbb{G}}$ and the corresponding concrete bicharacter. Here we identify the bidual of $\mathbb{H}=\left(B, \Delta_{B}\right)$ again with $\mathbb{H}=\left(B, \Delta_{B}\right)$. This duality is a contravariant functor on the bicharacter category.

Proof. We check 3.1 for $\hat{\chi}$ using 3.2 for $\chi$ :

$$
\left(\Delta_{B} \otimes \operatorname{id}_{\hat{A}}\right) \sigma\left(\chi^{*}\right)=\sigma_{23} \sigma_{12}\left(\left(\operatorname{id}_{\hat{A}} \otimes \Delta_{B}\right) \chi^{*}\right)=\sigma_{23} \sigma_{12}\left(\chi_{13}^{*} \chi_{12}^{*}\right)=\sigma\left(\chi^{*}\right)_{23} \cdot \sigma\left(\chi^{*}\right)_{13} .
$$

A similar computation yields $(3.2)$ for $\hat{\chi}$. A quantum group and its dual are canonically represented on the same Hilbert space, and the flip $\sigma$ on operators is implemented by conjugating by $\Sigma$. Hence $\widehat{\chi}:=\Sigma \chi^{*} \Sigma$.

Functoriality follows from the following computation:

$$
\widehat{\chi_{13}^{A \rightarrow C}}=\sigma_{13}\left(\chi_{\hat{\pi} 3}^{B \rightarrow C}\left(\chi_{1 \pi}^{A \rightarrow B}\right)^{*}\left(\chi_{\hat{\pi} 3}^{B \rightarrow C}\right)^{*} \chi_{1 \pi}^{A \rightarrow B}\right)=\widehat{\chi_{1 \hat{\pi}}^{B \rightarrow C}} * \widehat{\chi_{\pi 3}^{A \rightarrow B}} \widehat{\chi_{1 \hat{\pi}}^{B \rightarrow C}}{\widehat{\chi_{\pi 3}^{A \rightarrow B}}}^{*},
$$

where $(\pi, \hat{\pi})$ is an $\mathbb{H}$-Heisenberg pair.

The following result generalises 39, Lemma 40] and is proved by the same idea.

Proposition 3.10. Let $\chi \in \mathcal{U}(\hat{A} \otimes B)$ be a bicharacter. Then

$$
\begin{aligned}
& \left(\mathrm{R}_{\hat{A}} \otimes \mathrm{R}_{B}\right)(\chi)=\chi, \\
& \left(\tau_{t}^{\hat{A}} \otimes \tau_{t}^{B}\right)(\chi)=\chi \quad \text { for all } t \in \mathbb{R} .
\end{aligned}
$$

Proof. Let $\varphi \in \hat{A}_{*}$ and $\psi \in B_{*}$ be entire analytic for $\left(\tau_{t}^{\hat{A}}\right)$ and $\left(\tau_{t}^{B}\right)$, respectively. Let $\varphi_{t}:=\varphi \circ \tau_{t}^{\hat{A}}$ and $\psi_{t}:=\psi \circ \tau_{t}^{B}$ for all $t \in \mathbb{R}$. Analytic continuation yields

$$
\varphi_{z+z^{\prime}}=\varphi_{z} \circ \tau_{z^{\prime}}^{\hat{A}}, \quad \text { and } \quad \psi_{z+z^{\prime}}=\psi_{z} \circ \tau_{z^{\prime}}^{B} \quad \text { for all } z, z^{\prime} \in \mathbb{C} .
$$

Polar decomposition of the antipodes $\kappa_{\hat{A}}$ and $\kappa_{B}(49$, Theorem 1.5]) shows that

$$
\varphi_{z} \circ \kappa_{\hat{A}}=\varphi_{z+\mathrm{i} / 2} \circ \mathrm{R}_{\hat{A}}, \quad \text { and } \quad \psi_{z} \circ \kappa_{B}=\psi_{z+\mathrm{i} / 2} \circ \mathrm{R}_{B}
$$

Let $\bar{\kappa}_{B}$ be the closure of $\kappa_{B}$ with respect to the strict topology on $\mathcal{M}(B)$. Then 49 , Theorem 1.6(4)] yields

$$
\bar{\kappa}_{B}(\omega \otimes \mathrm{id}) \chi=(\omega \otimes \mathrm{id})\left(\chi^{*}\right)
$$

for all $\omega \in \hat{A}_{*}$. Applying $\psi_{z}$ to both sides and using that $\omega$ is arbitrary, we get

$$
\left(\mathrm{id} \otimes \psi_{z+\mathrm{i} / 2} \circ \mathrm{R}_{B}\right) \chi=\left(\mathrm{id} \otimes \psi_{z}\right)\left(\chi^{*}\right) .
$$


Interchanging the roles of $B$ and $\hat{A}$ and replacing $\chi$ by $\Sigma \chi^{*} \Sigma$ and $\psi$ by $\varphi$, we get

$$
\left(\varphi_{z+\mathrm{i} / 2} \circ R_{\hat{A}} \otimes \mathrm{id}\right)\left(\chi^{*}\right)=\left(\varphi_{z} \otimes \mathrm{id}\right) \chi .
$$

Both formulas together yield

$$
\begin{array}{r}
\left(\varphi_{z+\mathrm{i} / 2} \otimes \psi_{z+\mathrm{i} / 2}\right) \circ\left(\mathrm{R}_{\hat{A}} \otimes \mathrm{R}_{B}\right)(\chi)=\left(\varphi_{z+\mathrm{i} / 2} \circ \mathrm{R}_{\hat{A}} \otimes \psi_{z+\mathrm{i} / 2} \circ \mathrm{R}_{B}\right)(\chi) \\
=\left(\varphi_{z+\mathrm{i} / 2} \circ \mathrm{R}_{\hat{A}} \otimes \psi_{z}\right)\left(\chi^{*}\right)=\psi_{z}\left(\varphi_{z+\mathrm{i} / 2} \circ \mathrm{R}_{\hat{A}} \otimes \mathrm{id}\right)\left(\chi^{*}\right)=\left(\varphi_{z} \otimes \psi_{z}\right)(\chi) .
\end{array}
$$

Inserting $\varphi \circ \kappa_{\hat{A}}$ and $\psi \circ \kappa_{B}$ into $(3.8)$ instead of $\varphi$ and $\psi$ yields

$$
\left(\varphi_{z+\mathrm{i}} \otimes \psi_{z+\mathrm{i}}\right)(\chi)=\left(\varphi_{z+\mathrm{i} / 2} \otimes \psi_{z+\mathrm{i} / 2}\right) \circ\left(\mathrm{R}_{\hat{A}} \otimes \mathrm{R}_{B}\right)(\chi)=\left(\varphi_{z} \otimes \psi_{z}\right)(\chi) .
$$

This shows that $\left(\varphi_{z} \otimes \psi_{z}\right)(\chi)$ is a periodic function of period i. Being bounded as well, Liouville's Theorem shows that it is constant, that is,

$$
\left(\varphi_{z} \otimes \psi_{z}\right)(\chi)=(\varphi \otimes \psi)(\chi)
$$

for all $z \in \mathbb{C}$. Putting $z=-\mathrm{i} / 2$ in $(3.8)$ and using $(3.9)$ yields

$$
(\varphi \otimes \psi) \circ\left(\mathrm{R}_{\hat{A}} \otimes \mathrm{R}_{B}\right)(\chi)=(\varphi \otimes \psi)(\chi) .
$$

This proves $\left(\mathrm{R}_{\hat{A}} \otimes \mathrm{R}_{B}\right)(\chi)=\chi$. Finally, 3.9$)$ also yields $\left(\tau_{t}^{\hat{A}} \otimes \tau_{t}^{B}\right)(\chi)=\chi$ for all $t \in \mathbb{R}$.

Let $Q_{A}$ and $Q_{B}$ be the positive, self-adjoint operators from the definition of manageable multiplicative unitaries of the quantum groups $\mathbb{G}$ and $\mathbb{H}$.

Corollary 3.11. $\chi \in \mathcal{U}\left(\mathcal{H}_{A} \otimes \mathcal{H}_{B}\right)$ commutes with $Q_{A} \otimes Q_{B}$.

Example 3.12. We give an interesting example of a concrete bicharacter from the definition of modular multiplicative unitaries.

Let $\mathbb{G}=\left(A, \Delta_{A}\right)$ be a quantum group generated by a modular multiplicative unitary $\mathbb{W}$ on $\mathcal{H} \otimes \mathcal{H}$. The opposite quantum group $\left(A^{\text {op }}, \Delta_{A}\right)$ is generated by a modular multiplicative unitary acting on $\overline{\mathcal{H}} \otimes \overline{\mathcal{H}}$ for the complex-conjugate Hilbert space $\overline{\mathcal{H}}$. The unitary operator $\left(\mathbb{W}^{*}\right)^{\mathrm{T} \otimes \mathrm{T}}$ on $\overline{\mathcal{H}} \otimes \overline{\mathcal{H}}$ is multiplicative and gives rise to the quantum group $\left(\bar{A}, \bar{\Delta}_{A}\right)$ with

$$
\bar{A}:=\left\{a^{\top}: a \in A\right\} \quad \text { and } \quad \bar{\Delta}_{A}\left(a^{\top}\right):=\left(\Delta_{A}(a)\right)^{\top \otimes \top} .
$$

The quantum group $\left(\bar{A}, \bar{\Delta}_{A}\right)$ is isomorphic to $\left(A^{\text {op }}, \Delta_{A}\right)$. Thus the dual $\left(\hat{\bar{A}}_{1}, \hat{\bar{\Delta}}_{A}\right)$ is isomorphic to the dual of the opposite quantum group $\left(\widehat{A^{\mathrm{op}}}, \hat{\Delta}_{A}\right)$, where

$$
\hat{\bar{A}}=\left\{\hat{a}^{\top}: \hat{a} \in \hat{A}\right\} \quad \text { and } \quad \hat{\bar{\Delta}}_{A}\left(\hat{a}^{\top}\right)=(\hat{\Delta}(\hat{a}))^{\mathrm{T} \otimes \mathrm{T}} .
$$

Recall the operator $\widetilde{\mathbb{W}}$ from the definition of a modular multiplicative unitary. Theorem 2.13(6)|(ii) and (3.6) yield $\widetilde{\mathbb{W}}^{*}=\mathrm{W}^{\top \otimes \mathrm{R}_{A}}=\mathrm{W}^{\mathrm{R}_{\hat{A}}}{ }^{\mathrm{T} \otimes \mathrm{id}} \in \mathcal{U}(\overline{\mathcal{H}} \otimes \mathcal{H})$. Hence 3.11 yields $\widetilde{\mathrm{W}} \in \mathcal{U}(\hat{\bar{A}} \otimes A)$. We compute

$$
\begin{aligned}
\left(\hat{\bar{\Delta}}_{A} \otimes \mathrm{id}_{A}\right) \widetilde{\mathrm{W}}^{*} & =\left(\left(\hat{\Delta}_{A} \otimes \mathrm{id}_{A}\right) \mathrm{W}\right)^{\mathrm{T} \otimes \mathrm{T} \otimes \mathrm{R}_{A}}=\left(\mathrm{W}_{23} \mathrm{~W}_{13}\right)^{\mathrm{T} \otimes \mathrm{T} \otimes \mathrm{R}_{A}}=\widetilde{\mathrm{W}}_{13}^{*} \widetilde{\mathrm{W}}_{23}^{*} . \\
\left(\mathrm{id}_{\hat{A}} \otimes \sigma \circ \Delta_{A}\right) \widetilde{\mathrm{W}}^{*} & =\left(\left(\mathrm{id}_{\hat{A}} \otimes \sigma \circ \Delta_{A}\right) \mathrm{W}\right)^{\mathrm{T} \otimes \mathrm{R}_{A} \otimes \mathrm{R}_{A}}=\left(\mathrm{W}_{12} \mathrm{~W}_{13}\right)^{\mathrm{T} \otimes \mathrm{R}_{A} \otimes \mathrm{R}_{A}}=\widetilde{\mathrm{W}}_{13}^{*} \widetilde{\mathrm{W}}_{12}^{*} .
\end{aligned}
$$

Thus $\widetilde{\mathrm{W}}$ is a bicharacter from $\left(\bar{A}, \bar{\Delta}_{A}\right)$ to $\left(A, \Delta_{A}^{\mathrm{op}}\right)$. 


\subsubsection{Manageability of bicharacters}

Lemma 3.3 and Proposition 3.9 show that $\chi \in \mathcal{U}\left(\mathcal{H}_{A} \otimes \mathcal{H}_{B}\right)$ and $\widehat{\chi} \in \mathcal{U}\left(\mathcal{H}_{B} \otimes \mathcal{H}_{A}\right)$ are adapted to the multiplicative unitaries $\mathbb{W}^{B}$ and $\widehat{\mathbb{W}}^{B}$. Assume that $\mathbb{W}^{A}$ and $\mathbb{W}^{B}$ are manageable multiplicative unitaries (if they are modular consider the corresponding manageable multiplicative unitaries as described in 38$]$ ).

By (2.27), adaptation to $\mathbb{W}^{B}$ yields manageability for $\chi \in \mathcal{U}\left(\mathcal{H}_{A} \otimes \mathcal{H}_{B}\right)$ : there is a unitary $\widetilde{\chi} \in$ $\mathcal{U}\left(\overline{\mathcal{H}}_{A} \otimes \mathcal{H}_{B}\right)$ such that:

$$
(x \otimes u|\chi| z \otimes y)=\left(\bar{z} \otimes Q_{B} u|\tilde{\chi}| \bar{x} \otimes Q_{B}^{-1} y\right),
$$

for all $x, z \in \mathcal{H}_{A}, u \in \mathcal{D} \operatorname{Dom}\left(Q_{B}\right)$ and $y \in \mathcal{D o m}\left(Q_{B}^{-1}\right)$. Moreover, $\widetilde{\chi}^{*}:=\chi^{\top \otimes R_{B}} \in \mathcal{U}\left(\overline{\mathcal{H}}_{A} \otimes \mathcal{H}_{B}\right)$.

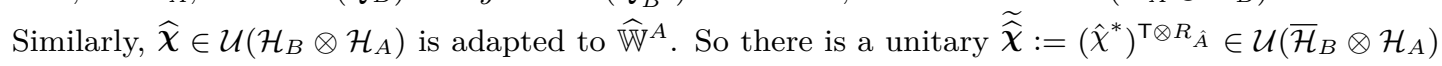
satisfying

$$
(x \otimes u|\widehat{\chi}| z \otimes y)=\left(\bar{z} \otimes Q_{A} u|\widetilde{\widehat{\chi}}| \bar{x} \otimes Q_{A}^{-1} y\right),
$$

for all $x, z \in \mathcal{H}_{B}, u \in \mathcal{D} \operatorname{om}\left(Q_{A}\right)$ and $y \in \mathcal{D} \operatorname{om}\left(Q_{A}^{-1}\right)$. Equation 3.6 shows that $\left(\Sigma\left(\widetilde{\chi}^{*}\right) \Sigma\right)^{\mathrm{T} \otimes \mathrm{T}}=\widetilde{\widehat{\chi}}$.

Lemma 3.13. $\widetilde{\chi}$ and $Q_{A}^{\top} \otimes Q_{B}^{-1}$ commute.

Proof. Since $\chi$ commutes with $Q_{A} \otimes Q_{B}$, in 3.12 we can replace $x, u, z$ and $y$ by $Q_{A}^{\mathrm{it}}(x), Q_{B}^{\mathrm{it}}(u), Q_{A}^{\mathrm{it}}(z)$ and $Q_{B}^{\mathrm{it}}(y)$, respectively, for all $t \in \mathbb{R}$. Thus we obtain

$$
\left(\bar{z} \otimes Q_{B} u|\tilde{\chi}| \bar{x} \otimes Q_{B}^{-1} y\right)=\left(\left[Q_{A}^{\top}\right]^{-\mathrm{i} t} \bar{z} \otimes Q_{B}^{\mathrm{it}} Q_{B} u|\tilde{\chi}|\left[Q_{A}^{\top}\right]^{-\mathrm{i} t} \bar{x} \otimes Q_{B}^{\mathrm{i} t} Q_{B}^{-1} u\right)
$$

and therefore $\widetilde{\chi}=\left(\left[Q_{A}^{\top}\right]^{\mathrm{i} t} \otimes Q_{B}^{-\mathrm{i} t}\right) \widetilde{\chi}\left(\left[Q_{A}^{\top}\right]^{\mathrm{i} t} \otimes Q_{B}^{-\mathrm{i} t}\right)$ for all $t \in \mathbb{R}$.

\subsection{Passage to universal quantum groups}

In this section we show that our quantum group homomorphisms are equivalent to Hopf *-homomorphisms between the associated universal quantum groups, which were previously suggested as a suitable notion of quantum group homomorphism (see 22. Section 12]). Proposition 2.42 shows that every reduced bicharacter admits a unique bi-lift to a universal bicharacter. Thus modular (or manageable) multiplicative unitaries are basic in the notation of [31, Definition 2.3].

Proposition 3.14. Let $\left(B, \Delta_{B}\right)$ be a Hopf $\mathrm{C}^{*}$-algebra. Bicharacters in $\mathcal{U}(\hat{A} \otimes B)$ correspond bijectively to Hopf ${ }^{*}$-homomorphisms from $\mathbb{G}^{\mathrm{u}}=\left(A^{\mathrm{u}}, \Delta_{A^{\mathrm{u}}}\right)$ to $\left(B, \Delta_{B}\right)$.

Proof. A Hopf *-homomorphism $\varphi: A^{\mathrm{u}} \rightarrow B$ is also a morphism from $A^{\mathrm{u}}$ to $B$ and thus corresponds to a corepresentation $\chi \in \mathcal{U}(\hat{A} \otimes B)$ of $\widehat{\mathbb{G}}$ on $B$, which is determined by the condition 2.40$):\left(\operatorname{id}_{\hat{A}} \otimes \varphi\right)(\mathcal{V})=$ $\chi$. The Hopf ${ }^{*}$-homomorphisms $\Delta_{B} \circ \varphi: A^{\mathrm{u}} \rightarrow B \otimes B$ and $(\varphi \otimes \varphi) \circ \Delta_{A^{\mathrm{u}}}: A^{\mathrm{u}} \rightarrow B \otimes B$ correspond to the corepresentations $\left(\operatorname{id}_{\hat{A}} \otimes \Delta_{B}\right) \chi$ and $\chi_{12} \chi_{13}$, that is, $\operatorname{id}_{\hat{A}} \otimes\left(\Delta_{B} \circ \varphi\right)(\mathcal{V})=\left(\operatorname{id}_{\hat{A}} \otimes \Delta_{B}\right) \chi$ and $\left(\operatorname{id}_{\hat{A}} \otimes(\varphi \otimes \varphi) \circ \Delta_{A^{\mathrm{u}}}\right)(\mathcal{V})=\chi_{12} \chi_{13}$ because $\mathcal{V}$ is a bicharacter. Thus a morphism $\varphi: A^{\mathrm{u}} \rightarrow B$ is a Hopf ${ }^{*}$-homomorphism if and only if the corepresentation $\chi$ also satisfies $\left(\operatorname{id}_{\hat{A}} \otimes \Delta_{B}\right) \chi=\chi_{12} \chi_{13}$. That is, $\chi$ is a bicharacter.

Corollary 3.15. Any Hopf ${ }^{*}$-homomorphism $\varphi: A^{\mathrm{u}} \rightarrow B$ induces a dual Hopf ${ }^{*}$-homomorphism $\hat{\varphi}: \hat{B}^{\mathrm{u}} \rightarrow \hat{A}$ if $\left(B, \Delta_{B}\right)$ is also a quantum group.

Proof. By Proposition 3.14 a Hopf *-homomorphism $\varphi: A^{\mathrm{u}} \rightarrow B$ corresponds to a bicharacter $\chi$ in $\mathcal{U}(\hat{A} \otimes B)$. By Proposition $3.9 \sigma\left(V^{*}\right)$ is a bicharacter from $\hat{B}$ to $\hat{A}$, which yields a Hopf ${ }^{*}$-homomorphism $\varphi: \hat{B}^{\mathrm{u}} \rightarrow \hat{A}$ by Proposition 3.14

Now we are going to show that Hopf ${ }^{*}$-homomorphisms $\mathbb{G}^{\mathrm{u}} \rightarrow \mathbb{H}$ lift uniquely to Hopf *-homomorphisms from $\mathbb{G}^{\mathrm{u}}$ to $\mathbb{H}^{\mathrm{u}}$. Together with Proposition 3.14 this yields a bijection between homomorphisms of quantum groups in our sense and Hopf *-homomorphisms between the associated universal quantum groups. The main ingredient is the universal bicharacter $\mathcal{W} \in \mathcal{U}\left(\hat{A}^{\mathrm{u}} \otimes A^{\mathrm{u}}\right)$ from Proposition 2.42 
Proposition 3.16. A bicharacter in $\mathcal{U}(\hat{A} \otimes B)$ lifts uniquely to a bicharacter in $\mathcal{U}\left(\hat{A}^{\mathrm{u}} \otimes B^{\mathrm{u}}\right)$ and hence to bicharacters in $\mathcal{U}\left(\hat{A} \otimes B^{\mathrm{u}}\right)$ and $\mathcal{U}\left(\hat{A}^{\mathrm{u}} \otimes B\right)$.

Proof. These liftings are unique by Lemma 2.41 It remains to prove existence. Let $\chi \in \mathcal{U}(\hat{A} \otimes B)$ be a bicharacter. By Proposition 3.14 it corresponds to a Hopf *-homomorphism $\varphi: A^{\mathrm{u}} \rightarrow B$. Let $\mathcal{W}^{A} \in \mathcal{U}\left(\hat{A}^{\mathrm{u}} \otimes A^{\mathrm{u}}\right)$ be the universal bicharacter of $\mathbb{G}$. Then $\chi^{\prime}:=\left(\mathrm{id}_{\hat{A}^{\mathrm{u}}} \otimes \varphi\right) \mathcal{W}^{A} \in \mathcal{U}\left(\hat{A}^{\mathrm{u}} \otimes B\right)$ is a bicharacter that lifts $\chi$. Now $\sigma\left(\chi^{\prime}\right)^{*} \in \mathcal{U}\left(B \otimes \hat{A}^{\mathrm{u}}\right.$ ) is again a bicharacter (see Proposition 3.9). Repeating the above step we lift it to a bicharacter $\chi^{\prime \prime}$ in $\mathcal{U}\left(B^{\mathrm{u}} \otimes \hat{A}^{\mathrm{u}}\right)$. Then $\sigma\left(\chi^{\prime \prime}\right)^{*}$ is the desired lifting of $\chi$.

Recall that bicharacters form a category and that duality is a functor on this category. Hopf *-homomorphisms $A^{\mathrm{u}} \rightarrow B^{\mathrm{u}}$ also form the arrows of a category.

Theorem 3.17. There is an isomorphism between the categories of locally compact quantum groups with bicharacters from $\mathbb{G}$ to $\mathbb{H}$ and with Hopf ${ }^{*}$-homomorphisms $A^{\mathrm{u}} \rightarrow B^{\mathrm{u}}$ as morphisms $\mathbb{G} \rightarrow \mathbb{H}$, respectively. The bicharacter associated to a Hopf ${ }^{*}$-homomorphism $\varphi: A^{\mathrm{u}} \rightarrow B^{\mathrm{u}}$ is $\left(\Lambda_{\hat{A}} \otimes \Lambda_{B} \varphi\right)\left(\mathcal{W}^{A}\right) \in \mathcal{U}(\hat{A} \otimes B)$.

Furthermore, the duality on the level of bicharacters corresponds to the duality $\varphi \mapsto \hat{\varphi}$ on Hopf ${ }^{*}$-homomorphisms, where $\hat{\varphi}: \hat{B}^{\mathrm{u}} \rightarrow \hat{A}^{\mathrm{u}}$ is the unique Hopf ${ }^{*}$-homomorphism with $\left(\hat{\varphi} \otimes \operatorname{id}_{B^{u}}\right)\left(\mathcal{W}^{B}\right)=$ $\left(\operatorname{id}_{\hat{A}^{\mathrm{u}}} \otimes \varphi\right)\left(\mathcal{W}^{A}\right)$.

Proof. Propositions 3.14 and 3.16 yield bijections from Hopf ${ }^{*}$-homomorphisms $A^{\mathrm{u}} \rightarrow B^{\mathrm{u}}$ to bicharacters from $\mathbb{G}$ to $\mathbb{H}^{\mathrm{u}}$ and on to bicharacters from $\mathbb{G}$ to $\mathbb{H}$. We must check that this bijection preserves the compositions and the duality. We first turn to the duality because we need this to establish the compatibility with compositions.

Let $\varphi: A^{\mathrm{u}} \rightarrow B^{\mathrm{u}}$ be a Hopf ${ }^{*}$-homomorphism. Let $\chi:=\left(\Lambda_{\hat{A}} \otimes \Lambda_{B} \varphi\right)\left(\mathcal{W}^{A}\right) \in \mathcal{U}(\hat{A} \otimes B)$ be the associated bicharacter. The duality on the level of bicharacters yields the bicharacter $\sigma\left(\chi^{*}\right) \in \mathcal{U}(B \otimes \hat{A})$ from $\widehat{\mathbb{H}}$ to $\widehat{\mathbb{G}}$. This corresponds to a unique Hopf ${ }^{*}$-homomorphism $\hat{\varphi}: \hat{B}^{\mathrm{u}} \rightarrow \hat{A}^{\mathrm{u}}$ with $\left(\Lambda_{B} \otimes \Lambda_{\hat{A}} \hat{\varphi}\right)\left(\mathcal{W}^{\hat{B}}\right)=\sigma(\chi)^{*}$. Now we use $\mathcal{W}^{\hat{B}}=\sigma\left(\mathcal{W}^{B}\right)^{*}$ to rewrite this as

$$
\left(\Lambda_{\hat{A}} \otimes \Lambda_{B} \varphi\right)\left(\mathcal{W}^{A}\right)=\left(\Lambda_{\hat{A}} \hat{\varphi} \otimes \Lambda_{B}\right)\left(\mathcal{W}^{B}\right) .
$$

Both $\left(\operatorname{id}_{\hat{A}^{u}} \otimes \varphi\right)\left(\mathcal{W}^{A}\right)$ and $\left(\hat{\varphi} \otimes \operatorname{id}_{B^{u}}\right)\left(\mathcal{W}^{B}\right)$ are bicharacters. Applying Lemma 2.41 to both tensor factors, we get first $\left(\operatorname{id}_{\hat{A}^{\mathrm{u}}} \otimes \Lambda_{B} \varphi\right)\left(\mathcal{W}^{A}\right)=\left(\hat{\varphi} \otimes \Lambda_{B}\right)\left(\mathcal{W}^{B}\right)$ and then $\left(\operatorname{id}_{\hat{A}^{u}} \otimes \varphi\right)\left(\mathcal{W}^{A}\right)=\left(\hat{\varphi} \otimes \operatorname{id}_{B^{u}}\right)\left(\mathcal{W}^{B}\right)$. This yields the asserted description of duality.

Now let $\varphi: A^{\mathrm{u}} \rightarrow B^{\mathrm{u}}$ and $\psi: B^{\mathrm{u}} \rightarrow C^{\mathrm{u}}$ be Hopf ${ }^{*}$-homomorphisms and let $\chi^{A \rightarrow B} \in \mathcal{U}(\hat{A} \otimes B)$ and $\chi^{B \rightarrow C} \in \mathcal{U}(\hat{B} \otimes C)$ be the corresponding bicharacters,

$$
\begin{aligned}
& \chi^{B \rightarrow C}=\left(\Lambda_{\hat{B}} \otimes \Lambda_{C} \psi\right) \mathcal{W}^{B}=\left(\operatorname{id}_{\hat{B}} \otimes \Lambda_{C} \psi\right) \mathcal{V}^{B}, \\
& \chi^{A \rightarrow B}=\left(\Lambda_{\hat{A}} \hat{\varphi} \otimes \Lambda_{B}\right) \mathcal{W}^{B}=\left(\Lambda_{\hat{A}} \hat{\varphi} \otimes \operatorname{id}_{B}\right) \tilde{\mathcal{V}}^{B},
\end{aligned}
$$

where we use the dual quantum group homomorphism $\hat{\varphi}: \hat{B}^{\mathrm{u}} \rightarrow \hat{A}^{\mathrm{u}}$. Let $(\pi, \hat{\pi})$ be an $\mathbb{H}$-Heisenberg pair acting on $\mathcal{H}$. Then

$$
\begin{aligned}
\left(\chi^{B \rightarrow C} * \chi^{A \rightarrow B}\right)_{13} & =\left(\chi_{1 \pi}^{A \rightarrow B}\right)^{*} \chi_{\tilde{\pi} 3}^{B \rightarrow C} \chi_{1 \pi}^{A \rightarrow B}\left(\chi_{\tilde{\pi} 3}^{B \rightarrow C}\right)^{*} \\
& =\left(\Lambda_{\hat{A}} \hat{\varphi} \otimes \operatorname{id} \otimes \Lambda_{C} \psi\right)\left(\left(\tilde{\mathcal{V}}_{1 \pi}^{B}\right)^{*} \mathcal{V}_{\hat{\pi} 3}^{B} \tilde{\mathcal{V}}_{1 \pi}^{B}\left(\mathcal{V}_{\hat{\pi} 3}^{B}\right)^{*}\right)=\left(\Lambda_{\hat{A}} \hat{\varphi} \otimes \operatorname{id} \otimes \Lambda_{C} \psi\right)\left(\mathcal{W}_{13}^{B}\right)
\end{aligned}
$$

by Proposition 2.42 Thus

$$
\begin{aligned}
\chi^{B \rightarrow C} * \chi^{A \rightarrow B}=\left(\Lambda_{\hat{A}} \hat{\varphi} \otimes \Lambda_{C} \psi\right)\left(\mathcal{W}^{B}\right)= & \left(\Lambda_{\hat{A}} \otimes \Lambda_{C} \psi\right) \circ\left(\hat{\varphi} \otimes \operatorname{id}_{B^{u}}\right)\left(\mathcal{W}^{B}\right) \\
& =\left(\Lambda_{\hat{A}} \otimes \Lambda_{C} \psi\right) \circ\left(\operatorname{id}_{\hat{A}^{u}} \otimes \varphi\right)\left(\mathcal{W}^{A}\right)=\left(\Lambda_{\hat{A}} \otimes \Lambda_{C}(\psi \circ \varphi)\right)\left(\mathcal{W}^{A}\right) .
\end{aligned}
$$

Hence $\chi^{B \rightarrow C} * \chi^{A \rightarrow B}$ is the bicharacter associated to $\psi \circ \varphi$. Thus our bijection is compatible with compositions.

Remark 3.18. Proposition 2.42 or more generally Proposition 3.16 shows that modular multiplicative unitaries are basic in the notation of 31, Definition 2.3]. Thus Theorem 3.17 is comparable to 31, Theorem 4.9]. 


\subsection{Right and left coactions}

Let $\mathbb{G}=\left(A, \Delta_{A}\right)$ and $\mathbb{H}=\left(B, \Delta_{B}\right)$ be quantum groups with their reduced bicharacters $\mathrm{W}^{A} \in \mathcal{U}(\hat{A} \otimes A)$ and $\mathrm{W}^{B} \in \mathcal{U}(\hat{B} \otimes B)$.

Definition 3.19. A right quantum group homomorphism $\mathbb{G} \rightarrow \mathbb{H}$ is a morphism $\Delta_{R}: A \rightarrow A \otimes B$ for which the following diagrams commute:

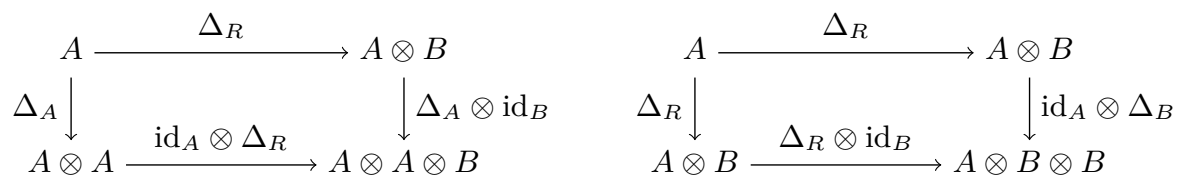

The second diagram in 3.14 means that $\Delta_{R}$ is an $\mathbb{H}$-comodule structure on $A$.

Example 3.20. A Hopf ${ }^{*}$-homomorphism $\varphi: A \rightarrow \mathcal{M}(B)$ yields a right quantum group homomorphism by $\Delta_{R}:=\left(\operatorname{id}_{A} \otimes \varphi\right) \Delta_{A}$.

Theorem 3.21. For any right quantum group homomorphism $\Delta_{R}: A \rightarrow A \otimes B$, there is a unique unitary $\chi \in \mathcal{U}(\hat{A} \otimes B)$ with

$$
\left(\operatorname{id}_{\hat{A}} \otimes \Delta_{R}\right)\left(\mathrm{W}^{A}\right)=\mathrm{W}_{12}^{A} \chi_{13}
$$

This unitary is a bicharacter.

Conversely, let $\chi$ be a bicharacter from $\mathbb{G}$ to $\mathbb{H}$, and let $\chi \in \mathcal{U}\left(\mathcal{H}_{A} \otimes \mathcal{H}_{B}\right)$ be the corresponding concrete bicharacter. Then

$$
\Delta_{R}(a):=\chi(a \otimes 1) \chi^{*} \quad \text { for all } a \in A
$$

defines a right quantum group homomorphism from $\mathbb{G}$ to $\mathbb{H}$.

These two maps between bicharacters and right quantum group homomorphisms are inverse to each other.

Proof. First we check that $\bar{\chi}:=\left(\mathrm{W}^{A}\right)_{12}^{*} \cdot\left(\operatorname{id}_{\hat{A}} \otimes \Delta_{R}\right)\left(\mathrm{W}^{A}\right)$ belongs to $\mathcal{U}(\hat{A} \otimes 1 \otimes B)$, that is, $\bar{\chi}=\chi_{13}$ for some $\chi \in \mathcal{U}(\hat{A} \otimes B)$. This is the unique $\chi$ that verifies 3.15 . We compute

$$
\begin{aligned}
\left(\operatorname{id}_{\hat{A}} \otimes \Delta_{A} \otimes \mathrm{id}_{B}\right) \bar{\chi} & =\left(\left(\mathrm{W}^{A}\right)_{13}^{*}\left(\mathrm{~W}^{A}\right)_{12}^{*}\right) \cdot\left(\left(\mathrm{id}_{\hat{A}} \otimes\left(\Delta_{A} \otimes \mathrm{id}_{B}\right) \Delta_{R}\right) \mathrm{W}^{A}\right) \\
& =\left(\left(\mathrm{W}^{A}\right)_{13}^{*}\left(\mathrm{~W}^{A}\right)_{12}^{*}\right) \cdot\left(\left(\mathrm{id}_{\hat{A}} \otimes\left(\mathrm{id}_{A} \otimes \Delta_{R}\right) \Delta_{A}\right) \mathrm{W}^{A}\right) \\
& =\left(\left(\mathrm{W}^{A}\right)_{13}^{*}\left(\mathrm{~W}^{A}\right)_{12}^{*}\right) \cdot\left(\left(\mathrm{id}_{\hat{A}} \otimes \mathrm{id}_{A} \otimes \Delta_{R}\right) \mathrm{W}_{12}^{A} \mathrm{~W}_{13}^{A}\right) \\
& =\left(\mathrm{W}^{A}\right)_{13}^{*} \cdot\left(\left(\mathrm{id}_{\hat{A}} \otimes \Delta_{R}\right) \mathrm{W}^{A}\right)_{134}=\bar{\chi}_{13},
\end{aligned}
$$

the first equality is the definition of $\bar{\chi}$ and (2.8), the second one uses the first diagram in (3.14), the third one uses 2.8 and the last two are trivial. Now Lemma 2.28 yields $\bar{\chi}=\chi_{13}$ for some $\chi \in \mathcal{U}(A \otimes B)$.

Next we verify that $\chi$ is a bicharacter. We check 3.1 :

$$
\begin{aligned}
\left(\left(\hat{\Delta}_{A} \otimes \mathrm{id}_{B}\right) \chi\right)_{124} & =\left(\hat{\Delta}_{A} \otimes \mathrm{id}_{A} \otimes \mathrm{id}_{B}\right)\left(\left(\mathrm{W}^{A}\right)_{12}^{*} \cdot\left(\mathrm{id}_{\hat{A}} \otimes \Delta_{R}\right)\left(\mathrm{W}^{A}\right)\right) \\
& =\left(\left(\hat{\Delta}_{A} \otimes \mathrm{id}_{A}\right)\left(\mathrm{W}^{A}\right)^{*}\right)_{123} \cdot\left(\left(\mathrm{id}_{\hat{A}} \otimes \mathrm{id}_{\hat{A}} \otimes \Delta_{R}\right)\left(\hat{\Delta}_{A} \otimes \mathrm{id}_{A}\right) \mathrm{W}^{A}\right) \\
& =\left(\mathrm{W}_{23}^{A} \mathrm{~W}_{13}^{A}\right)^{*}\left(\mathrm{id}_{\hat{C}} \otimes \mathrm{id}_{\hat{C}} \otimes \Delta_{R}\right)\left(\mathrm{W}_{23}^{A} \mathrm{~W}_{13}^{A}\right) \\
& =\left(\mathrm{W}^{A}\right)_{13}^{*}\left(\mathrm{~W}^{A}\right)_{23}^{*} \mathrm{~W}_{23}^{A} \chi_{24} \mathrm{~W}_{13}^{A} \chi_{14}=\chi_{24} \chi_{14} ;
\end{aligned}
$$

the first two equalities use 3.15 and that $\hat{\Delta}_{A}$ is a ${ }^{*}$-homomorphism; the third equality uses 2.9 ; the fourth one uses (3.15) again; and the final step uses that $\mathrm{W}_{13}$ and $\chi_{24}$ commute. The following computation yields 3.2 :

$$
\begin{aligned}
\left(\left(\operatorname{id}_{\hat{A}} \otimes \Delta_{B}\right) \chi\right)_{134} & =\left(\mathrm{W}^{A}\right)_{12}^{*}\left(\operatorname{id}_{\hat{A}} \otimes \mathrm{id}_{A} \otimes \Delta_{B}\right)\left(\mathrm{id}_{\hat{A}} \otimes \Delta_{R}\right) \mathrm{W}^{A} \\
& =\left(\mathrm{W}^{A}\right)_{12}^{*}\left(\mathrm{id}_{\hat{A}} \otimes \Delta_{R} \otimes \mathrm{id}_{B}\right)\left(\mathrm{id}_{\hat{A}} \otimes \Delta_{R}\right) \mathrm{W}^{A} \\
& =\left(\mathrm{W}^{A}\right)_{12}^{*}\left(\mathrm{id}_{\hat{A}} \otimes \Delta_{R} \otimes \mathrm{id}_{B}\right)\left(\mathrm{W}_{12}^{A} \chi_{14}\right)=\chi_{13} \chi_{14}
\end{aligned}
$$


the first equality follows from (3.15); the second one from the second diagram of (3.14); the third and fourth equalities from 3.15 .

Thus we have constructed a bicharacter $\chi$ from a right quantum group homomorphism. Conversely, let $\chi \in \mathcal{U}(\hat{A} \otimes B)$ be a bicharacter. We claim that $(3.16)$ defines a morphism from $A$ to $A \otimes B$. Recall that slices of $\mathbb{W}^{A} \in \mathcal{U}\left(\mathcal{H}_{A} \otimes \mathcal{H}_{A}\right)$ by linear functionals $\omega \in \mathbb{B}\left(\mathcal{H}_{A}\right)_{*}$ generate a dense subspace of $A$. On $a:=\left(\omega \otimes \operatorname{id}_{\mathcal{H}_{A}}\right)\left(\mathbb{W}^{A}\right)$, we compute

$$
\Delta_{R}(a)=\left(\omega \otimes \operatorname{id}_{\mathcal{H}_{A}} \otimes \operatorname{id}_{\mathcal{H}_{B}}\right)\left(\chi_{23} \mathbb{W}_{12} \chi_{23}^{*}\right)=\left(\omega \otimes \operatorname{id}_{\mathcal{H}_{A}} \otimes \operatorname{id}_{\mathcal{H}_{B}}\right)\left(\mathbb{W}_{12} \chi_{13}\right),
$$

and this belongs to $\mathcal{M}(A \otimes B)$ (as the representation of $A \otimes B$ on $\mathcal{H}_{A} \otimes \mathcal{H}_{B}$ is faithful). Thus $\Delta_{R}(A) \subseteq$ $\mathcal{M}(A \otimes B)$. It is clear from the definition that $\Delta_{R}$ is non-degenerate.

We may also rewrite the above computation as

$$
\left(\omega \otimes \operatorname{id}_{A \otimes B}\right) \circ\left(\operatorname{id}_{\hat{A}} \otimes \Delta_{R}\right)\left(\mathrm{W}^{A}\right)=\left(\omega \otimes \mathrm{id}_{A \otimes B}\right)\left(\mathrm{W}_{12}^{A} \chi_{13}\right)
$$

for all $\omega \in \mathbb{B}(\mathcal{H})_{*}$. Since $\omega$ is arbitrary, 3.15 holds for $\Delta_{R}$ and our original bicharacter $\chi$.

Now we use (3.15) to check that $\Delta_{R}$ is a right quantum group homomorphism. The first diagram in 3.14 amounts to

$$
\left(\mathrm{id}_{\hat{A}} \otimes \Delta_{A} \otimes \mathrm{id}_{B}\right)\left(\mathrm{id}_{\hat{A}} \otimes \Delta_{R}\right)\left(\mathrm{W}^{A}\right)=\left(\mathrm{id}_{\hat{A}} \otimes \mathrm{id}_{A} \otimes \Delta_{R}\right)\left(\mathrm{id}_{\hat{A}} \otimes \Delta_{A}\right)\left(\mathrm{W}^{A}\right)
$$

because slices on the first leg of $\mathrm{W}^{A}$ generate $A$. This follows from $(3.15)$ and $(2.8)$ : both sides are equal to $\mathrm{W}_{12}^{A} \mathrm{~W}_{13}^{A} \chi_{14}$. Similarly, the second diagram in 3.14 amounts to

$$
\left(\mathrm{id}_{\hat{A}} \otimes \mathrm{id}_{A} \otimes \Delta_{B}\right)\left(\operatorname{id}_{\hat{A}} \otimes \Delta_{R}\right)\left(\mathrm{W}^{A}\right)=\left(\operatorname{id}_{\hat{A}} \otimes \Delta_{R} \otimes \mathrm{id}_{B}\right)\left(\mathrm{id}_{\hat{A}} \otimes \Delta_{R}\right)\left(\mathrm{W}^{A}\right),
$$

which follows from $(3.15)$ and 3.2 because both sides are equal to $\mathrm{W}_{12}^{A} \chi_{13} \chi_{14}$.

Thus a bicharacter $\chi$ yields a right quantum group homomorphism $\Delta_{R}$. Since these are related by (3.15), we get back the original bicharacter from this right quantum group homomorphism. It only remains to check that, if we start with a right quantum group homomorphism $\Delta_{R}$, define a bicharacter by 3.15 and then a right quantum group homomorphism by (3.16), we get back the original $\Delta_{R}$. We may rewrite (3.3) as

$$
\chi_{23} \mathbb{W}_{12}^{A} \chi_{23}^{*}=\mathbb{W}_{12}^{A} \chi_{13}=\left(\mathrm{id}_{\hat{A}} \otimes \Delta_{R}\right)\left(\mathbb{W}^{A}\right),
$$

using (3.15). This implies that the original $\Delta_{R}$ satisfies (3.16) because the slices of $\mathrm{W}^{A}$ by linear functionals on $\hat{A}$ span a dense subspace of $A$.

Theorem 3.21 shows that 3.16 is independent of the choice of $\mathbb{G}$ and $\mathbb{H}$-Heisenberg pairs. Given any Heisenberg pair $(\pi, \hat{\pi})$ we identify $A$ with $\pi(A)$ and rewrite 3.16 as:

$$
\left(\pi \otimes \operatorname{id}_{B}\right) \Delta_{R}(a)=\chi_{\hat{\pi} 2}\left(\pi(a) \otimes 1_{B}\right) \chi_{\hat{\pi} 2}^{*} \quad \text { for all } a \in A .
$$

Definition 3.22. A left quantum group homomorphism from $\mathbb{G}$ to $\mathbb{H}$ is a morphism $\Delta_{L}: A \rightarrow B \otimes A$ such that the following two diagrams commute:

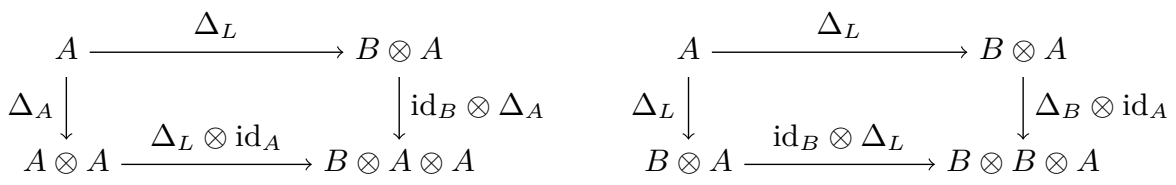

Theorem 3.23. For any left quantum group homomorphism $\Delta_{L}: A \rightarrow B \otimes A$, there is a unique unitary $\chi \in \mathcal{U}(\hat{A} \otimes B)$ with

$$
\left(\mathrm{id}_{\hat{A}} \otimes \Delta_{L}\right)\left(\mathrm{W}^{A}\right)=\chi_{12} \mathrm{~W}_{13}^{A} .
$$

This unitary is a bicharacter.

Conversely, let $\chi$ be a bicharacter from $\mathbb{G}$ to $\mathbb{H}$, let $\chi \in \mathcal{U}\left(\mathcal{H}_{A} \otimes \mathcal{H}_{B}\right)$ be the corresponding concrete bicharacter, and define $\hat{\chi}$ as in Proposition 3.9. Let $R_{A}$ and $R_{B}$ be the unitary antipodes of $\mathbb{G}$ and $\mathbb{H}$. Then $\hat{\chi}^{*}\left(1 \otimes R_{A}(a)\right) \hat{\chi} \in \mathcal{M}(B \otimes A)$ for all $a \in A$ and

$$
\Delta_{L}(a):=\left(R_{B} \otimes R_{A}\right)\left(\hat{\chi}^{*}\left(1 \otimes R_{A}(a)\right) \hat{\chi}\right) \quad \text { for all } a \in A
$$


is a left quantum group homomorphism from $\mathbb{G}$ to $\mathbb{H}$.

These two maps between bicharacters and left quantum group homomorphisms are bijective and inverse to each other.

Proof. As in the proof of Theorem 3.21 there is a unique $\chi$ satisfying $(3.19)$ and $\Delta_{L}$ is a well-defined left quantum group morphism $A \rightarrow B \otimes A$. The only point in the proof of Theorem 3.21 that must be modified is to show that $\Delta_{L}$ given by 3.20 satisfies 3.19 . We compute:

$$
\begin{aligned}
\left(\mathrm{id}_{\hat{A}} \otimes \Delta_{L}\right) \mathrm{W}^{A} & =\left(\operatorname{id}_{\hat{A}} \otimes \Delta_{L}\right)\left(\left(R_{\hat{A}} \otimes R_{A}\right) \circ \mathrm{W}^{A}\right) \\
& =\left(R_{\hat{A}} \otimes R_{B} \otimes R_{A}\right)\left(\hat{\chi}_{23}^{*} \mathbb{W}_{13}^{A} \hat{\chi}_{23}\right) \\
& =\left(R_{\hat{A}} \otimes R_{B} \otimes R_{A}\right) \circ \sigma_{23}\left(\chi_{23} \mathbb{W}_{12}^{A} \chi_{23}^{*}\right) \\
& =\left(R_{\hat{A}} \otimes R_{B} \otimes R_{A}\right)\left(\mathrm{W}_{13}^{A} \chi_{12}\right)=\chi_{12} \mathrm{~W}_{13}
\end{aligned}
$$

the first step uses Proposition 3.10 for $\mathrm{W}^{A}$, the second one uses $(3.20)$, the third one is trivial, the fourth one uses 3.3 , and the last one follows from Proposition 3.10 and the antimultiplicativity of $R_{\hat{A}}$.

Remark 3.24. Propositon 3.16 allows a unique universal bi-lift of every bicharacter and by Theorems 3.21 and 3.23 right or left quantum group homomorphisms correspond bijectively to bicharacters. Hence right or left quantum group homomorphisms are equivalent to the mutual coactions of $\mathrm{Ng}$ [31. Definition 3.13] and to the special coactions that are considered as morphisms between von Neumann algebraic quantum groups by Kustermans in 22, Propositions 12.1 and 12.4].

Lemma 3.25. Let $\Delta_{L}: A \rightarrow B \otimes A$ and $\Delta_{R}: A \rightarrow A \otimes C$ be a left and a right quantum group homomorphism. Then the following diagram commutes:

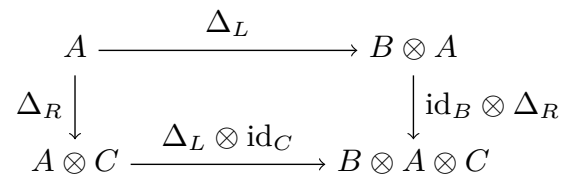

Furthermore, if $B=C$, then $\Delta_{L}$ and $\Delta_{R}$ are associated to the same bicharacter $\chi \in \mathcal{U}(\hat{A} \otimes B)$ if and only if the following diagram commutes:

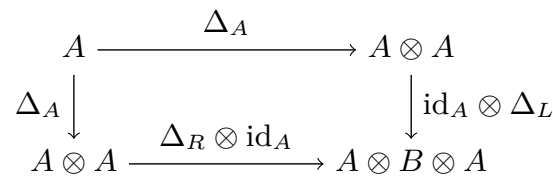

Proof. Since slices of $\mathrm{W}^{A}$ span a dense subspace of $A$, 3.21 commutes if and only if

$$
\left(\operatorname{id}_{\hat{A}} \otimes \operatorname{id}_{B} \otimes \Delta_{R}\right)\left(\operatorname{id}_{\hat{A}} \otimes \Delta_{L}\right)\left(\mathrm{W}^{A}\right)=\left(\operatorname{id}_{\hat{A}} \otimes \Delta_{L} \otimes \operatorname{id}_{C}\right)\left(\operatorname{id}_{\hat{A}} \otimes \Delta_{R}\right)\left(\mathrm{W}^{A}\right) .
$$

Let $\chi$ and $\bar{\chi}$ be the bicharacters associated to $\Delta_{L}$ and $\Delta_{R}$, respectively. Equations 3.15 and 3.19 imply that both sides of $(3.23)$ are equal to $\chi_{12} \mathrm{~W}_{13}^{A} \bar{\chi}_{14}$.

Similarly, the diagram 3.22 commutes if and only if

$$
\left(\operatorname{id}_{\hat{A}} \otimes \operatorname{id}_{A} \otimes \Delta_{L}\right)\left(\operatorname{id}_{\hat{A}} \otimes \Delta_{A}\right)\left(\mathrm{W}^{A}\right)=\left(\operatorname{id}_{\hat{A}} \otimes \Delta_{R} \otimes \operatorname{id}_{A}\right)\left(\operatorname{id}_{\hat{C}} \otimes \Delta_{A}\right)\left(\mathrm{W}^{A}\right)
$$

because the slices on the first leg of $\mathrm{W}^{A}$ span a dense subspace of $A$. Using $(2.8),(3.19)$ and $(3.15)$, we may rewrite (3.24) as $\mathrm{W}_{12}^{A} \bar{\chi}_{13} \mathrm{~W}_{14}^{A}=\mathrm{W}_{12}^{A} \chi_{13} \mathrm{~W}_{14}^{A}$. Thus (3.24) is equivalent to $\bar{\chi}=\bar{\chi}$.

Lemma 3.26. Right or left quantum group homomorphisms are right or left coactions. 
Proof. Equations 3.16 and 3.20 show that left and right quantum group homomorphisms are injective. We only prove the Podleś condition 2.23 for right quantum group homomorphisms, the left case is analogous. Let $\Delta_{R}: A \rightarrow A \otimes B$ be a right quantum group homomorphism with associated bicharacter $V \in \mathcal{U}(\hat{A} \otimes B)$. We must show that the linear span of $\Delta_{R}(A)(1 \otimes B)$ is dense in $A \otimes B$. We may replace $A$ by the dense subspace of slices $\left(\hat{a} \mu \otimes \mathrm{id}_{A}\right) \mathrm{W}^{A}$ for $\mu \in \hat{A}^{\prime}$ and $\hat{a} \in \hat{C}$, where $\hat{a} \mu \in \hat{C}^{\prime}$ is defined by $\hat{a} \mu(x):=\mu(x \hat{a})$ for $\hat{a} \in \hat{A}, \mu \in \hat{A}^{\prime}$, and $x \in \hat{A}$. Given $b \in B$ we have

$$
\left(\left(\hat{a} \mu \otimes \operatorname{id}_{A} \otimes \operatorname{id}_{B}\right)\left(\operatorname{id}_{\hat{A}} \otimes \Delta_{R}\right) \mathrm{W}^{A}\right)(1 \otimes b)=\left(\mu \otimes \operatorname{id}_{A} \otimes \operatorname{id}_{B}\right)\left(\mathrm{W}_{12}^{A} \chi_{13}(\hat{a} \otimes 1 \otimes b)\right) .
$$

Here $\chi_{13}(\hat{a} \otimes 1 \otimes b)$ ranges over a linearly dense subset of $\hat{A} \otimes 1 \otimes B$. Hence we do not change the closed linear span if we replace this expression by $\hat{a} \otimes 1 \otimes b$. This leads to

$$
\left(\mu \otimes \operatorname{id}_{A} \otimes \operatorname{id}_{B}\right)\left(\mathrm{W}_{12}^{A} \cdot(\hat{a} \otimes 1 \otimes b)\right)=\left(\left(\hat{a} \mu \otimes \operatorname{id}_{A}\right) \mathrm{W}^{A}\right) \otimes b,
$$

and these elements span a dense subspace of $A \otimes B$ as asserted.

\subsection{Functors between coaction categories}

Let For: $\mathfrak{C}^{*} \mathfrak{a l g}(\mathbb{G}) \rightarrow \mathfrak{C}^{*} \mathfrak{a l g}$ be the functor that forgets the $\mathbb{G}$-coaction. We now describe quantum group homomorphisms using functors $F: \mathfrak{C}^{*} \mathfrak{a l g}(\mathbb{G}) \rightarrow \mathfrak{C}^{*} \mathfrak{a l g}(\mathbb{H})$ with For $\circ F=$ For. In particular, we show that a right quantum group homomorphism induces such a functor. The results in this section answer a question posed to us by Debashish Goswami.

Theorem 3.27. Let $\mathbb{G}=\left(A, \Delta_{A}\right)$ and $\mathbb{H}=\left(B, \Delta_{B}\right)$ be locally compact quantum groups. Functors $F: \mathfrak{C}^{*} \mathfrak{a l g}(\mathbb{G}) \rightarrow \mathfrak{C}^{*} \mathfrak{a l g}(\mathbb{H})$ with Foro $F=$ For are in natural bijection with right quantum group homomorphisms from $\mathbb{G}$ to $\mathbb{H}$.

More precisely, let $\gamma: D \rightarrow D \otimes A$ be a continuous coaction of $\mathbb{G}$ on a $\mathrm{C}^{*}$-algebra $D$ and let $\Delta_{R}: A \rightarrow$ $A \otimes B$ be a right quantum group homomorphism. Then there is a unique continuous coaction $\alpha$ of $\mathbb{H}$ on $D$ such that the following diagram commutes:

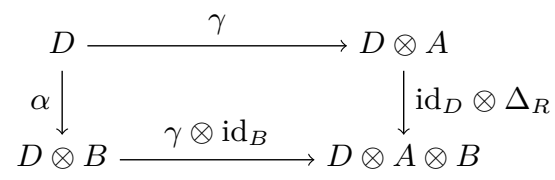

If a morphism $D \rightarrow D^{\prime}$ between two $\mathrm{C}^{*}$-algebras with continuous $\mathbb{G}$-coactions is $\mathbb{G}$-equivariant, then it is $\mathbb{H}$-equivariant as well, so that this construction is a functor $F: \mathfrak{C}^{*} \mathfrak{a l g}(\mathbb{G}) \rightarrow \mathfrak{C}^{*} \mathfrak{a l g}(\mathbb{H})$ with For $\circ=$ For. Conversely, any such functor is of this form for some right quantum group homomorphism $\Delta_{R}$ from $\mathbb{G}$ to $\mathbb{H}$.

Proof. A map $\alpha$ making (3.25) commute is unique if it exists because $\gamma \otimes \mathrm{id}_{B}$ is injective. Existence means $\left(\operatorname{id}_{D} \otimes \Delta_{R}\right) \gamma(D) \subseteq\left(\gamma \otimes \mathrm{id}_{B}\right)(\mathcal{M}(D \otimes B))$. Let $\Delta_{L}: A \rightarrow B \otimes A$ be the left quantum group homomorphism satisfying 3.22). We compute

$$
\begin{aligned}
\left(\operatorname{id}_{D} \otimes \Delta_{R} \otimes \operatorname{id}_{A}\right)\left(\gamma \otimes \operatorname{id}_{A}\right) \gamma & =\left(\operatorname{id}_{D} \otimes \Delta_{R} \otimes \operatorname{id}_{A}\right)\left(\operatorname{id}_{D} \otimes \Delta_{A}\right) \gamma \\
& =\left(\operatorname{id}_{D} \otimes \operatorname{id}_{A} \otimes \Delta_{L}\right)\left(\operatorname{id}_{D} \otimes \Delta_{A}\right) \gamma \\
& =\left(\operatorname{id}_{D} \otimes \operatorname{id}_{A} \otimes \Delta_{L}\right)\left(\gamma \otimes \mathrm{id}_{A}\right) \gamma \\
& =\left(\gamma \otimes \Delta_{L}\right) \gamma=\left(\gamma \otimes \operatorname{id}_{B \otimes A}\right)\left(\mathrm{id}_{D} \otimes \Delta_{L}\right) \gamma
\end{aligned}
$$

where the first and third equality use that $\gamma$ is coassociative 2.22 , the second one uses 3.22 , and the fourth one is trivial.

Thus $\left(\operatorname{id}_{D} \otimes \Delta_{R} \otimes \operatorname{id}_{A}\right)\left(\gamma \otimes \operatorname{id}_{A}\right)$ maps $\gamma(D)$ into $\left(\gamma \otimes \operatorname{id}_{B \otimes A}\right)(\mathcal{M}(D \otimes B \otimes A))$. Since it also maps $1_{D} \otimes A$ into $\left(\gamma \otimes \operatorname{id}_{B \otimes A}\right)(\mathcal{M}(D \otimes B \otimes A))$ and $\gamma(D) \cdot\left(1_{D} \otimes A\right)$ is dense in $D \otimes A$ by the continuity of $\gamma$, $\left(\mathrm{id}_{D} \otimes \Delta_{R} \otimes \mathrm{id}_{A}\right)\left(\gamma \otimes \mathrm{id}_{A}\right)$ maps $D \otimes A$ into $\left(\gamma \otimes \mathrm{id}_{B \otimes A}\right)(\mathcal{M}(D \otimes B \otimes A))$. Thus $\left(\operatorname{id}_{D} \otimes \Delta_{R}\right) \gamma(D) \subseteq$ $\left(\gamma \otimes \operatorname{id}_{B}\right)(\mathcal{M}(D \otimes B))$ as desired. 
The second diagram in (3.14) and several applications of 3.25 imply

$\left(\gamma \otimes \operatorname{id}_{B \otimes B}\right) \circ\left(\alpha \otimes \operatorname{id}_{B}\right) \circ \alpha=\left(\gamma \otimes \operatorname{id}_{B \otimes B}\right) \circ\left(\operatorname{id}_{D} \otimes \Delta_{B}\right) \circ \alpha$.

Since $\gamma \otimes \operatorname{id}_{B \otimes B}$ is injective, $\left(\alpha \otimes \operatorname{id}_{B}\right) \circ \alpha=\left(\operatorname{id}_{D} \otimes \Delta_{B}\right) \circ \alpha$. The map $\alpha$ is injective as well. We check the Podleś condition 2.23 for $\alpha$.

Since $\gamma \otimes \operatorname{id}_{B \otimes A}$ is injective and

$$
\left(\gamma \otimes \Delta_{L}\right) \gamma=\left(\operatorname{id}_{D} \otimes \Delta_{R} \otimes \operatorname{id}_{A}\right)\left(\gamma \otimes \operatorname{id}_{A}\right) \gamma=\left(\gamma \otimes \operatorname{id}_{B \otimes A}\right)\left(\alpha \otimes \operatorname{id}_{A}\right) \gamma
$$

we have $\left(\operatorname{id}_{D} \otimes \Delta_{L}\right) \gamma=\left(\alpha \otimes \operatorname{id}_{A}\right) \gamma$.

Letting $\langle\ldots\rangle$ denote closed linear spans, we first compute

$$
\begin{aligned}
& \left\langle\left(\operatorname{id}_{D} \otimes \Delta_{L}\right) \gamma(d) \cdot\left(1_{D} \otimes b \otimes a\right): a \in A, b \in B \text { and } d \in D\right\rangle \\
& =\left\langle\left(\operatorname{id}_{D} \otimes \Delta_{L}\right)\left(\gamma(d) \cdot\left(1_{D} \otimes \Delta_{L}(a)\right) \cdot\left(1_{D} \otimes b \otimes 1_{A}\right): a \in A, b \in B \text { and } d \in D\right\rangle\right. \\
& =\left\langle\left(\operatorname{id}_{D} \otimes \Delta_{L}\right)\left(\gamma(d) \cdot\left(1_{D} \otimes a\right)\right) \cdot\left(1_{D} \otimes b \otimes 1_{A}\right): a \in A, b \in B \text { and } d \in D\right\rangle \\
& =\left\langle\left(\operatorname{id}_{D} \otimes \Delta_{L}\right)(d \otimes a) \cdot\left(1_{D} \otimes b \otimes 1_{A}\right): a \in A, b \in B \text { and } d \in D\right\rangle \\
& =\left\langle\left(d \otimes\left(\Delta_{L}(a)\left(b \otimes 1_{A}\right)\right)\right): a \in A, b \in B \text { and } d \in D\right\rangle \\
& =D \otimes B \otimes A
\end{aligned}
$$

where the first and fifth equality use the continuity of $\Delta_{L}$ and the third equality uses the Podleś condition for $\gamma$. Hence

$$
\begin{aligned}
& \left\langle(\alpha(d) \otimes a) \cdot\left(1_{D} \otimes b \otimes 1_{A}\right): a \in A, b \in B \text { and } d \in D\right\rangle \\
& =\left\langle\left(\left(\alpha \otimes \operatorname{id}_{A}\right)(d \otimes a)\right) \cdot\left(1_{D} \otimes b \otimes 1_{A}\right): a \in A, b \in B \text { and } d \in D\right\rangle \\
& =\left\langle\left(\left(\alpha \otimes \operatorname{id}_{A}\right)\left(\gamma(d) \cdot\left(1_{D} \otimes a\right)\right) \cdot\left(1_{D} \otimes b \otimes 1_{A}\right): a \in A, b \in B \text { and } d \in D\right\rangle\right. \\
& =\left\langle\left(\left(\alpha \otimes \operatorname{id}_{A}\right) \gamma(d)\right) \cdot\left(1_{D} \otimes b \otimes a\right): a \in A, b \in B \text { and } d \in D\right\rangle \\
& =\left\langle\left(\left(\operatorname{id}_{D} \otimes \Delta_{L}\right) \gamma(d)\right) \cdot\left(1_{D} \otimes b \otimes a\right): a \in A, b \in B \text { and } d \in D\right\rangle \\
& =D \otimes B \otimes A,
\end{aligned}
$$

where the second equality uses the Podles condition of $\gamma$. This implies $\alpha(D) \cdot\left(1_{D} \otimes A\right)=D \otimes A$, that is, $\alpha$ satisfies the Podleś condition.

It is easy to see that a $\mathbb{G}$-equivariant map $D \rightarrow D^{\prime}$ remains $\mathbb{H}$-equivariant for the induced $\mathbb{H}$-coactions. Thus we get a functor $F: \mathfrak{C}^{*} \mathfrak{a l g}(\mathbb{G}) \rightarrow \mathfrak{C}^{*} \mathfrak{a l g}(\mathbb{H})$ with For $\circ F=$ For from a right quantum group homomorphism.

Now let, conversely, $F: \mathfrak{C}^{*} \mathfrak{a l g}(\mathbb{G}) \rightarrow \mathfrak{C}^{*} \mathfrak{a l g}(\mathbb{H})$ be a functor with For $\circ F=$ For, that is, $F$ maps a continuous $\mathbb{G}$-coaction $\gamma: D \rightarrow D \otimes A$ on some $\mathrm{C}^{*}$-algebra $D$ in a natural way to a continuous $\mathbb{H}$-coaction $F(\gamma): D \rightarrow D \otimes B$ on the same $\mathrm{C}^{*}$-algebra. We claim that $F$ must come from some right quantum group homomorphism $\Delta_{R}: A \rightarrow A \otimes B$ by the above construction.

When we apply $F$ to the coaction $\Delta_{A}: A \rightarrow A \otimes A$, we get an $\mathbb{H}$-coaction $\Delta_{R}: A \rightarrow A \otimes B$. Being a coaction, it makes the second diagram in 3.14 commute. We will see later that the first diagram in (3.14) also commutes. First we use naturality to show that 3.25 with $\alpha=F(\gamma)$ commutes for any coaction of $\mathbb{G}$, so that $\Delta_{R}$ determines the functor $F$.

To begin with, we consider the coaction $\Delta_{A} \oplus \Delta_{A}: A \oplus A \rightarrow(A \oplus A) \otimes A$. Since the coordinate projections $\pi_{1}, \pi_{2}: A \oplus A \rightarrow A$ are $\mathbb{G}$-equivariant, they are $\mathbb{H}$-equivariant with respect to $F\left(\Delta_{A} \oplus \Delta_{A}\right)$ and $F\left(\Delta_{A}\right)=\Delta_{R}$. This already implies that $F\left(\Delta_{A} \oplus \Delta_{A}\right)=\Delta_{R} \oplus \Delta_{R}$.

Next we consider the coaction $\operatorname{id}_{\mathbb{K}(\mathcal{H})} \otimes \Delta_{B}$ on $\mathbb{K}(\mathcal{H}) \otimes A$. For any projection $P \in \mathbb{K}(\mathcal{H})$, we get a $\mathbb{G}$-equivariant morphism

$$
A \oplus A \rightarrow \mathbb{K}(\mathcal{H}) \otimes A, \quad(a, b) \mapsto P \otimes a+(1-P) \otimes b .
$$

Since we already know the $\mathbb{H}$-coaction $F\left(\Delta_{A} \oplus \Delta_{A}\right)$, the induced $\mathbb{H}$-coaction on $\mathbb{K}(\mathcal{H}) \otimes A$ maps $P \otimes a \mapsto$ $P \otimes \Delta_{R}(a)$. Since this holds for all projections $P$ and since these projections generate $\mathbb{K}(\mathcal{H})$, we get $F\left(\operatorname{id}_{\mathbb{K}(\mathcal{H})} \otimes \Delta_{A}\right)=\operatorname{id}_{\mathbb{K}(\mathcal{H})} \otimes \Delta_{R}$. 
Now consider a general coaction $\gamma: D \rightarrow D \otimes A$. Then $\gamma$ is $\mathbb{G}$-equivariant with respect to the coaction $\operatorname{id}_{D} \otimes \Delta_{A}$ on $D \otimes A$. Let $\pi: D \rightarrow \mathbb{K}(\mathcal{H})$ be a morphism coming from a faithful representation of $D$ on some Hilbert space $\mathcal{H}$. The injective, $\mathbb{G}$-equivariant morphism $\left(\pi \otimes \operatorname{id}_{A}\right) \circ \gamma: D \rightarrow \mathbb{K}(\mathcal{H}) \otimes A$ remains $\mathbb{H}$-equivariant with respect to the coactions $F(\gamma)$ on $A$ and $F\left(\operatorname{id}_{\mathbb{K}(\mathcal{H})} \otimes \Delta_{A}\right)=\operatorname{id}_{\mathbb{K}(\mathcal{H})} \otimes \Delta_{R}$ on $\mathbb{K}(\mathcal{H}) \otimes A$. This means that 3.25) commutes with $\alpha:=F(\gamma)$. Finally, specialising 3.25 to the coaction $\Delta_{A}$ on $A$ shows that the first diagram in (3.14) commutes. Thus $\Delta_{R}$ is a right quantum group homomorphism that generates $F$. The construction also shows that $\Delta_{R}$ is unique.

Let $\mathbb{I}=\left(C, \Delta_{C}\right)$ be a quantum group.

Proposition 3.28. Let $\alpha: A \rightarrow A \otimes B$ and $\beta: B \rightarrow B \otimes C$ be two right quantum group homomorphisms and let $F_{\alpha}: \mathfrak{C}^{*} \mathfrak{a l g}(\mathbb{G}) \rightarrow \mathfrak{C}^{*} \mathfrak{a l g}(\mathbb{H})$ and $F_{\beta}: \mathfrak{C}^{*} \mathfrak{a l g}(\mathbb{H}) \rightarrow \mathfrak{C}^{*} \mathfrak{a l g}(\mathbb{I})$ be the associated functors. Then $F_{\beta} \circ F_{\alpha}=F_{\gamma}$, where $\gamma: A \rightarrow A \otimes C$ is the unique right quantum group homomorphism that makes the following diagram commute:

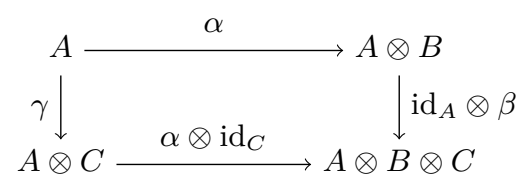

Furthermore, the bicharacter associated to $\gamma$ is the composite of the bicharacters associated to $\beta$ and $\alpha$.

Proof. Theorem 3.27 shows that $F_{\alpha}$ maps the coaction $\Delta_{A}$ to $\alpha$. This is mapped by $F_{\beta}$ to the unique morphism making (3.26) commute. Thus $F_{\beta} \circ F_{\alpha}$ maps $\Delta_{A}$ to $\gamma$, forcing $F_{\beta} \circ F_{\alpha}=F_{\gamma}$.

Theorem 3.27 yields a unique continuous right coaction $\gamma$ of $\mathbb{I}$ on $A$ making 3.26 commute. It is not hard to show that this is a right quantum group homomorphism. Anyway, we want to convince ourselves that this construction corresponds to the composition of bicharacters.

Since slices of $\mathrm{W}^{A}$ by continuous linear functionals on $\hat{A}$ generate a dense subspace of $A$, the diagram (3.26) commutes if and only if

$$
\left(\mathrm{id}_{\hat{A}} \otimes \mathrm{id}_{A} \otimes \beta\right)\left(\mathrm{id}_{\hat{A}} \otimes \alpha\right)\left(\mathrm{W}^{A}\right)=\left(\mathrm{id}_{\hat{A}} \otimes \alpha \otimes \mathrm{id}_{C}\right)\left(\mathrm{id}_{\hat{A}} \otimes \gamma\right)\left(\mathrm{W}^{A}\right) .
$$

Equation (3.15) implies $\left(\mathrm{id}_{\hat{A}} \otimes \alpha\right) \mathrm{W}^{A}=\mathrm{W}_{12}^{A} \chi_{13}^{A \rightarrow B}$, and $\operatorname{id}_{\hat{C}} \otimes \mathrm{id}_{A} \otimes \beta$ maps this to the element represented by the unitary operator

$$
\mathbb{W}_{12}^{A} \chi_{34}^{B \rightarrow C} \chi_{13}^{A \rightarrow B}\left(\chi_{34}^{B \rightarrow C}\right)^{*}=\mathbb{W}_{12}^{A} \chi_{13}^{A \rightarrow B} \chi_{14}^{A \rightarrow C}
$$

by 3.16 and 3.5 . Thus

$$
\left(\mathrm{id}_{\hat{A}} \otimes \mathrm{id}_{A} \otimes \beta\right)\left(\mathrm{id}_{\hat{A}} \otimes \alpha\right)\left(\mathrm{W}^{A}\right)=\mathrm{W}_{12}^{A} \chi_{13}^{A \rightarrow B} \chi_{14}^{A \rightarrow C},
$$

where $\chi^{A \rightarrow C}:=\chi^{B \rightarrow C} * \chi^{B \rightarrow C}$. Let $\bar{\chi}$ be the bicharacter associated to $\gamma$. Equation 3.15 implies

$$
\left(\operatorname{id}_{\hat{A}} \otimes \alpha \otimes \operatorname{id}_{C}\right)\left(\operatorname{id}_{\hat{A}} \otimes \gamma\right)\left(\mathrm{W}^{A}\right)=\left(\operatorname{id}_{\hat{A}} \otimes \alpha \otimes \operatorname{id}_{C}\right)\left(\mathrm{W}_{12}^{A} \bar{\chi}_{13}\right)=\mathrm{W}_{12}^{A} \chi_{13}^{A \rightarrow B} \bar{\chi}_{14} .
$$

Hence 3.26 commutes if and only if $\bar{\chi}=\chi^{A \rightarrow C}$.

Example 3.29. Let $\chi^{A \rightarrow B} \in \mathcal{U}(\hat{A} \otimes B)$ and $\chi^{B \rightarrow C} \in \mathcal{U}(\hat{B} \otimes C)$ be bicharacters.

Assume first that $\chi^{B \rightarrow C}$ comes from a Hopf *-homomorphism $f: B \rightarrow C$, that is, $\chi^{B \rightarrow C}=\left(\operatorname{id}_{\hat{B}} \otimes f\right)\left(\mathrm{W}^{B}\right)$. Let $\alpha$ be the right quantum group homomorphism from $\mathbb{G}$ to $\mathbb{H}$ associated to $\chi^{A \rightarrow B}$. The right quantum group homomorphism from $\mathbb{H}$ to $\mathbb{I}$ associated to $\chi^{B \rightarrow C}$ is $\beta:=\left(\operatorname{id}_{A} \otimes f\right) \Delta_{B}$. The following computation shows that $\gamma=\left(\mathrm{id}_{A} \otimes f\right) \alpha$ satisfies 3.26 :

$$
\begin{aligned}
& \left(\mathrm{id}_{\hat{A}} \otimes \mathrm{id}_{A} \otimes \beta\right)\left(\mathrm{id}_{\hat{A}} \otimes \alpha\right) \mathrm{W}^{A} \\
& =\left(\operatorname{id}_{\hat{A}} \otimes \mathrm{id}_{A} \otimes \mathrm{id}_{B} \otimes f\right)\left(\mathrm{id}_{\hat{A}} \otimes \mathrm{id}_{A} \otimes \Delta_{B}\right) \mathrm{W}_{12}^{A} \chi_{13}^{A \rightarrow B} \\
& =\left(\mathrm{id}_{\hat{A}} \otimes \mathrm{id}_{A} \otimes \mathrm{id}_{B} \otimes f\right) \mathrm{W}_{12}^{A} \chi_{13}^{A \rightarrow B} \chi_{14}^{A \rightarrow B} \\
& =\left(\operatorname{id}_{\hat{A}} \otimes \operatorname{id}_{A} \otimes \operatorname{id}_{B} \otimes f\right)\left(\operatorname{id}_{\hat{A}} \otimes \alpha \otimes \mathrm{id}_{B}\right) \mathrm{W}_{12}^{A} \chi_{13}^{A \rightarrow B} \\
& =\left(\operatorname{id}_{\hat{A}} \otimes \alpha \otimes \operatorname{id}_{C}\right)\left(\operatorname{id}_{\hat{A}} \otimes\left(\operatorname{id}_{A} \otimes f\right) \alpha\right) \mathrm{W}^{A} ;
\end{aligned}
$$

the first step uses $(3.15)$; the second step uses $(3.2)$; the third and the last step use 3.15 . Proposition 3.28 yields $\beta * \alpha=\left(\operatorname{id}_{A} \otimes f\right) \alpha$. Hence the composite $\chi^{B \rightarrow C} * \chi^{A \rightarrow B}$ is $\left(\operatorname{id}_{A} \otimes f\right) \chi^{A \rightarrow B}$. 
Example 3.30. Let $\chi^{A \rightarrow B}$ be constructed from a Hopf ${ }^{*}$-homomorphism $f: \hat{B} \rightarrow \hat{A}$, that is, $\chi^{A \rightarrow B}=$ $\left(f \otimes \operatorname{id}_{B}\right)\left(\mathrm{W}^{B}\right)$. Then the composite $\chi^{A \rightarrow C}$ is $\left(f \otimes \operatorname{id}_{C}\right)\left(\chi^{B \rightarrow C}\right)$. This follows from Example 3.29 because $A \mapsto \hat{A}$ is a contravariant functor on bicharacters.

Proposition 3.31. A right quantum group homomorphism from $\mathbb{G}$ to $\mathbb{H}$ induces a natural map from Hilbert space corepresentations of $\mathbb{G}$ to corepresentations of $\mathbb{H}$ on the same Hilbert space.

Proof. Given any corepresentation $\mathrm{U}^{A} \in \mathcal{U}(\mathbb{K}(\mathcal{H}) \otimes A)$ we can use 3.16 in this more general framework: replacing $\mathrm{W}^{A}$ by $\mathrm{U}^{A}$ we get a unique unitary $\mathrm{U}^{B} \in \mathcal{U}(\mathbb{K}(\mathcal{H}) \otimes B)$ such that

$$
\left(\mathrm{id} \otimes \Delta_{R}\right) \mathrm{U}^{A}=\mathrm{U}_{12}^{A} \mathrm{U}_{13}^{B} \quad \text { in } \mathcal{U}(\mathbb{K}(\mathcal{H}) \otimes A \otimes B) .
$$

The computation in the proof of Theorem 3.21 showing that $\chi$ satisfies the character condition on the second leg yields that $\mathrm{U}^{B} \in \mathcal{U}(\mathbb{K}(\mathcal{H}) \otimes B)$ is a corepresentation of $\mathbb{H}$ on $\mathcal{H}$.

\subsection{Comparison with group homomorphisms}

Let $G$ and $H$ be locally compact groups. Hopf *-homomorphisms from $\mathrm{C}_{0}(H)$ to $\mathrm{C}_{0}(G)$ are equivalent to continuous group homomorphisms $G \rightarrow H$. By Theorem 3.17, it follows that bicharacters must also correspond to classical group homomorphisms. And the same holds for right and left quantum group homomorphisms. We are going to establish this directly.

Let $\varphi: G \rightarrow H$ be a continuous group homomorphism. Then $V_{\varphi}(g):=\lambda_{\varphi(g)}$ defines a bicharacter in $\mathcal{U}\left(\mathrm{C}_{0}(G) \otimes \mathrm{C}_{\mathrm{r}}^{*}(H)\right)$, that is, a quantum group homomorphism from $\mathrm{C}_{\mathrm{r}}^{*}(G)$ to $\mathrm{C}_{\mathrm{r}}^{*}(H)$.

Lemma 3.32. Let $G$ and $H$ be locally compact groups. Then every bicharacter from $\left(\mathrm{C}_{\mathrm{r}}^{*}(G), \Delta_{\mathrm{C}_{\mathrm{r}}^{*}(G)}\right)$ to $\left(\mathrm{C}_{\mathrm{r}}^{*}(H), \Delta_{\mathrm{C}_{\mathrm{r}}^{*}(H)}\right)$ is induced by a unique continuous group homomorphism $\varphi: G \rightarrow H$ as above.

Proof. It is clear that the bicharacter $\chi_{\varphi}$ determines $\varphi$. Thus it remains to observe that every bicharacter $\chi$ in $\mathcal{U}\left(\mathrm{C}_{0}(G) \otimes \mathrm{C}_{\mathrm{r}}^{*}(H)\right)$ is of this form for a continuous group homomorphism $\varphi: G \rightarrow H$. We may view $\chi$ as a strictly continuous function from $G$ to $\mathcal{U}\left(\mathrm{C}_{\mathrm{r}}^{*}(H)\right)$. Equation 3.2 means that its values are grouplike elements of $\mathcal{U}\left(\mathrm{C}_{\mathrm{r}}^{*}(H)\right)$ for each $g \in G$, that is, $\Delta_{\mathrm{C}_{\mathrm{r}}^{*}(H)}(\chi(g))=\chi(g) \otimes \chi(g)$. This implies $\chi(g)=\lambda_{\varphi(g)}$ for some $\varphi(g) \in H$. The map $\varphi: G \rightarrow H$ must be continuous in order for $g \mapsto \lambda_{\varphi(g)}$ to be strictly continuous. Finally, 3.1 means that the map $\varphi$ is a group homomorphism.

Example 3.33. Let $A=\mathrm{C}_{0}(G)$ and $B=\mathrm{C}_{0}(H)$ for locally compact groups $G$ and $H$. A right quantum group homomorphism from $A$ to $A \otimes B$ corresponds to a continuous map $\alpha: G \times H \rightarrow G$, which we denote by $\alpha(g, h):=g \cdot h$. The commutativity of the first diagram in (3.14) means that $\left(g_{1} \cdot g_{2}\right) \cdot h=g_{1} \cdot\left(g_{2} \cdot h\right)$ for all $g_{1}, g_{2} \in G, h \in H$, so that $g \cdot h=g \cdot \varphi(h)$ for all $g \in G, h \in H$ for a continuous map $\varphi: H \rightarrow G$ defined by $\varphi(h):=1 \cdot h$. The commutativity of the second diagram (3.14) is equivalent to $\varphi\left(h_{1} \cdot h_{2}\right)=\varphi\left(h_{1}\right) \cdot \varphi\left(h_{2}\right)$ for all $h_{1}, h_{2} \in H$. Thus right quantum group homomorphisms $\mathrm{C}_{0}(G) \rightarrow \mathrm{C}_{0}(H)$ correspond to continuous group homomorphisms $H \rightarrow G$.

Example 3.34. Let $A=\mathrm{C}_{\mathrm{r}}^{*}(G)$ and $B=\mathrm{C}_{\mathrm{r}}^{*}(H)$ for second countable locally compact groups $G$ and $H$. We claim that right quantum group homomorphisms from $\mathrm{C}_{\mathrm{r}}^{*}(G)$ to $\mathrm{C}_{\mathrm{r}}^{*}(H)$ correspond bijectively to continuous group homomorphisms $G \rightarrow H$.

Since $A \otimes B=\mathrm{C}_{\mathrm{r}}^{*}(G \times H)$, a morphism from $A$ to $A \otimes B$ must come from a continuous representation $g \mapsto u_{g}$ of $G$ by unitary multipliers of $\mathrm{C}_{\mathrm{r}}^{*}(G \times H)$. To be a right quantum group homomorphism, it suffices to check that the diagrams in (3.14) commute on the unitary multipliers $\delta_{g}$ for $g \in G$. The commutativity of the first diagram in (3.14 becomes $\left(\Delta_{A} \otimes \mathrm{id}_{B}\right)\left(u_{g}\right)=\delta_{g} \otimes u_{g}$, forcing $u_{g}=\delta_{g} \cdot u_{g}^{\prime}$ for unitary multipliers $u_{g}^{\prime}$ of $B$. The commutativity of the second diagram in 3.14 becomes $\Delta_{B}\left(u_{g}^{\prime}\right)=u_{g}^{\prime} \otimes u_{g}^{\prime}$, forcing $u_{g}^{\prime}=\delta_{\varphi(g)}$ for some $\varphi(g) \in H$. The map $g \mapsto \varphi(g)$ is a measurable group homomorphism. In the separable case, measurability implies continuity, so that $\varphi$ is a continuous group homomorphism $G \rightarrow H$. 


\subsection{Projections on quantum groups}

Projections on quantum groups will be used later in Chapter 6 Let $\mathbb{I}=\left(C, \Delta_{C}\right)$ be a quantum group and let $\mathrm{W}^{C} \in \mathcal{U}(\hat{C} \otimes C)$ be the reduced bicharacter. Let $(\pi, \hat{\pi})$ and $(\rho, \hat{\rho})$ be $\mathbb{I}$-Heisenberg and -anti Heisenberg pairs on $\mathcal{H}_{\pi}$ and $\mathcal{H}_{\rho}$, respectively.

Definition 3.35. A bicharacter $\mathrm{P} \in \mathcal{U}(\hat{C} \otimes C)$ is a projection if $\mathrm{P} * \mathrm{P}=\mathrm{P}$, where $*$ denotes the composition of bicharacters in Definition 3.6. Equivalently, $\mathrm{P} \in \mathcal{U}(\hat{C} \otimes C)$ satisfies either of the following relations:

$$
\begin{array}{cc}
\mathrm{P}_{\hat{\pi} 3} \mathrm{P}_{1 \pi}=\mathrm{P}_{1 \pi} \mathrm{P}_{13} \mathrm{P}_{\hat{\pi} 3} & \text { in } \mathcal{U}\left(\hat{C} \otimes \mathbb{K}\left(\mathcal{H}_{\pi}\right) \otimes C\right), \\
\mathrm{P}_{1 \rho} \mathrm{P}_{\hat{\rho} 3}=\mathrm{P}_{\hat{\rho} 3} \mathrm{P}_{13} \mathrm{P}_{1 \rho} \quad \text { in } \mathcal{U}\left(\hat{C} \otimes \mathbb{K}\left(\mathcal{H}_{\rho}\right) \otimes C\right) .
\end{array}
$$

Therefore, a projection, while viewed as an operator acting on $\mathcal{H}_{\pi} \otimes \mathcal{H}_{\pi}$, satisfies the pentagon equation 2.4. Equivalently, the operator $\mathbb{P}:=(\hat{\pi} \otimes \pi) \mathrm{P} \in \mathcal{U}\left(\mathcal{H}_{\pi} \otimes \mathcal{H}_{\pi}\right)$ is a multiplicative unitary. Hence the dual of a projection is also a projection. Manageability of bicharacters immediately leads to the following proposition:

Proposition 3.36. $\mathbb{P} \in \mathcal{U}\left(\mathcal{H}_{\pi} \otimes \mathcal{H}_{\pi}\right)$ is a manageable multiplicative unitary.

Proof. Without loss of generality, assume that $\mathbb{W}^{C}=(\hat{\pi} \otimes \pi) \in \mathcal{U}\left(\mathcal{H}_{\pi} \otimes \mathcal{H}_{\pi}\right)$ is a manageable multiplicative unitary. By Corollary 3.11 $\mathbb{P}$ commutes with $Q_{C} \otimes Q_{C}$, where $Q_{C}$ is the positive, self-adjoint operator with trivial kernel defining manageability of $\mathbb{W}^{C}$. Finally, Equation 3.12 gives $\widetilde{\mathbb{P}} \in \mathcal{U}\left(\overline{\mathcal{H}}_{\pi} \otimes \mathcal{H}_{\pi}\right)$ satisfying the manageability condition:

$$
(x \otimes u|\mathbb{P}| z \otimes y)=\left(\bar{z} \otimes Q_{C} u|\widetilde{\mathbb{P}}| \bar{x} \otimes Q_{C}^{-1} y\right),
$$

for all $x, z \in \mathcal{H}_{A}, u \in \mathcal{D} \operatorname{om}\left(Q_{C}\right)$ and $y \in \mathcal{D o m}\left(Q_{C}^{-1}\right)$.

Definition 3.37. The quantum group $\mathbb{G}=\left(A, \Delta_{A}\right)$ generated by $\mathbb{P} \in \mathcal{U}\left(\mathcal{H}_{\pi} \otimes \mathcal{H}_{\pi}\right)$ is called the image of the projection $\mathrm{P} \in \mathcal{U}(\hat{C} \otimes C)$. Moreover, $\mathrm{W}^{A}=\mathrm{P} \in \mathcal{U}(\hat{A} \otimes A)$ is the reduced bicharacter of $\mathbb{G}$.

Identification of $\mathrm{P}$ with $\mathrm{W}^{A}$ gives the following bicharacter conditions:

$$
\begin{array}{ll}
\left(\hat{\Delta}_{A} \otimes \mathrm{id}_{A}\right) \mathrm{P}=\mathrm{P}_{23} \mathrm{P}_{13} & \text { in } \mathcal{U}(\hat{A} \otimes \hat{A} \otimes A), \\
\left(\operatorname{id}_{\hat{A}} \otimes \Delta_{A}\right) \mathrm{P}=\mathrm{P}_{12} \mathrm{P}_{13} & \text { in } \mathcal{U}(\hat{A} \otimes A \otimes A) .
\end{array}
$$

The dual of $\mathbb{P} \in \mathcal{U}\left(\mathcal{H}_{\pi} \otimes \mathcal{H}_{\pi}\right)$ defined by $\widehat{\mathbb{P}}:=\Sigma \mathbb{P}^{*} \Sigma \in \mathcal{U}\left(\mathcal{H}_{\pi} \otimes \mathcal{H}_{\pi}\right)$ is also a manageable multiplicative unitary. Therefore, $\widehat{\mathbb{P}}$ generates $\widehat{\mathbb{G}}=\left(\hat{A}, \hat{\Delta}_{A}\right)$ and $\widehat{\mathrm{W}}^{A}=\widehat{\mathrm{P}} \in \mathcal{U}(A \otimes \hat{A})$.

Example 3.38. In particular, if $\hat{C}=\mathrm{C}_{0}(G)$ for a group $G$, then by Lemma 3.32 any projection $\mathrm{P} \in$ $\mathcal{U}\left(\mathrm{C}_{0}(G) \otimes \mathrm{C}_{\mathrm{r}}^{*}(G)\right)$ corresponds to an idempotent group homomorphism $p: G \rightarrow G$ and $\hat{A}=\mathrm{C}_{0}(p(G))$.

Let $A, B$ be $\mathrm{C}^{*}$-algebras and $T \in \mathcal{U}(A \otimes B)$. Then $B$ is generated by $T$ in the sense of [46. Definition 4.1], if for any representation $\xi: B \rightarrow \mathbb{B}(\mathcal{H})$ and any $\mathrm{C}^{*}$-algebra $C \subset \mathbb{B}(\mathcal{H})$ the condition $\left(\mathrm{id}_{A} \otimes \xi\right) T \in \mathcal{M}(A \otimes C)$ implies that $\xi \in \operatorname{Mor}(B, C)$. In 39, Section 3.5] it is shown that every corepresentation $\mathrm{U} \in \mathcal{U}(\mathbb{K}(\mathcal{H}) \otimes A)$ of $\mathbb{G}=\left(A, \Delta_{A}\right)$ on a Hilbert space $\mathcal{H}$ gives rise to a nondegenerate $\mathrm{C}^{*}$-subalgebra $D_{\mathrm{U}}=\{(\mathrm{id} \otimes \omega) \mathrm{U}$ : $\left.\omega \in A^{\prime}\right\}^{\mathrm{CLS}}$ of $\mathbb{B}(\mathcal{H})$. By $[9$, Proposition 2.1] a corepresentation $\mathrm{U} \in \mathcal{U}(\mathbb{K}(\mathcal{H}) \otimes A)$ of $\mathbb{G}$ generates a nondegenerate $\mathrm{C}^{*}$-algebra $D$ of $\mathbb{B}(\mathcal{H})$ if and only if $D=D_{\mathrm{U}}$.

Definition 3.39 (9, Definition 3.2]). Let $\mathbb{I}=\left(C, \Delta_{C}\right), \mathbb{G}=\left(A, \Delta_{A}\right)$ be quantum groups. $\mathbb{G}$ is called a closed quantum subgroup of $\mathbb{I}$ in the sense of Woronowicz if there is a bicharacter $\chi \in \mathcal{U}(\hat{C} \otimes A)$ that generates $\mathbb{G}$.

Therefore, $\mathbb{G}$ in the Definition 3.39 is a closed quantum subgroup of $\mathbb{I}$ in the sense of Woronowicz. By duality, we get $\widehat{\mathbb{G}}$ as a closed quantum subgroup of $\widehat{\mathbb{I}}$ in the same sense. Let $\Delta_{R}: C \rightarrow C \otimes C$ and $\Delta_{L}: C \rightarrow C \otimes C$ be the right and left quantum group homomorphism constructed out of the bicharacter $\mathrm{P} \in \mathcal{U}(\hat{C} \otimes C)$. 
Lemma 3.40. Given any projection $\mathrm{P} \in \mathcal{U}(\hat{C} \otimes C)$ we have

$$
\begin{aligned}
& \left.\Delta_{C}\right|_{A}=\left.\Delta_{L}\right|_{A}=\left.\Delta_{R}\right|_{A}=\Delta_{A}, \\
& \left.\hat{\Delta}_{C}\right|_{\hat{A}}=\left.\hat{\Delta}_{L}\right|_{\hat{A}}=\left.\hat{\Delta}_{R}\right|_{\hat{A}}=\hat{\Delta}_{A} .
\end{aligned}
$$

Proof. It sufficies to show (3.32). Then $(3.33)$ follows by duality.

Since $\left\{\left(\omega \otimes \mathrm{id}_{A}\right) \mathrm{P}: \omega \in \bar{A}^{\prime}\right\}$ is dense in $A \subseteq \mathcal{M}(C)$ and $\hat{A} \subseteq \mathcal{M}(\hat{C})$ for any $a=\left(\omega \otimes \operatorname{id}_{A}\right) \mathrm{P} \in A \subseteq \mathcal{M}(C)$ we have

$$
\Delta_{C}(a)=\left(\omega \otimes \operatorname{id}_{C} \otimes \operatorname{id}_{C}\right)\left(\operatorname{id}_{\hat{C}} \otimes \Delta_{C}\right) \mathrm{P}=\left(\omega \otimes \operatorname{id}_{A} \otimes \operatorname{id}_{A}\right) \mathrm{P}_{12} \mathrm{P}_{13}=\left(\omega \otimes \Delta_{A}\right) \mathrm{P}=\Delta_{A}(a)
$$

where the second equality uses $(3.2)$ and the third equality uses 3.31 .

Let $(\rho, \hat{\rho})$ be an $\mathbb{I}$-anti-Heisenberg pair on $\mathcal{H}_{\rho}$. We rewrite 3.3$)$ for $\mathrm{P}$ as:

$$
\mathrm{W}_{1 \rho}^{C} \mathrm{P}_{\hat{\rho} 3}=\mathrm{P}_{\hat{\rho} 3} \mathrm{P}_{13} \mathrm{~W}_{1 \rho}^{C} \quad \text { in } \mathcal{U}\left(\hat{C} \otimes \mathbb{K}\left(\mathcal{H}_{\rho}\right) \otimes A\right) .
$$

Now 3.19 gives

$$
\left(\operatorname{id}_{C} \otimes \rho\right) \Delta_{L}(c)=\widehat{\mathrm{P}}_{1 \hat{\rho}}\left(1_{C} \otimes \rho(c)\right) \widehat{\mathrm{P}}_{1 \hat{\rho}}^{*} .
$$

For $a=\left(\omega \otimes \operatorname{id}_{A}\right) \mathrm{P} \in A$ we compute:

$$
\begin{aligned}
&\left(\operatorname{id}_{\hat{C}} \otimes \rho\right) \Delta_{L}(a)=\widehat{\mathrm{P}}_{1 \hat{\rho}}\left(1_{C} \otimes \rho(a)\right) \widehat{\mathrm{P}}_{1 \hat{\rho}}^{*}=\left(\omega \otimes \operatorname{id}_{C} \otimes \operatorname{id}_{\mathcal{H}_{\rho}}\right) \widehat{\mathrm{P}}_{2 \hat{\rho}} \mathrm{P}_{1 \rho} \widehat{\mathrm{P}}_{2 \hat{\rho}}^{*} \\
&=\left(\omega \otimes \operatorname{id}_{A} \otimes \rho\right) \mathrm{P}_{12} \mathrm{P}_{13}=\left(\omega \otimes\left(\operatorname{id}_{A} \otimes \rho\right) \Delta_{A}\right) \mathrm{P}=\left(\operatorname{id}_{C} \otimes \rho\right) \Delta_{A}(a) .
\end{aligned}
$$

The first equality uses (3.34), the third equality uses (3.29), and the fourth equality uses (3.31).

Similarly, given any $\mathbb{I}$-Heisenberg pair $(\pi, \hat{\pi})$ we can show

$$
\left(\pi \otimes \operatorname{id}_{C}\right) \Delta_{R}(a)=\left(\pi \otimes \operatorname{id}_{A}\right) \Delta_{A}(a) \quad \text { for all } a \in A .
$$

Finally, the faithfulness of $\pi$ and $\rho$ yields 3.32 .

Notation 3.41. Let $\mathfrak{C}^{*} \mathcal{Q} \mathfrak{G} \mathfrak{p}$ denote the category where objects are $\mathrm{C}^{*}$-quantum groups and bicharacters are arrows.

Every projection bicharacter $\mathrm{P} \in \mathcal{U}(\hat{C} \otimes C)$ is an idempotent arrow on $\mathbb{I}$ in $\mathfrak{C}^{*} \mathcal{Q} \mathfrak{G} \mathfrak{r}$ with the image $\mathbb{G}$. Hence $\mathrm{P}$ is a split idempotent. We make this precise in the following lemma:

Lemma 3.42. Let $\mathrm{P} \in \mathcal{U}(\hat{C} \otimes C)$ be a projection bicharacter and let $\mathbb{G}=\left(A, \Delta_{A}\right)$ be its image. Then there are bicharacters $\chi^{C \rightarrow A} \in \mathcal{U}(\hat{C} \otimes A)$ and $\chi^{A \rightarrow C} \in \mathcal{U}(\hat{A} \otimes C)$ such that

$$
\mathrm{P}=\chi^{A \rightarrow C} * \chi^{C \rightarrow A} \quad \text { and } \quad \chi^{C \rightarrow A} * \chi^{A \rightarrow C}=\mathrm{W}^{A} .
$$

Proof. Let $\mathrm{P} \in \mathcal{U}(\hat{C} \otimes C)$ be a projection with the image $\mathbb{G}=\left(A, \Delta_{A}\right)$. Then $\mathrm{P} \in \mathcal{U}(\hat{A} \otimes A) \subset \mathcal{U}(\hat{C} \otimes C)$. This gives the Hopf ${ }^{*}$-homomorphisms $\varphi: A \rightarrow C$ and $\psi: \hat{A} \rightarrow \hat{C}$. By Example 3.4 we construct the following bicharacters

$$
\begin{array}{ll}
\chi^{C \rightarrow A}:=\left(\psi \otimes \mathrm{id}_{A}\right) \mathrm{W}^{A}=\mathrm{P} & \text { in } \mathcal{U}(\hat{C} \otimes A), \\
\chi^{A \rightarrow C}:=\left(\operatorname{id}_{\hat{A}} \otimes \varphi\right) \mathrm{W}^{A}=\mathrm{P} & \text { in } \mathcal{U}(\hat{A} \otimes C) .
\end{array}
$$

The pentagon equation 3.28 yields 3.35 . 


\section{Chapter 4}

\section{Twisted tensor products of $\mathrm{C}^{*}$-algebras}

Several important constructions put together two $\mathrm{C}^{*}$-algebras in a kind of tensor product where the tensor factors do not commute. For instance, a noncommutative two-torus is obtained in this way from two copies of $\mathrm{C}(\mathbb{T})$. More generally, the crossed product $A \rtimes_{\alpha} G$ for a continuous action $\alpha: G \rightarrow \operatorname{Aut}(A)$ of a locally compact group $G$ combines $A$ and the group $\mathrm{C}^{*}$-algebra of $G$. Such crossed products also exist for locally compact quantum groups. Another example is the graded tensor product for $\mathbb{Z} / 2$-graded $\mathrm{C}^{*}$-algebras, which is defined so that the odd elements anticommute.

Definition 4.1 (compare [4] ). Let $A, B, C$ be $\mathrm{C}^{*}$-algebras, $\alpha \in \operatorname{Mor}(A, C)$ and $\beta \in \operatorname{Mor}(B, C)$. If $\alpha(A) \cdot \beta(B)=C$, then we call $(C, \alpha, \beta)$ a crossed product or crossed tensor product of $A$ and $B$.

Example 4.2. The spatial tensor product $C=A \otimes B$ of two $\mathrm{C}^{*}$-algebras with $\alpha(a)=a \otimes 1_{B}$ and $\beta(b)=1_{A} \otimes b$ is the simplest example of a crossed product.

Let $\alpha$ and $\beta$ be (nondegenerate) representations of $A$ and $B$ on the same Hilbert space $\mathcal{H}$ such that $\alpha(A) \cdot \beta(B)$ and $\beta(B) \cdot \alpha(A)$ are the same subspace of $\mathbb{B}(\mathcal{H})$. Then $C:=\alpha(A) \cdot \beta(B)$ is a $\mathrm{C}^{*}$-algebra, $\alpha \in \operatorname{Mor}(A, C)$ and $\beta \in \operatorname{Mor}(B, C)$. Thus $C$ is a crossed product of $A$ and $B$. This suggests that crossed products are defined by some commutation relations between $\alpha$ and $\beta$. In a purely algebraic setting, Exel 13 describes the multiplication on $C$ through the map $\tau: B \otimes A \rightarrow A \otimes B$ expressing $\beta(b) \alpha(a)$ as a linear combination of elements of the form $\alpha\left(a^{\prime}\right) \beta\left(b^{\prime}\right)$; but this does not work for $\mathrm{C}^{*}$-algebras because of the completions involved.

Definition 4.3. Two crossed products $C_{1}=\alpha_{1}(A) \cdot \beta_{1}(B)$ and $C_{2}=\alpha_{2}(A) \cdot \beta_{2}(B)$ of $A$ and $B$ are equivalent if there is a faithful morphism $\varphi \in \operatorname{Mor}\left(C_{1}, C_{2}\right)$ with $\varphi \circ \alpha_{1}=\alpha_{2}$ and $\varphi \circ \beta_{1}=\beta_{2}$.

Any faithful morphism $\varphi \in \operatorname{Mor}\left(C_{1}, C_{2}\right)$ with $\varphi \circ \alpha_{1}=\alpha_{2}$ and $\varphi \circ \beta_{1}=\beta_{2}$ satisfies $\varphi\left(C_{1}\right)=C_{2}$ and hence is a ${ }^{*}$-isomorphism.

Example 4.4. Let $C=\alpha(A) \cdot \beta(B)$ be a crossed product and $U \in \mathcal{U}(C)$. Then

$$
(C, \alpha, \beta) \simeq\left(C, \operatorname{Ad}_{U} \circ \alpha, \operatorname{Ad}_{U} \circ \beta\right) .
$$

We shall construct twisted tensor products using quantum group coactions on the tensor factors. First we generalise the notation of Heisenberg pairs for a given bicharacter in Section 4.1. There are two possible ways to commute generalised Heisenberg pairs (see Section 4.1.1). Ordinary commutation plays an important role in the construction of twisted tensor products. We propose two equivalent constructions of crossed products. At the level of $\mathrm{C}^{*}$-algebras we use coactions of two quantum groups, related through bicharacters, on $\mathrm{C}^{*}$-algebras and put them together using generalised Heisenberg pairs (see Section 4.2). The second approach deals with covariant representations of quantum group coactions on Hilbert spaces discussed in 
Section 4.3. In Section 4.4, we explore various properties of crossed products. Section 4.5 shows that our crossed product generalises the skew-commutative tensor product of $\mathbb{Z} / 2$-graded $\mathrm{C}^{*}$-algebras and the crossed product of coactions by a quantum group. Finally, in Section 4.6. we construct the generalised Drinfeld double, a $\mathrm{C}^{*}$-quantum group, using Drinfeld commutation of generalised Heisenberg pairs associated to a bicharacter.

\subsection{Heisenberg pairs revisited}

This section generalises the notions of Heisenberg and anti-Heisenberg pairs from reduced bicharacters, discussed in Section 2.4 to general bicharacters. We use them to construct our noncommutative tensor product, after establishing properties of Heisenberg pairs necessary for that purpose.

Let $\mathbb{G}=\left(A, \Delta_{A}\right)$ and $\mathbb{H}=\left(B, \Delta_{B}\right)$ be $\mathrm{C}^{*}$-quantum groups. Let $\mathrm{W}^{A} \in \mathcal{U}(\hat{A} \otimes A)$ and $\mathrm{W}^{B} \in \mathcal{U}(\hat{B} \otimes B)$ be their reduced bicharacters. Let $\chi \in \mathcal{U}(\hat{A} \otimes \hat{B})$ be a bicharacter from $A$ to $\hat{B}$. Heisenberg pairs and anti-Heisenberg pairs are pairs of representations $(\alpha, \beta)$ of $\hat{A}$ and $\hat{B}$ on the same Hilbert space $\mathcal{H}$ that satisfy suitable compatibility conditions.

We use these pairs to define twisted tensor products $C \otimes_{\chi} D$ in Section 4.2. A crucial technical point is to show that a pair of representations of $C$ and $D$ generates a crossed product $\mathrm{C}^{*}$-algebra. Here the commutativity result in Section 4.1.1 is crucial. In addition, we construct examples of $\chi$-Heisenberg pairs and $\chi$-anti-Heisenberg pairs, thus proving their existence, and give some equivalent characterisations of them.

Definition 4.5. A pair of representations $\alpha: A \rightarrow \mathbb{B}(\mathcal{H}), \beta: B \rightarrow \mathbb{B}(\mathcal{H})$ is called a $\chi$-Heisenberg pair or briefly Heisenberg pair if

$$
\mathrm{W}_{1 \alpha}^{A} \mathrm{~W}_{2 \beta}^{B}=\mathrm{W}_{2 \beta}^{B} \mathrm{~W}_{1 \alpha}^{A} \chi_{12} \quad \text { in } \mathcal{U}(\hat{A} \otimes \hat{B} \otimes \mathbb{K}(\mathcal{H}))
$$

here $\mathrm{W}_{1 \alpha}^{A}:=\left(\left(\operatorname{id}_{\hat{A}} \otimes \alpha\right) \mathrm{W}^{A}\right)_{13}$ and $\mathrm{W}_{2 \beta}^{B}:=\left(\left(\operatorname{id}_{\hat{B}} \otimes \beta\right) \mathrm{W}^{B}\right)_{23}$. It is called a $\chi$-anti-Heisenberg pair or briefly anti-Heisenberg pair if

$$
\mathrm{W}_{2 \beta}^{B} \mathrm{~W}_{1 \alpha}^{A}=\chi_{12} \mathrm{~W}_{1 \alpha}^{A} \mathrm{~W}_{2 \beta}^{B} \quad \text { in } \mathcal{U}(\hat{A} \otimes \hat{B} \otimes \mathbb{K}(\mathcal{H})),
$$

with similar conventions as above.

The case where $\mathbb{H}=\widehat{\mathbb{G}}$ and $\chi=\mathrm{W}^{A} \in \mathcal{U}(\hat{A} \otimes A)$ is the reduced bicharacter of $\mathbb{G}$ is already considered in Section 2.4

Lemma 4.6. Assume a pair of representations $(\pi, \hat{\pi})$ of $A$ and $\hat{A}$ on a Hilbert space $\mathcal{H}$. Then $(\pi, \hat{\pi})$ is a

1. $\mathrm{W}^{A}$-Heisenberg pair if and only if it is a $\mathbb{G}$-Heisenberg pair.

2. $\mathrm{W}^{A}$-anti-Heisenberg pair if and only if it is a $\mathbb{G}$-anti-Heisenberg pair.

Proof. Let $\pi$ and $\hat{\pi}$ be representations of $A$ and $\hat{A}$ on $\mathcal{H}$ satisfying $\left(2.12\right.$. When we apply $\sigma_{23}$ to both sides of 2.12 we get

$$
\left(\widehat{\mathrm{W}}_{2 \hat{\pi}}^{A}\right)^{*} \mathrm{~W}_{1 \pi}^{A}=\mathrm{W}_{1 \pi}^{A} \mathrm{~W}_{12}^{A}\left(\widehat{\mathrm{W}}_{2 \hat{\pi}}^{A}\right)^{*} \quad \text { in } \mathcal{U}(\hat{A} \otimes A \otimes \mathbb{K}(\mathcal{H})) .
$$

This is equivalent to $\mathrm{W}_{1 \pi}^{A} \widehat{\mathrm{W}}_{2 \hat{\pi}}^{A}=\widehat{\mathrm{W}}_{2 \hat{\pi}}^{A} \mathrm{~W}_{1 \pi}^{A} \mathrm{~W}_{12}^{A}$, which is 4.1 for $\hat{B}=A, \chi=\mathrm{W}^{A}, \alpha=\pi$ and $\beta=\hat{\pi}$. This computation may be reversed as well.

The computation for anti-Heisenberg pairs is similar.

Example 4.7. Let $\overline{\mathcal{H}}$ be the conjugate Hilbert space to the Hilbert space $\mathcal{H}$. Recall that the transpose of an operator $x \in \mathbb{B}(\mathcal{H})$ is the operator $x^{\top} \in \mathbb{B}(\overline{\mathcal{H}})$ defined by $x^{\top}(\bar{\xi}):=\overline{x^{*} \xi}$ for all $\xi \in \mathcal{H}$. The transposition is a linear, involutive anti-automorphism $\mathbb{B}(\mathcal{H}) \rightarrow \mathbb{B}(\overline{\mathcal{H}})$. Recall that the unitary antipode $\mathrm{R}_{A}: A \rightarrow A$ is also a linear, involutive anti-automorphism. Therefore, if $\alpha: A \rightarrow \mathbb{B}(\mathcal{H})$ and $\beta: B \rightarrow \mathbb{B}(\mathcal{H})$ are representations, then so are

$$
\begin{array}{rlrl}
\bar{\alpha}: A \rightarrow \mathbb{B}(\overline{\mathcal{H}}), & & a \mapsto\left(\mathrm{R}_{A}(a)\right)^{\top}, \\
\bar{\beta}: B \rightarrow \mathbb{B}(\overline{\mathcal{H}}), & b \mapsto\left(\mathrm{R}_{B}(b)\right)^{\top} .
\end{array}
$$


Lemma 4.8. The pair $(\alpha, \beta)$ is Heisenberg if and only if $(\bar{\alpha}, \bar{\beta})$ is anti-Heisenberg.

Proof. Let $(\alpha, \beta)$ be a Heisenberg pair. The following computation shows that $(\bar{\alpha}, \bar{\beta})$ is an anti-Heisenberg pair:

$$
\begin{aligned}
\mathrm{W}_{2 \bar{\beta}}^{B} \mathrm{~W}_{1 \bar{\alpha}}^{A} & =\left(\mathrm{R}_{\hat{A}} \otimes \mathrm{R}_{\hat{B}} \otimes \mathrm{T}\right)\left(\mathrm{W}_{1 \alpha}^{A} \mathrm{~W}_{2 \beta}^{B}\right) \\
& =\left(\mathrm{R}_{\hat{A}} \otimes \mathrm{R}_{\hat{B}} \otimes \mathrm{T}\right)\left(\mathrm{W}_{2 \beta}^{B} \mathrm{~W}_{1 \alpha}^{A} \chi_{12}\right)=\chi_{12} \mathrm{~W}_{1 \bar{\alpha}}^{A} \mathrm{~W}_{2 \bar{\beta}}^{B} ;
\end{aligned}
$$

the first equality uses $\left(\mathrm{R}_{\hat{A}} \otimes \mathrm{R}_{\hat{B}}\right) \chi=\chi$ (see Proposition 3.10 and the antimultiplicativity of $\mathrm{R}_{\hat{A}} \otimes \mathrm{R}_{\hat{B}} \otimes \mathrm{T}$; the second equality uses 4.1); and the third equality uses the same facts as for the previous ones in reverse order.

The computation above may be reversed to show the converse direction.

Thus Heisenberg pairs and anti-Heisenberg pairs are essentially equivalent.

Recall that a bicharacter $\chi$ gives rise to a dual bicharacter $\hat{\chi} \in \mathcal{U}(\hat{B} \otimes \hat{A})$ by $\sqrt{3.9}$ and to a right quantum group homomorphism $\Delta_{R}: A \rightarrow A \otimes \hat{B}$ with 3.15 . Similarly, $\hat{\chi}$ gives rise to a right quantum group homomorphism $\hat{\Delta}_{R}: B \rightarrow B \otimes \hat{A}$. We may reformulate the condition of being a Heisenberg pair in terms of $\hat{\chi}, \Delta_{R}$ and $\hat{\Delta}_{R}$, respectively:

Lemma 4.9. Let $\alpha$ and $\beta$ be representations of $A$ and $B$ on a Hilbert space $\mathcal{H}$. Then the following are equivalent:

1. $(\alpha, \beta)$ is a $\chi$-Heisenberg pair acting on $\mathcal{H}$;

2. $(\beta, \alpha)$ is a $\hat{\chi}$-Heisenberg pair acting on $\mathcal{H}$;

3. $\left(\alpha \otimes \operatorname{id}_{\hat{B}}\right) \Delta_{R}(a)=\left(\widehat{\mathrm{W}}_{\beta 2}^{B}\right)\left(\alpha(a) \otimes 1_{\hat{B}}\right)\left(\widehat{\mathrm{W}}^{B}\right)_{\beta 2}^{*}$ for all $a \in A$;

4. $\left(\beta \otimes \operatorname{id}_{\hat{A}}\right) \hat{\Delta}_{R}(b)=\left(\widehat{\mathrm{W}}_{\alpha 2}^{A}\right)\left(\beta(b) \otimes 1_{\hat{A}}\right)\left(\widehat{\mathrm{W}}^{A}\right)_{\alpha 2}^{*}$ for all $b \in B$.

Proof. (1) $\Longleftrightarrow(2)$ : (1) is equivalent to

$$
\mathrm{W}_{1 \alpha}^{A} \mathrm{~W}_{2 \beta}^{B} \chi_{12}^{*}=\mathrm{W}_{2 \beta}^{B} \mathrm{~W}_{1 \alpha}^{A} \quad \text { in } \mathcal{U}(\hat{A} \otimes \hat{B} \otimes \mathbb{K}(\mathcal{H}))
$$

by (4.1. Applying $\sigma_{12}$ gives

$$
\mathrm{W}_{2 \alpha}^{A} \mathrm{~W}_{1 \beta}^{B} \hat{\chi}_{12}=\mathrm{W}_{1 \beta}^{B} \mathrm{~W}_{2 \alpha}^{A} \quad \text { in } \mathcal{U}(\hat{B} \otimes \hat{A} \otimes \mathbb{K}(\mathcal{H})),
$$

which is equivalent to $(\beta, \alpha)$ being a $\hat{\chi}$-Heisenberg pair. Thus $(1) \Longleftrightarrow(2)$.

$(1) \Longleftrightarrow(3)$ : Let $(\alpha, \beta)$ be a Heisenberg pair. The following computation takes place in $\mathcal{U}(\hat{A} \otimes \mathbb{K}(\mathcal{H}) \otimes \hat{B})$ :

$$
\begin{aligned}
\left(\mathrm{id}_{\hat{A}} \otimes \alpha \otimes \mathrm{id}_{\hat{B}}\right)\left(\mathrm{id}_{\hat{A}} \otimes \Delta_{R}\right) \mathrm{W}^{A} & =\mathrm{W}_{1 \alpha}^{A} \chi_{13}=\sigma_{23}\left(\mathrm{~W}_{1 \alpha}^{A} \chi_{12}\right) \\
& =\sigma_{23}\left(\left(\mathrm{~W}_{2 \beta}^{B}\right)^{*} \mathrm{~W}_{1 \alpha}^{A} \mathrm{~W}_{2 \beta}^{B}\right)=\left(\widehat{\mathrm{W}}_{\beta 3}^{B}\right) \mathrm{W}_{1 \alpha}^{A}\left(\widehat{\mathrm{W}}_{\beta 3}^{B}\right)^{*} ;
\end{aligned}
$$

the first equality uses 3.15 ; the second equality is obvious; the third equality uses 4.1); and the last equality uses $\widehat{\mathrm{W}}^{B}=\sigma\left(\left(\mathrm{W}^{B}\right)^{*}\right)$. Since $\left\{\left(\omega \otimes \mathrm{id}_{A}\right) \mathrm{W}^{A}: \omega \in \hat{A}^{\prime}\right\}$ is linearly dense in $A$, slicing the first leg of the first and the last expression of the above equation yields $(1) \Longrightarrow(3)$.

Conversely, applying $\operatorname{id}_{\hat{A}} \otimes \alpha \otimes \operatorname{id}_{\hat{B}}$ on both sides of 3.15 and using (3), we get

$$
\mathrm{W}_{1 \alpha}^{A} \chi_{13}=\left(\operatorname{id}_{\hat{A}} \otimes\left(\alpha \otimes \operatorname{id}_{\hat{B}}\right) \Delta_{R}\right) \mathrm{W}^{A}=\left(\widehat{\mathrm{W}}_{\beta 3}^{B}\right) \mathrm{W}_{1 \alpha}^{A}\left(\widehat{\mathrm{W}}_{\beta 3}^{B}\right)^{*} \quad \text { in } \mathcal{U}(\hat{A} \otimes \mathbb{K}(\mathcal{H}) \otimes \hat{B}) ;
$$

applying $\sigma_{23}$ to this gives 4.1 . Thus $(3) \Longrightarrow(1)$.

To prove $(2) \Longleftrightarrow(4)$ argue as in the proof that $(1) \Longleftrightarrow(3)$.

Unlike $\mathbb{G}$-Heisenberg pairs (Proposition 2.21), $\chi$-Heisenberg pairs are not faithful in general.

Example 4.10. Assume that $\mathbb{G}$ and $\mathbb{H}$ have bounded counits $\mathrm{e}_{A}: A \rightarrow \mathbb{C}$ and $\mathrm{e}_{B}: B \rightarrow \mathbb{C}$. Proposition $31(2)$ in 39 gives $\left(\mathrm{id}_{\hat{A}} \otimes \mathrm{e}_{A}\right) \mathrm{W}^{A}=1_{\hat{A}}$ and $\left(\mathrm{id}_{\hat{B}} \otimes \mathrm{e}_{B}\right) \mathrm{W}^{B}=1_{\hat{B}}$; hence $\left(\mathrm{e}_{A}, \mathrm{e}_{B}\right)$ is a $\chi$-Heisenberg pair for the trivial bicharacter $\chi=1_{\hat{A}} \otimes 1_{\hat{B}}$ and clearly not faithful. 
Lemma 4.11. Let $(\pi, \hat{\pi})$ and $(\eta, \hat{\eta})$ be $\mathbb{G}$ - and $\mathbb{H}$-Heisenberg pairs on Hilbert spaces $\mathcal{H}_{\pi}$ and $\mathcal{H}_{\eta}$, respectively. Then the pair of representations $(\alpha, \beta)$ of $A$ and $B$ on $\mathcal{H}_{\pi} \otimes \mathcal{H}_{\eta}$ defined by $\alpha(a):=(\pi \otimes \hat{\eta}) \Delta_{R}(a)$ and $\beta(b):=1_{\mathcal{H}_{\pi}} \otimes \eta(b)$ is a $\chi$-Heisenberg pair; here $\Delta_{R}: A \rightarrow A \otimes \hat{B}$ is the right quantum group homomorphism associated to $\chi$ as in Theorem 3.21. Similarly, the pair of representations $\left(\alpha^{\prime}, \beta^{\prime}\right)$ of $A$ and $B$ on $\mathcal{H}_{\eta} \otimes \mathcal{H}_{\pi}$ defined by $\alpha(a):=1_{\mathcal{H}_{\eta}} \otimes \pi(a)$ and $\beta(b):=\left(\eta \otimes \hat{\pi}_{)} \hat{\Delta}_{R}(b)\right.$ is a $\chi$-Heisenberg pair; here $\hat{\Delta}_{R}: B \rightarrow B \otimes \hat{A}$ is the right quantum group homomorphism associated to $\hat{\chi}$.

Proof. First we check the following equation:

$$
\chi_{1 \hat{\eta}} \mathrm{W}_{2 \eta}^{B}=\mathrm{W}_{2 \eta}^{B} \chi_{1 \hat{\eta}} \chi_{12} \quad \text { in } \mathcal{U}\left(\hat{A} \otimes \hat{B} \otimes \mathbb{K}\left(\mathcal{H}_{\eta}\right)\right) .
$$

The coaction $\hat{B} \rightarrow \hat{B} \otimes \hat{B}$ associated to the reduced bicharacter $\mathrm{W}^{B}$ is the usual comultiplication $\hat{\Delta}_{B}$. Hence

$$
\left(\widehat{\mathrm{W}}_{\eta 3}^{B}\right) \chi_{1 \hat{\eta}}\left(\widehat{\mathrm{W}}_{\eta 3}^{B}\right)^{*}=\left(\mathrm{id}_{\hat{A}} \otimes \hat{\eta} \otimes \mathrm{id}_{\hat{B}}\right)\left(\mathrm{id} \otimes \hat{\Delta}_{B}\right) \chi=\left(\operatorname{id}_{\hat{A}} \otimes \hat{\eta} \otimes \operatorname{id}_{\hat{B}}\right)\left(\chi_{12} \chi_{13}\right)=\chi_{1 \hat{\eta}} \chi_{13}
$$

in $\mathcal{U}\left(\hat{A} \otimes \mathbb{K}\left(\mathcal{H}_{\eta}\right) \otimes \hat{B}\right)$ because of Lemma $4.9(4)$ and the bicharacter property 3.2$)$ of $\chi$. When we flip the last two legs, we turn $\widehat{\mathrm{W}}_{\eta 3}^{B}$ into $\left(\mathrm{W}_{2 \eta}^{B}\right)^{*}$. Rearranging then gives 4.4.

Now we can check that $(\alpha, \beta)$ is a Heisenberg pair. The following computation takes place in $\mathcal{U}(\hat{A} \otimes$ $\left.\hat{B} \otimes \mathbb{K}\left(\mathcal{H}_{\pi}\right) \otimes \mathbb{K}\left(\mathcal{H}_{\eta}\right)\right):$

$$
\mathrm{W}_{1 \alpha}^{A} \mathrm{~W}_{2 \beta}^{B}=\mathrm{W}_{1 \pi}^{A} \chi_{1 \hat{\eta}} \mathrm{W}_{2 \eta}^{B}=\mathrm{W}_{1 \pi}^{A} \mathrm{~W}_{2 \eta}^{B} \chi_{1 \hat{\eta}} \chi_{12}=\mathrm{W}_{2 \eta}^{B} \mathrm{~W}_{1 \pi}^{A} \chi_{1 \hat{\eta}} \chi_{12}=\mathrm{W}_{2 \beta}^{B} \mathrm{~W}_{1 \alpha}^{A} \chi_{12}
$$

the first equality uses the definitions of $\alpha$ and $\beta$ and (3.15); the second equality uses 4.4p; the third equality uses that $\mathrm{W}_{1 \pi}^{A}$ and $\mathrm{W}_{2 \eta}^{B}$ commute; and the fourth equality uses the definitions of $\alpha$ and $\beta$ again.

A similar argument shows that $\left(\alpha^{\prime}, \beta^{\prime}\right)$ is a $\chi$-Heisenberg pair.

\subsubsection{Commutativity and Heisenberg pairs}

It is interesting to observe how the commutation relations look like when we put a Heisenberg and an anti-Heisenberg pair together. There are only two possible ways to do so. Ordinary commutativity comes out if we put together a Heisenberg and an anti-Heisenberg pair. This will be crucial for the existence of our noncommutative tensor product.

Proposition 4.12. Let $\mathcal{H}$ and $\mathcal{K}$ be Hilbert spaces; let $\alpha$ and $\beta$ be representations of $A$ and $B$ on $\mathcal{H}$, respectively; and let $\bar{\alpha}$ and $\bar{\beta}$ be representations of $A$ and $B$ on $\mathcal{K}$, respectively. Then the following are equivalent:

1. the representations $(\alpha \otimes \bar{\alpha}) \Delta_{A}$ and $(\beta \otimes \bar{\beta}) \Delta_{B}$ of $A$ and $B$ on $\mathcal{H} \otimes \mathcal{K}$ commute, that is, for any a $\in A$ and $b \in B$, we have

$$
\left[(\alpha \otimes \bar{\alpha}) \Delta_{A}(a),(\beta \otimes \bar{\beta}) \Delta_{B}(b)\right]=0 ;
$$

2. there is a bicharacter $\chi \in \mathcal{U}(\hat{A} \otimes \hat{B})$ such that $(\alpha, \beta)$ is a Heisenberg pair and $(\bar{\alpha}, \bar{\beta})$ is an antiHeisenberg pair.

Proof. Equation 4.5 is equivalent to

$$
\mathrm{W}_{1 \alpha}^{A} \mathrm{~W}_{1 \bar{\alpha}}^{A} \mathrm{~W}_{2 \beta}^{B} \mathrm{~W}_{2 \bar{\beta}}^{B}=\mathrm{W}_{2 \beta}^{B} \mathrm{~W}_{2 \bar{\beta}}^{B} \mathrm{~W}_{1 \alpha}^{A} \mathrm{~W}_{1 \bar{\alpha}}^{A} \quad \text { in } \mathcal{U}(\hat{A} \otimes \hat{B} \otimes \mathbb{K}(\mathcal{H}) \otimes \mathbb{K}(\mathcal{K}))
$$

because of 2.8 and $\sqrt{2.5}$ for $\mathrm{W}^{A}$ and $\mathrm{W}^{B}$. We may commute $\mathrm{W}_{1 \bar{\alpha}}^{A}$ with $\mathrm{W}_{2 \beta}^{B}$ and $\mathrm{W}_{2 \bar{\beta}}^{B}$ with $\mathrm{W}_{1 \alpha}^{A}$ on both sides and rearrange 4.6 to

$$
\chi:=\left(\mathrm{W}^{A}\right)_{1 \alpha}^{*}\left(\mathrm{~W}^{B}\right)_{2 \beta}^{*} \mathrm{~W}_{1 \alpha}^{A} \mathrm{~W}_{2 \beta}^{B}=\mathrm{W}_{2 \bar{\beta}}^{B} \mathrm{~W}_{1 \bar{\alpha}}^{A}\left(\mathrm{~W}^{B}\right)_{2 \bar{\beta}}^{*}\left(\mathrm{~W}^{A}\right)_{1 \bar{\alpha}}^{*} .
$$

This implies $\chi \in \mathcal{U}(\hat{A} \otimes \hat{B})$ because its first definition has $1_{\mathcal{K}}$ in the fourth leg and its second definition has $1_{\mathcal{H}}$ in the third leg. 
We check that $\chi$ is a bicharacter in both legs. First we check (3.1):

$$
\begin{aligned}
\left(\hat{\Delta}_{A} \otimes \operatorname{id}_{\hat{B}}\right) \chi & =\left(\hat{\Delta}_{A} \otimes \operatorname{id}_{\hat{B}}\right)\left(\mathrm{W}_{2 \bar{\beta}}^{B} \mathrm{~W}_{1 \bar{\alpha}}^{A}\left(\mathrm{~W}^{B}\right)_{2 \bar{\beta}}^{*}\left(\mathrm{~W}^{A}\right)_{1 \bar{\alpha}}^{*}\right) \\
& =\mathrm{W}_{3 \bar{\beta}}^{B} \mathrm{~W}_{2 \bar{\alpha}}^{A} \mathrm{~W}_{1 \bar{\alpha}}^{A}\left(\mathrm{~W}^{B}\right)_{3 \bar{\beta}}^{*}\left(\mathrm{~W}^{A}\right)_{1 \bar{\alpha}}^{*}\left(\mathrm{~W}^{A}\right)_{2 \bar{\alpha}}^{*} \\
& =\chi_{23} \mathrm{~W}_{2 \bar{\alpha}}^{A} \chi_{13}\left(\mathrm{~W}^{A}\right)_{2 \bar{\alpha}}^{*}=\chi_{23} \chi_{13} ;
\end{aligned}
$$

the first and third equality use the second definition of $\chi$ in 4.7$)$; the second equality uses $(3.1)$ for $\mathrm{W}^{A}$; and the last equality uses that $\mathrm{W}_{2 \bar{\alpha}}^{A}$ and $\chi_{13}$ commute. A similar computation using the first definition of $\chi$ in 4.7 yields $\left(\operatorname{id}_{\hat{A}} \otimes \hat{\Delta}_{B}\right) \chi=\chi_{12} \chi_{13}$; thus $\chi \in \mathcal{U}(\hat{A} \otimes \hat{B})$ is a bicharacter.

The first definition of $\chi$ in 4.7 says that $(\alpha, \beta)$ is a Heisenberg pair, and the second one says that $(\bar{\alpha}, \bar{\beta})$ is an anti-Heisenberg pair.

Conversely, if $\chi \in \mathcal{U}(\hat{A} \otimes \hat{B})$ is a bicharacter, $(\alpha, \beta)$ a Heisenberg pair and $(\bar{\alpha}, \bar{\beta})$ an anti-Heisenberg pair, then 4.7) follows, and hence 4.5.

If we put an anti-Heisenberg pair and a Heisenberg pair together we get twisted commutation. This will eventually become the central ingredient to construct the generalised Drinfeld double discussed in Section 4.6 and extend the notion of quasitriangularity to the $\mathrm{C}^{*}$-algebraic framework in Chapter 5.

Definition 4.13. A pair $(\rho, \theta)$ of representations of $A$ and $B$ on a Hilbert space $\mathcal{H}$ is a $\chi$-Drinfeld pair if

$$
\chi_{12} \mathrm{~W}_{1 \rho}^{A} \mathrm{~W}_{2 \theta}^{B}=\mathrm{W}_{2 \theta}^{B} \mathrm{~W}_{1 \rho}^{A} \chi_{12} \quad \text { in } \mathcal{U}(\hat{A} \otimes \hat{B} \otimes \mathbb{K}(\mathcal{H})) .
$$

In particular, when $\chi=\mathrm{W}^{A}$, we call $\mathrm{W}^{A}$-Drinfeld pairs $\mathbb{G}$-Drinfeld pairs. Equivalently, a pair of representations $\rho: A \rightarrow \mathbb{B}(\mathcal{H})$ and $\theta: \hat{A} \rightarrow \mathbb{B}(\mathcal{H})$ satisfies the $\mathbb{G}$-Drinfeld-commutation relation if and only if

$$
\mathrm{W}_{1 \rho}^{A} \mathrm{~W}_{13}^{A} \mathrm{~W}_{\theta 3}^{A}=\mathrm{W}_{\theta 3}^{A} \mathrm{~W}_{13}^{A} \mathrm{~W}_{1 \rho}^{A} \quad \text { in } \mathcal{U}(\hat{A} \otimes \mathbb{K}(\mathcal{H}) \otimes A) .
$$

Proposition 4.14. Let $(\alpha, \beta)$ and $(\bar{\alpha}, \bar{\beta})$ be a $\chi$-Heisenberg and $\chi$-anti-Heisenberg pair on $\mathcal{H}$ and $\mathcal{K}$, respectively. Define the representations $\rho:=(\bar{\alpha} \otimes \alpha) \Delta_{A}$ and $\theta:=(\bar{\beta} \otimes \beta) \Delta_{B}$ of $A$ and $B$ on $\mathcal{K} \otimes \mathcal{H}$. Then $(\rho, \theta)$ is a $\chi$-Drinfeld pair on $\mathcal{K} \otimes \mathcal{H}$.

Proof. We must check 4.8 for $(\rho, \theta)$. Equations 2.8, 4.1] and 4.2 yield:

$$
\begin{aligned}
\chi_{12} \mathrm{~W}_{1 \rho}^{A} \mathrm{~W}_{2 \theta}^{B}=\chi_{12} \mathrm{~W}_{1 \bar{\alpha}}^{A} \mathrm{~W}_{1 \alpha}^{A} \mathrm{~W}_{2 \bar{\beta}}^{B} \mathrm{~W}_{2 \beta}^{B}=\chi_{12} \mathrm{~W}_{1 \bar{\alpha}}^{A} \mathrm{~W}_{2 \bar{\beta}}^{B} \mathrm{~W}_{1 \alpha}^{A} \mathrm{~W}_{2 \beta}^{B} & =\mathrm{W}_{2 \bar{\beta}}^{B} \mathrm{~W}_{1 \bar{\alpha}}^{A} \mathrm{~W}_{2 \beta}^{B} \mathrm{~W}_{1 \alpha}^{A} \chi_{12} \\
& =\mathrm{W}_{2 \bar{\beta}}^{B} \mathrm{~W}_{2 \beta}^{B} \mathrm{~W}_{1 \bar{\alpha}}^{A} \mathrm{~W}_{1 \alpha}^{A} \chi_{12}=\mathrm{W}_{2 \theta}^{B} \mathrm{~W}_{1 \rho}^{A} \chi_{12}
\end{aligned}
$$

because $\mathrm{W}_{1 \alpha}^{A}$ commutes with $\mathrm{W}_{2 \bar{\beta}}^{B}$ and $\mathrm{W}_{1 \bar{\alpha}}^{A}$ commutes with $\mathrm{W}_{2 \beta}^{B}$.

Corollary 4.15. Let $(\pi, \hat{\pi})$ and $(\eta, \hat{\eta})$ be $\mathbb{G}$-Heisenberg and $\mathbb{G}$-anti-Heisenberg pairs acting on $\mathcal{H}_{\pi}$ and $\mathcal{H}_{\eta}$, respectively. Then the representations $\rho: A \rightarrow \mathbb{B}\left(\mathcal{H}_{\pi} \otimes \mathcal{H}_{\eta}\right)$ and $\theta: \hat{A} \rightarrow \mathbb{B}\left(\mathcal{H}_{\pi} \otimes \mathcal{H}_{\eta}\right)$ defined by $\rho(a):=$ $(\eta \otimes \pi) \Delta_{A}(a)$ and $\theta(\hat{a}):=(\hat{\eta} \otimes \hat{\pi}) \hat{\Delta}_{A}(\hat{a})$ satisfy the $\mathbb{G}$-Drinfeld commutation relation.

\subsection{Twisted tensor products via Heisenberg pairs}

Let $\mathbb{G}=\left(A, \Delta_{A}\right)$ and $\mathbb{H}=\left(B, \Delta_{B}\right)$ be $C^{*}$-quantum groups, let $\chi \in \mathcal{U}(\hat{A} \otimes \hat{B})$ be a bicharacter, let $(C, \gamma)$ be a $\mathbb{G}-C^{*}$-algebra, and let $(D, \delta)$ be an $\mathbb{H}-\mathrm{C}^{*}$-algebra. Let $(\alpha, \beta)$ be a $\chi$-Heisenberg pair on some Hilbert space $\mathcal{H}$.

Using this data, we now construct a crossed product $\left(C \nabla_{\chi} D, \iota_{C}, \iota_{D}\right)$ of $C$ and $D$ in the sense of Definition 4.1. A more precise notation is

$$
C \otimes_{\chi} D=(C, \gamma) \otimes_{\chi}(D, \delta)
$$

There is no need to mention $(\alpha, \beta)$ in our notation because all Heisenberg pairs give equivalent crossed products; we will prove this in Section 4.3 . 
Lemma 4.16. Define morphisms

$$
\begin{aligned}
\iota_{C}: C \rightarrow C \otimes D \otimes \mathbb{K}(\mathcal{H}), & c \mapsto\left(\operatorname{id}_{C} \otimes \alpha\right) \gamma(c)_{13}, \\
\iota_{D}: D \rightarrow C \otimes D \otimes \mathbb{K}(\mathcal{H}), & d \mapsto\left(\operatorname{id}_{D} \otimes \beta\right) \delta(d)_{23} .
\end{aligned}
$$

Then $\iota_{C}(C) \cdot \iota_{D}(D)=\iota_{D}(D) \cdot \iota_{C}(C)$ in $\mathcal{M}(C \otimes D \otimes \mathbb{K}(\mathcal{H}))$.

It follows that

$$
C \otimes_{\chi} D:=\iota_{C}(C) \cdot \iota_{D}(D)
$$

is a $\mathrm{C}^{*}$-algebra and that $\iota_{C}$ and $\iota_{D}$ are morphisms from $C$ and $D$ to $C \otimes_{\chi} D$, respectively. Thus $\left(C \otimes_{\chi} D, \iota_{C}, \iota_{D}\right)$ is a crossed product of $C$ and $D$ in the sense of Definition 4.1 .

Lemma 4.17. Let $X \subseteq C$ and $Y \subseteq D$ be closed subspaces with

$$
\gamma(X) \cdot\left(1_{C} \otimes A\right)=X \otimes A \quad \text { and } \quad \delta(Y) \cdot\left(1_{D} \otimes B\right)=Y \otimes B .
$$

Then $\iota_{C}(X) \cdot \iota_{Y}(D)=\iota_{D}(Y) \cdot \iota_{C}(X)$ in $\mathcal{M}(C \otimes D \otimes \mathbb{K}(\mathcal{H}))$.

Proof. Since our coactions satisfy the Podleś conditions, Lemma 4.16 is the special case $X=C$ and $Y=D$ of Lemma 4.17. Hence it is enough to verify Lemma 4.17

Let $(\bar{\alpha}, \beta)$ be a $\chi$-anti Heisenberg pair on a Hilbert space $\mathcal{K}$. The definition of $\iota_{C}$ and the comodule property 2.22 for $\gamma$ yield

$$
\left(\iota_{C} \otimes \bar{\alpha}\right) \gamma=\left(\left(\operatorname{id}_{C} \otimes \alpha \otimes \bar{\alpha}\right)\left(\gamma \otimes \operatorname{id}_{A}\right) \gamma\right)_{134}=\left(\left(\operatorname{id}_{C} \otimes(\alpha \otimes \bar{\alpha}) \Delta_{A}\right) \gamma\right)_{134}
$$

Similarly,

$$
\left(\iota_{D} \otimes \bar{\beta}\right) \delta=\left(\left(\operatorname{id}_{D} \otimes(\beta \otimes \bar{\beta}) \Delta_{B}\right) \delta\right)_{234} .
$$

Now Proposition 4.12 yields

$$
\left(\iota_{C} \otimes \bar{\alpha}\right) \gamma(c) \cdot\left(\iota_{D} \otimes \bar{\beta}\right) \delta(d)=\left(\iota_{D} \otimes \bar{\beta}\right) \delta(d) \cdot\left(\iota_{C} \otimes \bar{\alpha}\right) \gamma(c)
$$

for all $c \in C, d \in D$.

Since $\bar{\alpha}(A) \cdot \mathbb{K}(\mathcal{K})=\mathbb{K}(\mathcal{K})$, our assumption $\gamma(X) \cdot\left(1_{C} \otimes A\right)=X \otimes A$ gives

$$
\begin{aligned}
\left(\left(\iota_{C} \otimes \bar{\alpha}\right) \gamma(X)\right) \cdot \mathbb{K}(\mathcal{K})_{4}=\left(\iota_{C} \otimes \bar{\alpha}\right)(\gamma(X) & \left.\cdot\left(1_{C} \otimes A\right)\right) \cdot \mathbb{K}(\mathcal{K})_{4} \\
& =\left(\iota_{C}(X) \otimes \bar{\alpha}(A)\right) \cdot\left(1_{C} \otimes 1_{\mathcal{H}} \otimes \mathbb{K}(\mathcal{K})\right)_{134}=\iota_{C}(X) \otimes \mathbb{K}(\mathcal{K}) .
\end{aligned}
$$

Similarly, $\bar{\beta}(B) \cdot \mathbb{K}(\mathcal{K})=\mathbb{K}(\mathcal{K})$, and the Podleś condition for $\delta$ gives

$$
\left(\left(\iota_{D} \otimes \bar{\beta}\right) \delta(Y)\right) \cdot \mathbb{K}(\mathcal{K})_{4}=\iota_{D}(Y) \otimes \mathbb{K}(\mathcal{K}) .
$$

Equation 4.10 gives

$$
\left(\iota_{C} \otimes \bar{\alpha}\right) \gamma(X) \cdot\left(\iota_{D} \otimes \bar{\beta}\right) \delta(Y)=\left(\iota_{D} \otimes \bar{\beta}\right) \delta(Y) \cdot\left(\iota_{C} \otimes \bar{\alpha}\right) \gamma(X) .
$$

Multiplying this equation on the right with $1_{C \otimes D \otimes \mathcal{H}} \otimes \mathbb{K}(\mathcal{K})$ and using the computations above to simplify, we get

$$
\left(\iota_{C}(X) \cdot \iota_{D}(Y)\right) \otimes \mathbb{K}(\mathcal{K})=\left(\iota_{D}(Y) \cdot \iota_{C}(X)\right) \otimes \mathbb{K}(\mathcal{K}) .
$$

Applying a state $\omega$ on $\mathbb{K}(\mathcal{K})$ to this equation gives $\iota_{C}(X) \cdot \iota_{D}(Y)=\iota_{D}(Y) \cdot \iota_{C}(X)$ as desired.

The following observation is useful to study slice maps on $C \otimes_{\chi} D$.

Lemma 4.18. In the situation of the previous lemma,

$$
\iota_{C}(X) \cdot \iota_{D}(Y) \cdot \mathbb{K}(\mathcal{H})_{3}=X \otimes Y \otimes \mathbb{K}(\mathcal{H}),
$$

where the right hand side means the closed linear span of $x \otimes y \otimes z$ with $x \in X, y \in Y, z \in \mathbb{K}(\mathcal{H})$. In particular, $\left(C \otimes_{\chi} D\right) \cdot \mathbb{K}(\mathcal{H})_{3}=C \otimes D \otimes \mathbb{K}(\mathcal{H})$. 
Proof. Since $\mathbb{K}(\mathcal{H})=\beta(B) \cdot \mathbb{K}(\mathcal{H})$, we may compute

$$
\iota_{D}(Y) \cdot \mathbb{K}(\mathcal{H})_{3}=\left(\left(\operatorname{id}_{D} \otimes \beta\right)\left(\delta(Y) \cdot\left(1_{D} \otimes B\right)\right)\right)_{23} \mathbb{K}(\mathcal{H})_{3}=(Y \otimes \beta(B) \cdot \mathbb{K}(\mathcal{H}))_{23}=\mathbb{K}(\mathcal{H})_{3} \cdot Y_{2}
$$

Here $Y_{2}$ and $\mathbb{K}(\mathcal{H})_{3}$ mean $Y$ and $\mathbb{K}(\mathcal{H})$ in the second and third leg, respectively. A similar computation for $\iota_{C}(X)$ using $\mathbb{K}(\mathcal{H})=\alpha(A) \cdot \mathbb{K}(\mathcal{H})$ now gives 4.11.

We will consider interesting examples of $\otimes_{\chi}$ in Section 4.5. Here we only mention a trivial example:

Example 4.19. Assume the coaction $\gamma$ is trivial. Then $\gamma(c)_{13}=c \otimes 1 \otimes 1$, so that $C \otimes_{\chi} D \cong C \otimes D$, embedded into $\mathcal{M}(C \otimes D \otimes \mathbb{K}(\mathcal{H}))$ via $\operatorname{id}_{C} \otimes\left(\operatorname{id}_{D} \otimes \beta\right) \delta$. We get the same conclusion if $\delta$ is trivial.

Lemma 4.20. Let $C_{0}$ be a $\mathrm{C}^{*}$-algebra with trivial $\mathbb{G}$-coaction and equip $C_{0} \otimes C$ with the coaction $\mathrm{id}_{C_{0}} \otimes \gamma$. Then

$$
\left(C_{0} \otimes C\right) \otimes_{\chi} D=C_{0} \otimes\left(C \otimes_{\chi} D\right) .
$$

A similar statement holds in the second variable.

Proof. The map $\iota_{C_{0} \otimes C}$ is $\operatorname{id}_{C_{0}} \otimes \iota_{C}$.

\subsection{Hilbert space representation of the twisted tensor prod- uct}

Definition 4.21. A covariant representation of $(C, \gamma, A)$ on a Hilbert space $\mathcal{H}$ is a pair consisting of a corepresentation $\mathrm{U} \in \mathcal{U}(\mathbb{K}(\mathcal{H}) \otimes A)$ and a representation $\varphi: C \rightarrow \mathbb{B}(\mathcal{H})$ that satisfy the covariance condition

$$
\left(\varphi \otimes \operatorname{id}_{A}\right) \circ \gamma(c)=\mathrm{U}\left(\varphi(c) \otimes 1_{A}\right) \mathrm{U}^{*} \quad \text { in } \mathcal{U}(\mathbb{K}(\mathcal{H}) \otimes A)
$$

for all $c \in C$. A faithful covariant representation is one where $\varphi$ is faithful.

Example 4.22. Let $\varphi_{0}: C \rightarrow \mathbb{B}\left(\mathcal{H}_{0}\right)$ be any faithful Hilbert space representation. Let $(\pi, \hat{\pi})$ be a faithful $\mathbb{G}$-Heisenberg pair on a Hilbert space $\mathcal{H}_{\pi}$; this exists because of Example 2.18. Let $\mathcal{H}:=\mathcal{H}_{0} \otimes \mathcal{H}_{\pi}$ and identify $\mathbb{K}(\mathcal{H}) \cong \mathbb{K}\left(\mathcal{H}_{0}\right) \otimes \mathbb{K}\left(\mathcal{H}_{\pi}\right)$. The unitary $\mathrm{U}:=1_{\mathcal{H}_{0}} \otimes \mathrm{W}_{\tilde{\pi} 2}^{A} \in \mathcal{U}(\mathbb{K}(\mathcal{H}) \otimes A)$ is a corepresentation; since $\varphi_{0}, \pi$ and $\gamma$ are faithful morphisms, $\varphi:=\left(\varphi_{0} \otimes \pi\right) \circ \gamma: C \rightarrow \mathbb{B}(\mathcal{H})$ is a faithful representation. The following computation in $\mathcal{M}\left(C \otimes \mathbb{K}\left(\mathcal{H}_{\pi}\right) \otimes A\right)$ implies the covariance condition for $(\varphi, \mathrm{U})$ :

$$
\left(\left(\operatorname{id}_{C} \otimes \pi\right) \gamma \otimes \operatorname{id}_{A}\right) \gamma(c)=\left(\operatorname{id}_{C} \otimes\left(\pi \otimes \operatorname{id}_{A}\right) \Delta_{A}\right) \gamma(c)=\left(\mathrm{W}_{\hat{\pi} 3}^{A}\right)\left(\gamma(c) \otimes 1_{A}\right)\left(\mathrm{W}_{\hat{\pi} 3}^{A}\right)^{*}
$$

for all $c \in C$, where we used 2.22 and Lemma 4.93 with $B=\hat{A}$ and $\Delta_{R}=\Delta_{A}$.

We are now going to construct a faithful Hilbert space representation of $C \otimes_{\chi} D$ using covariant Hilbert space representations of $(C, \gamma)$ and $(D, \delta)$. This yields an alternative definition of $C \nabla_{\chi} D$ and shows that $C \nabla_{\chi} D$ does not depend on the Heisenberg pair used in its construction.

Our new construction uses faithful covariant representations $\left(\varphi, \mathrm{U}^{\mathcal{H}}\right)$ of $(C, \gamma, A)$ and $\left(\psi, \mathrm{U}^{\mathcal{K}}\right)$ of $(D, \delta, B)$ on Hilbert spaces $\mathcal{H}$ and $\mathcal{K}$, respectively. (Example 4.22 shows that such faithful covariant representations always exists.)

The bicharacter $\chi$ and the corepresentations provide a unitary operator $Z$ on $\mathcal{H} \otimes \mathcal{K}$, generalising Proposition 2.38, as follows:

Theorem 4.23. Let $\mathrm{U}^{\mathcal{H}} \in \mathcal{U}(\mathcal{H} \otimes A)$ and $\mathrm{U}^{\mathcal{K}} \in \mathcal{U}(\mathcal{K} \otimes B)$ be corepresentations of $\mathbb{G}$ and $\mathbb{H}$, respectively. Then there is a unique unitary $Z \in \mathcal{U}(\mathcal{H} \otimes \mathcal{K})$ that satisfies

$$
\mathrm{U}_{1 \alpha}^{\mathcal{H}} \mathrm{U}_{2 \beta}^{\mathcal{K}} Z_{12}=\mathrm{U}_{2 \beta}^{\mathcal{K}} \mathrm{U}_{1 \alpha}^{\mathcal{H}} \quad \text { in } \mathcal{U}(\mathcal{H} \otimes \mathcal{K} \otimes \mathcal{L})
$$

for any $\chi$-Heisenberg pair $(\alpha, \beta)$ on some Hilbert space $\mathcal{L}$. 
With this unitary $Z$, define representations $\varphi_{1}$ and $\tilde{\psi}_{2}$ of $C$ and $D$ on $\mathcal{H} \otimes \mathcal{K}$ by

$$
\begin{aligned}
\varphi_{1}(c) & :=\varphi(c) \otimes 1_{\mathcal{K}}, \\
\tilde{\psi}_{2}(d) & :=Z\left(1_{\mathcal{H}} \otimes \psi(d)\right) Z^{*} .
\end{aligned}
$$

We can now formulate the main theorem of this section:

Theorem 4.24. Let $\left(\varphi, \mathrm{U}^{\mathcal{H}}\right)$ and $\left(\psi, \mathrm{U}^{\mathcal{K}}\right)$ be faithful covariant representations of $(C, \gamma, A)$ and $(D, \delta, B)$ on Hilbert spaces $\mathcal{H}$ and $\mathcal{K}$, respectively. Construct $\varphi_{1}$ and $\tilde{\psi}_{2}$ as above. Then there is a unique faithful representation $\rho: C \otimes_{\chi} D \rightarrow \mathbb{B}(\mathcal{H} \otimes \mathcal{K})$ with $\rho \circ \iota_{C}=\varphi_{1}$ and $\rho \circ \iota_{D}=\tilde{\psi}_{2}$.

Example 4.25. If $\chi=1$, then we may take $Z=1$. Thus $\tilde{\psi}_{2}=\psi_{2}$ and the crossed product is simply the minimal tensor product $C \otimes D$.

In the rest of this section, we prove the claims above and use the main theorem to show that the twisted tensor product does not depend on auxiliary choices.

Proof of Theorem 4.23. The uniqueness of $Z$ is clear from

$$
Z_{12}=\left(\mathrm{U}_{2 \beta}^{\mathcal{K}}\right)^{*}\left(\mathrm{U}_{1 \alpha}^{\mathcal{H}}\right)^{*} \mathrm{U}_{2 \beta}^{\mathcal{K}} \mathrm{U}_{1 \alpha}^{\mathcal{H}} .
$$

Existence means that the operator on the right acts identically on the third leg and does not depend on the Heisenberg pair. The quickest way to prove this uses universal quantum groups to turn corepresentations into representations.

By (2.34), there is a universal (left) corepresentation $\tilde{\mathcal{V}}^{A} \in \mathcal{U}\left(\hat{A}^{\mathrm{u}} \otimes A\right)$; its universal property gives a unique representation $\rho_{1}: \hat{A}^{\mathrm{u}} \rightarrow \mathbb{B}(\mathcal{H})$ with $\left(\rho_{1} \otimes \mathrm{id}_{A}\right) \tilde{\mathcal{V}}^{A}=\mathrm{U}^{\mathcal{H}}$ because $\mathrm{U}^{\mathcal{H}}$ is a corepresentation. Similarly, there is a unique representation $\rho_{2}: \hat{B}^{\mathrm{u}} \rightarrow \mathbb{B}(\mathcal{K})$ with $\left(\rho_{2} \otimes \mathrm{id}_{B}\right) \tilde{\mathcal{V}}^{B}=\mathrm{U}^{\mathcal{K}}$.

Proposition 3.16 shows that any bicharacter $\chi \in \mathcal{U}(\hat{A} \otimes \hat{B})$ lifts uniquely to a bicharacter $\chi^{\mathrm{u}} \in \mathcal{U}\left(\hat{A}^{\mathrm{u}} \otimes \hat{B}^{\mathrm{u}}\right)$. We claim that

$$
Z:=\left(\rho_{1} \otimes \rho_{2}\right)\left(\chi^{\mathrm{u}}\right)^{*} \in \mathbb{B}(\mathcal{H} \otimes \mathcal{K})
$$

verifies 4.14 for any $\chi$-Heisenberg pair $(\alpha, \beta)$. (Our formulation of Theorem 4.23 highlights the property of the operator $\left(\rho_{1} \otimes \rho_{2}\right)\left(\chi^{\mathrm{u}}\right)^{*}$ that is crucial for the proof of Theorem 4.24 , and it avoids universal quantum groups.)

We will actually prove

$$
\tilde{\mathcal{V}}_{1 \alpha}^{A} \tilde{\mathcal{V}}_{2 \beta}^{B}=\tilde{\mathcal{V}}_{2 \beta}^{B} \tilde{\mathcal{V}}_{1 \alpha}^{A} \chi_{12}^{\mathrm{u}} \quad \text { in } \mathcal{U}\left(\hat{A}^{\mathrm{u}} \otimes \hat{B}^{\mathrm{u}} \otimes \mathbb{K}(\mathcal{L})\right)
$$

for any $\chi$-Heisenberg pair $(\alpha, \beta)$. Applying $\rho_{1}$ and $\rho_{2}$ to the first two legs then gives (4.14 because $\left(\rho_{1} \otimes \operatorname{id}_{A}\right) \tilde{\mathcal{V}}^{A}=\mathrm{U}^{\mathcal{H}}$ and $\left(\rho_{2} \otimes \operatorname{id}_{B}\right) \tilde{\mathcal{V}}^{B}=\mathrm{U}^{\mathcal{K}}$.

When we apply the reducing morphisms $\hat{\Lambda}_{A}: \hat{A}^{\mathrm{u}} \rightarrow \hat{A}$ and $\hat{\Lambda}_{B}: \hat{B}^{\mathrm{u}} \rightarrow \hat{B}$ to the first two legs in (4.16), we get $\mathrm{W}_{1 \alpha}^{A} \mathrm{~W}_{2 \beta}^{B}=\mathrm{W}_{2 \beta}^{B} \mathrm{~W}_{1 \alpha}^{A} \chi_{12}$, which is exactly the definition of a Heisenberg pair (see Definition 4.5). A routine computation, using 2.35, shows that

$$
T:=\left(\tilde{\mathcal{V}}_{1 \alpha}^{A}\right)^{*}\left(\tilde{\mathcal{V}}_{2 \beta}^{B}\right)^{*} \tilde{\mathcal{V}}_{1 \alpha}^{A} \tilde{\mathcal{V}}_{2 \beta}^{B} \in \mathcal{U}\left(\hat{A}^{\mathrm{u}} \otimes \hat{B}^{\mathrm{u}} \otimes \mathbb{K}(\mathcal{L})\right)
$$

is a character in the first two legs, that is, $\left(\hat{\Delta}_{A^{\mathrm{u}}} \otimes \operatorname{id}_{\hat{B}^{\mathrm{u}}} \otimes \mathrm{id}_{\mathcal{L}}\right) T=T_{234} T_{134}$ and $\left(\operatorname{id}_{\hat{A}^{\mathrm{u}}} \otimes \hat{\Delta}_{B^{\mathrm{u}}} \otimes \mathrm{id}_{\mathcal{L}}\right) T=$ $T_{124} T_{134}$. Thus $T$ and $\chi_{12}^{\mathrm{u}}$ are two bicharacters in $\mathcal{U}\left(\hat{A}^{\mathrm{u}} \otimes \hat{B}^{\mathrm{u}} \otimes \mathbb{K}(\mathcal{L})\right)$ that both lift the bicharacter $\chi_{12}$ in $\mathcal{U}(\hat{A} \otimes \hat{B} \otimes \mathbb{K}(\mathcal{L}))$. Using Lemma 2.41 twice, we get that any such bicharacter has a unique lifting. Thus $T=\chi_{12}^{\mathrm{u}}$ as asserted. This finishes the proof of Theorem 4.23 .

The Hilbert space representation

$$
\varphi \otimes \psi \otimes \mathrm{id}: A \otimes B \otimes \mathbb{K}(\mathcal{L}) \rightarrow \mathbb{B}(\mathcal{H} \otimes \mathcal{K} \otimes \mathcal{L})
$$

is faithful because $\varphi$ and $\psi$ are faithful. Hence the pair of representations

$$
\begin{aligned}
& (\varphi \otimes \alpha) \gamma_{13}: C \rightarrow \mathbb{B}(\mathcal{H} \otimes \mathcal{K} \otimes \mathcal{L}) \\
& (\psi \otimes \beta) \delta_{23}: D \rightarrow \mathbb{B}(\mathcal{H} \otimes \mathcal{K} \otimes \mathcal{L})
\end{aligned}
$$

of $C$ and $D$ gives a faithful representation of $C \otimes_{\chi} D$; that is, there is a unique faithful representation of $C \nabla_{\chi} D$ that gives the above two representations when composed with $\iota_{C}$ and $\iota_{D}$. 
Lemma 4.26. The pair of representations $\left(\varphi_{1}, \operatorname{Ad}_{z_{12}} \circ \psi_{2}\right)$ of $(C, D)$ on $\mathcal{H} \otimes \mathcal{K} \otimes \mathcal{L}$ is unitarily equivalent to the pair $\left((\varphi \otimes \alpha) \gamma_{13},(\psi \otimes \beta) \delta_{23}\right)$ on the same Hilbert space through conjugation by the unitary $\mathrm{U}_{1 \alpha}^{\mathcal{H}} \mathrm{U}_{2 \beta}^{\mathcal{K}}$.

Proof. We must prove

$$
\begin{aligned}
\mathrm{U}_{1 \alpha}^{\mathcal{H}} \mathrm{U}_{2 \beta}^{\mathcal{K}}\left(\varphi(c) \otimes 1_{\mathcal{K}} \otimes 1_{\mathcal{L}}\right)\left(\mathrm{U}_{2 \beta}^{\mathcal{K}}\right)^{*}\left(\mathrm{U}_{1 \alpha}^{\mathcal{H}}\right)^{*} & =(\varphi \otimes \alpha) \gamma_{13}(c), \\
\mathrm{U}_{1 \alpha}^{\mathcal{H}} \mathrm{U}_{2 \beta}^{\mathcal{K}} Z_{12}\left(1_{\mathcal{H}} \otimes \psi(d) \otimes 1_{\mathcal{L}}\right) Z_{12}^{*}\left(\mathrm{U}_{2 \beta}^{\mathcal{K}}\right)^{*}\left(\mathrm{U}_{1 \alpha}^{\mathcal{H}}\right)^{*} & =(\psi \otimes \beta) \delta_{23}(d)
\end{aligned}
$$

for all $c \in C, d \in D$. To check the first equality, we use first that $\mathrm{U}_{2 \beta}^{\mathcal{K}}$ commutes with $\varphi(c)_{1}$ because both act on different legs, and secondly the covariance condition 4.13 for $\left(\varphi, \mathrm{U}^{\mathcal{H}}\right)$ with $\alpha$ applied to the leg $A$ :

$$
\mathrm{U}_{1 \alpha}^{\mathcal{H}} \mathrm{U}_{2 \beta}^{\mathcal{K}}\left(\varphi(c) \otimes 1_{\mathcal{K}} \otimes 1_{\mathcal{L}}\right)\left(\mathrm{U}_{2 \beta}^{\mathcal{K}}\right)^{*}\left(\mathrm{U}_{1 \alpha}^{\mathcal{H}}\right)^{*}=\mathrm{U}_{1 \alpha}^{\mathcal{H}}\left(\varphi(c) \otimes 1_{\mathcal{K}} \otimes 1_{\mathcal{L}}\right)\left(\mathrm{U}_{1 \alpha}^{\mathcal{H}}\right)^{*}=(\varphi \otimes \alpha) \gamma(c)_{13} .
$$

To check the second equality, we first use 4.14; secondly, that $\mathrm{U}_{1 \alpha}^{\mathcal{H}}$ and $\psi(d)_{2}$ act in different legs to commute them; and thirdly the covariance condition 4.13 for $\left(\psi, \mathrm{U}^{\mathcal{K}}\right)$ with $\beta$ applied to the leg $B$ :

$$
\begin{aligned}
\mathrm{U}_{1 \alpha}^{\mathcal{H}} \mathrm{U}_{2 \beta}^{\mathcal{K}} Z_{12}\left(1_{\mathcal{H}} \otimes \psi(d) \otimes 1_{\mathcal{L}}\right) Z_{12}^{*}\left(\mathrm{U}_{2 \beta}^{\mathcal{K}}\right)^{*}\left(\mathrm{U}_{1 \alpha}^{\mathcal{H}}\right)^{*} & =\mathrm{U}_{2 \beta}^{\mathcal{K}} \mathrm{U}_{1 \alpha}^{\mathcal{H}} \psi(d)_{2}\left(\mathrm{U}_{1 \alpha}^{\mathcal{H}}\right)^{*}\left(\mathrm{U}_{2 \beta}^{\mathcal{K}}\right)^{*} \\
& =\mathrm{U}_{2 \beta}^{\mathcal{K}} \psi(d)_{2}\left(\mathrm{U}_{2 \beta}^{\mathcal{K}}\right)^{*}=(\psi \otimes \beta) \delta(d)_{23} .
\end{aligned}
$$

Proof of Theorem 4.24. We remarked above that the pair of representations $\left((\varphi \otimes \alpha) \gamma_{13},(\psi \otimes \beta) \delta_{23}\right)$ generates a faithful representation of $C \nabla_{\chi} D$. Lemma 4.26 shows that this representation is unitarily equivalent to another representation that restricts to $\varphi_{1} \otimes 1_{\mathcal{L}}$ and $\operatorname{Ad}_{Z_{12}} \circ \psi_{2}=\tilde{\psi}_{2} \otimes 1_{\mathcal{L}}$ on $C$ and $D$, respectively. The latter representation is $\rho \otimes 1_{\mathcal{L}}$ for a faithful representation of $C \otimes_{\chi} D$ on $\mathcal{H} \otimes \mathcal{K}$. This is the faithful representation whose existence is asserted in Theorem 4.24 Uniqueness is clear because $C \otimes_{\chi} D=\iota_{C}(C) \cdot \iota_{D}(D)$.

Theorem 4.27. In the notation of Theorem 4.24, the subspace

$$
C \widetilde{\otimes}_{\chi} D:=\varphi_{1}(C) \cdot \tilde{\psi}_{2}(D) \subseteq \mathbb{B}(\mathcal{H} \otimes \mathcal{K})
$$

is a $\mathrm{C}^{*}$-subalgebra and $\left(C \widetilde{\otimes}_{\chi} D, \varphi_{1}, \tilde{\psi}_{2}\right)$ is a crossed product of $C$ and D. Up to equivalence of crossed products, it does not depend on $\left(\varphi, \mathrm{U}^{\mathcal{H}}\right)$ and $\left(\psi, \mathrm{U}^{\mathcal{K}}\right)$.

The crossed product $\left(C \bigotimes_{\chi} D, \iota_{C}, \iota_{D}\right)$ is equivalent to $\left(C \widetilde{\otimes}_{\chi} D, \varphi_{1}, \tilde{\psi}_{2}\right)$ and, up to equivalence of crossed products, does not depend on the Heisenberg pair $(\alpha, \beta)$.

Proof. Since $C \widetilde{\otimes}_{\chi} D=\rho\left(C \otimes_{\chi} D\right)$ and $\rho \circ \iota_{C}=\varphi_{1}, \rho \circ \iota_{D}=\tilde{\psi}_{2}$, by Theorem 4.24. $C \widetilde{\otimes}_{\chi} D$ is a $C^{*}$-algebra, $\left(C \widetilde{\nabla}_{\chi} D, \varphi_{1}, \tilde{\psi}_{2}\right)$ is a crossed product of $C$ and $D$, and it is equivalent to the crossed product $\left(C \otimes_{\chi} D, \iota_{C}, \iota_{D}\right)$.

Since the unitary $Z$ is the same for all Heisenberg pairs $(\alpha, \beta)$, the crossed product $\left(C \widetilde{\nabla}_{\chi} D, \varphi_{1}, \tilde{\psi}_{2}\right)$ does not depend on $(\alpha, \beta)$; hence up to equivalence $\left(C \nabla_{\chi} D, \iota_{C}, \iota_{D}\right)$ does not depend on $(\alpha, \beta)$. And since $\left(C \nabla_{\chi} D, \iota_{C}, \iota_{D}\right)$ does not depend on $\left(\varphi, \mathrm{U}^{\mathcal{H}}\right)$ and $\left(\psi, \mathrm{U}^{\mathcal{K}}\right)$, neither does $\left(C \widetilde{\nabla}_{\chi} D, \varphi_{1}, \tilde{\psi}_{2}\right)$, up to equivalence.

As a special case of Theorem 4.24 the usual spatial tensor product $C \otimes D$ does not depend on the chosen faithful representations of $C$ and $D$. But we do not reprove this classical result. Rather, we reduce analogous statements for noncommutative tensor products to this case by embedding the latter into commutative tensor products with more factors.

\subsection{Properties of the twisted tensor product}

In this section, we establish functoriality of the twisted tensor product and a result about cocycle conjugacy.

We begin with an easy symmetry property:

Proposition 4.28. The crossed products $\left(C \nabla_{\chi} D, \iota_{C}, \iota_{D}\right)$ and $\left(D \otimes_{\hat{\chi}} C, \iota_{D}^{\prime}, \iota_{C}^{\prime}\right)$ are canonically isomorphic. 
Proof. Let $\left(\mathrm{U}^{\mathcal{H}}, \varphi\right)$ and $\left(\mathrm{U}^{\mathcal{K}}, \psi\right)$ be faithful covariant representations of $C$ and $D$ on Hilbert spaces $\mathcal{H}$ and $\mathcal{K}$, respectively. Theorem 4.27 yields

$$
\begin{aligned}
& \left(C \nabla_{\chi} D, \iota_{C}, \iota_{C}\right) \cong\left(C \widetilde{\otimes}_{\chi} D, \varphi_{1}, \tilde{\psi}_{2}\right), \\
& \left(D \otimes_{\hat{\chi}} C, \iota_{D}^{\prime}, \iota_{C}^{\prime}\right) \cong\left(D \widetilde{\nabla}_{\hat{\chi}} C, \psi_{1}, \tilde{\varphi}_{2}\right)
\end{aligned}
$$

with $C \widetilde{\nabla}_{\chi} D \subseteq \mathbb{B}(\mathcal{H} \otimes \mathcal{K}), D \widetilde{\otimes}_{\hat{\chi}} C \subseteq \mathbb{B}(\mathcal{K} \otimes \mathcal{H})$; here $\psi_{1}(d):=\left(\psi(d) \otimes 1_{\mathcal{H}}\right)$ and $\tilde{\varphi}_{2}(c):=\hat{Z}\left(1_{\mathcal{K}} \otimes \psi(c)\right) \hat{Z}^{*}$, where $Z$ satisfies (4.14) and $\hat{Z}=\Sigma Z^{*} \Sigma$. The pair of representations $\left(\varphi_{1}, \tilde{\psi}_{2}\right)$ of $(C, D)$ on $\mathcal{H} \otimes \mathcal{K}$ is unitarily equivalent to the pair of representations $\left(\psi_{1}, \tilde{\varphi}_{2}\right)$ on $\mathcal{K} \otimes \mathcal{H}$ via the unitary $\Sigma Z^{*}$.

\subsubsection{Functoriality for quantum group morphisms}

Let $\mathbb{G}=\left(A, \Delta_{A}\right), \mathbb{H}=\left(B, \Delta_{B}\right), \mathbb{G}_{2}=\left(A_{2}, \Delta_{A_{2}}\right)$ and $\mathbb{H}_{2}=\left(B_{2}, \Delta_{B_{2}}\right)$ be quantum groups. Let $\chi^{\prime} \in$ $\mathcal{U}\left(\hat{A} \otimes A_{2}\right)$ and $\chi^{\prime \prime} \in \mathcal{U}\left(\hat{B} \otimes B_{2}\right)$ bicharacters; hence quantum group homomorphisms $G \rightarrow \mathbb{G}_{2}$ and $\mathbb{H} \rightarrow \mathbb{H}_{2}$, respectively.

Let $\chi_{2} \in \mathcal{U}\left(\hat{A}_{2} \otimes \hat{B}_{2}\right)$ be a bicharacter. We may view $\chi_{2}$ as a quantum group morphism $\mathbb{G}_{2} \rightarrow \widehat{\mathbb{H}}_{2}$. Composing this with the given quantum group morphisms $\chi^{\prime}: \mathbb{G} \rightarrow \mathbb{G}_{2}$ and $\widehat{\chi^{\prime \prime}}: \widehat{\mathbb{H}}_{2} \rightarrow \widehat{\mathbb{H}}$, we get a bicharacter $\chi:=\widehat{\chi^{\prime \prime}} * \chi_{2} * \chi^{\prime} \in \mathcal{U}(\hat{A} \otimes \hat{B})$, which we view as a quantum group homomorphism $\mathbb{G} \rightarrow \mathbb{H}$.

Let $(C, \gamma)$ and $(D, \delta)$ be a $\mathbb{G}-\mathrm{C}^{*}$-algebra and an $\mathbb{H}-\mathrm{C}^{*}$-algebra, respectively. The description of $f$ in Theorem 3.27 is as a functor from the category of $\mathbb{G}_{-} \mathrm{C}^{*}$-algebras to $\mathbb{G}_{2}$ - $\mathrm{C}^{*}$-algebras that does not change the underlying $\mathrm{C}^{*}$-algebra. In particular, this functor maps $\gamma$ to a continuous $\mathbb{G}_{2}$-coaction $\gamma_{2}: C \rightarrow C \otimes A_{2}$ on $C$. Similarly, $g$ maps $\delta$ to a continuous $\mathbb{H}_{2}$-coaction $\delta_{2}: D \rightarrow D \otimes B_{2}$ on $D$.

Theorem 4.29. In the situation above, the crossed products $\left(C, \gamma_{2}\right) \bigotimes_{\chi_{2}}\left(D, \gamma_{2}\right)$ and $(C, \gamma) \bigotimes_{\chi}(D, \gamma)$ of $C$ and $D$ are equivalent.

Proof. Let $\left(\varphi, \mathrm{U}^{\mathcal{H}}\right)$ be a $\mathbb{G}$-covariant representation of $(C, \gamma)$ on $\mathcal{H}$ and let $\left(\psi, \mathrm{U}^{\mathcal{K}}\right)$ be an $\mathbb{H}$-covariant representation of $(D, \delta)$ on $\mathcal{K}$.

Proposition 3.31 shows that the quantum group morphism $\chi^{\prime}$ turns $\mathrm{U}^{\mathcal{H}}$ into a corepresentation $U_{2}^{\mathcal{H}}$ of $\mathbb{G}_{2}$ on $\mathcal{H}$. Similarly, $\chi^{\prime \prime}$ turns $\mathrm{U}^{\mathcal{K}}$ into a corepresentation $U_{2}^{\mathcal{K}}$ of $\mathbb{H}_{2}$ on $\mathcal{K}$, and $\left(\psi, U_{2}^{\mathcal{K}}\right)$ is a covariant representation of $\left(D, \delta_{2}\right)$.

The bicharacters $\chi \in \mathcal{U}(\hat{A} \otimes \hat{B})$ and $\chi_{2} \in \mathcal{U}\left(\hat{A}_{2} \otimes \hat{B}_{2}\right)$ lift uniquely to bicharacters $\chi^{\mathrm{u}} \in \mathcal{U}\left(\hat{A}^{\mathrm{u}} \otimes \hat{B}^{\mathrm{u}}\right)$ and $\chi_{2}^{\mathrm{u}} \in \mathcal{U}\left(\hat{A}_{2}^{\mathrm{u}} \otimes \hat{B}_{2}^{\mathrm{u}}\right)$ by Proposition 3.16 Let $\hat{f}^{\mathrm{u}}: \hat{A}_{2}^{\mathrm{u}} \rightarrow \hat{A}^{\mathrm{u}}$ and $\hat{g}^{\mathrm{u}}: \hat{B}_{2}^{\mathrm{u}} \rightarrow \hat{B}^{\mathrm{u}}$ be Hopf ${ }^{*}$-homomorphisms between universal duals equivalent to the bicharacters $\chi^{\prime} \in \mathcal{U}\left(\hat{A} \otimes A_{2}\right)$ and $\chi^{\prime \prime} \in \mathcal{U}\left(\hat{B} \otimes B_{2}\right)$. The bijection between bicharacters and quantum group morphisms is defined in such a way that $\chi^{\mathrm{u}}=\left(\hat{f}^{\mathrm{u}} \otimes \hat{g}^{\mathrm{u}}\right)\left(\chi_{2}^{\mathrm{u}}\right)$. Equation 4.15 then shows that the unitaries $Z$ on $\mathcal{H} \otimes \mathcal{K}$ that are used to construct the twisted tensor products with respect to $\chi$ and $\chi_{2}$ are the same.

Now Theorem 4.24 yields the desired equivalence of crossed products because both are faithfully represented by the same $\mathrm{C}^{*}$-algebra $\varphi(C) \cdot Z \psi(D) Z^{*}$ on $\mathcal{H} \otimes \mathcal{K}$.

The following special cases of Theorem 4.29 are particularly noteworthy.

Example 4.30. Let $\mathbb{G}_{2}=\widehat{\mathbb{H}}, \mathbb{H}_{2}=\mathbb{H}$, let $\chi^{\prime \prime}=\mathrm{W}^{B}: \mathbb{H} \rightarrow \mathbb{H}_{2}$ be the identity quantum group homomorphism and let $\chi^{\prime}=\chi: \mathbb{G} \rightarrow \mathbb{G}_{2}=\widehat{\mathbb{H}}$ viewed as a quantum group morphism as above. Let $\chi_{2}=\widehat{\mathrm{W}}^{B}$ be the reduced bicharacter of $\widehat{\mathbb{H}}$. Then

$$
(C, \gamma) \otimes_{\chi}(D, \delta) \cong\left(C, \gamma_{2}\right) \bigotimes_{\widehat{\mathrm{W}}^{B}}(D, \delta),
$$

where $\gamma_{2}: C \rightarrow C \otimes \hat{B}$ is the $\widehat{\mathbb{H}}$-coaction associated to $\gamma$ by the quantum group morphism $\chi: \mathbb{G} \rightarrow \widehat{\mathbb{H}}$ (see Theorem 3.27).

This is a special case of Theorem 4.29 because the bicharacter $\widehat{\mathrm{W}}^{B}$ describes the identity morphism on the quantum group $\widehat{\mathbb{H}}$. The composition of this with $\chi^{\prime}$ gives again $\chi^{\prime}$, so that the bicharacter $\chi$ that we get from $\chi_{2}=\widehat{\mathrm{W}}^{B}$ by the above construction is indeed the given one. 
Example 4.31. Let $\mathbb{G}_{2}=\mathbb{G}, \mathbb{H}_{2}=\widehat{\mathbb{G}}$, let $\chi^{\prime}=\mathrm{W}^{A}: \mathbb{G}_{2} \rightarrow \mathbb{G}$ be the identity quantum group homomorphism and let $\hat{\chi}: \mathbb{H} \rightarrow \mathbb{H}_{2}=\widehat{\mathbb{G}}$ be the dual of the morphism $\chi: \mathbb{G} \rightarrow \widehat{\mathbb{H}}_{2}$. Let $\chi_{2}=\mathrm{W}^{A}$ be the reduced bicharacter of $\widehat{\mathbb{H}}$. Then

$$
(C, \gamma) \bigotimes_{\chi}(D, \delta) \cong(C, \gamma) \bigotimes_{\mathrm{W}^{A}}\left(D, \delta_{2}\right),
$$

where $\delta_{2}: D \rightarrow D \otimes \hat{A}$ is the $\widehat{\mathbb{G}}$-coaction associated to $\delta$ by the quantum group morphism $\hat{\chi}: \mathbb{H} \rightarrow \widehat{\mathbb{G}}$ (see Theorem 3.27).

The last example reduces the twisted tensor product $\nabla_{\chi}$ for an arbitrary bicharacter to the special case $\mathbb{H}=\widehat{\mathbb{G}}$ and $\chi=\mathrm{W}^{A}$.

\subsubsection{Functoriality for equivariant morphisms}

It is well known that the minimal tensor product is functorial for nondegenerate ${ }^{*}$-homomorphisms; that is, $f \in \operatorname{Mor}\left(C_{1}, C_{2}\right)$ and $g \in \operatorname{Mor}\left(D_{1}, D_{2}\right)$ induce $f \otimes g \in \operatorname{Mor}\left(C_{1} \otimes D_{1}, C_{2} \otimes D_{2}\right)$, which is determined by $(f \otimes g)(c \otimes d):=f(c) \otimes g(d)$. We claim the same for the tensor product $\nabla_{\chi}$ in the following sense:

Lemma 4.32. Let $f:\left(C_{1}, \gamma_{1}\right) \rightarrow\left(C_{2}, \gamma_{2}\right)$ be a $\mathbb{G}$-equivariant morphism and $g:\left(D_{1}, \delta_{1}\right) \rightarrow\left(D_{2}, \delta_{2}\right)$ an $\mathbb{H}$-equivariant morphism. Then there is a unique morphism

$$
f \otimes_{\chi} g: C_{1} \otimes_{\chi} D_{1} \rightarrow C_{2} \otimes_{\chi} D_{2}, \quad \iota_{C_{1}}(c) \cdot \iota_{D_{1}}(d) \mapsto \iota_{C_{2}}(f(c)) \cdot \iota_{D_{2}}(g(d)) .
$$

Proof. The uniqueness and hence the functoriality is clear because the linear span of $\iota_{C_{1}}(c) \cdot \iota_{D_{1}}(d)$ with $c \in C_{1}, d \in D_{1}$ is dense in $C_{1} \otimes_{\chi} D_{1}$.

Let $(\alpha, \beta)$ be a $\chi$-Heisenebrg pair on $\mathcal{H}$. We remarked above that ordinary minimal $\mathrm{C}^{*}$-tensor products are functorial for morphisms, that is, there is a well-defined morphism

$$
f \otimes g \otimes \operatorname{id}_{\mathbb{K}(\mathcal{H})}: C_{1} \otimes D_{1} \otimes \mathbb{K}(\mathcal{H}) \rightarrow C_{2} \otimes D_{2} \otimes \mathbb{K}(\mathcal{H}) .
$$

Lemma 4.18 implies $C_{i} \otimes_{\chi} D_{i} \subseteq \mathcal{M}\left(C_{i} \otimes D_{i} \otimes \mathbb{K}(\mathcal{H})\right)$ for $i=1,2$.

The morphism $f \otimes g \otimes \operatorname{id}_{\mathbb{K}(\mathcal{H})}$ extends to a ${ }^{*}$-homomorphism between multiplier algebras. Since $f$ and $g$ are equivariant, this canonical extension maps $\gamma_{1}(c)_{1 \alpha} \mapsto \gamma_{2}(f(c))_{1 \alpha}$ and $\delta_{1}(d)_{2 \beta} \mapsto \delta_{2}(g(d))_{2 \beta}$. Hence it maps $\iota_{C_{1}}(c) \cdot \iota_{D_{1}}(d)$ to $\iota_{C_{2}}(f(c)) \cdot \iota_{D_{2}}(g(d))$ as needed.

Proposition 4.33. If $f$ and $g$ are injective morphisms, then so is $f \otimes_{\chi} g$, and vice versa.

If $f$ and $g$ are surjective morphisms, then so is $f \nabla_{\chi} g$, and vice versa.

The morphism $f \otimes_{\chi} g$ is invertible if and only if both $f$ and $g$ are invertible.

Proof. If $f$ and $g$ are injective, so is $f \otimes g \otimes \mathrm{id}_{\mathbb{K}(\mathcal{H})}$; hence its extension to multipliers is injective, and so is the restriction to $C_{1} \otimes_{\chi} D_{1}$. Conversely, $\left(f \nabla_{\chi} g\right)\left(\iota_{C_{1}}(c) \iota_{D_{1}}(d)\right)$ vanishes if $f(c)=0$ or $g(d)=0$; hence $f$ and $g$ are injective if $f \nabla_{\chi} g$ is.

If $f$ and $g$ are surjective, then elements of the form $\left(f \nabla_{\chi} g\right)\left(\iota_{C_{1}}(c) \iota_{D_{1}}(d)\right)=\iota_{C_{2}}(f(c)) \iota_{D_{2}}(g(d))$ are linearly dense in $C_{2} \nabla_{\chi} D_{2}$. Hence $f \nabla_{\chi} g$ is surjective as well. Conversely, suppose that $f \nabla_{\chi} g$ is surjective. Then

$$
\iota_{C_{2}}\left(f\left(C_{1}\right)\right) \cdot \iota_{D_{2}}\left(g\left(D_{1}\right)\right) \cdot \mathbb{K}(\mathcal{H})_{3}=\left(C_{2} \otimes_{\chi} D_{2}\right) \cdot \mathbb{K}(\mathcal{H})_{3}=C_{2} \otimes D_{2} \otimes \mathbb{K}(\mathcal{H})
$$

by Lemma 4.18 We also have $\iota_{C_{2}}\left(f\left(C_{1}\right)\right) \iota_{D_{2}}\left(g\left(D_{1}\right)\right) \cdot \mathbb{K}(\mathcal{H})_{3} \subseteq f\left(C_{1}\right) \otimes g\left(D_{1}\right) \otimes \mathbb{K}(\mathcal{H})$. Applying slice maps to $C_{2}$ and $D_{2}$, we get $f\left(C_{1}\right)=C_{2}$ and $g\left(D_{1}\right)=D_{2}$.

We now use Proposition 4.33 for the equivariant embeddings $\gamma: C \rightarrow C \otimes A$ and $\delta: D \rightarrow D \otimes B$ provided in Lemma 2.34 to get an embedding

$$
(C, \gamma) \otimes_{\chi}(D, \delta) \rightarrow C \otimes D \otimes(A, \Delta) \otimes_{\chi}(B, \Delta) .
$$

Thus we may describe $(C, \gamma) \otimes_{\chi}(D, \delta)$ as the crossed product generated by the embeddings $\left(\right.$ id $\left.\otimes \iota_{A}\right) \gamma_{13}$ of $C$ and $\left(\mathrm{id} \otimes \iota_{B}\right) \delta_{23}$ of $D$ into $C \otimes D \otimes(A, \Delta) \otimes_{\chi}(B, \Delta)$. This description is particularly useful if we know $(A, \Delta) \otimes_{\chi}(B, \Delta)$ more explicitly. 


\subsubsection{Cocycle conjugacy}

We now consider the special case where the $\mathbb{G}$-coaction is changed to an equivalent one by a cocycle:

Definition 4.34 (2, Définition 0.4]). A $\gamma$-cocycle is a unitary $u \in \mathcal{M}(C \otimes A)$ with

$$
u_{12}\left(\gamma \otimes \operatorname{id}_{A}\right) u=\left(\operatorname{id}_{C} \otimes \Delta_{A}\right) u \quad \text { in } \mathcal{U}(C \otimes A \otimes A) .
$$

We only treat cocycles that satisfy an extra Podleś condition:

Lemma 4.35. Let $u \in \mathcal{U}(C \otimes A)$ be a $\gamma$-cocycle. Define a morphism $\gamma_{u}:=\operatorname{Ad}_{u} \circ \gamma: C \rightarrow C \otimes A$. This is a continuous coaction of $\mathbb{G}$ if and only if

$$
\gamma(C) \cdot u^{*} \cdot\left(1_{C} \otimes A\right)=C \otimes A .
$$

Proof. The morphism $\gamma_{u}$ is faithful because $\gamma$ is. We check that it is a comodule structure:

$$
\left(\operatorname{id}_{C} \otimes \Delta_{A}\right) \gamma_{u}(c)=\left(\operatorname{id}_{C} \otimes \Delta_{A}\right)\left(u \gamma(c) u^{*}\right)=u_{12}\left(\left(\gamma \otimes \operatorname{id}_{A}\right)(u \gamma(c)) u^{*}\right) u_{12}^{*}=\left(\gamma_{u} \otimes \operatorname{id}_{A}\right) \gamma_{u}(c)
$$

for all $c \in C$; the first equality follows from the definition of $\gamma_{u}$, the second equality uses 4.17) and 2.22 for $\gamma$; the third equality again uses 4.17 for all $c \in C$.

Since $u \in \mathcal{U}(C \otimes A)$ we have $u(C \otimes A)=C \otimes A$. Hence 4.18$)$ is equivalent to the Podleś condition $u \gamma(C) u^{*} \cdot(1 \otimes A)=C \otimes A$ for $\gamma_{u}$.

The following result generalises [2, Proposition 7.6].

Theorem 4.36. Let $u$ be $a \gamma$-cocycle and let $v$ be a $\delta$-cocycle. Assume both satisfy the Podleś condition 4.18. Define the coactions $\gamma_{u}$ and $\delta_{v}$ as above. Then

$$
(C, \gamma) \otimes_{\chi}(D, \delta) \cong\left(C, \gamma_{u}\right) \bigotimes_{\chi}\left(D, \delta_{v}\right)
$$

This isomorphism is not one of crossed products, that is, it is not compatible with the embeddings of $C$ and $D$.

Proof. We describe the isomorphism above more explicitly. To simplify notation, we treat only $u$ and assume $v=1$. We define a $\mathbb{G}$-coaction on $\mathbb{M}_{2}(C)$ by

$$
\left(\begin{array}{ll}
c_{11} & c_{12} \\
c_{21} & c_{22}
\end{array}\right) \mapsto\left(\begin{array}{cc}
\gamma\left(c_{11}\right) & \gamma\left(c_{12}\right) u^{*} \\
u \gamma\left(c_{21}\right) & u \gamma\left(c_{22}\right) u^{*}
\end{array}\right)
$$

The upper left and lower right corners are $(C, \gamma)$ and $\left(C, \gamma_{u}\right)$, respectively. Thus $(C, \gamma) \nabla_{\chi}(D, \delta)$ and $\left(C, \gamma_{u}\right) \otimes_{\chi}(D, \delta)$ are subalgebras of $\mathbb{M}_{2}(C) \otimes D$.

Conjugation by the partial isometry $s=\left(\begin{array}{ll}0 & 0 \\ 1 & 0\end{array}\right)$ and its adjoint gives isomorphisms between the two corners $C \subseteq \mathbb{M}_{2}(C)$. The strictly continuous extension of $\iota_{\mathbb{M}_{2}(C)}$ maps $s$ to a partial isometry in $\mathbb{M}_{2}(C) \otimes_{\chi} D$. Conjugation by this partial isometry and its adjoint restricts to isomorphisms between $(C, \gamma) \otimes_{\chi}(D, \delta)$ and $\left(C, \gamma_{u}\right) \nabla_{\chi}(D, \delta)$

Definition 4.37. We call a continuous coaction inner if it is a cocycle-twist of the trivial coaction.

Corollary 4.38. The crossed product $(C, \gamma) \bigotimes_{\chi}(D, \delta)$ is isomorphic to $C \otimes D$ if $\gamma$ or $\delta$ is inner.

Proof. Let $u \in \mathcal{M}(C \otimes A)$ be a cocycle for the trivial coaction $\tau(c):=c \otimes 1$ and let $\gamma=\tau_{u}$. The cocycle $u$ satisfies 4.18 by Lemma 4.35 Now Theorem 4.36 and Example 4.19 give $(C, \gamma) \otimes_{\chi} D \cong(C, \tau) \otimes_{\chi} D \cong C \otimes D$. A similar proof works if $\delta$ is inner.

Example 4.39. Let $\mathrm{U}^{\mathcal{H}}$ and $\mathrm{U}^{\mathcal{K}}$ be corepresentations of $A$ and $B$ on Hilbert spaces $\mathcal{H}$ and $\mathcal{K}$. These are cocycles for the trivial coaction on $\mathbb{K}(\mathcal{H})$. Assume 4.18 to get continuous coactions on $\mathbb{K}(\mathcal{H})$ and $\mathbb{K}(\mathcal{K})$. Then

$$
\mathbb{K}(\mathcal{H}) \bigotimes_{\chi} \mathbb{K}(\mathcal{K}) \cong \mathbb{K}(\mathcal{H}) \otimes \mathbb{K}(\mathcal{K}) \cong \mathbb{K}(\mathcal{H} \otimes \mathcal{K}) .
$$

This explains the Hilbert space realisation of $C \otimes_{\chi} D$ in Theorem 4.24 in the case where the corepresentations $\mathrm{U}^{\mathcal{H}}$ and $\mathrm{U}^{\mathcal{K}}$ used there satisfy the technical condition 4.18). Then we get a faithful morphism $C \otimes_{\chi} D \rightarrow \mathbb{K}(\mathcal{H}) \bigotimes_{\chi} \mathbb{K}(\mathcal{K})$ from Proposition 4.33. When we identify $\mathbb{K}(\mathcal{H}) \bigotimes_{\chi} \mathbb{K}(\mathcal{K}) \cong \mathbb{K}(\mathcal{H} \otimes \mathcal{K})$ as above, we get a faithful representation of $C \nabla_{\chi} D$ on $\mathcal{H} \otimes \mathcal{K}$. 


\subsection{Examples of twisted tensor products}

We show in Section 4.5.1 that the skew-commutative tensor product of $\mathbb{Z} / 2$-graded $\mathrm{C}^{*}$-algebras is a special case of our theory.

In Section 4.5.2, we treat crossed products for coactions and construct the dual coaction on a crossed product using the functoriality of $\otimes_{\chi}$.

\subsubsection{Skew-commutative tensor products}

Let $\mathbb{Z} / 2=\{0,1\}$ be the two-element group. Let $\mathbb{G}=\mathbb{H}$ be $\mathrm{C}^{*}(\mathbb{Z} / 2)$ with the usual comultiplication. Thus a $\mathbb{G}$-coaction on a $\mathrm{C}^{*}$-algebra $C$ is a $\mathbb{Z} / 2$-grading: a decomposition $C=C_{0} \oplus C_{1}$ into involutive, closed, linear subspaces $C_{0}$ and $C_{1}$ of even and odd elements such that

$$
C_{i} \cdot C_{j}=C_{i+j \bmod 2,} C_{i}^{*}=C_{i} .
$$

Equivalently, $\alpha^{\prime}\left(c_{0}+c_{1}\right):=c_{0}-c_{1}$ for $c_{i} \in C_{i}$ defines an involutive ${ }^{*}$-automorphism of $C$.

The skew-commutative tensor product of two $\mathbb{Z} / 2$-graded $\mathrm{C}^{*}$-algebras $C$ and $D$ is defined in $[18, \S 2.6]$ by imposing the commutation relation that $c \in C$ and $d \in D$ anti-commute if both are odd, and commute if one of them is even. This leads to the ${ }^{*}$-algebra structure

$$
\begin{aligned}
\left(c_{1} \hat{\odot} d_{1}\right) \cdot\left(c_{2} \hat{\odot} d_{2}\right) & :=(-1)^{\operatorname{deg}\left(c_{2}\right) \cdot \operatorname{deg}\left(d_{1}\right)} c_{1} c_{2} \hat{\odot} d_{1} d_{2}, \\
(c \hat{\odot} d)^{*}: & =(-1)^{\operatorname{deg}(c) \cdot \operatorname{deg}(d)} c^{*} \hat{\odot} d^{*}
\end{aligned}
$$

on the algebraic tensor product $C \hat{\odot} D$ of $C$ and $D$. The skew-commutative $\mathrm{C}^{*}$-tensor product $C \hat{\otimes} D$ is the completion of the ${ }^{*}$-algebra $C \hat{\odot} D$ in the $\mathrm{C}^{*}$-norm

$$
\|x\|:=\sup \frac{(\rho \hat{\otimes} \lambda)\left(y^{*} \cdot x^{*} \cdot x \cdot y\right)}{(\rho \hat{\otimes} \lambda)\left(y^{*} \cdot y\right)}
$$

over all non-zero elements $y \in C \hat{\odot} D$ and all even states $\rho \in C^{*}$ and $\lambda \in D^{*}$; here the products and adjoints are with respect to the ${ }^{*}$-algebra structure on $C \hat{\odot} D$.

The obvious formulas define morphisms $\iota_{C}: C \rightarrow C \hat{\otimes} D$ and $\iota_{D}: D \rightarrow C \hat{\otimes} D$, so that $C \hat{\otimes} D$ is a crossed product of $C$ and $D$. We want to show that $C \hat{\otimes} D \cong C \otimes_{\chi} D$ for a suitable bicharacter $\chi \in \mathcal{U}(\hat{A} \otimes \hat{A})$.

The dual $\widehat{\mathbb{G}}$ is the group $\mathbb{Z} / 2$, so that $\hat{A} \otimes \hat{B} \cong \mathrm{C}(\mathbb{Z} / 2 \times \mathbb{Z} / 2)$ and a bicharacter $\chi$ is a bicharacter $\mathbb{Z} / 2 \times \mathbb{Z} / 2 \rightarrow \mathbb{T}$ in a more classical sense. The unique non-trivial bicharacter is defined by $\chi(1,1)=-1$ and $\chi(i, j)=1$ if $i=0$ or $j=0$.

Theorem 4.40. Let $C$ and $D$ be $\mathbb{Z} / 2$-graded $\mathrm{C}^{*}$-algebras and let $\chi$ be the non-trivial bicharacter in $\mathrm{C}(\mathbb{Z} / 2 \times \mathbb{Z} / 2)$. Then the crossed product $\left(C \bigotimes_{\chi} D, \iota_{C}, \iota_{D}\right)$ of $C$ and $D$ is naturally isomorphic to their skew-commutative tensor product.

Proof. A covariant representation of $C$ is given by a $\mathbb{Z} / 2$-graded Hilbert space $\mathcal{H}=\mathcal{H}_{0} \oplus \mathcal{H}_{1}$ and a representation $\varphi: C \rightarrow \mathbb{B}(\mathcal{H})$ with $\varphi\left(c_{i}\right)\left(\mathcal{H}_{j}\right) \subseteq \mathcal{H}_{i+j}$ for all $i, j \in \mathbb{Z} / 2$. We choose such a faithful covariant representation of $A$ and a faithful covariant representation $\psi: D \rightarrow \mathbb{B}(\mathcal{K})$ on a $\mathbb{Z} / 2$-graded Hilbert space $\mathcal{K}=\mathcal{K}_{0} \oplus \mathcal{K}_{1}$

Since $\hat{A}^{\mathrm{u}}=\hat{A}$, the unitary $Z$ that is used in the Hilbert space description of $C \nabla_{\chi} D$ is described most easily by 4.15. This gives $Z(\xi \otimes \eta)=-\xi \otimes \eta$ if $\xi \in \mathcal{H}_{1}$ and $\eta \in \mathcal{K}_{1}$, and $Z(\xi \otimes \eta)=\xi \otimes \eta$ if $\xi \in \mathcal{H}_{0}$ or $\eta \in \mathcal{K}_{0}$. Thus $\Sigma Z: \mathcal{H} \otimes \mathcal{K} \rightarrow \mathcal{K} \otimes \mathcal{H}$ is the braiding operator from the Koszul sign rule. The representations $\varphi_{1}$ and $\tilde{\psi}_{2}$ in Theorem 4.24 are

$$
\varphi_{1}(c)(\xi \otimes \eta)=(\varphi(c) \xi) \otimes \eta, \quad \tilde{\psi}_{2}(d)(\xi \otimes \eta)=(-1)^{\operatorname{deg}(d) \operatorname{deg}(\xi)} \xi \otimes \psi(d) \eta,
$$

as expected from the Koszul sign rule. It remains to show that this pair of representations of $C$ and $D$ yields a faithful representation of the skew-commutative tensor product $C \hat{\otimes} D$. It is clear that we get a ${ }^{*}$-representation of $C \hat{\odot} D$.

We must show that, for any $x \in C \hat{\odot} D$, its operator norm on $\mathcal{H} \otimes \mathcal{K}$ is equal to the norm defined in 4.19. The GNS-representation for an even state $\rho: C \rightarrow \mathbb{C}$ on the Hilbert space $L^{2}(C, \rho)$ is a 
covariant representation if we let $L^{2}(C, \rho)_{i}$ be the closure of $C_{i}$ in $L^{2}(C, \rho)$. The direct sum of these GNS-representations for all even states is a faithful representation of $C$ because any state on $C_{0}$ extends to a state on $C$ and a representation of $C$ is faithful once it is faithful on $C_{0}$. Since $C \nabla_{\chi} D$ does not depend on the covariant representations, we may assume that $\varphi$ and $\psi$ are these direct sums of covariant GNS-representations of $C$ and $D$, respectively. The resulting representations $\varphi_{1}$ and $\tilde{\psi}_{2}$ are block diagonal with respect to the direct sum over the even states $\rho$ and $\lambda$, and each block is obtained from the GNSrepresentation for the pair of even states $\rho$ and $\lambda$. The elements $y \in C \odot D$ in $(4.19)$ form a dense subset of the Hilbert space $L^{2}(C, \rho) \otimes L^{2}(D, \lambda)$, and the expression in 4.19 for fixed $\rho$ and $\lambda$ is precisely the norm quotient $\|x \cdot y\| /\|y\|$, where $x \cdot y$ is defined using $\varphi_{1} \odot \tilde{\psi}_{2}$. Hence the norm in 4.19 is exactly the operator norm for a particular choice of the covariant representations $\varphi$ and $\psi$.

\subsubsection{Crossed products}

Consider the special case where $\mathbb{H}=\widehat{\mathbb{G}}, \chi=\mathrm{W}^{A} \in \mathcal{U}(\hat{A} \otimes A), D=\hat{A}, \delta=\hat{\Delta}_{A}: \hat{A} \rightarrow \hat{A} \otimes \hat{A}$. We claim that $(C, \gamma) \bigotimes_{\mathrm{W} A}\left(\hat{A}, \hat{\Delta}_{A}\right)$ is the reduced crossed product of $(C, \gamma)$. More precisely, the reduced crossed product $C \rtimes_{\mathrm{r}} \hat{A}$ comes equipped with canonical morphisms $\iota_{C}: C \rightarrow C \rtimes_{\mathrm{r}} \hat{A}$ and $\iota_{\hat{A}}: \hat{A} \rightarrow C \rtimes_{\mathrm{r}} \hat{A}$, such that $\left(C \rtimes_{\mathrm{r}} \hat{A}, \iota_{C}, \iota_{\hat{A}}\right)$ is a crossed product in the sense of Definition 4.1 We claim that this is equivalent to $(C, \gamma) \otimes_{\mathrm{W}^{A}}\left(\hat{A}, \hat{\Delta}_{A}\right)$ as a crossed product.

Let $(\pi, \hat{\pi})$ be the $\mathbb{G}$-Heisenberg pair on the Hilbert space $\mathcal{H}$ constructed in Example 2.18 that is, $\mathrm{W}_{\hat{\pi} \pi}=\mathbb{W}$ is a multiplicative unitary generating $\mathbb{G}$.

Theorem 4.41. There is a faithful morphism $\rho:(C, \gamma) \bigotimes_{\mathrm{W}^{A}}\left(\hat{A}, \hat{\Delta}_{A}\right) \rightarrow C \otimes \mathbb{K}(\mathcal{H})$ with $\rho \circ \iota_{C}=\gamma_{1 \pi}$ and $\rho \circ \iota_{\hat{A}}=\hat{\pi}_{2}$, where

$$
\begin{aligned}
\gamma_{1 \pi}: C & \rightarrow \mathcal{M}(C \otimes \mathbb{K}(\mathcal{H})), & & c \mapsto\left(\operatorname{id}_{C} \otimes \pi\right) \gamma(c), \\
\hat{\pi}_{2}: \hat{A} & \rightarrow \mathcal{M}(C \otimes \mathbb{K}(\mathcal{H})), & \hat{a} & \mapsto 1_{C} \otimes \hat{\pi}(\hat{a}) .
\end{aligned}
$$

Reduced crossed products for locally compact quantum groups (with Haar weights) are defined using the pair of representations $\gamma_{1 \pi}$ and $\hat{\pi}_{2}$ above, where $\pi$ and $\hat{\pi}$ are the regular representations. Thus Theorem 4.41 provides an isomorphism $(C, \gamma) \bigotimes_{\mathrm{W}^{A}}\left(\hat{A}, \hat{\Delta}_{A}\right) \rightarrow C \rtimes_{\mathrm{r}} \hat{A}$ for locally compact quantum groups. For general $\mathrm{C}^{*}$-quantum groups, we may now define the reduced crossed product using the pair of representations $\gamma_{1 \pi}$ and $\hat{\pi}_{2}$ and get the isomorphism $(C, \gamma) \bigotimes_{\mathrm{W}^{A}}\left(\hat{A}, \hat{\Delta}_{A}\right) \rightarrow C \rtimes_{\mathrm{r}} \hat{A}$ in complete generality. The theorem shows that the crossed product does not depend on the choice of $\mathbb{W}$.

Proof of Theorem 4.41. Since

$$
\gamma_{1 \pi} \otimes \operatorname{id}_{\mathbb{K}(\mathcal{H})}: C \otimes \mathbb{K}(\mathcal{H}) \rightarrow C \otimes \mathbb{K}(\mathcal{H}) \otimes \mathbb{K}(\mathcal{H})
$$

is a faithful morphism, the pair of representations $\left(\gamma_{1 \pi}, \hat{\pi}_{2}\right)$ generates a faithful representation of $C \otimes \hat{A}$ if and only the pair $\left(\left(\gamma_{1 \pi} \otimes \operatorname{id}_{\mathbb{K}(\mathcal{H})}\right) \circ \gamma_{1 \pi},\left(\gamma_{1 \pi} \otimes \operatorname{id}_{\mathbb{K}(\mathcal{H})}\right) \circ \hat{\pi}_{2}\right)$ does so. We have $\left(\gamma_{1 \pi} \otimes \operatorname{id}_{\mathbb{K}(\mathcal{H})}\right) \circ \hat{\pi}_{2}(\hat{a})=\hat{\pi}_{3}(\hat{a})$ and

$$
\left(\gamma_{1 \pi} \otimes \operatorname{id}_{\mathbb{K}(\mathcal{H})}\right) \gamma_{1 \pi}(c)=\left(\gamma \otimes \operatorname{id}_{A}\right) \gamma(c)_{1 \pi \pi}=\left(\operatorname{id}_{C} \otimes \Delta_{A}\right) \gamma(c)_{1 \pi \pi}=\mathbb{W}(\mathrm{id} \otimes \pi) \gamma(c)_{12} \mathbb{W}^{*} .
$$

Let $\Sigma_{23}$ be the coordinate flip. Conjugating both representations by the same unitary $\Sigma_{23} \mathbb{W}^{*}$ gives a unitarily equivalent pair of representations. Hence we may further replace $\gamma_{1 \pi}$ and $\hat{\pi}_{2}$ by the representations $c \mapsto(\mathrm{id} \otimes \pi) \gamma(c)_{13}$ of $C$ and

$$
\hat{a} \mapsto \Sigma_{23} \mathbb{W}^{*} \hat{\pi}(\hat{a})_{3} \mathbb{W} \Sigma_{23}=\hat{\Delta}(\hat{a})_{\hat{\pi} \hat{\pi}}
$$

of $\hat{A}$; here we use the standard description of $\hat{\Delta}$ in terms of $\mathbb{W}$.

Thus we arrive at the pair of representations $\left(\operatorname{id}_{C} \otimes \hat{\pi} \otimes \operatorname{id}_{\mathbb{K}(\mathcal{H})}\right) \iota_{C}$ and $\left(\operatorname{id}_{C} \otimes \hat{\pi} \otimes \operatorname{id}_{\mathbb{K}(\mathcal{H})}\right) \iota_{\hat{A}}$ in $C \otimes \hat{A} \otimes \mathbb{K}(\mathcal{H})$ with $\iota_{C}=\gamma_{1 \pi}$ and $\iota_{\hat{A}}=\left(\hat{\Delta}_{A}\right)_{2 \hat{\pi}}$. Since $\hat{\pi}$ is faithful, this pair is equivalent to $\left(\iota_{C}, \iota_{\hat{A}}\right)$. Since the latter pair defines the crossed product $C \otimes \hat{A}$, we see that $\left(\gamma_{1 \pi}, \hat{\pi}_{2}\right)$ generates an equivalent crossed product as claimed. 
Viewing the reduced crossed product as a special case of $\otimes$ gives us more freedom because we may also tensor $(C, \gamma)$ with other $\widehat{\mathbb{G}}-\mathrm{C}^{*}$-algebras and use functoriality. We now describe the dual coaction in this way, using the functoriality of $\otimes$.

The map $\hat{\Delta}: \hat{A} \rightarrow \hat{A} \otimes \hat{A}$ is $\widehat{\mathbb{G}}$-equivariant if $\widehat{\mathbb{G}}$ coacts on $\hat{A} \otimes \hat{A}$ by id $\otimes \hat{\Delta}: \hat{A} \otimes \hat{A} \rightarrow \hat{A} \otimes \hat{A} \otimes \hat{A}$. By the functoriality of $\otimes$, this equivariant morphism induces a morphism

$$
\hat{\delta}: C \rtimes_{r} \hat{A} \cong C \otimes \hat{A} \rightarrow C \otimes(\hat{A} \otimes \hat{A}) \cong \hat{A} \otimes(C \otimes \hat{A}) \cong \hat{A} \otimes\left(C \rtimes_{r} \hat{A}\right) ;
$$

here we use Lemma 4.20 in the second variable to pull out the first factor $\hat{A}$.

Lemma 4.42. The map $\hat{\delta}: C \rtimes_{r} \hat{A} \rightarrow \hat{A} \otimes\left(C \rtimes_{r} \hat{A}\right)$ is a continuous left $\widehat{\mathbb{G}}$-coaction.

Proof. The comodule property of $\hat{\delta}$ follows from the coassociativity of $\hat{\Delta}$ and the functoriality of $\otimes$. The map $\hat{\delta}$ is faithful by Proposition 4.33 The Podleś condition for $\hat{\delta}$ follows because $(\hat{A} \otimes 1) \hat{\Delta}(\hat{A})=\hat{A} \otimes \hat{A}$ : apply $\iota_{\hat{A} \otimes \hat{A}}$ to this equality.

The coaction $\hat{\delta}$ is uniquely determined by the conditions $\hat{\delta}\left(\iota_{C}(c)\right)=1 \otimes \iota_{C}(c)$ and $\hat{\delta}\left(\iota_{\hat{A}}(\hat{a})\right)=\left(\operatorname{id}_{\hat{A}} \otimes \iota_{\hat{A}}\right) \hat{\Delta}$. The same conditions characterise the dual coaction. Thus we have indeed constructed the dual coaction.

The functoriality of $\otimes$ in the first variable gives us the usual functoriality of reduced crossed products.

General tensor products $C \otimes_{\mathrm{W}}(D, \delta)$ are closely related to the crossed product through Lemma 2.34 which shows that $\delta: D \rightarrow D \otimes \hat{A}$ is a $\widehat{\mathbb{G}}$-equivariant embedding for the coaction $\operatorname{id}_{D} \otimes \hat{\Delta}$ on $D \otimes \hat{A}$. By Proposition 4.33 and Lemma 4.20 , this induces a faithful morphism

$$
C \otimes_{\mathrm{W}} D \rightarrow C \otimes_{\mathrm{W}}(D \otimes \hat{A}) \cong D \otimes\left(C \otimes_{\mathrm{W}} \hat{A}\right) \cong D \otimes\left(C \rtimes_{\mathrm{r}} \hat{A}\right)
$$

Now we consider once again the general situation of two quantum groups $\mathbb{G}=\left(A, \Delta_{A}\right)$ and $\mathbb{H}=\left(B, \Delta_{B}\right)$ and a bicharacter $\chi \in \mathcal{U}(\hat{A} \otimes \hat{B})$.

Theorem 4.43. View $\hat{A} \otimes \hat{B}$ as a subalgebra of $\left(C \rtimes_{\mathrm{r}} \hat{A}\right) \otimes\left(D \rtimes_{\mathrm{r}} \hat{B}\right)$ via $\iota_{\hat{A}} \otimes \iota_{\hat{B}}$. The embeddings

$$
\begin{array}{rlrl}
\left(\iota_{C}\right)_{1}: C & \rightarrow\left(C \rtimes_{\mathrm{r}} \hat{A}\right) \otimes\left(D \rtimes_{\mathrm{r}} \hat{B}\right), & & c \mapsto \iota_{C}(c) \otimes 1, \\
\operatorname{Ad}_{\chi^{*}} \circ\left(\iota_{D}\right)_{2}: D \rightarrow\left(C \rtimes_{\mathrm{r}} \hat{A}\right) \otimes\left(D \rtimes_{\mathrm{r}} \hat{B}\right), & & \mapsto \chi^{*}\left(1 \otimes \iota_{D}(d)\right) \chi,
\end{array}
$$

induce a faithful morphism

$$
C \otimes_{\chi} D \rightarrow\left(C \rtimes_{\mathrm{r}} \hat{A}\right) \otimes\left(D \rtimes_{\mathrm{r}} \hat{B}\right) .
$$

Proof. Choose a faithful representation $\varphi_{0}: C \rightarrow \mathbb{B}\left(\mathcal{H}_{0}\right)$. Let $(\pi, \hat{\pi})$ be a $\mathbb{G}-H e i s e n b e r g$ pair as in Example 2.18 acting on a Hilbert space $\mathcal{H}_{\pi}$. Let $\mathcal{H}:=\mathcal{H}_{0} \otimes \mathcal{H}_{\pi}$. Then we get a faithful representation $\varphi_{0} \otimes$ id of $C \otimes \mathbb{K}\left(\mathcal{H}_{\pi}\right)$ on $\mathcal{H}$. This restricts to a faithful representation $\varphi^{\prime}: C \rtimes_{\mathrm{r}} \hat{A} \rightarrow \mathbb{B}(\mathcal{H})$ because the latter is the crossed product generated by the morphisms $\gamma_{1 \pi}: C \rightarrow C \otimes \mathbb{K}\left(\mathcal{H}_{\pi}\right)$ and $\hat{\pi}_{2}: \hat{A} \rightarrow C \otimes \mathbb{K}\left(\mathcal{H}_{\pi}\right)$.

We compare this with the construction of a covariant representation of $(C, \gamma)$ in Example 4.22 . We see that this covariant representation consists of $\rho \circ \iota_{C}: C \rightarrow \mathbb{B}(\mathcal{H})$ and $\mathrm{W}_{\rho \iota^{2}}^{A} \in \mathcal{U}\left(\mathbb{K}\left(\mathcal{H}_{\pi}\right) \otimes A\right)$. Furthermore, the representation of $\hat{A}^{\mathrm{u}}$ used later in the proof of Theorem 4.23 is $\rho \iota_{\hat{A}} \circ \Lambda$ for the reducing morphism $\Lambda: \hat{A}^{\mathrm{u}} \rightarrow \hat{A}$. (Actually, any representation of $C \rtimes_{\mathrm{r}} \hat{A}$ gives a covariant representation of $(C, \gamma)$ in a similar way.)

Now do the same things for $(D, \delta)$ : let $\psi_{0}: D \rightarrow \mathbb{B}\left(\mathcal{K}_{0}\right)$ be a faithful representation; choose an $\mathbb{H}$-Heisenberg pair $(\rho, \hat{\rho})$ as in Example 2.18, acting on a Hilbert space $\mathcal{K}_{\rho} ;$ let $\mathcal{K}:=\mathcal{K}_{0} \otimes \mathcal{K}_{\rho}$; let $\psi^{\prime}$ be the resulting faithful representation of $D \rtimes_{\mathrm{r}} B$ on $\mathcal{K}$; construct a covariant representation of $(D, \delta)$ on $\mathcal{K}$ as in Example 4.22

Theorem 4.24 gives a faithful representation of $C \otimes_{\chi} D$ on $\mathcal{H} \otimes \mathcal{K}$, generated by the representations $\varphi_{1}$ and $\operatorname{Ad}_{Z} \psi_{2}$. By construction, we also get a faithful representation $\varphi^{\prime} \otimes \psi^{\prime}$ of $\left(C \rtimes_{\mathrm{r}} \hat{A}\right) \otimes\left(D \rtimes_{\mathrm{r}} \hat{B}\right)$ on $\mathcal{H} \otimes \mathcal{K}$. The description of $Z$ in 4.15 yields $Z=\left(\varphi^{\prime} \otimes \psi^{\prime}\right)\left(\chi^{*}\right)$. Hence the representations $\varphi_{1}$ and $\operatorname{Ad}_{Z} \psi_{2}$ both factor through the embedding $\varphi^{\prime} \otimes \psi^{\prime}$ and the maps $\left(\iota_{C}\right)_{1}$ and $\operatorname{Ad}_{\chi^{*}} \circ\left(\iota_{D}\right)_{2}$ in the statement of the theorem. We thus get a faithful morphism $C \otimes_{\chi} D \rightarrow\left(C \rtimes_{\mathrm{r}} \hat{A}\right) \otimes\left(D \rtimes_{\mathrm{r}} \hat{B}\right)$ restricting to $\left(\iota_{C}\right)_{1}$ and $\operatorname{Ad}_{\chi *} \circ\left(\iota_{D}\right)_{2}$ on $C$ and $D$, respectively.

For instance, in the situation of Section 4.5.1 this realises the skew-commutative tensor product $C \otimes D$ as a subalgebra of $(C \rtimes \mathbb{Z} / 2) \otimes(D \rtimes \mathbb{Z} / 2)$. 


\subsection{Generalised quantum codouble and Drinfeld double}

In [4, Section 8], Baaj and Vaes have described generalised quantum doubles as a special case of the double crossed product construction, assuming the existence of Haar weights. Let $\mathbb{G}=\left(A, \Delta_{A}\right)$ and $\mathbb{H}=\left(B, \Delta_{B}\right)$ be quantum groups with the reduced bicharacters $\mathrm{W}^{A} \in \mathcal{U}(\hat{A} \otimes A)$ and $\mathrm{W}^{B} \in \mathcal{U}(\hat{B} \otimes B)$, respectively. Let $\chi \in \mathcal{U}(\hat{A} \otimes \hat{B})$ be a bicharacter. Using the $\chi$-Drinfeld commutation relation, we construct a modular multiplicative unitary for generalised Drinfeld doubles. Finally, we show that the generalised quantum codoubles are dual to them.

\subsubsection{Multiplicative unitary}

Starting from a modular multiplicative unitary one can always construct a manageable one (see [38]) giving rise to the same quantum group. Therefore, without loss of generality we assume that $(\pi, \hat{\pi})$ and $(\eta, \hat{\eta})$ are $\mathbb{G}$ - and $\mathbb{H}$-Heisenberg pairs, coming from manageable multiplicative unitaries on the Hilbert spaces $\mathcal{H}$ and $\mathcal{K}$, respectively. Let $(\alpha, \beta)$ be the faithful $\chi$-Heisenberg pair defined in Lemma 4.11 on $\mathcal{L}=\mathcal{H} \otimes \mathcal{K}$. Then $(\bar{\alpha}, \bar{\beta})$, as defined in Example 4.7 , is the associated faithful $\chi$-anti-Heisenberg pair acting on $\overline{\mathcal{L}}$. Let $\hat{\Delta}_{R}: B \rightarrow B \otimes \hat{A}$ be the right quantum group homomorphism associated to the bicharacter $\hat{\chi}$. We define faithful representations of $A, B, \hat{A}, \hat{B}$ on $\mathcal{H}_{\mathcal{D}}:=\overline{\mathcal{L}} \otimes \mathcal{K} \otimes \mathcal{H}$ as:

$$
\begin{array}{ll}
\rho(a):=\left((\bar{\alpha} \otimes \pi) \Delta_{A}(a)\right)_{13} & \text { in } \mathbb{B}\left(\mathcal{H}_{\mathcal{D}}\right) \text { for all } a \in A, \\
\theta(b):=\left(\bar{\beta} \otimes\left((\eta \otimes \hat{\pi}) \hat{\Delta}_{R}\right)\right) \Delta_{B}(b) & \text { in } \mathbb{B}\left(\mathcal{H}_{\mathcal{D}}\right) \text { for all } b \in B, \\
\xi(\hat{a}):=1_{\overline{\mathcal{L}}} \otimes 1_{\mathcal{K}} \otimes \hat{\pi}(\hat{a}) & \text { in } \mathbb{B}\left(\mathcal{H}_{\mathcal{D}}\right) \text { for all } \hat{a} \in \hat{A}, \\
\zeta(\hat{b}):=1_{\overline{\mathcal{L}}} \otimes \hat{\eta}(\hat{b}) \otimes 1_{\mathcal{H}} & \text { in } \mathbb{B}\left(\mathcal{H}_{\mathcal{D}}\right) \text { for all } \hat{b} \in \hat{B},
\end{array}
$$

respectively.

Proposition 4.44. The unitary $\mathbb{W}^{\mathcal{D}} \in \mathcal{U}\left(\mathcal{H}_{\mathcal{D}} \otimes \mathcal{H}_{\mathcal{D}}\right)$ defined by $\mathbb{W}^{\mathcal{D}}:=\widehat{\mathrm{W}}_{\theta \zeta}^{B} \widehat{\mathrm{W}}_{\rho \xi}^{A} \in \mathcal{U}\left(\mathcal{H}_{\mathcal{D}} \otimes \mathcal{H}_{\mathcal{D}}\right)$ is a modular multiplicative unitary.

The proof depends on commutation relations of the representations defined in 4.20 .

Lemma 4.45. Consider the faithful representations on $\mathcal{H}_{\mathcal{D}}$ defined in 4.20 . Then

1. $(\rho, \xi)$ is a $\mathbb{G}$-Heisenberg pair;

2. $(\rho, \theta)$ is a $\chi$-Drinfeld pair;

3. $\theta$ and $\zeta$ commute in the following way:

$$
\widehat{\mathrm{W}}_{\theta 3}^{B} \widehat{\mathrm{W}}_{1 \zeta}^{B}=\widehat{\mathrm{W}}_{1 \zeta}^{B} \cdot \chi_{\xi 3} \cdot \widehat{\mathrm{W}}_{13}^{B} \cdot \chi_{\xi 3}^{*} \cdot \widehat{\mathrm{W}}_{\theta 3}^{B} \quad \text { in } \mathcal{U}\left(B \otimes \mathbb{K}\left(\mathcal{H}_{\mathcal{D}}\right) \otimes \hat{B}\right) ;
$$

4. $\theta$ and $\xi$ commute in the following way:

$$
\widehat{\mathrm{W}}_{\theta 3}^{B} \widehat{\mathrm{W}}_{1 \xi}^{A}=\chi_{\xi 3} \cdot \widehat{\mathrm{W}}_{1 \xi}^{A} \cdot \chi_{\xi 3}^{*} \cdot \widehat{\mathrm{W}}_{\theta 3}^{B} \quad \text { in } \mathcal{U}\left(A \otimes \mathbb{K}\left(\mathcal{H}_{\mathcal{D}}\right) \otimes \hat{B}\right) ;
$$

5. $\rho$ and $\zeta$ commute;

6. $\xi$ and $\zeta$ commute.

Proof. Example 2.23 immediately yields (1). Lemma 4.11 and Proposition 4.14 imply (2).

Using 3.15 we express $\hat{\Delta}_{R}$ in terms of $\hat{\chi}$ in the following way:

$$
\left(\operatorname{id}_{\hat{B}} \otimes \hat{\Delta}_{R}\right) \mathrm{W}^{B}=\mathrm{W}_{12}^{B} \hat{\chi}_{13} \quad \text { in } \mathcal{U}(\hat{B} \otimes B \otimes \hat{A}) .
$$

Applying $\sigma_{23} \sigma_{12}$ on both sides of the last expression and taking adjoints yields:

$$
\left(\hat{\Delta}_{R} \otimes \operatorname{id}_{\hat{B}}\right) \widehat{\mathrm{W}}^{B}=\chi_{23} \widehat{\mathrm{W}}_{13}^{B} \quad \text { in } \mathcal{U}(B \otimes \hat{A} \otimes \hat{B}) .
$$


The character condition 2.9 for $\widehat{\mathrm{W}}^{B}$ gives:

$$
\widehat{\mathrm{W}}_{\theta 2}^{B}=\left(\bar{\beta} \otimes\left((\eta \otimes \hat{\pi}) \hat{\Delta}_{R}\right) \otimes \operatorname{id}_{\hat{B}}\right)\left(\widehat{\mathrm{W}}_{23}^{B} \widehat{\mathrm{W}}_{13}^{B}\right)=\chi_{\hat{\pi} 4} \cdot \widehat{\mathrm{W}}_{\eta 4}^{B} \widehat{\mathrm{W}}_{\bar{\beta} 4}^{B} \quad \text { in } \mathcal{U}(\mathbb{K}(\overline{\mathcal{L}} \otimes \mathcal{K} \otimes \mathcal{H}) \otimes \hat{B}) .
$$

Let us compute:

$$
\widehat{\mathrm{W}}_{\theta 3}^{B} \widehat{\mathrm{W}}_{1 \zeta}^{B}=\chi_{\hat{\pi} 5} \cdot \widehat{\mathrm{W}}_{\eta 5}^{B} \widehat{\mathrm{W}}_{\bar{\beta} 5}^{B} \widehat{\mathrm{W}}_{1 \hat{\eta}}^{B}=\chi_{\hat{\pi} 5} \cdot \widehat{\mathrm{W}}_{\eta 5}^{B} \widehat{\mathrm{W}}_{1 \hat{\eta}}^{B} \widehat{\mathrm{W}}_{\bar{\beta} 5}^{B}=\chi_{\hat{\pi} 5} \cdot \widehat{\mathrm{W}}_{1 \hat{\eta}}^{B} \widehat{\mathrm{W}}_{15}^{B} \widehat{\mathrm{W}}_{\eta 5}^{B} \widehat{\mathrm{W}}_{\bar{\beta} 5}^{B} .
$$

The first step uses 4.23), the second equality uses that $\widehat{\mathrm{W}}_{\bar{\beta} 5}^{B}$ and $\widehat{\mathrm{W}}_{1 \hat{\eta}}^{B}$ commute and the third equality uses that $(\eta, \hat{\eta})$ is an $\mathbb{H}$-Heisenberg pair. Hence 4.21 follows from 4.20 shifting leg numbers.

Similarly, 4.23) with the shifting of leg numbers gives 4.22):

$$
\widehat{\mathrm{W}}_{\theta 3}^{B} \widehat{\mathrm{W}}_{1 \xi}^{A}=\chi_{\hat{\pi} 5} \cdot \widehat{\mathrm{W}}_{\eta 5}^{B} \widehat{\mathrm{W}}_{\bar{\beta} 5}^{B} \widehat{\mathrm{W}}_{1 \hat{\pi}}^{A}=\chi_{\hat{\pi} 5} \cdot \widehat{\mathrm{W}}_{1 \hat{\pi}}^{A} \widehat{\mathrm{W}}_{\eta 5}^{B} \widehat{\mathrm{W}}_{\bar{\beta} 5}^{B}=\chi_{\xi 3} \cdot \widehat{\mathrm{W}}_{1 \xi}^{A} \cdot \chi_{\xi 3}^{*} \cdot \widehat{\mathrm{W}}_{\theta 3}^{B} .
$$

Finally, (5) and (6) follow from 4.20).

Notation 4.46. We write $\pi_{i}$ when the representation $\pi$ is acting on the $i$ th leg.

Proof of Proposition 4.44. Using 4.8] for $(\rho, \theta)$ involving $\widehat{\mathrm{W}}^{A}$ and $\widehat{\mathrm{W}}^{B}$ we obtain:

$$
\widehat{\mathrm{W}}_{\rho 2}^{A} \widehat{\mathrm{W}}_{\theta 3}^{B} \chi_{23}=\chi_{23} \widehat{\mathrm{W}}_{\theta 3}^{B} \widehat{\mathrm{W}}_{\rho 2}^{A} \quad \text { in } \mathcal{U}\left(\mathbb{K}\left(\mathcal{H}_{\mathcal{D}}\right) \otimes \hat{A} \otimes \hat{B}\right) .
$$

Equation 4.24 and (4.22) give:

$$
\widehat{\mathrm{W}}_{\rho_{1} \xi_{2}}^{A} \widehat{\mathrm{W}}_{\theta_{1} 3}^{B} \widehat{\mathrm{W}}_{\theta_{2} 3}^{B}=\chi_{\xi_{2} 3} \cdot \widehat{\mathrm{W}}_{\theta_{1} 3}^{B} \cdot \widehat{\mathrm{W}}_{\rho_{1} \xi_{2}}^{A} \cdot \chi_{\xi_{2} 3}^{*} \cdot \widehat{\mathrm{W}}_{\theta_{2} 3}^{B}=\chi_{\xi_{2} 3} \cdot \widehat{\mathrm{W}}_{\theta_{1} 3}^{B} \cdot \chi_{\xi_{2} 3}^{*} \cdot \widehat{\mathrm{W}}_{\theta_{2} 3}^{B} \cdot \widehat{\mathrm{W}}_{\rho_{1} \xi_{2}}^{A} \cdot
$$

The following computation takes place in $\mathcal{U}\left(\mathbb{K}\left(\mathcal{H}_{\mathcal{D}} \otimes \mathcal{H}_{\mathcal{D}}\right) \otimes \hat{B} \otimes \hat{A}\right)$ :

$$
\begin{aligned}
\widehat{\mathrm{W}}_{\theta_{2} 3}^{B} \widehat{\mathrm{W}}_{\rho_{2} 4}^{A} \widehat{\mathrm{W}}_{\theta_{1} \zeta_{2}}^{B} \widehat{\mathrm{W}}_{\rho_{1} \xi_{2}}^{A}\left(\widehat{\mathrm{W}}_{\rho_{2} 4}^{A}\right)^{*}\left(\widehat{\mathrm{W}}_{\theta_{2} 3}^{B}\right)^{*} & =\widehat{\mathrm{W}}_{\theta_{2} 3}^{B} \widehat{\mathrm{W}}_{\theta_{1} \zeta_{2}}^{B} \widehat{\mathrm{W}}_{\rho_{2} 4}^{A} \widehat{\mathrm{W}}_{\rho_{1} \xi_{2}}^{A}\left(\widehat{\mathrm{W}}^{A}\right)_{\rho_{2} 4}^{*}\left(\widehat{\mathrm{W}}^{B}\right)_{\theta_{2} 3}^{*} \\
& =\widehat{\mathrm{W}}_{\theta_{1} \zeta_{2}}^{B} \cdot \chi_{\xi_{2} 3} \cdot \widehat{\mathrm{W}}_{\theta_{\theta_{3} 3}}^{B} \cdot \chi_{\xi_{2} 3}^{*} \cdot \widehat{\mathrm{W}}_{\theta_{\theta_{3}}}^{B} \widehat{\mathrm{W}}_{\rho_{1} \xi_{2}}^{A} \widehat{\mathrm{W}}_{\rho_{1} 4}^{A}\left(\widehat{\mathrm{W}}^{B}\right)_{\theta_{2} 3}^{*} \\
& =\widehat{\mathrm{W}}_{\rho_{1} \zeta_{2}}^{B} \widehat{\mathrm{W}}_{\rho_{1} \xi_{2}}^{A} \widehat{\mathrm{W}}_{\theta_{13} 3}^{B} \widehat{\mathrm{W}}_{\theta_{2} 3}^{B}\left(\widehat{\mathrm{W}}^{B}\right)_{\theta_{2} 3}^{*} \widehat{\mathrm{W}}_{\rho_{1} 4}^{A} \\
& =\widehat{\mathrm{W}}_{\theta_{1} \zeta_{2}}^{B} \widehat{\mathrm{W}}_{\rho_{1} \xi_{2}}^{A} \widehat{\mathrm{W}}_{\theta_{1} 3}^{B} \widehat{\mathrm{W}}_{\rho_{1} 4}^{A} .
\end{aligned}
$$

The first equality uses the commutation between $\rho$ and $\zeta$, the second equality uses 4.21 and statement 11 in Lemma 4.45 , the third equality uses 4.25 and that $\widehat{\mathrm{W}}_{\theta_{2} 3}^{B}, \widehat{\mathrm{W}}_{\rho_{14}}^{A}$ commute, the last equality is trivial.

Applying $\xi$ and $\zeta$ on the third and fourth leg on both sides of the last expression gives the pentagon equation (2.4) for $\mathbb{W}^{\mathcal{D}} \in \mathcal{U}\left(\mathcal{H}_{\mathcal{D}} \otimes \mathcal{H}_{\mathcal{D}}\right)$.

Since $\widehat{\mathrm{W}}_{\theta 2}^{B} \in \mathcal{U}\left(\mathbb{K}\left(\mathcal{H}_{\mathcal{D}}\right) \otimes \hat{B}\right)$ is a corepresentation of $\widehat{\mathbb{H}}$ on $\mathcal{H}_{\mathcal{D}}$, by 49 . Theorem 1.7], it is adapted

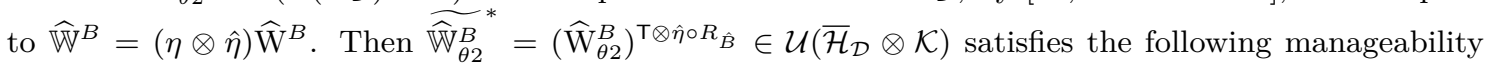
condition:

$$
\left(x \otimes k\left|\widehat{\mathrm{W}}_{\theta \hat{\eta}}^{B}\right| z \otimes k^{\prime}\right)=\left(\bar{z} \otimes Q_{B}(k)\left|\widetilde{\widehat{\mathbb{W}}_{\theta 2}^{B}}\right| \bar{x} \otimes Q_{B}^{-1}\left(k^{\prime}\right)\right)
$$

for all $x, z \in \mathcal{H}_{\mathcal{D}}, k \in \mathcal{D} \operatorname{Dom}\left(Q_{B}\right)$ and $k^{\prime} \in \mathcal{D}$ om $\left(Q_{B}^{-1}\right)$.

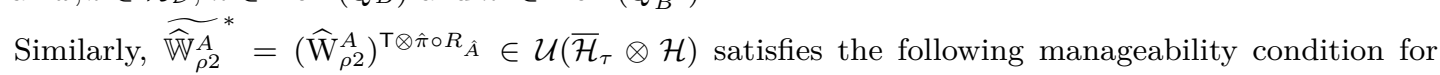
$\widehat{\mathrm{W}}_{\rho 2}^{A} \in \mathcal{U}\left(\mathbb{K}\left(\mathcal{H}_{\mathcal{D}}\right) \otimes \hat{A}\right):$

$$
\left(x \otimes h\left|\widehat{\mathrm{W}}_{\rho \tilde{\pi}}^{A}\right| z \otimes h^{\prime}\right)=\left(\bar{z} \otimes Q_{A}(h)\left|\widetilde{\mathbb{\mathbb { W }}_{\rho 2}^{A}}\right| \bar{x} \otimes Q_{A}^{-1}\left(h^{\prime}\right)\right)
$$

for all $x, z \in \mathcal{H}_{\mathcal{D}}, h \in \mathcal{D} \operatorname{om}\left(Q_{A}\right)$ and $h^{\prime} \in \mathcal{D}$ om $\left(Q_{A}^{-1}\right)$. 
Let $\left\{e_{i}\right\}_{i=1,2, \cdots}$ be an orthonormal basis of $\mathcal{H}_{\mathcal{D}}$. Then we get Definition 2.10 (ii) for $\mathbb{W}^{\mathcal{D}}$ as follows:

$$
\begin{aligned}
& \left(x \otimes k \otimes h\left|\widehat{\mathrm{W}}_{\theta \hat{\eta}_{2}}^{B} \widehat{\mathrm{W}}_{\rho \hat{\pi}_{3}}^{A}\right| z \otimes k^{\prime} \otimes h^{\prime}\right) \\
& =\left(x \otimes k \otimes h\left|\widehat{\mathrm{W}}_{\theta \hat{\eta}_{2}}^{B} \sum_{i}\left(\mid e_{i}\right)\left(e_{i} \mid \otimes 1_{\hat{B}} \otimes 1_{\hat{A}}\right) \widehat{\mathrm{W}}_{\rho \hat{\pi}_{3}}^{A}\right| z \otimes k^{\prime} \otimes h^{\prime}\right) \\
& =\sum_{i}\left(x \otimes k\left|\widehat{\mathrm{W}}_{\theta \hat{\eta}_{2}}^{B}\right| e_{i} \otimes k^{\prime}\right) \cdot\left(e_{i} \otimes h\left|\widehat{\mathrm{W}}_{\rho \hat{\pi}_{3}}^{A}\right| z \otimes h^{\prime}\right) \\
& =\sum_{i}\left(\bar{z} \otimes Q_{A}(h)\left|\widetilde{\widehat{\mathbb{W}}_{\rho 3}^{A}}\right| \overline{e_{i}} \otimes Q_{A}^{-1}\left(h^{\prime}\right)\right) \cdot\left(\overline{e_{i}} \otimes Q_{B}(k)\left|\widetilde{\widehat{\mathbb{W}}_{\theta 2}^{B}}\right| \bar{x} \otimes Q_{B}^{-1}\left(k^{\prime}\right)\right) \\
& =\left(\bar{z} \otimes Q_{B}(k) \otimes Q_{A}(h)\left|\widetilde{\widehat{\mathbb{W}}_{\rho 3}^{A}} \widetilde{\widehat{\mathbb{W}}_{\theta 2}^{B}}\right| \bar{x} \otimes Q_{B}^{-1}\left(k^{\prime}\right) \otimes Q_{A}^{-1}\left(h^{\prime}\right)\right) .
\end{aligned}
$$

Recall the definition of $(\alpha, \beta)$ from Lemma 4.11 and identify $\mathcal{H}_{\mathcal{D}}=\overline{\mathcal{H}} \otimes \overline{\mathcal{K}} \otimes \mathcal{K} \otimes \mathcal{H}$.

Equation 4.23 gives $\widehat{\mathrm{W}}_{\theta \zeta}^{B}=\chi_{47} \widehat{\mathbb{W}}_{37}^{B}\left(\widehat{\mathrm{W}}^{B}\right)_{27}^{1 \otimes \hat{\eta} \circ R_{\hat{B}}} \in \mathcal{U}\left(\mathcal{H}_{\mathcal{D}} \otimes \mathcal{H}_{\mathcal{D}}\right)$. Similarly, we show $\widehat{\mathrm{W}}_{\rho \xi}^{A}=$

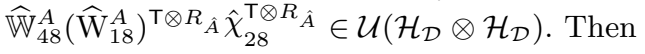

$$
\mathbb{W}^{\mathcal{D}}=\chi_{47} \widehat{\mathbb{W}}_{37}^{B}{\widetilde{\mathbb{W}^{B}}}_{27}^{*} \widehat{\mathbb{W}}_{48}^{A} \widetilde{\mathbb{W}}^{A}{ }_{18}^{*} \widetilde{\widehat{\chi}}_{28}^{*} \quad \text { in } \mathcal{U}(\overline{\mathcal{H}} \otimes \overline{\mathcal{K}} \otimes \mathcal{K} \otimes \mathcal{H} \otimes \overline{\mathcal{H}} \otimes \overline{\mathcal{K}} \otimes \mathcal{K} \otimes \mathcal{H}) .
$$

Define $\hat{Q}_{\tau}:=\left(Q_{A}^{-1}\right)^{\top} \otimes\left(Q_{B}^{-1}\right)^{\top} \otimes Q_{B} \otimes Q_{A}$ and $Q_{\tau}:=1_{\overline{\mathcal{H}}} \otimes 1_{\overline{\mathcal{K}}} \otimes Q_{B} \otimes Q_{A}$. Clearly, $\hat{Q}_{\tau}$ and $Q_{\tau}$ are positive, self-adjoint operators acting on $\mathcal{H}_{\mathcal{D}}$ with trivial kernel.

The commutation relations Definition 2.10(i) Theorem 2.13|(6)|(ii) and Lemma 3.13 (3.12) and (3.13) yield $\mathbb{W}^{\mathcal{D}}\left(\hat{Q}_{\tau} \otimes Q_{\tau}\right)\left(\mathbb{W}^{\mathcal{D}}\right)^{*}=\hat{Q}_{\tau} \otimes Q_{\tau}$.

\subsubsection{Generalised Drinfeld doubles}

We first describe the quantum group associated to the dual of the multiplicative unitary $\mathbb{W}^{\mathcal{D}} \in \mathcal{U}\left(\mathcal{H}_{\mathcal{D}} \otimes \mathcal{H}_{\mathcal{D}}\right)$. The definition of the faithful representations $\xi$ and $\zeta$ in 4.20 allows us to view $\mathbb{W}^{\mathcal{D}}$ as a unitary multiplier of $\mathbb{K}\left(\mathcal{H}_{\mathcal{D}}\right) \otimes \hat{B} \otimes \hat{A}$. According to the general theory of modular multiplicative unitaries [38], the dual of $\mathbb{W}^{\mathcal{D}}$ is again modular. This gives rise to a $\mathrm{C}^{*}$-algebra $\mathcal{D}^{\chi}$ defined by:

$$
\mathcal{D}^{\chi}:=\left\{\left(\operatorname{id}_{\mathcal{H}_{\mathcal{D}}} \otimes \omega\right) \mathbb{W}^{\mathcal{D}}: \omega \in(\hat{B} \otimes \hat{A})^{\prime}\right\}^{\mathrm{CLS}} \subset \mathbb{B}\left(\mathcal{H}_{\mathcal{D}}\right),
$$

where CLS abbreviates closed linear spans.

The set of continuous linear functionals of the form $\eta \otimes \psi$ for $\eta \in \hat{B}^{\prime}, \psi \in \hat{A}^{\prime}$ is linearly weak ${ }^{*}$ dense in $(\hat{B} \otimes \hat{A})^{\prime}$. Thus $\mathcal{D}^{\chi}=\theta(B) \cdot \rho(A)$ and $\mathbb{W}^{\mathcal{D}} \in \mathcal{U}\left(\mathcal{D}^{\chi} \otimes \hat{B} \otimes \hat{A}\right)$. The embeddings $\rho$ of $A$ and $\theta$ of $B$ into $\mathcal{D}^{\chi}$ satisfy the $\chi$-Drinfeld commutation relation.

Next we establish the well-definedness of the comultiplication $\Delta_{\mathcal{D}^{\chi}} \in \operatorname{Mor}\left(\mathcal{D}^{\chi}, \mathcal{D}^{\chi} \otimes \mathcal{D}^{\chi}\right)$ on $\mathcal{D}^{\chi}$ by $\Delta_{\mathcal{D}^{\chi}}(\theta(b) \cdot \rho(a)):=\left((\theta \otimes \theta) \Delta_{B}(b) \cdot(\rho \otimes \rho) \Delta_{A}(a)\right)$ for $a \in \hat{A}, b \in \hat{B}$.

Definition 4.47. The quantum group $\mathfrak{D}^{\chi}(\mathbb{G}, \widehat{\mathbb{H}}):=\left(\mathcal{D}^{\chi}, \Delta_{\mathcal{D}^{\chi}}\right)$ is called the $\chi$-Drinfeld double.

We must show that $\Delta_{\mathcal{D}^{x}}$ is implemented by the dual of $\mathbb{W}^{\mathcal{D}}$. The character condition 2.9 for $\widehat{\mathrm{W}}^{A}$ and $\widehat{\mathrm{W}}^{B}$ gives:

$$
\begin{aligned}
\left(\Delta_{\mathcal{D}^{\chi}} \otimes \operatorname{id}_{\hat{B} \otimes \hat{A}}\right) \widehat{\mathrm{W}}_{\rho 2}^{B} \widehat{\mathrm{W}}_{\theta 3}^{A} & =\left(\left((\theta \otimes \theta) \Delta_{B} \otimes \operatorname{id}_{\hat{B}}\right) \widehat{\mathrm{W}}^{B}\right)_{123}\left(\left((\rho \otimes \rho) \Delta_{A} \otimes \mathrm{id}_{\hat{A}}\right) \widehat{\mathrm{W}}^{A}\right)_{124} \\
& =\widehat{\mathrm{W}}_{\theta_{2} 3}^{B} \widehat{\mathrm{W}}_{\rho_{2} 4}^{A} \widehat{\mathrm{W}}_{\theta_{1} 3}^{B} \widehat{\mathrm{W}}_{\rho_{14} 4}^{A} .
\end{aligned}
$$

Equivalently, $\left(\Delta_{\mathcal{D}^{\chi}} \otimes \operatorname{id}_{\hat{B} \otimes \hat{A}}\right) \mathbb{W}^{\mathcal{D}}=\mathbb{W}_{234}^{\mathcal{D}} \mathbb{W}_{134}^{\mathcal{D}}$ in $\mathcal{U}\left(\mathcal{D}^{\chi} \otimes \mathcal{D}^{\chi} \otimes \hat{B} \otimes \hat{A}\right)$. Slicing the last two legs of both sides of the last expression by $\omega \in(\hat{B} \otimes \hat{A})^{\prime}$ and then using the pentagon equation for $\mathbb{W}^{\mathcal{D}}$ gives:

$$
\Delta_{\mathcal{D}^{\chi}}(\theta(b) \cdot \rho(a))=\left(\widehat{\mathbb{W}}^{\mathcal{D}}\right)\left(\theta(b) \rho(a) \otimes 1_{\mathcal{D} \chi}\right)\left(\widehat{\mathbb{W}}^{\mathcal{D}}\right)^{*},
$$

for $a \in A$ and $b \in B$. Thus $\Delta_{\mathcal{D}^{x}}$ is implemented by the dual multiplicative unitary $\widehat{\mathbb{W}}^{\mathcal{D}} \in \mathcal{U}\left(\mathcal{H}_{\mathcal{D}} \otimes \mathcal{H}_{\mathcal{D}}\right)$.

Finally, $\rho \in \operatorname{Mor}\left(\hat{A}, \mathcal{D}^{\chi}\right)$ and $\theta \in \operatorname{Mor}\left(\hat{B}, \mathcal{D}^{\chi}\right)$ are Hopf ${ }^{*}$-homomorphisms. Therefore, $\widehat{\mathrm{W}}_{\rho 2}^{A} \in \mathcal{U}\left(\mathcal{D}^{\chi} \otimes \hat{A}\right)$ and $\widehat{\mathrm{W}}_{\theta 2}^{B} \in \mathcal{U}\left(\mathcal{D}^{\chi} \otimes \hat{B}\right)$ are bicharacters. 


\subsubsection{Generalised quantum codoubles}

If $\widehat{\mathbb{H}}=\mathbb{G}$, the quantum codouble was described in 34 as dual to the Drinfeld double. In this section, we construct the generalised quantum codouble in the same spirit.

Define $\sigma^{\chi}: \hat{B} \otimes \hat{A} \rightarrow \hat{A} \otimes \hat{B}$, by $\sigma^{\chi}(\hat{b} \otimes \hat{a}):=\chi(\hat{a} \otimes \hat{b}) \chi^{*}$ for $\hat{a} \in \hat{A}, \hat{b} \in \hat{B}$. This is called flip twisted by $\chi$. Next we define $\widehat{\mathcal{D}}^{\chi}:=\hat{B} \otimes \hat{A}$ and $\Delta_{\widehat{\mathcal{D}}^{\chi}} \in \operatorname{Mor}\left(\widehat{\mathcal{D}}^{\chi}, \widehat{\mathcal{D}}^{\chi} \otimes \widehat{\mathcal{D}}^{\chi}\right)$ by

$$
\Delta_{\widehat{\mathcal{D}}^{x}}:=\sigma_{23}^{\chi} \circ\left(\hat{\Delta}_{B} \otimes \hat{\Delta}_{A}\right) .
$$

Definition 4.48. The pair $\mathfrak{D}^{\chi}(\mathbb{G}, \widehat{\mathbb{H}})^{\wedge}:=\left(\widehat{\mathcal{D}}^{\chi}, \Delta_{\widehat{\mathcal{D}}^{\chi}}\right)$ is called $\chi$-quantum codouble.

Proposition 4.49. The comultiplication $\Delta_{\widehat{\mathcal{D}}^{\chi}}$ is implemented by $\mathbb{W}^{\mathcal{D}} \in \mathcal{U}\left(\mathcal{D}^{\chi} \otimes \hat{B} \otimes \hat{A}\right)$. Moreover, $\left(\widehat{\mathcal{D}}^{\chi}, \Delta_{\widehat{\mathcal{D}}^{\chi}}\right)$ is a bisimplifiable Hopf-( $\mathrm{C}^{*}$-algebra.

Proof. Realise $\mathbb{W}^{\mathcal{D}}$ as a unitary element in the multiplier algebra $\mathbb{K}\left(\mathcal{H}_{\mathcal{D}}\right) \otimes \hat{B} \otimes \hat{A}$. Then we compute:

$$
\begin{aligned}
\widehat{\mathrm{W}}_{\theta 4}^{B} \widehat{\mathrm{W}}_{\rho 5}^{A} \widehat{\mathrm{W}}_{1 \xi}^{A} \widehat{\mathrm{W}}_{2 \zeta}^{B}\left(\widehat{\mathrm{W}}_{\rho 5}^{A}\right)^{*}\left(\widehat{\mathrm{W}}_{\theta 4}^{B}\right)^{*} & =\widehat{\mathrm{W}}_{\theta 4}^{B} \widehat{\mathrm{W}}_{1 \xi}^{A} \widehat{\mathrm{W}}_{15}^{A} \widehat{\mathrm{W}}_{\rho 5}^{A} \widehat{\mathrm{W}}_{2 \zeta}^{B}\left(\widehat{\mathrm{W}}_{\rho 5}^{A}\right)^{*}\left(\widehat{\mathrm{W}}_{\theta 4}^{B}\right)^{*} \\
& =\widehat{\mathrm{W}}_{\theta 4}^{B} \widehat{\mathrm{W}}_{1 \xi}^{A} \widehat{\mathrm{W}}_{15}^{A} \widehat{\mathrm{W}}_{2 \zeta}^{B}\left(\widehat{\mathrm{W}}_{\theta 4}^{B}\right)^{*} \\
& =\widehat{\mathrm{W}}_{\theta 4}^{B} \widehat{\mathrm{W}}_{1 \xi}^{A} \widehat{\mathrm{W}}_{15}^{A}\left(\widehat{\mathrm{W}}_{\theta 4}^{B}\right)^{*} \cdot \chi_{\xi 4} \cdot \widehat{\mathrm{W}}_{2 \zeta}^{B} \widehat{\mathrm{W}}_{24}^{B} \cdot \chi_{\xi 4}^{*} \\
& =\widehat{\mathrm{W}}_{\theta 4}^{B} \widehat{\mathrm{W}}_{1 \xi}^{A}\left(\widehat{\mathrm{W}}_{\theta 4}^{B}\right)^{*} \widehat{\mathrm{W}}_{15}^{A} \cdot \chi_{\xi 4} \cdot \widehat{\mathrm{W}}_{2 \zeta}^{B} \widehat{\mathrm{W}}_{24}^{B} \cdot \chi_{\xi 4}^{*} \\
& =\chi_{\xi 4} \cdot \widehat{\mathrm{W}}_{1 \xi}^{A} \cdot \chi_{\xi 4}^{*} \cdot \widehat{\mathrm{W}}_{15}^{A} \cdot \chi_{\xi 4} \cdot \widehat{\mathrm{W}}_{2 \zeta}^{B} \widehat{\mathrm{W}}_{24}^{B} \cdot \chi_{\xi 4}^{*} \\
& =\chi_{\xi 4} \cdot \widehat{\mathrm{W}}_{1 \xi}^{A} \widehat{\mathrm{W}}_{15}^{A} \widehat{\mathrm{W}}_{2 \zeta}^{B} \widehat{\mathrm{W}}_{24}^{B} \cdot \chi_{\xi 4}^{*} .
\end{aligned}
$$

The first equality uses Lemma 4.45 1, the second equality uses that $\rho$ and $\zeta$ commute, the third equality uses 4.21 and that $\xi$ and $\zeta$ commute, the fourth equality follows because $\widehat{W}_{15}^{A}$ and $\widehat{\mathrm{W}}_{\theta 4}^{B}$ commute, the fifth equality uses 4.22 , and the last equality follows because $\widehat{\mathrm{W}}_{15}^{A}$ and $\chi_{\xi 4}$ commute.

Taking slices on the first two legs by $\omega \otimes \omega^{\prime}$ for $\omega \in A^{\prime}, \omega^{\prime} \in B^{\prime}$ on both sides of the last expression gives

$$
\left(\mathbb{W}^{\mathcal{D}}\right)\left(\xi(\hat{a}) \zeta(\hat{b}) \otimes 1_{\hat{B} \otimes \hat{A}}\right)\left(\mathbb{W}^{\mathcal{D}}\right)^{*}=\chi_{\xi 4}\left(\hat{\Delta}_{B}(\hat{b})_{\zeta 2} \hat{\Delta}_{A}(\hat{a})_{\xi 3}\right) \chi_{\xi 4}^{*} .
$$

Since the representations $\xi$ and $\zeta$ are faithful, we conclude that $\hat{\Delta}_{\widehat{\mathcal{D}}^{x}}$ is implemented by $\mathbb{W}^{\mathcal{D}}$. The pentagon equation for $\mathbb{W}^{\mathcal{D}}$ shows that $\Delta_{\widehat{\mathcal{D}}^{x}}$ is coassociative.

We know that $\hat{\Delta}_{A}(A) \cdot\left(1_{\hat{A}} \otimes \hat{A}\right)=\hat{A} \otimes \hat{A}$. Therefore,

$$
\begin{aligned}
\Delta_{\widehat{\mathcal{D}}^{\chi}}\left(\widehat{\mathcal{D}}^{\chi}\right) \cdot\left(1_{\widehat{\mathcal{D}}^{\chi}} \otimes \widehat{\mathcal{D}}^{\chi}\right) & =\chi_{23}\left(\hat{\Delta}_{B}(\hat{B})_{13} \hat{\Delta}_{A}(\hat{A})_{24}\right) \chi_{23}^{*} \cdot\left(1_{\hat{B} \otimes \hat{A}} \otimes \hat{B} \otimes \hat{A}\right) \\
& =\chi_{23}\left(\hat{\Delta}_{B}(\hat{B})_{13}\left(\hat{\Delta}_{A}(\hat{A}) \cdot\left(1_{\hat{A}} \otimes \hat{A}\right)\right)_{24}\right) \chi_{23}^{*}\left(1_{\hat{B} \otimes \hat{A}} \otimes \hat{B} \otimes 1_{\hat{A}}\right) \\
& =\chi_{23}\left(\hat{\Delta}_{B}(\hat{B})_{13}(\hat{A} \otimes \hat{A})_{24}\right) \chi_{23}^{*}\left(1_{\hat{B} \otimes \hat{A}} \otimes \hat{B} \otimes 1_{\hat{A}}\right) .
\end{aligned}
$$

Now the character condition on the second leg of $\chi$ is equivalent to

$$
\widehat{\mathrm{W}}_{\eta^{\prime} 3}^{B} \chi_{1 \hat{\eta}^{\prime}}=\chi_{1 \hat{\eta}^{\prime}} \chi_{13} \widehat{\mathrm{W}}_{\eta^{\prime} 3}^{B} \quad \text { in } \mathcal{U}\left(\hat{A} \otimes \mathbb{K}\left(\mathcal{H}_{\eta^{\prime}}\right) \otimes \hat{B}\right),
$$

where $\left(\eta, \hat{\eta}^{\prime}\right)$ is an $\mathbb{H}$-Heisenberg pair acting on $\mathcal{H}_{\eta^{\prime}}$. We also know $\hat{\Delta}_{B}(B) \cdot\left(1_{\hat{B}} \otimes \hat{B}\right)=\hat{B} \otimes \hat{B}$ and $\left(\hat{\eta}^{\prime}(\hat{B}) \otimes \hat{A}\right) \hat{\chi}_{\hat{\eta}^{\prime} 2}=\hat{\eta}^{\prime}(\hat{B}) \otimes \hat{A}$. Hence

$$
\Delta_{\widehat{\mathcal{D}}^{\chi}}(\hat{B} \otimes \hat{A})_{\hat{\eta}^{\prime} 234} \cdot\left(1_{\mathcal{H}_{\eta^{\prime}}} \otimes 1_{\hat{A}} \otimes \hat{B} \otimes \hat{A}\right)=\chi_{23} \widehat{\mathrm{W}}_{\eta^{\prime} 3}^{B}\left(\hat{\eta}^{\prime}(\hat{B}) \otimes \hat{A} \otimes 1_{\hat{B}} \otimes \hat{A}\right)\left(\chi_{23} \widehat{\mathrm{W}}_{\eta^{\prime} 3}^{B}\right)^{*}\left(1_{\mathcal{H}_{\eta^{\prime}}} \otimes 1_{\hat{A}} \otimes \hat{B} \otimes 1_{\hat{A}}\right) .
$$

Rewriting 4.4 for $\chi \in \mathcal{U}(\hat{A} \otimes \hat{B})$ we get

$$
\hat{\chi}_{\hat{\eta}^{\prime} 2} \widehat{\mathrm{W}}_{\eta^{\prime} 3}^{B}=\chi_{23} \widehat{\mathrm{W}}_{\eta^{\prime} 3}^{B} \hat{\chi}_{\hat{\eta}^{\prime} 2} \quad \text { in } \mathcal{U}\left(\mathbb{K}\left(\mathcal{H}_{\eta^{\prime}}\right) \otimes \hat{A} \otimes \hat{B}\right) .
$$


Therefore, using 4.30, we obtain

$$
\begin{aligned}
& \Delta_{\widehat{\mathcal{D}}^{\chi}}(\hat{B} \otimes \hat{A})_{\hat{\eta}^{\prime} 234} \cdot\left(1_{\mathcal{H}_{\eta^{\prime}}} \otimes 1_{\hat{A}} \otimes \hat{B} \otimes \hat{A}\right) \\
& =\chi_{23} \widehat{\mathrm{W}}_{\eta 3}^{B}\left(\hat{\eta}(\hat{B}) \otimes \hat{A} \otimes 1_{\hat{B}} \otimes \hat{A}\right) \hat{\chi}_{\hat{\eta} 2}\left(\widehat{\mathrm{W}}_{\eta 3}^{B}\right)^{*} \hat{\chi}_{\hat{\eta} 2}^{*}\left(1_{\mathcal{H}_{\eta}} \otimes 1_{\hat{A}} \otimes \hat{B} \otimes 1_{\hat{A}}\right) \\
& =\chi_{23} \widehat{\mathrm{W}}_{\eta 3}^{B}\left(\hat{\eta}(\hat{B}) \otimes \hat{A} \otimes 1_{\hat{B}} \otimes \hat{A}\right)\left(1_{\mathcal{H}_{\eta}} \otimes 1_{\hat{A}} \otimes \hat{B} \otimes 1_{\hat{A}}\right)\left(\widehat{\mathrm{W}}_{\eta 3}^{B}\right)^{*} \hat{\chi}_{\hat{\eta} 2}^{*} \\
& =\chi_{23} \widehat{\mathrm{W}}_{\eta 3}^{B}(\hat{\eta}(\hat{B}) \otimes \hat{A} \otimes \hat{B} \otimes \hat{A})\left(\widehat{\mathrm{W}}_{\eta 3}^{B}\right)^{*} \hat{\chi}_{\hat{\eta}^{2}}^{*}=\hat{\eta}(\hat{B}) \otimes \hat{A} \otimes \hat{B} \otimes \hat{A} .
\end{aligned}
$$

Since $\hat{\eta}$ is faithful, we get $\Delta_{\widehat{\mathcal{D}}^{\chi}}\left(\widehat{\mathcal{D}}^{\chi}\right) \cdot\left(1_{\widehat{\mathcal{D}}^{\chi}} \otimes \widehat{\mathcal{D}}^{\chi}\right)=\widehat{\mathcal{D}}^{\chi} \otimes \widehat{\mathcal{D}}^{\chi}$. A similar computation yields $\Delta_{\widehat{\mathcal{D}}^{\chi}}\left(\widehat{\mathcal{D}}^{\chi}\right)$. $\left(\widehat{\mathcal{D}}^{\chi} \otimes 1_{\widehat{\mathcal{D}}^{\chi}}\right)=\widehat{\mathcal{D}}^{\chi} \otimes \widehat{\mathcal{D}}^{\chi} ;$ hence $\left(\widehat{\mathcal{D}}^{\chi}, \Delta_{\widehat{\mathcal{D}}^{\chi}}\right)$ is bisimplifiable.

The proposition below shows that $\mathfrak{D}^{\chi}(\mathbb{G}, \widehat{\mathbb{H}})^{\wedge}$ is generated by $\mathbb{W}^{\mathcal{D}} \in \mathcal{U}\left(\mathcal{H}_{\mathcal{D}} \otimes \mathcal{H}_{\mathcal{D}}\right)$.

Proposition 4.50. The space of slices $\left\{\left(\omega \otimes \operatorname{id}_{\mathcal{H}_{\mathcal{D}}}\right) \mathbb{W}^{\mathcal{D}}: \omega \in \mathbb{B}\left(\mathcal{H}_{\mathcal{D}}\right)_{*}\right\}$ is linearly dense in $1_{\overline{\mathcal{H}} \otimes \overline{\mathcal{K}}} \otimes \widehat{\mathcal{D}}^{\chi}$.

Proof. By Proposition 3.10 and manageability of $\chi$, discussed in Section 3.1.3 we can write $\tilde{\chi}^{*}=\chi^{\mathrm{R}_{\hat{A}}}{ }^{\mathrm{T} \otimes \mathrm{id}_{\mathcal{K}}}$ and $\widetilde{\widehat{\chi}}^{*}=\hat{\chi}^{\mathrm{R}} \hat{B}^{\mathrm{T} \otimes \mathrm{id} \mathcal{H}}$.

Throughout the proof we identify $A, \hat{A}, B, \hat{B}$ with their images under the faithful representations $\pi, \hat{\pi}$, $\eta, \hat{\eta}$ to avoid notational complications.

Then we rewrite 4.28 as

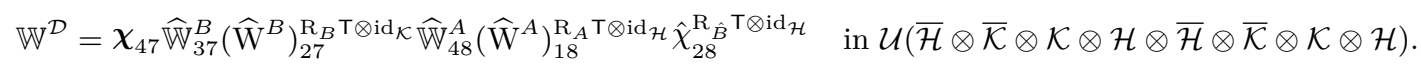

Let $L=\left\{\left(\omega \otimes \operatorname{id}_{\mathcal{H}_{\mathcal{D}}}\right) \mathbb{W}^{\mathcal{D}}: \omega \in \mathbb{B}\left(\mathcal{H}_{\mathcal{D}}\right)_{*}\right\}$. Using the leg numbering notation for functionals and replacing $\omega \in$ $\mathbb{B}\left(\mathcal{H}_{\mathcal{D}}\right)_{*}$ by $\mu \otimes \epsilon \otimes \nu \otimes v$ we have

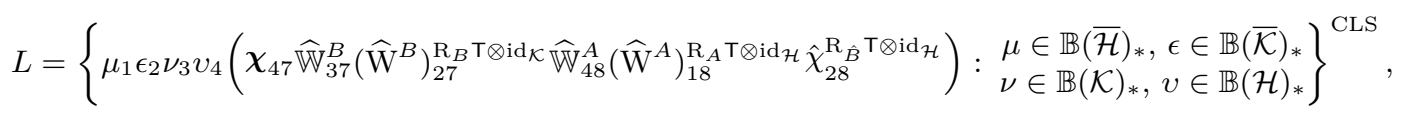

where CLS abbreviates closed linear spans. Replacing $\left(\nu \otimes \operatorname{id}_{\mathcal{K}}\right) \widehat{\mathbb{W}}^{B}$ by $\hat{b} \in \hat{B}$ we get:

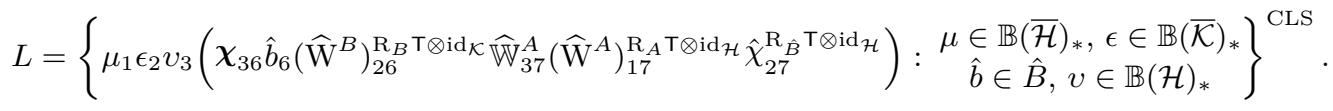

Replacing $\epsilon \in \mathbb{B}(\overline{\mathcal{H}})_{*}$ by $b^{\mathrm{R}_{B} \mathrm{~T}} \cdot \epsilon$, where $b \in B$, this becomes:

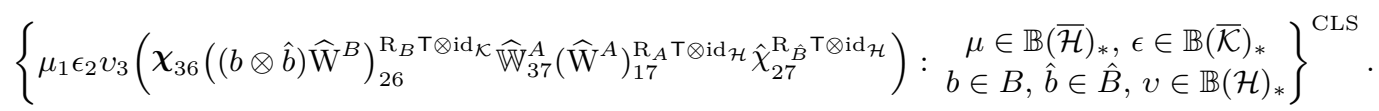

Since $\widehat{\mathrm{W}}^{B} \in \mathcal{U}(B \otimes \hat{B})$, we replace $(B \otimes \hat{B}) \widehat{\mathrm{W}}^{B}$ by $B \otimes \hat{B}$ :

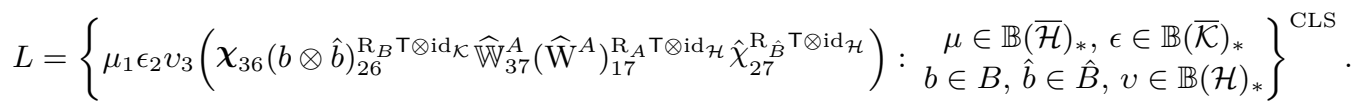

Replacing $b^{\mathrm{R}_{B} \mathrm{~T}} \cdot \epsilon$ by $\epsilon$, where $b \in B$ and $\epsilon \in \mathbb{B}(\overline{\mathcal{H}})_{*}$, we get:

$$
L=\left\{\mu_{1} \epsilon_{2} v_{3}\left(\chi_{36} \hat{b}_{6} \widehat{\mathbb{W}}_{37}^{A}\left(\widehat{\mathrm{W}}^{A}\right)_{17}^{\mathrm{R}_{A} \mathrm{~T} \otimes \operatorname{id} \mathcal{H}} \hat{\chi}_{27}^{\mathrm{R}_{\hat{B}}{ }^{\mathrm{T}} \otimes \mathrm{id}_{\mathcal{H}}}\right): \begin{array}{c}
\mu \in \mathbb{B}(\overline{\mathcal{H}})_{*}, \epsilon \in \mathbb{B}(\overline{\mathcal{K}})_{*} \\
\hat{b} \in \hat{B}, v \in \mathbb{B}(\mathcal{H})_{*}
\end{array}\right\}^{\mathrm{CLS}} .
$$

Next we replace $\left(\mu \otimes \operatorname{id}_{\mathcal{H}}\right)\left(\widehat{\mathrm{W}}^{A}\right)^{\mathrm{R}_{A} \mathrm{~T} \otimes \operatorname{id}_{\mathcal{H}}}$ by $\hat{a} \in \hat{A}$, and $\epsilon \in \mathbb{B}(\overline{\mathcal{H}})_{*}$ by $\hat{b}^{\prime \mathrm{R}_{\hat{B}}} \cdot \epsilon$, where $\hat{b}^{\prime} \in \hat{B}$; we get

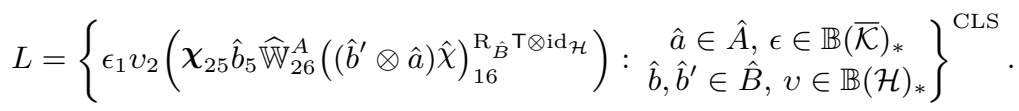


Since $\chi \in \mathcal{U}(\hat{A} \otimes \hat{B})$, we may replace $(\hat{B} \otimes \hat{A}) \hat{\chi}$ by $\hat{B} \otimes \hat{A}$ and $(\hat{B} \otimes \hat{A})^{\mathrm{R}_{\hat{B}}} \hat{\mathrm{T}}^{\mathrm{id}} \mathcal{H}$ by $\hat{B}^{\mathrm{T}} \otimes \hat{A}$ :

$$
L=\left\{\epsilon_{1} v_{2}\left(\chi_{25} \hat{b}_{5} \widehat{\mathbb{W}}_{26}^{A} \hat{b}_{1}^{\prime \top} \hat{a}_{6}\right): \begin{array}{c}
\hat{a} \in \hat{A}, \epsilon \in \mathbb{B}(\overline{\mathcal{K}})_{*} \\
\hat{b}, \hat{b}^{\prime} \in \hat{B}, v \in \mathbb{B}(\mathcal{H})_{*}
\end{array}\right\}^{\mathrm{CLS}}=\left\{v_{1}\left(\chi_{14} \hat{b}_{4} \widehat{\mathbb{W}}_{15}^{A} \hat{a}_{5}\right): \begin{array}{c}
\hat{a} \in \hat{A}, \\
\hat{b} \in \hat{B}, v \in \mathbb{B}(\mathcal{H})_{*}
\end{array}\right\}^{\mathrm{CLS}} .
$$

Replacing $v \in \mathbb{B}(\mathcal{H})_{*}$ by $v \cdot a$, where $a \in A$, we get:

$$
L=\left\{v_{1}\left(\chi_{14} \hat{b}_{4}\left(\widehat{\mathbb{W}}^{A}(a \otimes \hat{a})\right)_{15}\right): \begin{array}{c}
\hat{a} \in \hat{A}, a \in A \\
\hat{b} \in \hat{B}, v \in \mathbb{B}(\mathcal{H})_{*}
\end{array}\right\}^{\mathrm{CLS}} .
$$

Since $\widehat{\mathbb{W}}^{A} \in \mathcal{U}(A \otimes \hat{A})$ we may replace $\widehat{\mathbb{W}}^{A}(A \otimes \hat{A})$ by $A \otimes \hat{A}$ :

$$
L=\left\{v_{1}\left(\chi_{14} \hat{b}_{4} a_{1} \hat{a}_{5}\right): \begin{array}{c}
\hat{a} \in \hat{A}, a \in A \\
\hat{b} \in \hat{B}, v \in \mathbb{B}(\mathcal{H})_{*}
\end{array}\right\}^{\mathrm{CLS}} .
$$

Next we replace $v_{1} \cdot a$ by $v$ and then replace $v$ by $v \cdot \hat{a}^{\prime}$, where $\hat{a}^{\prime} \in \hat{A}$; we get

$$
L=\left\{v_{1}\left(\chi_{14} \hat{b}_{4} \hat{a}_{5}\right) \begin{array}{c}
\hat{a} \in \hat{A}, \hat{b} \in \hat{B}, \\
v \in \mathbb{B}(\mathcal{H})_{*}
\end{array}\right\}^{\mathrm{CLS}}=\left\{v_{1}\left(\chi\left(\hat{a}^{\prime} \otimes \hat{b}\right)_{14} \hat{a}_{5}\right) \begin{array}{c}
\hat{a}^{\prime}, \hat{a} \in \hat{A}, \hat{b} \in \hat{B},{ }^{\mathrm{CLS}} \in \mathbb{B}(\mathcal{H})_{*}
\end{array}\right\}^{\mathrm{C}} .
$$

Finally, replacing $\chi(\hat{A} \otimes \hat{B})$ by $\hat{A} \otimes \hat{B}$ gives

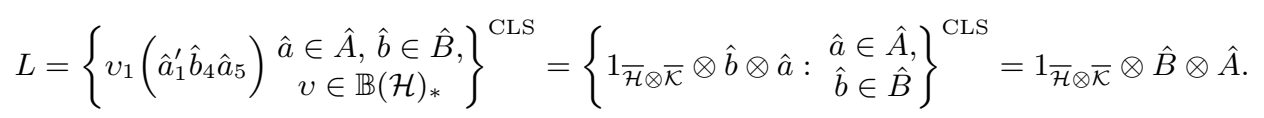

\section{Properties of the generalised quantum codouble}

The general theory of double crossed products 4 says that $\mathfrak{D}^{\chi}(\mathbb{G}, \widehat{\mathbb{H}})^{\wedge}$ contains $\widehat{\mathbb{G}}$ and $\widehat{\mathbb{H}}$ as closed quantum subgroups in the sense of Vaes 41]. Closed quantum subgroups, in the sense of Woronowicz, have been introduced recently in [9. Definition 3.2], using the notion of a $\mathrm{C}^{*}$-algebra generated by a quantum family of multipliers.

Lemma 4.51. $\widehat{\mathbb{G}}$ and $\widehat{\mathbb{H}}$ are closed quantum subgroups of $\mathfrak{D}^{\chi}(\mathbb{G}, \widehat{\mathbb{H}})^{\wedge}$ in the sense of Woronowicz.

Proof. The bicharacter $\widehat{\mathrm{W}}_{\rho 2}^{A} \in \mathcal{U}\left(\mathcal{D}^{\chi} \otimes \hat{A}\right)$ corresponds to a quantum group homomorphism from $\mathfrak{D}^{\chi}(\mathbb{G}, \widehat{\mathbb{H}})^{\wedge}$ to $\widehat{\mathbb{G}}$, and its second leg generates $\widehat{\mathbb{G}}$. Hence $\widehat{\mathbb{G}}$ is a closed quantum subgroup of $\mathfrak{D}^{x}(\mathbb{G}, \widehat{\mathbb{H}})^{\wedge}$ in the sense of Woronowicz. Also, $\widehat{\mathrm{W}}_{\theta 2}^{B} \in \mathcal{U}\left(\mathcal{D}^{\chi} \otimes \hat{B}\right)$ yields a similar conclusion for $\widehat{\mathbb{H}}$.

Lemma 4.51 allows us to decompose every coaction and corepresentation of $\mathfrak{D}^{x}(\mathbb{G}, \widehat{\mathbb{H}})^{\wedge}$ into a pair of coactions and corepresentations of $\widehat{\mathbb{G}}$ and $\widehat{\mathbb{H}}$ with a certain compatibility criterion between them. This becomes more interesting when $\widehat{\mathbb{H}}=\mathbb{G}$, which we shall explore in Section 5.3

Definition 4.52. A $C^{*}$-algebra along with the coactions $\gamma: C \rightarrow C \otimes \hat{A}$ and $\delta: C \rightarrow C \otimes \hat{B}$ of $\widehat{\mathbb{G}}$ and $\widehat{\mathbb{H}}$ is called (right, right) $\chi$-Yetter-Drinfeld if the following diagram commutes:

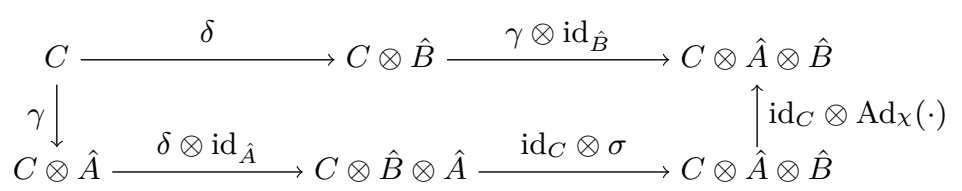

Consider $\hat{A}=\mathrm{C}_{0}(G)$ and $\hat{B}=\mathrm{C}_{0}(H)$ for locally compact groups $G$ and $H$. Then any $G$-C ${ }^{*}$-algebra with trivial $H$-action or any $H$-C $\mathrm{C}^{*}$-algebra with trivial $G$ action makes it Yetter-Drinfeld in this generalised sense. In particular, if we take $\widehat{\mathbb{H}}=\mathbb{G}$ and $\chi=\mathrm{W}^{A}$ then $\mathrm{W}^{A}$-Yetter-Drinfeld-C*-algebras are same as $\mathbb{G}$-YetterDrinfeld $\mathrm{C}^{*}$-algebras defined by Nest and Voigt in 30 . Next we show that $\widehat{\mathcal{D}}^{\chi}$ is a $\chi$-Yetter-Drinfeld algebra using the following lemma. 
Lemma 4.53. The corepresentations $\widehat{\mathrm{W}}_{\rho 2}^{A} \in \mathcal{U}\left(\mathbb{K}\left(\mathcal{H}_{\mathcal{D}}\right) \otimes \hat{A}\right)$ and $\widehat{\mathrm{W}}_{\theta 2}^{B} \in \mathcal{U}\left(\mathbb{K}\left(\mathcal{H}_{\mathcal{D}}\right) \otimes \hat{B}\right)$ of $\widehat{\mathbb{G}}$ and $\widehat{\mathbb{H}}$ on $\mathcal{H}_{\mathcal{D}}$ commute in the following way:

$$
\sigma_{23}^{\chi}\left(\widehat{\mathrm{W}}_{\theta 2}^{B} \widehat{\mathrm{W}}_{\rho 3}^{A}\right)=\widehat{\mathrm{W}}_{\rho 2}^{A} \widehat{\mathrm{W}}_{\theta 3}^{B} \quad \text { in } \mathcal{U}\left(\mathbb{K}\left(\mathcal{H}_{\mathcal{D}}\right) \otimes \hat{A} \otimes \hat{B}\right) .
$$

Proof. This is essentially contained in Lemma 4.452 2:

$$
\sigma_{23}^{\chi}\left(\widehat{\mathrm{W}}_{\theta 2}^{B} \widehat{\mathrm{W}}_{\rho 3}^{A}\right)=\sigma_{12} \sigma_{23}\left(\chi_{12}\left(\mathbb{W}^{B}\right)_{1 \theta}^{*}\left(\mathbb{W}^{A}\right)_{2 \rho}^{*} \chi_{12}^{*}\right)=\sigma_{12} \sigma_{23}\left(\left(\mathbb{W}^{A}\right)_{2 \rho}^{*}\left(\mathbb{W}^{B}\right)_{1 \theta}^{*}\right)=\widehat{\mathrm{W}}_{\rho 2}^{A} \widehat{\mathrm{W}}_{\theta 3}^{B} .
$$

Example 4.54. Define $\Delta_{R}: \widehat{D^{x}} \rightarrow \widehat{D^{x}} \otimes \hat{A}$ as $\Delta_{R}:=\left(\operatorname{id}_{\hat{B}} \otimes \hat{\Delta}_{A}\right)$. Taking Equation $(3.15)$ and the simple computation $\left(\operatorname{id}_{\mathcal{D}^{\chi}} \otimes \Delta_{R}\right)\left(\widehat{\mathrm{W}}_{\theta 2}^{B} \widehat{\mathrm{W}}_{\rho 3}^{A}\right)=\widehat{\mathrm{W}}_{\theta 2}^{B} \widehat{\mathrm{W}}_{\rho 3}^{A} \widehat{\mathrm{W}}_{\rho 4}^{A}$ into account, we conclude that $\Delta_{R}$ is the right quantum group homomorphism corresponding to the bicharacter $\widehat{\mathrm{W}}_{\rho 2}^{A} \in \mathcal{U}\left(\mathcal{D}^{\chi} \otimes \hat{A}\right)$. Similarly, by virtue of Lemma 4.53. $\Delta_{R}^{\prime}: \widehat{\mathcal{D}^{\chi}} \rightarrow \widehat{\mathcal{D}^{\chi}} \otimes \hat{B}$ defined by $\Delta_{R}^{\prime}:=\left(\operatorname{id}_{\hat{B}} \otimes \sigma^{\chi}\right)\left(\hat{\Delta}_{B} \otimes \operatorname{id}_{\hat{A}}\right)$ is the right quantum group homomorphism associated to the bicharacter $\widehat{\mathrm{W}}_{\theta 2}^{B} \in \mathcal{U}\left(\mathcal{D}^{\chi} \otimes \hat{B}\right)$.

Using Lemma 4.53 we compute:

$$
\begin{aligned}
\sigma_{34}^{\chi}\left(\left(\operatorname{id}_{\mathcal{D}} \otimes\left(\left(\Delta_{R}^{\prime} \otimes \operatorname{id}_{\hat{A}}\right) \Delta_{R}\right)\right) \widehat{\mathrm{W}}_{\theta 2}^{B} \widehat{\mathrm{W}}_{\rho 3}^{A}\right) & =\widehat{\mathrm{W}}_{\theta 2}^{B} \sigma_{34}^{\chi}\left(\widehat{\mathrm{W}}_{\rho 3}^{A} \widehat{\mathrm{W}}_{\theta 4}^{B}\right) \widehat{\mathrm{W}}_{\rho 5}^{A} \\
& =\widehat{\mathrm{W}}_{\theta 2}^{B} \widehat{\mathrm{W}}_{\theta 3}^{B} \widehat{\mathrm{W}}_{\rho 4}^{A} \widehat{\mathrm{W}}_{\rho 5}^{A} \\
& =\left(\operatorname{id}_{\mathcal{D}^{x}} \otimes\left(\left(\Delta_{R} \otimes \mathrm{id}_{\hat{A}}\right) \Delta_{R}^{\prime}\right)\right) \widehat{\mathrm{W}}_{\theta 2}^{B} \widehat{\mathrm{W}}_{\rho 3}^{A} .
\end{aligned}
$$

Taking slices on the first leg by functionals on $\mathcal{D}^{\chi}$ shows that $\widehat{\mathcal{D}^{\chi}}$ is a $\chi$-Yetter-Drinfeld $\mathrm{C}^{*}$-algebra.

The next proposition generalises Proposition 3.2 in 30 .

Proposition 4.55. Every $\mathfrak{D}^{\chi}(\mathbb{G}, \widehat{\mathbb{H}})^{\wedge}-\mathrm{C}^{*}$-algebra is also $\chi$-Yetter-Drinfeld $\mathrm{C}^{*}$-algebra, and vice versa.

Proof. Let $C$ be a $\mathfrak{D}^{\chi}(\mathbb{G}, \widehat{\mathbb{H}})^{\wedge}-C^{*}$-algebra. Lemma 2.34 shows that $C$ is contained in $\mathcal{M}\left(C^{\prime} \otimes \widehat{\mathcal{D}^{\chi}}\right)$ for some $\mathrm{C}^{*}$-algebra $C^{\prime}$ with coaction only on $\widehat{\mathcal{D}^{\chi}}$. Now $\widehat{\mathcal{D}^{x}}$ is a $\chi$-Yetter-Drinfeld-C*-algebra. By Lemma $2.34 C$ is also a $\chi$-Yetter-Drinfeld-C $C^{*}$-algebra.

Conversely, let $\gamma: C \rightarrow C \otimes \hat{A}$ and $\delta: C \rightarrow C \otimes \hat{B}$ satisfy 4.31). Define a nondegenerate, injective ${ }^{*}$-homomorphism $\tilde{\gamma}: C \rightarrow C \otimes \widehat{\mathcal{D}}^{\chi}$ by $\tilde{\gamma}:=\left(\delta \otimes \operatorname{id}_{\hat{A}}\right) \gamma$.

The Podles conditions for $\tilde{\gamma}$ gets induced from those for $\gamma$ and $\delta$ in the following way: $\tilde{\gamma}(C) \cdot\left(1_{C} \otimes \widehat{\mathcal{D}}^{\chi}\right)=$ $\left(\left(\delta \otimes \operatorname{id}_{\hat{A}}\right)\left(\gamma(C) \cdot\left(1_{C} \otimes \hat{A}\right)\right) \cdot\left(1_{C} \otimes \hat{B} \otimes 1_{\hat{A}}\right)=\left(\delta(C) \cdot\left(1_{C} \otimes \hat{B}\right)\right) \otimes \hat{A}=C \otimes \widehat{\mathcal{D}}^{\chi}\right.$.

The following computation yields $(2.22)$ for $\tilde{\gamma}$ :

$$
\begin{aligned}
\left(\tilde{\gamma} \otimes \operatorname{id}_{\hat{B} \otimes \hat{A}}\right) \tilde{\gamma}=\left(\delta \otimes \operatorname{id}_{\hat{A} \otimes \hat{B} \otimes \hat{A}}\right)\left(\left(\gamma \otimes \operatorname{id}_{\hat{B}}\right) \delta \otimes \operatorname{id}_{\hat{A}}\right) \gamma & \left.=\sigma_{34}^{\chi}\left(\left(\delta \otimes \operatorname{id}_{\hat{B}}\right) \delta \otimes \operatorname{id}_{\hat{A} \otimes \hat{A}}\right)\left(\gamma \otimes \operatorname{id}_{\hat{A}}\right) \gamma\right) \\
& =\sigma_{34}^{\chi}\left(\left(\operatorname{id}_{C} \otimes \hat{\Delta}_{B} \otimes \hat{\Delta}_{A}\right)\left(\delta \otimes \operatorname{id}_{\hat{A}}\right) \gamma\right) \\
& =\left(\operatorname{id}_{C} \otimes \hat{\Delta}_{\widehat{D}^{\chi}}\right) \tilde{\gamma}
\end{aligned}
$$




\section{Chapter 5}

\section{Braidings for quasitriangular $\mathrm{C}^{*}$-quantum groups}

The twisted tensor product of $\mathrm{C}^{*}$-algebras, in general, does not carry any nontrivial symmetry. Therefore, for a general bicharacter $\chi$, associativity of the functor $\nabla_{\chi}$ makes no sense. Motivated by Hopf algebra theory (see [11, Section 10]), we formulate the notion of unitary $R$-matrix for $C^{*}$-quantum groups in Section 5.1 Thereafter, in Section 5.2 , we define quasitriangular quantum groups and show that their corepresentation categories are braided monoidal. Next we use this to show that the coaction categories of quasitriangular quantum groups are monoidal. In [2, Section 8], Baaj and Skandalis have constructed $R$-matrices on the quantum codoubles of regular quantum groups. We show that the quantum codoubles of quantum groups (not necessarily regular) are quasitriangular, in Section 5.3 Crossed products for a quantum codouble get simpler in terms of Yetter-Drinfeld $\mathrm{C}^{*}$-algebras as shown by Nest and Voigt in [30. We show that their construction implicitly uses the quasitriangularity of the quantum codouble. In Section 5.3.3. we introduce the notion of weak regularity for a quantum group $\mathbb{G}=\left(A, \Delta_{A}\right)$ requiring the natural inner coaction of $\widehat{\mathbb{G}}$ on $A$, implemented by the reduced bicharacter, to satisfy the Podleś condition. Then $A$ becomes a $\mathbb{G}$-Yetter-Drinfeld $\mathrm{C}^{*}$-algebra. Finally, we discuss associativity of crossed products in this context.

\section{$5.1 \quad$ R-matrices}

Let $\mathbb{G}=\left(A, \Delta_{A}\right)$ be a quantum group and let $\mathrm{W} \in \mathcal{U}(\hat{A} \otimes A)$ be its reduced bicharacter.

Definition 5.1. A bicharacter $\mathcal{R} \in \mathcal{U}(A \otimes A)$ is called $R$-matrix if it satisfies:

$$
\mathcal{R}\left(\sigma \circ \Delta_{A}(a)\right) \mathcal{R}^{*}=\Delta_{A}(a) \quad \text { for all } a \in A .
$$

Lemma 5.2. The dual $\widehat{\mathcal{R}}:=\sigma\left(\mathcal{R}^{*}\right) \in \mathcal{U}(A \otimes A)$ of a given bicharacter $\mathcal{R} \in \mathcal{U}(A \otimes A)$ is an $R$-matrix if and only if $\mathcal{R}$ is an $R$-matrix.

Remark 5.3. Condition 5.1 is opposite to the one which is standard for the Hopf algebraists: $\mathcal{R}(\Delta(a)) \mathcal{R}^{*}=$ $\sigma \circ \Delta(a)$. This is because the bicharacter conditions in Definition 3.1 and the conditions used for Hopf algebras differ by taking coopposite comultiplications. We can match Definition 5.1 of an $R$-matrix with the standard one used by Hopf algebraists if we replace $\Delta$ by $\Delta^{\text {cop }}:=\sigma \circ \Delta$.

\subsubsection{Universal lift of R-matrices}

In order to simplify proofs later we shall lift the universal $R$-matrix $\mathcal{R} \in \mathcal{U}(A \otimes A)$ to a unitary element of the multiplier algebra of $A^{\mathrm{u}} \otimes A^{\mathrm{u}}$. 
Proposition 5.4. There is a unique $\mathcal{R}^{\mathrm{u}} \in \mathcal{U}\left(A^{\mathrm{u}} \otimes A^{\mathrm{u}}\right)$ such that

$$
\begin{aligned}
(\Lambda \otimes \Lambda) \mathcal{R}^{\mathrm{u}} & =\mathcal{R} & & \text { in } \mathcal{U}(A \otimes A), \\
\left(\Delta_{A}^{\mathrm{u}} \otimes \mathrm{id}_{A^{\mathrm{u}}}\right) \mathcal{R}^{\mathrm{u}} & =\mathcal{R}_{23}^{\mathrm{u}} \mathcal{R}_{13}^{\mathrm{u}} & & \text { in } \mathcal{U}\left(A^{\mathrm{u}} \otimes A^{\mathrm{u}} \otimes A^{\mathrm{u}}\right), \\
\left(\operatorname{id}_{A^{\mathrm{u}}} \otimes \Delta_{A}^{\mathrm{u}}\right) \mathcal{R}^{\mathrm{u}} & =\mathcal{R}_{12}^{\mathrm{u}} \mathcal{R}_{13}^{\mathrm{u}} & & \text { in } \mathcal{U}\left(A^{\mathrm{u}} \otimes A^{\mathrm{u}} \otimes A^{\mathrm{u}}\right) .
\end{aligned}
$$

Moreover, $\mathcal{R}^{\mathrm{u}}$ satisfies

$$
\mathcal{R}^{\mathrm{u}}\left(\sigma \circ \Delta_{A}^{\mathrm{u}}\left(a^{\mathrm{u}}\right)\right)\left(\mathcal{R}^{\mathrm{u}}\right)^{*}=\Delta_{A}^{\mathrm{u}}\left(a^{\mathrm{u}}\right) \quad \text { for all } a^{\mathrm{u}} \in A^{\mathrm{u}} .
$$

Proof. Proposition 3.16 proves that there is a unique $\mathcal{R}^{\mathrm{u}} \in \mathcal{U}\left(A^{\mathrm{u}} \otimes A^{\mathrm{u}}\right)$ satisfying (5.2)-(5.4).

The nontrivial part is to show that $\mathcal{R}^{\mathrm{u}}$ satisfies $[5.5)$. By 2.42$\}\left\{\left(\omega \otimes \mathrm{id}_{A^{\mathrm{u}}}\right) \mathcal{V} \mid \omega \in \hat{A}^{\prime}\right\}$ is dense in $A^{\mathrm{u}}$, and $\mathcal{V} \in \mathcal{U}\left(\hat{A} \otimes A^{\mathrm{u}}\right)$ is a character in the second leg by 2.41). Therefore, (5.5) is equivalent to:

$$
\mathcal{R}_{23}^{\mathrm{u}} \mathcal{V}_{13} \mathcal{V}_{12}\left(\mathcal{R}_{23}^{\mathrm{u}}\right)^{*}=\mathcal{V}_{12} \mathcal{V}_{13} \quad \text { in } \mathcal{U}\left(\hat{A} \otimes A^{\mathrm{u}} \otimes A^{\mathrm{u}}\right)
$$

We shall prove this in two steps, using similar technique as in the proof of Theorem 4.23 . Consider the following auxiliary bicharacter $\overline{\mathcal{R}}=\left(\Lambda \otimes \mathrm{id}_{C^{\mathrm{u}}}\right) \mathcal{R}^{\mathrm{u}}$ as a unitary in $\mathcal{M}\left(A \otimes A^{\mathrm{u}}\right)$.

The following computation shows that

$$
X:=\mathrm{W}_{12}^{*} \overline{\mathcal{R}}_{23} \mathcal{V}_{13} \mathrm{~W}_{12} \quad \text { in } \mathcal{U}\left(\hat{A} \otimes A \otimes A^{\mathrm{u}}\right)
$$

is a character in the third leg:

$$
\begin{aligned}
\left(\mathrm{id}_{\hat{A}} \otimes \mathrm{id}_{A} \otimes \Delta_{A}^{\mathrm{u}}\right) \mathrm{W}_{12}^{*} \overline{\mathcal{R}}_{23} \mathcal{V}_{13} \mathrm{~W}_{12}=\mathrm{W}_{12}^{*} \overline{\mathcal{R}}_{23} \overline{\mathcal{R}}_{24} \mathcal{V}_{13} \mathcal{V}_{14} \mathrm{~W}_{12} & =\left(\mathrm{W}_{12}^{*} \overline{\mathcal{R}}_{23} \mathcal{V}_{13} \mathrm{~W}_{12}\right)\left(\mathrm{W}_{12}^{*} \overline{\mathcal{R}}_{24} \mathcal{V}_{14} \mathrm{~W}_{12}\right) \\
& =X_{123} X_{124} .
\end{aligned}
$$

The first step follows from 2.41, the second step uses commutation of $\overline{\mathcal{R}}_{24}$ and $\mathcal{V}_{13}$, and the last step is trivial.

A similar routine computation, using [2.41], shows that

$$
Y:=\mathcal{V}_{13} \overline{\mathcal{R}}_{23} \quad \text { in } \mathcal{U}\left(\hat{A} \otimes A \otimes A^{\mathrm{u}}\right)
$$

is a character in the third leg, that is, $\left(\mathrm{id}_{\hat{A}} \otimes \mathrm{id}_{A} \otimes \Delta_{A}^{\mathrm{u}}\right) Y=Y_{123} Y_{124}$.

Using 2.8 we rewrite (5.1) as

$$
\mathcal{R}_{23} \mathrm{~W}_{13} \mathrm{~W}_{12}=\mathrm{W}_{12} \mathrm{~W}_{13} \mathcal{R}_{23} \quad \text { in } U \mathcal{M}(\hat{A} \otimes A \otimes A) .
$$

Thus $\left(\operatorname{id}_{\hat{A}} \otimes \operatorname{id}_{A} \otimes \Lambda\right) X=\left(\operatorname{id}_{\hat{A}} \otimes \operatorname{id}_{A} \otimes \Lambda\right) Y$. Lemma 2.41 gives $X=Y$ or, equivalently,

$$
\mathrm{W}_{12}^{*} \overline{\mathcal{R}}_{23} \mathcal{V}_{13} \mathrm{~W}_{12}=\mathcal{V}_{13} \overline{\mathcal{R}}_{23} \quad \text { in } \mathcal{U}\left(\hat{A} \otimes A \otimes A^{\mathrm{u}}\right) \text {. }
$$

Similarly, using 2.41, we can show that the unitaries $\widetilde{X}:=\mathcal{V}_{13}^{*}\left(\mathcal{R}_{23}^{\mathrm{u}}\right)^{*} \mathcal{V}_{12} \mathcal{V}_{13}$ and $\tilde{Y}:=\mathcal{V}_{12}\left(\mathcal{R}_{23}^{\mathrm{u}}\right)^{*}$ in $\mathcal{U}\left(\hat{A} \otimes A^{\mathrm{u}} \otimes A^{\mathrm{u}}\right)$ are characters in the second leg.

Equation (5.8) shows $\left(\operatorname{id}_{\hat{C}} \otimes \Lambda \otimes \mathrm{id}_{C}\right) \widetilde{X}=\mathcal{V}_{13}^{*} \overline{\mathcal{R}}_{23}^{*} \mathrm{~W}_{12} \mathcal{V}_{13}=\mathrm{W}_{12} \overline{\mathcal{R}}_{23}^{*}=\left(\operatorname{id}_{\hat{C}} \otimes \Lambda \otimes \mathrm{id}_{C}\right) \widetilde{Y}$. Lemma 2.41 gives $\widetilde{X}=\widetilde{Y}$, which is equivalent to 5.6 .

Lemma 5.5. The unitary $\mathcal{R}^{\mathrm{u}} \in \mathcal{U}\left(A^{\mathrm{u}} \otimes A^{\mathrm{u}}\right)$ in Proposition 5.4 satisfies

$$
\begin{aligned}
\left(e^{\mathrm{u}} \otimes \mathrm{id}_{A^{\mathrm{u}}}\right) \mathcal{R}^{\mathrm{u}} & =\left(\mathrm{id}_{A^{\mathrm{u}}} \otimes e^{\mathrm{u}}\right) \mathcal{R}^{\mathrm{u}}=1_{A^{\mathrm{u}}}, \\
\mathcal{R}_{12}^{\mathrm{u}} \mathcal{R}_{13}^{\mathrm{u}} \mathcal{R}_{23}^{\mathrm{u}} & =\mathcal{R}_{23}^{\mathrm{u}} \mathcal{R}_{13}^{\mathrm{u}} \mathcal{R}_{12}^{\mathrm{u}} \quad \text { in } \mathcal{U}\left(A^{\mathrm{u}} \otimes A^{\mathrm{u}} \otimes A^{\mathrm{u}}\right) .
\end{aligned}
$$

Proof. We apply $\mathrm{id}_{A^{\mathrm{u}}} \otimes e^{\mathrm{u}} \otimes \mathrm{id}_{A^{\mathrm{u}}}$ to 5.3 and 5.4 and use 2.45 for $e^{\mathrm{u}}$ to get:

$$
\mathcal{R}^{\mathrm{u}}=\left(\left(e^{\mathrm{u}} \otimes \mathrm{id}_{C^{\mathrm{u}}}\right) \mathcal{R}^{\mathrm{u}}\right)_{23} \mathcal{R}^{\mathrm{u}}=\left(\left(\operatorname{id}_{C^{\mathrm{u}}} \otimes e^{\mathrm{u}}\right) \mathcal{R}^{\mathrm{u}}\right)_{12} \mathcal{R}^{\mathrm{u}} .
$$

Since $R$ is unitary, this yields both $\left(e^{\mathrm{u}} \otimes \operatorname{id}_{C^{\mathrm{u}}}\right) \mathcal{R}^{\mathrm{u}}=1_{A^{\mathrm{u}}}$ and $\left(\operatorname{id}_{C^{\mathrm{u}}} \otimes e^{\mathrm{u}}\right) \mathcal{R}^{\mathrm{u}}=1_{A^{\mathrm{u}}}$.

The following computation yields 5.10 :

$$
\mathcal{R}_{12}^{\mathrm{u}} \mathcal{R}_{13}^{\mathrm{u}} \mathcal{R}_{23}^{\mathrm{u}}=\left(\left(\operatorname{id}_{C^{\mathrm{u}}} \otimes \Delta^{\mathrm{u}}\right) \mathcal{R}^{\mathrm{u}}\right) \mathcal{R}_{23}^{\mathrm{u}}=\mathcal{R}_{23}^{\mathrm{u}}\left(\left(\operatorname{id}_{C^{\mathrm{u}}} \otimes \sigma \circ \Delta^{\mathrm{u}}\right) \mathcal{R}^{\mathrm{u}}\right)=\mathcal{R}_{23}^{\mathrm{u}} \mathcal{R}_{13}^{\mathrm{u}} \mathcal{R}_{12}^{\mathrm{u}},
$$

where the first and third step use 5.4 and the second step uses 5.5. 


\subsection{Quasitriangular $\mathrm{C}^{*}$-quantum groups}

In a purely algebraic framework it is customary to let Hopf algebras act on vector spaces. The representation category of a quasitriangular Hopf algebra ([11], 12] ) is a braided monoidal category. In the $\mathrm{C}^{*}$-algebraic framework, we use corepresentations (equivalently, representation of the universal dual). This justifies the following definition:

Definition 5.6. A $C^{*}$-quantum group $\mathbb{G}=\left(A, \Delta_{A}\right)$ is quasitriangular when equipped with an $R$-matrix $\mathcal{R} \in \mathcal{U}(\hat{A} \otimes \hat{A})$

\subsubsection{The corepresentation category}

Let us briefly recall the discussion from Sections 3.1 and 3.2 in [39] on the categorical structure of corepresentations of quantum groups in general.

Let $\mathrm{U}^{\mathcal{H}_{1}} \in \mathcal{U}\left(\mathbb{K}\left(\mathcal{H}_{1}\right) \otimes A\right)$ and $\mathrm{U}^{\mathcal{H}_{2}} \in \mathcal{U}\left(\mathbb{K}\left(\mathcal{H}_{2}\right) \otimes A\right)$ be corepresentations of $\mathbb{G}$ on the Hilbert spaces $\mathcal{H}_{1}$ and $\mathcal{H}_{2}$. An element $t \in \mathbb{B}\left(\mathcal{H}_{1}, \mathcal{H}_{2}\right)$ is an inertwiner between $\mathrm{U}^{\mathcal{H}_{1}}$ and $\mathrm{U}^{\mathcal{H}_{2}}$ if and only if $\left(t \otimes 1_{A}\right) \mathrm{U}^{\mathcal{H}_{1}}=$ $\mathrm{U}^{\mathcal{H}_{2}}\left(t \otimes 1_{A}\right)$. The set of all operators intertwining $\mathrm{U}^{\mathcal{H}_{1}}$ and $\mathrm{U}^{\mathcal{H}_{2}}$ is denoted by $\operatorname{Hom}\left(\mathrm{U}^{\mathcal{H}_{1}}, \mathrm{U}^{\mathcal{H}_{2}}\right)$.

Notation 5.7. $\operatorname{Corep}(\mathbb{G})$ denotes the category where objects are the corepresentations of $\mathbb{G}$ and morphisms are intertwiners.

Furthermore,

$$
\mathrm{U}^{\mathcal{H}_{1}} \oplus \mathrm{U}^{\mathcal{H}_{2}}:=\mathrm{U}_{13}^{\mathcal{H}_{1}} \mathrm{U}_{23}^{\mathcal{H}_{2}} \quad \text { in } \mathcal{U}\left(\mathbb{K}\left(\mathcal{H}_{1} \otimes \mathcal{H}_{2}\right) \otimes A\right) .
$$

is again a corepresentation of $\mathbb{G}$ on $\mathcal{H}_{1} \otimes \mathcal{H}_{2}$.

Definition 5.8 (see $39,3.3 .2]$ ). $\mathrm{U}^{\mathcal{H}_{1}} \odot \mathrm{U}^{\mathcal{H}_{2}}$ is the tensor product of the corepresentations $\mathrm{U}^{\mathcal{H}_{1}}$ and $\mathrm{U}^{\mathcal{H}_{2}}$.

The following routine computation shows that $(1)$ is associative:

$$
\mathrm{U}^{\mathcal{H}_{1}} \oplus\left(\mathrm{U}^{\mathcal{H}_{2}} \oplus \mathrm{U}^{\mathcal{H}_{3}}\right)=\mathrm{U}_{14}^{\mathcal{H}_{1}} \oplus\left(\mathrm{U}_{13}^{\mathcal{H}_{2}} \mathrm{U}_{23}^{\mathcal{H}_{2}}\right)=\mathrm{U}_{14}^{\mathcal{H}_{1}} \mathrm{U}_{24}^{\mathcal{H}_{2}} \mathrm{U}_{34}^{\mathcal{H}_{2}}=\left(\mathrm{U}_{14}^{\mathcal{H}_{1}} \mathrm{U}_{24}^{\mathcal{H}_{2}}\right) \oplus \mathrm{U}^{\mathcal{H}_{3}}=\left(\mathrm{U}^{\mathcal{H}_{1}} \oplus \mathrm{U}^{\mathcal{H}_{2}}\right) \oplus \mathrm{U}^{\mathcal{H}_{3}}
$$

Thus $\operatorname{Corep}(\mathbb{G})$ is a monoidal category ${ }^{1}$

Let $\Sigma^{\left(\mathcal{H}_{1}, \mathcal{H}_{2}\right)}: \mathcal{H}_{1} \otimes \mathcal{H}_{2} \rightarrow \mathcal{H}_{2} \otimes \mathcal{H}_{1}$ be the flip operator. As already pointed out in $39, \Sigma_{12}^{\left(\mathcal{H}_{1}, \mathcal{H}_{2}\right)} \in$ $\operatorname{Hom}\left(\left(\mathrm{U}^{\mathcal{H}_{1}} \oplus \mathrm{U}^{\mathcal{H}_{2}}, \mathrm{U}^{\mathcal{H}_{2}} \oplus \mathrm{U}^{\mathcal{H}_{1}}\right)\right.$ if and only if $\mathbb{G}$ is commutative. Hence $\Sigma^{(\cdot, \cdot)}$ does not endow $\mathfrak{C o r e p}(\mathbb{G})$ with a braiding in general.

Let $\mathrm{U}^{\mathcal{H}_{i}} \in \mathcal{U}\left(\mathbb{K}\left(\mathcal{H}_{i}\right) \otimes A\right)$ be corepresentation of $\mathbb{G}$ on $\mathcal{H}_{i}$ for $i=1,2$. Let $\mathcal{R}^{\mathrm{u}} \in \mathcal{U}\left(\hat{A}^{\mathrm{u}} \otimes \hat{A}^{\mathrm{u}}\right)$ be the universal lift (constructed in Proposition 5.4) of $\mathcal{R} \in \mathcal{U}(\hat{A} \otimes \hat{A})$. By the universal property (2.34) of $\tilde{\mathcal{V}} \in \mathcal{U}\left(\hat{A}^{\mathrm{u}} \otimes A\right)$, there are unique $\hat{\varphi}_{i} \in \operatorname{Mor}\left(\hat{A}^{\mathrm{u}}, \mathbb{K}\left(\mathcal{H}_{i}\right)\right)$ such that $\left(\hat{\varphi}_{i} \otimes \operatorname{id}_{A}\right) \tilde{\mathcal{V}}=\mathrm{U}^{\mathcal{H}_{i}}$ for $i=1,2$. Define the unitaries $\chi^{\mathcal{H}_{1}}: \mathcal{H}_{1} \otimes \mathcal{H}_{2} \rightarrow \mathcal{H}_{2} \otimes \mathcal{H}_{1}$ by

$$
\begin{aligned}
& \mathrm{Z}^{\left(\mathcal{H}_{1}, \mathcal{H}_{2}\right)}:=\left(\hat{\varphi}_{1} \otimes \hat{\varphi}_{2}\right)\left(\mathcal{R}^{\mathrm{u}}\right)^{*} \quad \text { in } \mathcal{U}\left(\mathcal{H}_{1} \otimes \mathcal{H}_{2}\right),
\end{aligned}
$$

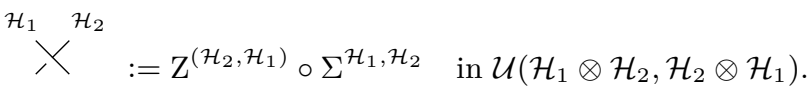

Proposition 5.9. The unitaries $\nearrow^{\mathcal{H}_{1}}: \mathcal{H}_{1} \otimes \mathcal{H}_{2} \rightarrow \mathcal{H}_{2} \otimes \mathcal{H}_{1}$ are $\mathbb{G}$-equivariant, that is,

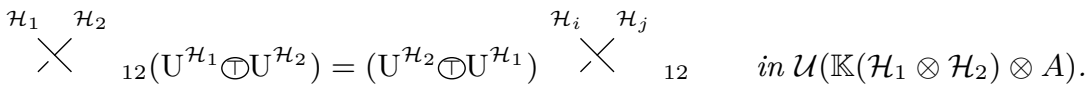

for all $\mathrm{U}^{\mathcal{H}_{1}}, \mathrm{U}^{\mathcal{H}_{2}} \in \operatorname{Corep}(\mathbb{G})$.

They define a braiding on $\operatorname{Corep}(\mathbb{G})$, that is, the following hexagons commute for all $\mathrm{U}^{\mathcal{H}_{i}} \in \mathfrak{C o r e p}(\mathbb{G})$, $i=1,2,3$ :

\footnotetext{
${ }^{1}$ More precisely, $\mathfrak{C o r e p}(\mathbb{G})$ is a monoidal $\mathrm{W}^{*}$-category. See 15 for the general theory of $\mathrm{W}^{*}$-category.
} 


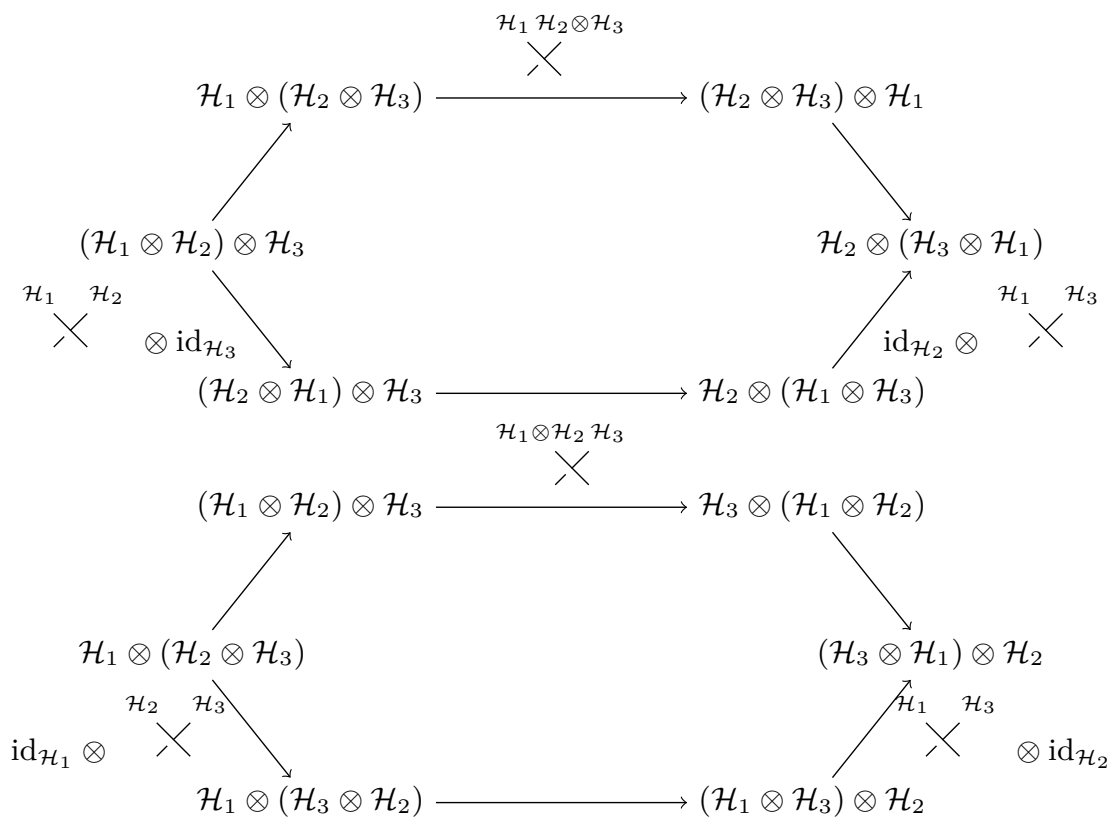

the unlabelled arrows are associators.

Proof. By the universal property 2.34 and the character condition 2.35 of $\tilde{\mathcal{V}}$, the corepresentations $\mathrm{U}^{\mathcal{H}_{1}} \oplus \mathrm{U}^{\mathcal{H}_{2}}$ are characterised by the representations $\left(\hat{\phi}_{1} \otimes \hat{\phi}_{2}\right) \circ \sigma \circ \hat{\Delta}_{A}^{\mathrm{u}}$ of $\hat{A}^{\mathrm{u}}$ on $\mathcal{H}_{1} \otimes \mathcal{H}_{2}$ :

$$
\left(\left(\hat{\phi}_{1} \otimes \hat{\phi}_{2}\right) \circ \sigma \circ \hat{\Delta}_{A}^{\mathrm{u}} \otimes \mathrm{id}_{A}\right) \tilde{\mathcal{V}}=\mathrm{U}^{\mathcal{H}_{1}} \oplus \mathrm{U}^{\mathcal{H}_{2}} .
$$

The following computation yields (5.14) :

$$
\begin{aligned}
\bigwedge^{\mathcal{H}_{1}}{ }^{\mathcal{H}_{2}}\left(\mathrm{U}^{\mathcal{H}_{1}} \oplus \mathrm{U}^{\mathcal{H}_{2}}\right) & =\left(\hat{\phi}_{2} \otimes \hat{\phi}_{1} \otimes \mathrm{id}_{A}\right)\left(\left(\mathcal{R}_{12}^{\mathrm{u}}\right)^{*}\left(\hat{\Delta}_{A}^{\mathrm{u}} \otimes \mathrm{id}_{A}\right) \tilde{\mathcal{V}}\right) \Sigma_{12}^{\left(\mathcal{H}_{1}, \mathcal{H}_{2}\right)} \\
& \left.=\left(\left(\left(\hat{\phi}_{2} \otimes \hat{\phi}_{1}\right) \circ \sigma \circ \hat{\Delta}_{A}^{\mathrm{u}}\right) \otimes \mathrm{id}_{A}\right) \tilde{\mathcal{V}}\right) \mathrm{Z}_{12}^{\left(\mathcal{H}_{2}, \mathcal{H}_{1}\right)} \Sigma^{\left(\mathcal{H}_{1}, \mathcal{H}_{2}\right)}=\left(\mathrm{U}^{\mathcal{H}_{2}} \odot \mathrm{U}^{\mathcal{H}_{1}}\right) \searrow_{12}^{\mathcal{H}_{1}} \searrow^{\mathcal{H}_{2}}
\end{aligned}
$$

The first equality uses 5.15 and $(5.13)$, the second equality follows from 5.5 and $(5.12$, and the last equality uses 5.15 and (5.13).

By (5.13) and (5.15),

$$
\begin{aligned}
& \mathcal{H}_{1} \mathcal{H}_{2} \otimes \mathcal{H}_{3} \\
& \Upsilon^{\prime}:=\mathrm{Z}^{\left(\mathcal{H}_{2} \otimes \mathcal{H}_{3}, \mathcal{H}_{1}\right)} \Sigma^{\left(\mathcal{H}_{1}, \mathcal{H}_{2} \otimes \mathcal{H}_{3}\right)}=\left(\left(\hat{\phi}_{2} \otimes \hat{\phi}_{3} \otimes \hat{\phi}_{1}\right)\left(\sigma \circ \hat{\Delta}_{A}^{\mathrm{u}} \otimes \mathrm{id}_{\hat{A}^{\mathrm{u}}}\right)\left(\mathcal{R}^{\mathrm{u}}\right)^{*}\right) \circ \Sigma^{\left(\mathcal{H}_{1}, \mathcal{H}_{2} \otimes \mathcal{H}_{3}\right)} .
\end{aligned}
$$

Now we check the first braiding diagram:

$$
\begin{aligned}
& \left(\left(\hat{\phi}_{2} \otimes \hat{\phi}_{3} \otimes \hat{\phi}_{1}\right)\left(\sigma \circ \hat{\Delta}_{A}^{\mathrm{u}} \otimes \mathrm{id}_{\hat{A}^{\mathrm{u}}}\right)\left(\mathcal{R}^{\mathrm{u}}\right)^{*}\right) \circ \Sigma^{\left(\mathcal{H}_{1}, \mathcal{H}_{2} \otimes \mathcal{H}_{3}\right)} \\
& =\left(\left(\hat{\phi}_{2} \otimes \hat{\phi}_{3} \otimes \hat{\phi}_{1}\right)\left(\left(\mathcal{R}^{\mathrm{u}}\right)_{23}^{*}\left(\mathcal{R}^{\mathrm{u}}\right)_{13}^{*}\right)\right) \Sigma_{23}^{\left(\mathcal{H}_{1}, \mathcal{H}_{3}\right)} \Sigma_{12}^{\left(\mathcal{H}_{1}, \mathcal{H}_{2}\right)} \\
& =\mathrm{Z}_{23}^{\left(\mathcal{H}_{3}, \mathcal{H}_{1}\right)} \mathrm{Z}_{13}^{\left(\mathcal{H}_{2}, \mathcal{H}_{1}\right)} \Sigma_{23}^{\left(\mathcal{H}_{1}, \mathcal{H}_{3}\right)} \Sigma_{12}^{\left(\mathcal{H}_{1}, \mathcal{H}_{2}\right)}
\end{aligned}
$$

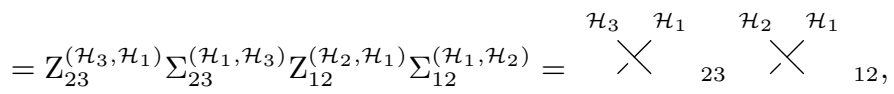

where the first equality uses $(5.3)$, the second equality uses 5.12 , the third equality uses the property of the flip operator $\Sigma$, and the fourth equality follows from $(5.13)$. 
A similar computation for $\aleph^{\mathcal{H}_{1} \otimes \mathcal{H}_{2} \mathcal{H}_{3}}$ yields the second braiding diagram:

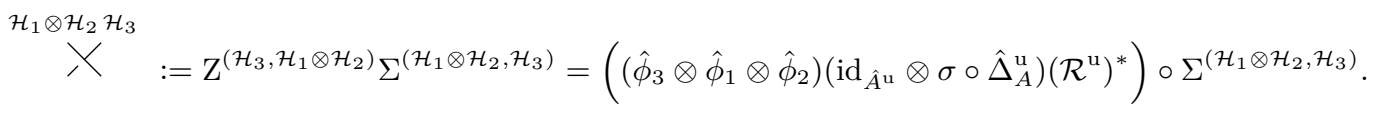

Corollary 5.10. If $\mathbb{C}$ carries the trivial corepresentation of $\mathbb{G}$, then

$$
\mathbb{C}^{\mathcal{H}}: \mathbb{C} \otimes \mathcal{H} \rightarrow \mathcal{H} \otimes \mathbb{C} \quad \text { and } \quad \mathcal{H}^{\mathbb{C}}: \mathcal{H} \otimes \mathbb{C} \rightarrow \mathbb{C} \otimes \mathcal{H}
$$

are the canonical isomorphisms. For any three corepresentations of $\mathbb{G}$,

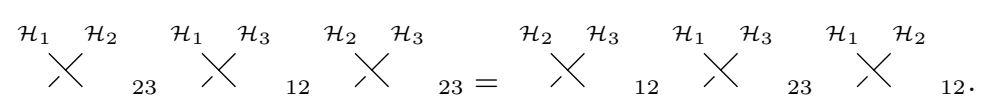

Proof. These are general properties of braided monoidal categories, see 17, Proposition 2.1]. Alternatively, we may deduce these assertions from (5.9), 5.10) and (5.12).

Remark 5.11. Lemma 5.2 shows that the dual of an $R$-matrix $\mathcal{R} \in \mathcal{U}(\hat{A} \otimes \hat{A})$ is again an $R$-matrix.

Following (5.12), we define $\widetilde{\mathrm{Z}^{\left(\mathcal{H}_{1}, \mathcal{H}_{2}\right)}}:=\left(\hat{\varphi}_{1} \otimes \hat{\varphi}_{2}\right)\left(\widehat{\mathcal{R}^{\mathrm{u}}}\right)^{*}=\Sigma^{\mathcal{H}_{2}, \mathcal{H}_{1}} \circ\left(\mathrm{Z}^{\left(\mathcal{H}_{2}, \mathcal{H}_{1}\right)}\right)^{*} \circ \Sigma^{\mathcal{H}_{1}, \mathcal{H}_{2}}$. Then ${\widehat{\mathcal{H}_{2}}}^{\mathcal{H}_{1}} \in$ $\mathcal{U}\left(\mathcal{H}_{2} \otimes \mathcal{H}_{1}, \mathcal{H}_{1} \otimes \mathcal{H}_{2}\right)$ defined by $\widetilde{\mathcal{H}^{\mathcal{H}_{1}}}:=\mathrm{Z}^{\left(\mathcal{H}_{1}, \mathcal{H}_{2}\right)} \circ \Sigma^{\mathcal{H}_{2}, \mathcal{H}_{1}}=\Sigma^{\mathcal{H}_{2}, \mathcal{H}_{1}} \circ\left(\mathrm{Z}^{\left(\mathcal{H}_{2}, \mathcal{H}_{1}\right)}\right)^{*}$ gives another braiding on $\operatorname{Corep}(\mathbb{G})$.

\subsubsection{Symmetric braidings}

Definition 5.12. An $R$-matrix $\mathcal{R} \in \mathcal{U}(A \otimes A)$ is called antisymmetric if $\mathcal{R}^{*}=\sigma(\mathcal{R})$ for the flip $\sigma: A \otimes A \rightarrow$ $A \otimes A, a_{1} \otimes a_{2} \mapsto a_{2} \otimes a_{1}$.

Lemma 5.13. If $\mathcal{R}$ is antisymmetric, then $\left(\mathcal{R}^{\mathrm{u}}\right)^{*}=\sigma\left(\mathcal{R}^{\mathrm{u}}\right)$ for the universal lift $\mathcal{R}^{\mathrm{u}} \in \mathcal{U}\left(A^{\mathrm{u}} \otimes A^{\mathrm{u}}\right)$ constructed in Proposition 5.4 .

Proof. Both $\sigma\left(\mathcal{R}^{\mathrm{u}}\right)^{*}$ and $\mathcal{R}^{\mathrm{u}}$ are bicharacters that lift $\mathcal{R}$. Since the lifting of bicharacters is unique by Proposition 3.16, they must be equal.

Proposition 5.14. The braiding on the monoidal category of corepresentations of $\mathbb{G}$ on Hilbert spaces constructed in 5.13 is symmetric if and only if $\mathcal{R} \in \mathcal{U}(\hat{A} \otimes \hat{A})$ is antisymmetric.

Proof. Let $\mathcal{H}_{1}$ and $\mathcal{H}_{2}$ be Hilbert spaces with corepresentations of $\mathbb{G}$. Let $\hat{\phi}_{i}: \hat{A}^{\mathrm{u}} \rightarrow \mathbb{B}\left(\mathcal{H}_{i}\right)$ be the corresponding ${ }^{*}$-representations. Then the braiding operator

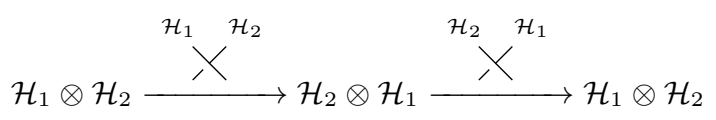

is the unitary $\left(\hat{\phi}_{1} \otimes \hat{\phi}_{2}\right)\left(\mathcal{R}^{\mathrm{u}}\right)^{*} \circ \Sigma^{\left(\mathcal{H}_{2}, \mathcal{H}_{1}\right)} \circ\left(\hat{\phi}_{2} \otimes \hat{\phi}_{1}\right)\left(\mathcal{R}^{\mathrm{u}}\right)^{*} \circ \Sigma^{\left(\mathcal{H}_{1}, \mathcal{H}_{2}\right)}=\left(\hat{\phi}_{1} \otimes \hat{\phi}_{2}\right)\left(\sigma\left(\mathcal{R}^{\mathrm{u}}\right) \mathcal{R}^{\mathrm{u}}\right)^{*}$. This is the identity operator for all representations $\hat{\phi}_{i}$ if and only if $\sigma\left(\mathcal{R}^{\mathrm{u}}\right) \mathcal{R}^{\mathrm{u}}=1$. 


\subsubsection{The abelian case}

Now we consider $R$-matrices for a commutative quantum group $\left(\mathrm{C}_{0}(G), \Delta\right)$ for a locally compact group $G$. Since $\mathrm{C}_{0}(G) \otimes \mathrm{C}_{0}(G)$ is commutative as well, (5.1) simplifies to the condition $\sigma \circ \Delta=\Delta$, which is equivalent to $G$ being commutative. Hence there is no $R$-matrix for nonabelian $G$. In the abelian case, (5.1) holds for any unitary $\mathcal{R} \in \mathcal{U}\left(\mathrm{C}_{0}(G) \otimes \mathrm{C}_{0}(G)\right)$. Thus an $R$-matrix for $\mathbb{G}$ is simply a bicharacter in $\mathrm{C}_{\mathrm{b}}(G \times G)$. Equivalently, $\mathcal{R}$ is a function $\rho: G \times G \rightarrow \mathrm{U}(1)$ satisfying $\rho(x y, z)=\rho(x, z) \rho(y, z)$ and $\rho(x, y z)=\rho(x, y) \rho(x, z)$. Being antisymmetric means $\rho(x, y) \rho(y, x)=1$ for all $x, y \in G$.

Any bicharacter $\rho$ as above is of the form $\rho(x, y)=\langle\hat{\rho}(x), y\rangle$ for a group homomorphism $\hat{\rho}: G \rightarrow \hat{G}$ to the Pontrjagin dual $\hat{G}$, with $\rho(x, \sqcup)=\hat{\rho}$. This is a special case of the interpretation of bicharacters as quantum group homomorphisms in Chapter 3

The map $\hat{\rho}$ has a dual map $\hat{\rho}^{*}: G \cong \hat{\hat{G}} \rightarrow \hat{G}$, defined by $\left\langle x, \hat{\rho}^{*}(y)\right\rangle=\langle\hat{\rho}(x), y\rangle$ for all $x, y \in G$. The bicharacter $\rho$ is antisymmetric if and only if $\hat{\rho}^{*}=\rho^{-1}$.

Now we consider the category of Hilbert space representations of an abelian locally compact group $G$. This is equivalent to the category of corepresentations of $\left(\mathrm{C}_{0}(G), \Delta\right)$ or to the category of representations of $\mathrm{C}^{*}(G) \cong \mathrm{C}_{0}(\hat{G})$. The tensor category of $G$-representations is already symmetric for the obvious braiding $\Sigma(x \otimes y):=y \otimes x$. This corresponds to the $R$-matrix 1. We want to describe the braiding operators from nontrivial $R$-matrices.

A representation of $\mathrm{C}_{0}(\hat{G})$ is classified using a measurable field of Hilbert spaces $\left(\mathcal{H}_{x}\right)_{x \in \hat{G}}$ over $\hat{G}$ and a measure class $[\mu]$ on $\hat{G}$. Let $\int_{\hat{G}}^{\oplus} \mathcal{H}_{x} \mathrm{~d} \mu(x)$ denote the space of $L^{2}$-sections of the measurable field with respect to the measure $\mu$, with $\mathrm{C}_{0}(\hat{G})$ acting by pointwise multiplication. All representations of $\mathrm{C}_{0}(\hat{G})$ are of this form with $\left(\mathcal{H}_{x}\right)$ unique up to isomorphism and $\mu$ unique up to measure equivalence.

Let $\mathcal{H}_{1}=\int_{\hat{G}}^{\oplus} \mathcal{H}_{1_{x}} \mathrm{~d} \mu_{1}(x)$ and $\mathcal{H}_{2}=\int_{\hat{G}}^{\oplus} \mathcal{H}_{2_{x}} \mathrm{~d} \mu_{2}(x)$ be two Hilbert space representations of $G$. Then

$$
\mathcal{H}_{1} \otimes \mathcal{H}_{2}=\int_{\hat{G} \times \hat{G}}^{\oplus} \mathcal{H}_{1_{x}} \otimes \mathcal{H}_{2 y} \mathrm{~d} \mu_{1}(x) \mathrm{d} \mu_{2}(y) .
$$

Since $\mathrm{C}_{0}(\hat{G}) \otimes \mathrm{C}_{0}(\hat{G}) \cong \mathrm{C}_{0}(\hat{G} \times \hat{G})$ acts by pointwise multiplication, we have

$$
\stackrel{\mathcal{H}}{1}^{\mathcal{H}_{2}}\left(\int_{\hat{G}^{2}} \xi_{x, y} \mathrm{~d} \mu_{1}(x) \mathrm{d} \mu_{2}(y)\right)=\int_{\hat{G}^{2}} \rho(x, y) \Sigma \xi_{x, y} \mathrm{~d} \mu_{1}(x) \mathrm{d} \mu_{2}(y) .
$$

Example 5.15. Consider $G=\mathbb{Z} / 2=\{ \pm 1\}$ and let $\rho(x, y)=x y \in \mathbb{Z} / 2 \subseteq \mathrm{U}(1)$; this bicharacter corresponds to the isomorphism $G \cong \hat{G}$. The spectral analysis above writes a $\mathbb{Z} / 2$-Hilbert space as a $\mathbb{Z} / 2$-graded Hilbert space, splitting it into even and odd elements with respect to the action of the generator in $\mathbb{Z} / 2$. The braiding unitary on $\xi \otimes \eta$ is $\Sigma$ if $\xi$ or $\eta$ is even, and $-\Sigma$ if both $\xi$ and $\eta$ are odd. This is the usual Koszul sign rule.

Thus standard operations with $\mathbb{Z} / 2$-graded Hilbert spaces and $\mathrm{C}^{*}$-algebras are special cases of our more general constructions for quasitriangular $\mathrm{C}^{*}$-quantum groups.

\subsubsection{Associative crossed product of $\mathrm{C}^{*}$-algebras}

Let $\mathbb{G}=\left(A, \Delta_{A}\right)$ be a quasitriangular quantum group with $R$-matrix $\mathcal{R} \in \mathcal{U}(\hat{A} \otimes \hat{A})$. The main result of this section is to induce a monoidal structure on $\mathfrak{C}^{*} \mathfrak{a l g}(\mathbb{G})$ using the braided monoidal structure of $\mathfrak{C o r e p}(\mathbb{G})$. As $\otimes_{\mathcal{R}}$ is a special case of the general construction of $\otimes$ discussed in Chapter 4 it satisfies all the properties of $\otimes$ in general. We shall devote the rest of this section to the following main result.

Theorem 5.16. $\mathfrak{C}^{*} \mathfrak{a l g}(\mathbb{G})$ with the tensor product $\bigotimes_{\mathcal{R}}$ is a monoidal category.

Let $\mathrm{W} \in \mathcal{U}(\hat{A} \otimes A)$ be the reduced bicharacter of $\mathbb{G}$. By Definition 4.5 , a pair of representations $(\alpha, \beta)$ of $A$ on a Hilbert space $\mathcal{H}$ is called an $\mathcal{R}$-Heisenberg pair if:

$$
\mathrm{W}_{1 \alpha} \mathrm{W}_{2 \beta}=\mathrm{W}_{2 \beta} \mathrm{W}_{1 \alpha} \mathcal{R}_{12} \quad \text { in } \mathcal{U}(\hat{A} \otimes \hat{A} \otimes \mathbb{K}(\mathcal{H})) .
$$

Let $(C, \gamma),(D, \delta)$ be $\mathbb{G}$-C $\mathrm{C}^{*}$-algebras and construct the crossed product $\left(C \nabla_{\mathcal{R}} D, \iota_{C}, \iota_{D}\right)$ as in Lemma 4.16 Moreover, let $\left(\varphi, \mathrm{U}^{\mathcal{H}}\right)$ and $\left(\psi, \mathrm{U}^{\mathcal{K}}\right)$ be faithful covariant representations of $(C, \gamma, A)$ and $(D, \delta, \psi)$ on the Hilbert spaces $\mathcal{H}$ and $\mathcal{K}$, respectively. 
Theorem 5.17. There is a unique coaction $\gamma \bowtie_{\mathcal{R}} \delta$ of $\mathbb{G}$ on $C \bigotimes_{\mathcal{R}} D$ such that $\left(\varphi \bigotimes_{\mathcal{R}} \psi, \mathrm{U}^{\mathcal{H}} \oplus \mathrm{U}^{\mathcal{K}}\right)$ is a covariant representation of $\left(C \otimes_{\mathcal{R}} D, \gamma \bowtie_{\mathcal{R}} \delta, A\right)$ on $\mathcal{H} \otimes \mathcal{K}$.

Proof. By Theorem 4.23 for any $\mathcal{R}$-Heisenberg pair $(\alpha, \beta)$ acting on $\mathcal{L}$ we have

$$
\mathrm{U}_{1 \alpha}^{\mathcal{H}} \mathrm{U}_{2 \beta}^{\mathcal{K}} \mathrm{Z}_{12}^{(\mathcal{H}, \mathcal{K})}=\mathrm{U}_{2 \beta}^{\mathcal{K}} \mathrm{U}_{1 \alpha}^{\mathcal{H}} \quad \text { in } \mathcal{U}(\mathcal{H} \otimes \mathcal{K} \otimes \mathcal{L}),
$$

where $\mathrm{Z}^{(\mathcal{H}, \mathcal{K})}$ is defined in 5.12 . Then ${ }^{\mathcal{K}} \chi^{\mathcal{H}}:=\mathrm{Z}^{(\mathcal{H}, \mathcal{K})} \circ \Sigma^{\mathcal{K}, \mathcal{H}}$ is the corresponding braiding operator.

By Theorem 4.24 there is a faithful representation $\varphi \bigotimes_{\mathcal{R}} \psi: C \otimes_{\mathcal{R}} D \rightarrow \mathbb{B}(\mathcal{H} \otimes \mathcal{K})$ such that

$$
\begin{array}{lll}
\left(\varphi \bigotimes_{\mathcal{R}} \psi\right) \circ \iota_{C}(c)=\varphi(c) \otimes 1_{K} & \text { for all } c \in C, \\
\left(\varphi \bigotimes_{\mathcal{R}} \psi\right) \circ \iota_{D}(d)=\mathcal{K}^{\mathcal{K}}\left(\psi(d) \otimes 1_{\mathcal{H}}\right)\left(\chi^{\mathcal{H}}\right)^{*} & \text { for all } d \in D .
\end{array}
$$

We compute:

$$
\begin{aligned}
& \left(\mathrm{U}^{\mathcal{H}} \oplus \mathrm{U}^{\mathcal{K}}\right)\left(\left(\varphi \nabla_{\mathcal{R}} \psi\right)\left(\iota_{C}(c) \iota_{D}(d)\right) \otimes 1_{A}\right)\left(\mathrm{U}^{\mathcal{H}} \oplus \mathrm{U}^{\mathcal{K}}\right)^{*}
\end{aligned}
$$

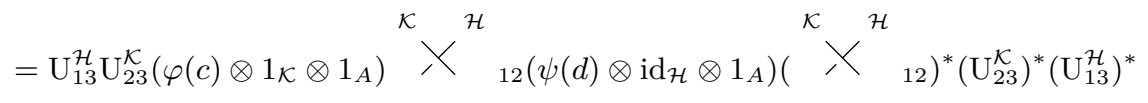

$$
\begin{aligned}
& =\mathrm{U}_{13}^{\mathcal{H}} \mathrm{U}_{23}^{\mathcal{K}}\left(\varphi(c) \otimes 1_{\mathcal{K}} \otimes 1_{A}\right) \aleph^{\mathcal{K}} \aleph^{\mathcal{H}}\left(\psi(d) \otimes \mathrm{id}_{\mathcal{H}} \otimes 1_{A}\right)\left(\mathrm{U}_{23}^{\mathcal{H}}\right)^{*}\left(\mathrm{U}_{13}^{\mathcal{K}}\right)^{*}\left(\aleph^{\mathcal{K}}\right)_{12}^{*} \\
& =\mathrm{U}_{13}^{\mathcal{H}}\left(\varphi(c) \otimes 1_{\mathcal{K}} \otimes 1_{A}\right)\left(\mathrm{U}_{13}^{\mathcal{H}}\right)^{*} \aleph^{\mathcal{K}}{ }^{\mathcal{H}} \mathrm{U}_{13}^{\mathcal{K}}\left(\psi(d) \otimes 1_{\mathcal{H}} \otimes 1_{A}\right)\left(\mathrm{U}_{13}^{\mathcal{K}}\right)^{*}\left(\aleph^{\mathcal{K}}\right)_{12}^{*} \\
& =\left(\left(\varphi \otimes \operatorname{id}_{A}\right) \gamma(c)\right)_{13}{ }^{\mathcal{K}} \aleph^{\mathcal{H}}{ }_{12}\left(\left(\psi \otimes \operatorname{id}_{A}\right) \delta(d)\right)_{13}\left(\aleph^{\mathcal{K}}\right)_{12}^{*} \quad \text { for all } c \in C \text { and } d \in D \text {. }
\end{aligned}
$$

The first equality uses Definition 5.8 and 5.20 , the second and third equality use (5.14 and the fourth equality uses the covariance condition 4.13 for $\gamma$ and $\delta$.

Proposition 5.18. $C \bigotimes_{\mathcal{R}} D$ is endowed with a natural (diagonal) coaction $\gamma \bowtie_{\mathcal{R}} \delta: C \bigotimes_{\mathcal{R}} D \rightarrow C \bigotimes_{\mathcal{R}} D \otimes A$ of $\mathbb{G}$ such that

$$
\left(\gamma \bowtie_{\mathcal{R}} \delta\right) \circ \iota_{C}=\left(\iota_{C} \otimes \operatorname{id}_{A}\right) \circ \gamma \quad \text { and } \quad\left(\gamma \bowtie_{\mathcal{R}} \delta\right) \circ \iota_{D}=\left(\iota_{D} \otimes \operatorname{id}_{A}\right) \circ \delta .
$$

Equivalently, $\bigotimes_{\mathcal{R}}: \mathfrak{C}^{*} \mathfrak{a l g}(\mathbb{G}) \times \mathfrak{C}^{*} \mathfrak{a l g}(\mathbb{G}) \rightarrow \mathfrak{C}^{*} \mathfrak{a l g}(\mathbb{G})$ is a covariant functor and $\iota_{C}, \iota_{D}$ are morphisms in the category $\mathfrak{C}^{*} \mathfrak{a l g}(\mathbb{G})$.

Proof. Existence of $\gamma \bowtie_{\mathcal{R}} \delta$ is given by Theorem 5.17 Diagram 2.22 for $\gamma$ and $\delta$ imply:

$$
\left(\operatorname{id}_{C \bigotimes_{\mathcal{R}} D} \otimes \Delta_{A}\right) \circ \gamma \bowtie_{\mathcal{R}} \delta=\left(\left(\gamma \bowtie_{\mathcal{R}} \delta\right) \otimes \operatorname{id}_{A}\right) \circ \gamma \bowtie_{\mathcal{R}} \delta
$$

Now $\gamma$ and $\delta$ satisfy the Podleś condition 2.23). Therefore, Lemma 4.16 and (5.21) yield the Podleś condition 2.23 for $\gamma \bowtie_{\mathcal{R}} \delta$ :

$$
\begin{aligned}
\left(\gamma \bowtie_{\mathcal{R}} \delta\left(C \bigotimes_{\mathcal{R}} D\right)\right) \cdot\left(1_{C \bigotimes_{\mathcal{R}} D} \otimes A\right) & =\left(\iota_{C} \otimes \mathrm{id}_{A}\right) \gamma(C) \cdot\left(\left(\iota_{D} \otimes \operatorname{id}_{A}\right)\left(\delta(D) \cdot\left(1_{D} \otimes A\right)\right)\right. \\
& =\left(\left(\iota_{C} \otimes 1_{A}\right)(\gamma(C)) \cdot\left(\iota_{D}(D) \otimes A\right)\right. \\
& =\left(\iota_{C} \otimes 1_{A}\right)\left(\gamma(C) \cdot\left(1_{C} \otimes A\right)\right) \cdot\left(\iota_{D}(D) \otimes 1_{\mathcal{M}(A)}\right) \\
& =\iota_{C}(C) \cdot \iota_{D}(D) \otimes A=C \otimes_{\mathcal{R}} D \otimes A .
\end{aligned}
$$

Proof of Theorem 5.16, Let $\left(C_{i}, \gamma_{i}\right)$ be $\mathbb{G}$-C $\mathrm{C}^{*}$-algebras and let $\left(\varphi_{i}, \mathrm{U}^{\mathcal{H}_{i}}\right)$ be covariant corepresentations of $\left(C_{i}, \gamma_{i}, A\right)$, respectively, for $i=1,2,3$. By Proposition $5.18\left(C_{2} \bigotimes_{\mathcal{R}} C_{3}, \gamma_{2} \bowtie_{\mathcal{R}} \gamma_{3}\right)$ is a $\mathbb{G}$ - $\mathrm{C}^{*}$-algebra. 
Now $\left(\varphi_{2} \otimes_{\mathcal{R}} \varphi_{3}, \mathrm{U}^{\mathcal{H}_{2}} \oplus \mathrm{U}^{\mathcal{H}_{3}}\right)$ is a covariant representation of $\left(C_{2} \otimes_{\mathcal{R}} C_{3}, \gamma_{2} \bowtie_{\mathcal{R}} \gamma_{3}, A\right)$ on $\mathcal{H} \otimes \mathcal{K}$. Therefore, Theorem 4.27 gives a faithful representation $\varphi_{1} \otimes_{\mathcal{R}}\left(\varphi_{2} \otimes_{\mathcal{R}} \varphi_{3}\right): C_{1} \otimes_{\mathcal{R}}\left(C_{2} \otimes_{\mathcal{R}} C_{3}\right) \rightarrow \mathbb{B}\left(\mathcal{H}_{1} \otimes \mathcal{H}_{2} \otimes \mathcal{H}_{3}\right)$, defined by:

$$
\begin{aligned}
& \left(\varphi_{1} \otimes_{\mathcal{R}}\left(\varphi_{2} \otimes_{\mathcal{R}} \varphi_{3}\right)\right) \circ \iota_{C_{1}}\left(c_{1}\right)=\varphi_{1}\left(c_{1}\right) \otimes 1_{\mathcal{H}_{2}} \otimes 1_{\mathcal{H}_{3}}, \\
& \left(\varphi_{1} \otimes_{\mathcal{R}}\left(\varphi_{2} \otimes_{\mathcal{R}} \varphi_{3}\right)\right) \circ \iota_{C_{2}}\left(c_{2}\right)=\left(\stackrel{\mathcal{H}_{2} \otimes \mathcal{H}_{3} \mathcal{H}_{1}}{\chi}\right)\left(\varphi_{2}\left(c_{2}\right) \otimes 1_{\mathcal{H}_{3}} \otimes 1_{\mathcal{H}_{1}}\right)\left(\stackrel{\mathcal{H}_{2} \otimes \mathcal{H}_{3} \mathcal{H}_{1}}{\chi}\right)^{*}, \\
& \begin{array}{ccc}
\mathcal{H}_{2} \otimes \mathcal{H}_{3} \mathcal{H}_{1} & \mathcal{H}_{3} & \mathcal{H}_{2}
\end{array} \\
& \left(\varphi_{1} \otimes_{\mathcal{R}}\left(\varphi_{2} \otimes_{\mathcal{R}} \varphi_{3}\right)\right) \circ \iota_{C_{3}}\left(c_{3}\right)=(\quad{ })\left(\bigvee_{12}\right)\left(\varphi_{3}\left(c_{3}\right) \otimes 1_{\mathcal{H}_{2}} \otimes 1_{\mathcal{H}_{1}}\right) \\
& \left(\aleph^{\mathcal{H}_{3}} \mathrm{H}^{\mathcal{H}_{2}}\right)^{*}\left(\stackrel{\mathcal{H}_{2} \otimes \mathcal{H}_{3} \mathcal{H}_{1}}{\searrow}\right)^{*}
\end{aligned}
$$

for $c_{i} \in C_{i}, i=1,2,3$.

The diagrams in Proposition 5.9 and Corollary 5.10 give:

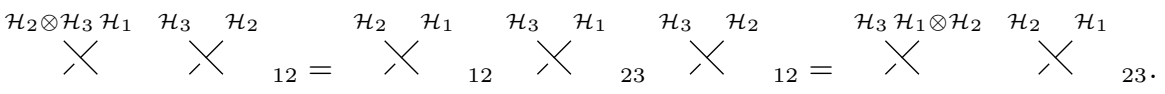

Then the last two equations in 5.22 get reduced to

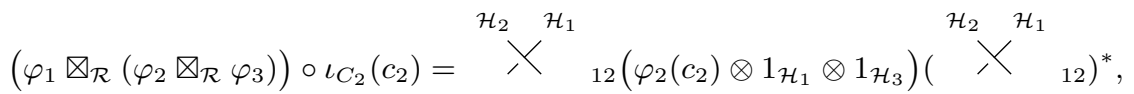

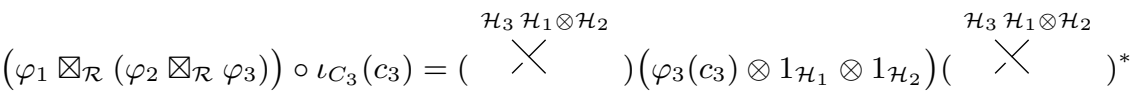

for all $c_{2} \in C_{2}$ and $c_{3} \in C_{3}$.

A similar computation shows that the faithful representation $\left(\varphi_{1} \otimes_{\mathcal{R}} \varphi_{2}\right) \otimes_{\mathcal{R}} \varphi_{3}:\left(C_{1} \otimes_{\mathcal{R}} C_{2}\right) \otimes_{\mathcal{R}} C_{3} \rightarrow$ $\mathbb{B}\left(\mathcal{H}_{1} \otimes \mathcal{H}_{2} \otimes \mathcal{H}_{3}\right)$ acts by:

$$
\begin{aligned}
& \left(\left(\varphi_{1} \otimes_{\mathcal{R}} \varphi_{2}\right) \otimes_{\mathcal{R}} \varphi_{3}\right) \circ \iota_{C_{1}}\left(c_{1}\right)=\varphi_{1}\left(c_{1}\right) \otimes 1_{\mathcal{H}_{2}} \otimes 1_{\mathcal{H}_{3}},
\end{aligned}
$$

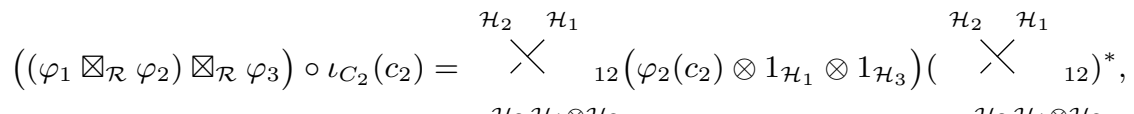

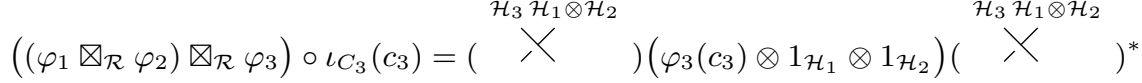

for all $c_{i} \in C_{i}, i=1,2,3$.

Combining (5.23) and (5.24) gives $C_{1} \otimes_{\mathcal{R}}\left(C_{2} \otimes_{\mathcal{R}} C_{3}\right) \cong\left(C_{1} \otimes_{\mathcal{R}} C_{2}\right) \otimes_{\mathcal{R}} C_{3}$.

Remark 5.19. The equations in 5.20 imply

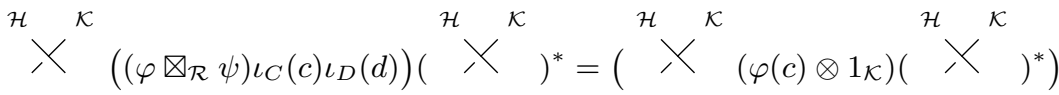

$$
\begin{aligned}
& \left({ }^{\mathcal{H}}{ }^{\mathcal{K}} \aleph^{\mathcal{K}}\left(\psi(d) \otimes 1_{\mathcal{H}}\right)\left(\mathrm{X}^{\mathcal{H}} \mathrm{X}^{\mathcal{K}}\right)^{*}\right)
\end{aligned}
$$

Hence $\aleph^{\mathcal{H}}$ Therefore, by Proposition 5.14 the monoidal category $\mathfrak{C}^{*} \mathfrak{a l g}(\mathbb{G})$ is braided if and only if the $R$-matrix $\mathcal{R} \in \mathcal{U}(\hat{A} \otimes \hat{A})$ is antisymmetric. Then $\mathfrak{C}^{*} \mathfrak{a l g}(\mathbb{G})$ is even symmetric. 


\subsection{Quasitriangularity of the quantum codouble}

The general construction of the $\chi$-Drinfeld double for a given bicharacter $\chi \in \mathcal{U}(\hat{A} \otimes \hat{B})$ has been discussed in Section 4.6. In this section, we restrict our attention to $\widehat{\mathbb{H}}=\mathbb{G}$ and $\chi=\mathrm{W} \in \mathcal{U}(\hat{A} \otimes A)$.

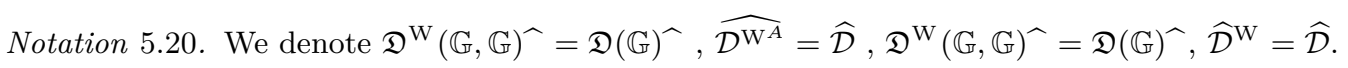

Definition 5.21. The pairs $\mathfrak{D}(\mathbb{G})=\left(\mathcal{D}, \Delta_{\mathcal{D}}\right)$ and $\mathfrak{D}(\mathbb{G})^{\wedge}=\left(\widehat{\mathcal{D}}, \Delta_{\widehat{\mathcal{D}}}\right)$ are called the Drinfeld double and the quantum codouble of $\mathbb{G}$.

Let $(\pi, \hat{\pi})$ be a $\mathbb{G}$-Heisenberg pair acting on $\mathcal{H}$. Then the corresponding representations of $A$ and $\hat{A}$ defined in 4.20 reduce to

$$
\begin{array}{ll}
\rho(a):=\left((\bar{\pi} \otimes \pi) \Delta_{A}(a)\right)_{13} & \text { in } \mathbb{B}\left(\mathcal{H}_{\mathcal{D}}\right) \text { for all } a \in A, \\
\theta(\hat{a}):=\left(\left(\overline{\hat{\pi}} \otimes(\hat{\pi} \otimes \hat{\pi}) \hat{\Delta}_{A}\right) \hat{\Delta}_{A}(\hat{a})\right) & \text { in } \mathbb{B}\left(\mathcal{H}_{\mathcal{D}}\right) \text { for all } \hat{a} \in \hat{A}, \\
\xi(\hat{a}):=1_{\overline{\mathcal{H}}} \otimes 1_{\mathcal{H}} \otimes \hat{\pi}(\hat{a}) & \text { in } \mathbb{B}\left(\mathcal{H}_{\mathcal{D}}\right) \text { for all } \hat{a} \in \hat{A}, \\
\zeta(a):=1_{\overline{\mathcal{H}}} \otimes \pi(a) \otimes 1_{\mathcal{H}} & \text { in } \mathbb{B}\left(\mathcal{H}_{\mathcal{D}}\right) \text { for all } a \in A .
\end{array}
$$

As before, $(\bar{\pi}, \overline{\hat{\pi}})$ is the $\mathbb{G}$-anti-Heisenberg pair on $\overline{\mathcal{H}}$ corresponding to $(\pi, \hat{\pi})$ by Example 2.19 . Therefore, the Hilbert space $\mathcal{H}_{\mathcal{D}}=\overline{\mathcal{H}} \otimes \mathcal{H} \otimes \mathcal{H}$ has one leg less than the general construction in Section 4.6 .

$\mathbb{W}^{\mathcal{D}}=\mathrm{W}_{\theta \zeta} \widehat{\mathrm{W}}_{\rho \xi} \in \mathcal{U}\left(\mathcal{H}_{\mathcal{D}} \otimes \mathcal{H}_{\mathcal{D}}\right)$ is a multiplicative unitary for the quantum codouble $\mathfrak{D}(\mathbb{G})^{\Upsilon}$, and $\mathfrak{D}(\mathbb{G})$ is dual to it. Selecting $\chi=\mathrm{W}$ in Definition 4.52 gives $\mathrm{W}$-Yetter-Drinfeld $\mathrm{C}^{*}$-algebras.

Terminology 5.22. We use the term $\mathbb{G}$-Yetter-Drinfeld as a synonym for W-Yetter-Drinfeld.

In particular, a $C^{*}$-algebra $C$ is $\mathbb{G}$-Yetter-Drinfeld for a pair of coactions $(\gamma, \delta)$ of $\mathbb{G}$ and $\widehat{\mathbb{G}}$ on $C$ if and only if

$$
\sigma_{23}^{\mathrm{W}}\left(\left(\gamma \otimes \mathrm{id}_{\hat{A}}\right) \delta\right)=\left(\delta \otimes \mathrm{id}_{A}\right) \gamma
$$

Notation 5.23. Let $\mathcal{Y} \mathcal{D} \mathfrak{C}^{*} \mathfrak{a l g}(\mathbb{G})$ be the category with $\mathbb{G}$-Yetter-Drinfeld $\mathrm{C}^{*}$-algebras as objects, and $\mathbb{G}$ and $\widehat{\mathbb{G}}$-equivariant morphisms as arrows.

Let $\mathbb{G}$ be a quasitriangular quantum group with $R$-matrix $\mathcal{R} \in \mathcal{U}(\hat{A} \otimes \hat{A})$. Let $\Delta_{R}: A \rightarrow A \otimes \hat{A}$ be the associated right quantum group homomorphism. Theorem 3.27 induces a dual coaction $\delta: C \rightarrow C \otimes \hat{A}$ for any $\mathbb{G}-\mathrm{C}^{*}$-algebra $(C, \gamma)$.

Lemma 5.24. The pair $(\gamma, \delta)$ as above is a $\mathbb{G}$-Yetter-Drinfeld pair.

Proof. By Lemma 2.34 any object $C \in \mathfrak{C}^{*} \mathfrak{a l g}(\mathbb{G})$ is equivariantly isomorphic to a sub-object of $D \otimes A$, for some $C^{*}$-algebra $D$, with trivial coaction on $D$. Therefore, it suffices to prove the statement for $C=A$ and $\gamma=\Delta_{A}$. Then $\delta=\Delta_{R}$ by (3.25) and 2.22). Using the relation (5.7) for $\mathcal{R} \in \mathcal{U}(\hat{A} \otimes \hat{A})$, up to correct modification for dual multiplicative unitaries, we get:

$$
\mathcal{R}_{12} \mathrm{~W}_{13} \mathrm{~W}_{23}=\mathrm{W}_{23} \mathrm{~W}_{13} \mathcal{R}_{12} \quad \text { in } \mathcal{U}(\hat{A} \otimes \hat{A} \otimes A) .
$$

Then using the last equation, 2.8 and 3.15 we compute

$$
\sigma_{34}^{\mathrm{W}}\left(\left(\mathrm{id}_{\hat{A}} \otimes\left(\Delta_{A} \otimes \mathrm{id}_{\hat{A}}\right) \Delta_{R}\right) \mathrm{W}\right)=\mathrm{W}_{12} \sigma_{34}^{\mathrm{W}}\left(\mathrm{W}_{13} \mathcal{R}_{14}\right)=\mathrm{W}_{12} \mathcal{R}_{13} \mathrm{~W}_{14}=\left(\mathrm{id}_{\hat{A}} \otimes\left(\Delta_{R} \otimes \mathrm{id}_{A}\right) \Delta_{A}\right) \mathrm{W} .
$$

Finally, slicing the first leg of the last computation by $\omega \in \hat{A}^{\prime}$ gives 5.26$)$ for the pair $\left(\Delta_{A}, \Delta_{R}\right)$, which completes the proof. 


\subsubsection{R-matrix on the Drinfeld double}

Proposition 5.25. The quantum codouble $\mathfrak{D}(\mathbb{G})^{\uparrow}$ is a quasitriangular quantum group with $R$-matrix $\mathrm{W}_{\theta \rho} \in \mathcal{U}(\mathcal{D} \otimes \mathcal{D})$.

Proof. Since $\rho \in \operatorname{Mor}(A, \mathcal{D})$ and $\theta \in \operatorname{Mor}(\hat{A}, \mathcal{D})$ are Hopf ${ }^{*}$-homomorphisms, $\mathrm{W}_{\theta \rho} \in \mathcal{U}(\mathcal{D} \otimes \mathcal{D})$ is a bicharacter. We only need to show (5.1) for $\mathrm{W}_{\theta \rho}$. Lemma 4.452 implies:

$$
\begin{aligned}
\mathrm{W}_{\theta_{2} \rho_{3}}\left(\left(\mathrm{id}_{\hat{A}} \otimes(\rho \otimes \rho) \sigma \circ \Delta_{A}\right) \mathrm{W}\right) \mathrm{W}_{\theta_{2} \rho_{3}}^{*} & =\mathrm{W}_{\theta_{2} \rho_{3}} \mathrm{~W}_{1 \rho_{3}} \mathrm{~W}_{1 \rho_{2}} \mathrm{~W}_{\theta_{2} \rho_{3}}^{*} \\
& =\mathrm{W}_{1 \rho_{2}} \mathrm{~W}_{1 \rho_{3}}=\left(\operatorname{id}_{\hat{A}} \otimes(\rho \otimes \rho) \circ \Delta_{A}\right) \mathrm{W},
\end{aligned}
$$

where $\rho_{i}$ denotes $\rho$ acting on the $i$ th leg. Taking slices on the first leg by linear functionals $\omega \in \hat{A}^{\prime}$ gives

$$
\mathrm{W}_{\theta \rho}\left((\rho \otimes \rho) \sigma \circ \Delta_{A}(a)\right) \mathrm{W}_{\theta \rho}^{*}=(\rho \otimes \rho) \Delta_{A}(a) \quad \text { for all } a \in A .
$$

Similarly, $\mathrm{W}_{\theta \rho}\left((\theta \otimes \theta) \sigma \circ \hat{\Delta}_{A}(\hat{a})\right) \mathrm{W}_{\theta \rho}^{*}=(\theta \otimes \theta) \hat{\Delta}_{A}(\hat{a})$ for all $\hat{a} \in \hat{A}$. Combining the last two identities gives 5.1 for $\mathrm{W}_{\theta \rho}$ :

$$
\mathrm{W}_{\theta \rho}\left(\sigma \Delta_{\mathcal{D}}(\theta(\hat{a}) \rho(a))\right) \mathrm{W}_{\theta \rho}^{*}=\Delta_{\mathcal{D}}(\theta(\hat{a}) \rho(a)) \quad \text { for all } a \in A, \hat{a} \in \hat{A} .
$$

From now on we fix the $R$-matrix $\mathcal{R}=\mathrm{W}_{\theta \rho} \in \mathcal{U}(\mathcal{D} \otimes \mathcal{D})$.

The quasitriangularity of $\mathfrak{D}(\mathbb{G})^{\wedge}$ provides a braided monoidal structure on $\operatorname{Corep}(\mathfrak{D}(\mathbb{G}) \widehat{)}$, and the monoidal structure on $\mathfrak{C}^{*} \mathfrak{a l g}\left(\mathfrak{D}(\mathbb{G})^{\wedge}\right)$ gets induced from it. Therefore, we discuss the corepresentation theory of $\mathfrak{D}(\mathbb{G})^{\wedge}$ first. Let $\mathrm{U} \in \mathcal{U}(\mathbb{K}(\mathcal{K}) \otimes A)$ and $\mathrm{V} \in \mathcal{U}(\mathbb{K}(\mathcal{K}) \otimes \hat{A})$ be corepresentations of $\mathbb{G}$ and $\widehat{\mathbb{G}}$ on a Hilbert space $\mathcal{K}$.

Definition 5.26. A pair $(\mathrm{U}, \mathrm{V})$ is called $\mathfrak{D}(\mathbb{G})^{\widehat{ }}$-compatible if they commute in the following way:

$$
\sigma_{23}^{\mathrm{W}}\left(\mathrm{U}_{12} \mathrm{~V}_{13}\right)=\mathrm{V}_{12} \mathrm{U}_{13} \quad \text { in } \mathcal{U}(\mathbb{K}(\mathcal{K}) \otimes \hat{A} \otimes A) .
$$

Lemma 5.27. A pair $(\mathrm{U}, \mathrm{V})$ is $\mathfrak{D}(\mathbb{G})^{-}$-compatible if and only if $(\mathrm{V}, \mathrm{U})$ is $\mathfrak{D}(\widehat{\mathbb{G}})^{-}$-compatible.

Example 5.28. As a special case of Lemma 4.53 the pair of corepresentations $\left(\mathrm{W}_{\theta 2}, \widehat{\mathrm{W}}_{\rho 2}\right)$ of $\mathbb{G}$ and $\widehat{\mathbb{G}}$ on $\mathcal{H}_{\mathcal{D}}$

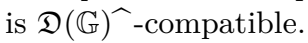

Another class of examples of $\mathfrak{D}(\mathbb{G})^{\uparrow}$-compatible pairs of corepresentations come from the corepresentations of quasitriangular quantum groups.

Example 5.29. Let $\mathbb{G}$ be a quasitriangular quantum group with $R$-matrix $\mathcal{R} \in \mathcal{U}(\hat{A} \otimes \hat{A})$ and induced right quantum group homomorphism $\Delta_{R}: A \rightarrow A \otimes \hat{A}$. By Proposition 3.31 given any corepresentation $\mathrm{U} \in \mathcal{U}(\mathbb{K}(\mathcal{H}) \otimes A)$ of $\mathbb{G}$ there is a unique corepresentation $\mathrm{V} \in \mathcal{U}(\mathbb{K}(\mathcal{H}) \otimes \hat{A})$ of $\widehat{\mathbb{G}}$ satisfying (3.27):

$$
\left(\operatorname{id}_{\mathcal{H}} \otimes \Delta_{R}\right) \mathrm{U}=\mathrm{U}_{12} \mathrm{~V}_{13} \quad \text { in } \mathcal{U}(\mathbb{K}(\mathcal{H}) \otimes A \otimes \hat{A}) .
$$

The proof of Lemma 5.24 shows that $\left(\Delta_{A}, \Delta_{R}\right)$ is a $\mathbb{G}$-Yetter-Drinfeld pair. Equations 2.25) and (5.28) yield

$$
\mathrm{U}_{12} \sigma_{34}^{\mathrm{W}}\left(\mathrm{U}_{13} \mathrm{~V}_{14}\right)=\sigma_{34}^{\mathrm{W}}\left(\left(\mathrm{id}_{\mathcal{H}} \otimes\left(\Delta_{A} \otimes \mathrm{id}_{\hat{A}}\right) \Delta_{R}\right) \mathrm{U}\right)=\left(\mathrm{id}_{\mathcal{H}} \otimes\left(\Delta_{R} \otimes \mathrm{id}_{A}\right) \Delta_{A}\right) \mathrm{U}=\mathrm{U}_{12} \mathrm{~V}_{13} \mathrm{U}_{14}
$$

Cancelling $\mathrm{U}_{12}$ on both sides of the last equation yields 5.27 for (U, V).

In general, any corepresentation can be viewed as a cocycle for the trivial coaction (see Example 4.39). In particular, Proposition 4.55 establishes a bijective correspondence between corepresentations of $\mathfrak{D}(\mathbb{G})^{\wedge}$ satisfying 4.18 and $\mathfrak{D}(\mathbb{G})^{\widehat{\wedge}}$-compatible pairs, each of them satisfying 4.18. This is true for all $\mathfrak{D}(\mathbb{G})^{\wedge}$-corepresentations.

Proposition 5.30. Every $\mathfrak{D}(\mathbb{G})^{\uparrow}$-compatible pair of corepresentations $\mathrm{U} \in \mathcal{U}(\mathbb{K}(\mathcal{K}) \otimes A)$ and $\mathrm{V} \in \mathcal{U}(\mathbb{K}(\mathcal{K}) \otimes$ $\hat{A})$ gives rise to a corepresentation $\mathrm{X} \in \mathcal{U}(\mathbb{K}(\mathcal{K}) \otimes \widehat{\mathcal{D}})$ of $\mathfrak{D}(\mathbb{G})^{\curlywedge}$ on $\mathcal{K}$, defined by

$$
\mathrm{X}:=\mathrm{U}_{12} \mathrm{~V}_{13} \quad \text { in } \mathcal{U}(\mathbb{K}(\mathcal{K}) \otimes A \otimes \hat{A}) .
$$

Conversely, every corepresentation of $\mathfrak{D}(\mathbb{G})^{\wedge}$ comes from a unique $\mathfrak{D}(\mathbb{G})^{\uparrow}$-compatible pair of corepresentations acting on the same Hilbert space. 
Proof. Let $(\mathrm{U}, \mathrm{V})$ be a $\mathfrak{D}(\mathbb{G})^{\widehat{ }}$-compatible pair of corepresentations on $\mathcal{H}$. The following computation yields that $\mathrm{X}$ defined in 5.29 is a corepresentation of $\mathfrak{D}(\mathbb{G})^{\wedge}$ :

$$
\begin{aligned}
\left(\operatorname{id}_{\mathcal{K}} \otimes \hat{\Delta}_{\mathcal{D}}\right) \mathrm{X}=\sigma_{34}^{\mathrm{W}}\left(\left(\operatorname{id} \mathcal{K} \otimes \Delta_{A} \otimes \hat{\Delta}_{A}\right) \mathrm{U}_{12} \mathrm{~V}_{13}\right)=\mathrm{U}_{12}\left(\sigma_{34}^{\mathrm{W}}\left(\mathrm{U}_{13} \mathrm{~V}_{14}\right)\right) \mathrm{V}_{15}=\mathrm{U}_{12} \mathrm{~V}_{13} \mathrm{U}_{14} \mathrm{~V}_{15} & \\
& =\mathrm{X}_{123} \mathrm{X}_{145}
\end{aligned}
$$

Conversely, let $\mathrm{X} \in \mathcal{U}(\mathbb{K}(\mathcal{K}) \otimes \widehat{\mathcal{D}})$ be a corepresentation of $\mathfrak{D}(\mathbb{G})^{\wedge}$ on $\mathcal{K}$. Following Example 4.54 we obtain the right quantum group homomorphisms $\Delta_{R}=\mathrm{id}_{A} \otimes \hat{\Delta}_{A}$ and $\Delta_{R}^{\prime}=\sigma_{23}^{\mathrm{W}}\left(\Delta_{A} \otimes \mathrm{id}_{\hat{A}}\right)$ associated to the bicharacters $\widehat{\mathrm{W}}_{\rho 2} \in \mathcal{U}(\mathcal{D} \otimes \hat{A})$ and $\mathrm{W}_{\theta 2} \in \mathcal{U}(\mathcal{D} \otimes A)$, respectively. Moreover, $\widehat{\mathcal{D}}$ becomes a $\mathbb{G}$-Yetter-Drinfeld $\mathrm{C}^{*}$-algebra with respect to these coactions; hence $\Delta_{R}$ and $\Delta_{R}^{\prime}$ satisfy (5.26). Equivalently, we have the following compatibility condition:

$$
\left.\sigma_{23}^{\mathrm{W}}\left(\left(\Delta_{R}^{\prime} \otimes \operatorname{id}_{\hat{A}}\right) \Delta_{R}\right)\right)=\left(\Delta_{R} \otimes \operatorname{id}_{A}\right) \Delta_{R}^{\prime}
$$

By Proposition 3.31 and 3.27 we get corepresentations $\mathrm{U} \in \mathcal{U}(\mathbb{K}(\mathcal{K}) \otimes A)$ of $\mathbb{G}$ and $\mathrm{V} \in \mathcal{U}(\mathbb{K}(\mathcal{K}) \otimes \hat{A})$ of $\widehat{\mathbb{G}}$ on $\mathcal{K}$ defined by:

$$
\begin{array}{ll}
\left(\operatorname{id}_{\mathcal{K}} \otimes \Delta_{R}^{\prime}\right) \mathrm{X}=\mathrm{X}_{12} \mathrm{U}_{13} & \text { in } \mathcal{U}(\mathbb{K}(\mathcal{K}) \otimes \widehat{D} \otimes A), \\
\left(\operatorname{id}_{\mathcal{K}} \otimes \Delta_{R}\right) \mathrm{X}=\mathrm{X}_{12} \mathrm{~V}_{13} & \text { in } \mathcal{U}(\mathbb{K}(\mathcal{K}) \otimes \widehat{D} \otimes \hat{A}) .
\end{array}
$$

The compatibility condition 5.30 between $\Delta_{R}$ and $\Delta_{R}^{\prime}$ and then 5.31 yield:

$$
\left(\operatorname{id}_{\mathcal{K}} \otimes\left(\Delta_{R} \otimes \mathrm{id}_{A}\right) \Delta_{R}^{\prime}\right) \mathrm{X}=\sigma_{34}^{\mathrm{W}}\left(\mathrm{id}_{\mathcal{K}} \otimes\left(\Delta_{R}^{\prime} \otimes \mathrm{id}_{\hat{A}}\right) \Delta_{R}\right) \mathrm{X}=\mathrm{X}_{12} \sigma_{34}^{\mathrm{W}}\left(\mathrm{U}_{13} \mathrm{~V}_{14}\right)
$$

Immediate use of 5.31 gives:

$$
\left(\operatorname{id}_{\mathcal{K}} \otimes\left(\Delta_{R} \otimes \operatorname{id}_{A}\right) \Delta_{R}^{\prime}\right) \mathrm{X}=\mathrm{X}_{12} \mathrm{~V}_{13} \mathrm{U}_{14}
$$

Combining the right hand sides of the last two equations and cancelling the unitary $\mathrm{X}_{12}$ establishes 5.27 for $(\mathrm{U}, \mathrm{V})$.

\subsubsection{Coaction category of the quantum codouble}

Let $\left(C_{i}, \lambda_{i}\right)$ be $\mathfrak{D}(\mathbb{G})^{-}-\mathrm{C}^{*}$-algebras and let $\left(\mathrm{X}^{\mathcal{K}_{i}}, \varphi_{i}\right)$ be faithful covariant representations of $\left(C_{i}, \tilde{\gamma}_{i}, \widehat{\mathcal{D}}\right)$ on Hilbert spaces $\mathcal{K}_{i}$ for $i=1,2$. By virtue of Proposition 4.55 for $\mathfrak{D}(\mathbb{G})^{\wedge}$ and Proposition 5.30 we can always decompose $\left(\mathrm{X}^{\mathcal{K}_{i}}, \varphi_{i}\right)$ into two parts: $\left(\mathrm{U}^{\mathcal{K}_{i}}, \varphi_{i}\right)$, a faithful covariant representations of $\left(C_{i}, \gamma_{i}, A\right)$ on $\mathcal{H}_{i}$, and $\left(\mathrm{V}^{\mathcal{K}_{i}}, \varphi_{i}\right)$, a faithful covariant representations of $\left(C_{i}, \delta_{i}, \hat{A}\right)$ on $\mathcal{K}_{i}$ for $i=1,2$, respectively. Definition 4.21 gives the following conditions:

$$
\begin{array}{ll}
\left(\varphi_{i} \otimes \operatorname{id}_{A}\right) \gamma_{i}\left(c_{i}\right)=\left(\mathrm{U}^{\mathcal{K}_{i}}\right)\left(\varphi_{i}\left(c_{i}\right) \otimes 1_{A}\right)\left(\mathrm{U}^{\mathcal{K}_{i}}\right)^{*} & \text { in } \mathcal{U}\left(\mathbb{K}\left(\mathcal{K}_{i}\right) \otimes A\right), \\
\left(\varphi_{i} \otimes \operatorname{id}_{\hat{A}}\right) \delta_{i}\left(c_{i}\right)=\left(\mathrm{V}^{\mathcal{K}_{i}}\right)\left(\varphi_{i}\left(c_{i}\right) \otimes 1_{\hat{A}}\right)\left(\mathrm{V}^{\mathcal{K}_{i}}\right)^{*} & \text { in } \mathcal{U}\left(\mathbb{K}\left(\mathcal{K}_{i}\right) \otimes \hat{A}\right),
\end{array}
$$

for $i=1,2$.

Then $\gamma_{i}: C_{i} \rightarrow C_{i} \otimes A$ and $\delta_{i}: C_{i} \rightarrow C_{i} \otimes \hat{A}$ satisfy the $\mathbb{G}$-Yetter-Drinfeld compatibility condition 5.26 for $i=1$, 2. Similarly, the corepresentations $\mathrm{U}^{\mathcal{K}_{i}} \in \mathcal{U} \mathcal{M}\left(\mathbb{K}\left(\mathcal{K}_{i}\right) \otimes A\right)$ and $\mathrm{V}^{\mathcal{K}_{i}} \in \mathcal{U}(\mathbb{K}(\mathcal{K}) \otimes \hat{A})$ satisfy 5.27 for $i=1,2$.

We already know, from the proof of Proposition 5.30 that $\left(\operatorname{id}_{\widehat{\mathcal{D}}} \otimes \rho\right) \Delta_{R}^{\prime}=\left(\operatorname{id}_{\widehat{\mathcal{D}}} \otimes \rho\right)\left(\sigma_{23}^{\mathrm{W}}\left(\Delta_{A} \otimes \mathrm{id}_{\hat{A}}\right)\right)$ is the right quantum group homomorphism associated to the $R$-matrix $\mathcal{R}=\mathrm{W}_{\theta \rho}$ (recall $\theta$ and $\rho$ from $(5.25)$ ). The corresponding $\mathcal{R}$-Heisenberg pair $(\alpha, \beta)$ defined in Lemma 4.11 becomes

$$
\alpha(a \otimes \hat{a}):=(\zeta * \xi \otimes \rho)\left(\sigma_{23}^{\mathrm{W}}\left(\Delta_{A}(a) \otimes \hat{a}\right)\right) \quad \text { and } \quad \beta(a \otimes \hat{a}):=1_{\mathcal{H}_{\mathcal{D}}} \otimes \zeta(a) \xi(\hat{a}),
$$

where we denote $\zeta * \xi(a \otimes \hat{a}):=\zeta(a) \xi(\hat{a})$ for $a \in A$ and $\hat{a} \in \hat{A}$.

The following lemma characterises the braiding induced by the pair $\left(\mathrm{X}^{\mathcal{K}_{1}}, \mathrm{X}^{\mathcal{K}_{2}}\right)$ of corepresentations of $\mathfrak{D}(\mathbb{G})^{\wedge}$ in terms of the pair $\left(\mathrm{U}^{\mathcal{K}_{1}}, \mathrm{~V}^{\mathcal{K}_{2}}\right)$. 
Lemma 5.31. Assume the unitary $Z \in \mathcal{U}\left(\mathcal{K}_{1} \otimes \mathcal{K}_{2}\right)$ satisfies 2.30 for the pair of corepresentations $\mathrm{U}^{\mathcal{K}_{1}} \in \mathcal{U}\left(\mathbb{K}\left(\mathcal{K}_{1}\right) \otimes A\right)$ and $\mathrm{V}^{\mathcal{K}_{2}} \in \mathcal{U}\left(\mathbb{K}\left(\mathcal{K}_{2}\right) \otimes \hat{A}\right)$. Then $Z \in \mathcal{U}\left(\mathcal{K}_{1} \otimes \mathcal{K}_{2}\right)$ is the unique solution of the following equation:

$$
\mathrm{X}_{1 \alpha}^{\mathcal{K}_{1}} \mathrm{X}_{2 \beta}^{\mathcal{K}_{2}} Z_{12}=\mathrm{X}_{2 \beta}^{\mathcal{K}_{2}} \mathrm{X}_{1 \alpha}^{\mathcal{K}_{1}} \quad \text { in } \mathcal{U}\left(\mathbb{K}\left(\mathcal{K}_{1} \otimes \mathcal{K}_{2}\right) \otimes \widehat{\mathcal{D}}\right)
$$

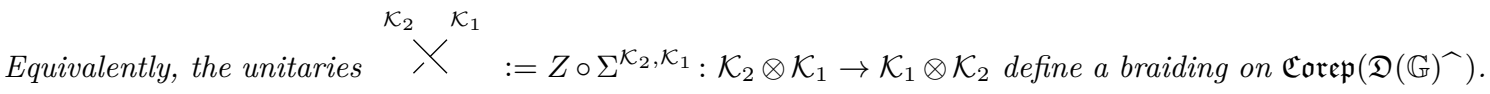

Proof. The solution of (5.34 is clearly unique if it exists. Hence we only need to check that $Z \in \mathcal{U}\left(\mathcal{K}_{1} \otimes \mathcal{K}_{2}\right)$ in 2.30 satisfies 5.34. The pair $\left(\mathrm{U}^{\mathcal{K}_{1}}, \mathrm{~V}^{\mathcal{K}_{1}}\right)$ is $\mathfrak{D}(\mathbb{G})^{-}$-compatible. Therefore, with the assignment of legs $1,2,3$ to $\mathcal{K}_{1}, \mathcal{H}_{\mathcal{D}}, \mathcal{H}_{\mathcal{D}}$ we get

$$
\mathrm{X}_{1 \alpha}^{\mathcal{K}_{1}}=\mathrm{U}_{1 \zeta_{2}}^{\mathcal{K}_{1}}\left(\sigma_{34}^{\mathrm{W}}\left(\mathrm{U}_{13}^{\mathcal{K}_{1}} \mathrm{~V}_{14}^{\mathcal{K}_{1}}\right)\right)_{1(\zeta * \xi) \rho_{3}}=\mathrm{U}_{1 \zeta_{2}}^{\mathcal{K}_{1}} \mathrm{~V}_{1 \xi_{2}}^{\mathcal{K}_{1}} \mathrm{U}_{1 \rho_{3}}^{\mathcal{K}_{1}}
$$

where the notation $\zeta_{i}$ means that $\zeta$ is acting on the $i$ th leg.

Next we assign the legs $1,2,3,4$ to $\mathcal{K}_{1}, \mathcal{K}_{2}, \mathcal{H}_{\mathcal{D}}, \mathcal{H}_{\mathcal{D}}$ and compute:

$$
\mathrm{X}_{1 \alpha}^{\mathcal{K}_{1}} \mathrm{X}_{2 \beta}^{\mathcal{K}_{2}} Z_{12}=\mathrm{U}_{1 \zeta_{3}}^{\mathcal{K}_{1}} \mathrm{~V}_{1 \xi_{3}}^{\mathcal{K}_{1}} \mathrm{U}_{1 \rho_{4}}^{\mathcal{K}_{1}} \mathrm{U}_{2 \zeta_{4}}^{\mathcal{K}_{2}} \mathrm{~V}_{2 \xi_{4}}^{\mathcal{K}_{2}} Z_{12}=\mathrm{U}_{1 \zeta_{3}}^{\mathcal{K}_{1}} \mathrm{~V}_{1 \xi_{3}}^{\mathcal{K}_{1}} \mathrm{U}_{2 \zeta_{4}}^{\mathcal{K}_{2}} \mathrm{U}_{1 \rho_{4}}^{\mathcal{K}_{1}} \mathrm{~V}_{2 \xi_{4}}^{\mathcal{K}_{2}} Z_{12},
$$

where the second equality uses that $\rho$ and $\zeta$ commute on the fourth leg.

Lemma 4.45 1 shows that $(\rho, \xi)$ is a $\mathbb{G}$-Heisenberg pair. Therefore, first using 2.30 and the trivial commutation of unitaries acting on legs 13 and 24 gives:

$$
\mathrm{X}_{1 \alpha}^{\mathcal{K}_{1}} \mathrm{X}_{2 \beta}^{\mathcal{K}_{2}} Z_{12}=\mathrm{U}_{1 \zeta_{3}}^{\mathcal{K}_{1}} \mathrm{~V}_{1 \xi_{3}}^{\mathcal{K}_{1}} \mathrm{U}_{2 \zeta_{4}}^{\mathcal{K}_{2}} \mathrm{~V}_{2 \xi_{4}}^{\mathcal{K}_{2}} \mathrm{U}_{1 \rho_{4}}^{\mathcal{K}_{1}}=\mathrm{U}_{2 \zeta_{4}}^{\mathcal{K}_{2}} \mathrm{~V}_{2 \xi_{4}}^{\mathcal{K}_{2}} \mathrm{U}_{1 \zeta_{3}}^{\mathcal{K}_{1}} \mathrm{~V}_{1 \xi_{3}}^{\mathcal{K}_{1}} \mathrm{U}_{1 \rho_{4}}^{\mathcal{K}_{1}}=\mathrm{X}_{2 \beta}^{\mathcal{K}_{2}} \mathrm{X}_{1 \alpha}^{\mathcal{K}_{1}}
$$

Corollary 5.32. Let $Z \in \mathcal{U}\left(\mathcal{K}_{1} \otimes \mathcal{K}_{2}\right)$ be as in Lemma 5.31. Then

$$
\begin{array}{ll}
Z_{12} \mathrm{U}_{23}^{\mathcal{K}_{2}} \mathrm{U}_{13}^{\mathcal{K}_{1}}=\mathrm{U}_{13}^{\mathcal{K}_{1}} \mathrm{U}_{23}^{\mathcal{K}_{2}} Z_{12} & \text { in } \mathcal{U}\left(\mathbb{K}\left(\mathcal{K}_{1} \otimes \mathcal{K}_{2}\right) \otimes A\right), \\
Z_{12} \mathrm{~V}_{23}^{\mathcal{K}_{2}} \mathrm{~V}_{13}^{\mathcal{K}_{1}}=\mathrm{V}_{13}^{\mathcal{K}_{1}} \mathrm{~V}_{23}^{\mathcal{K}_{2}} Z_{12} & \text { in } \mathcal{U}\left(\mathbb{K}\left(\mathcal{K}_{1} \otimes \mathcal{K}_{2}\right) \otimes \hat{A}\right) .
\end{array}
$$

Proof. The braiding $\nearrow^{\mathcal{K}_{2}} \chi^{\mathcal{K}_{1}}$ on $\operatorname{Corep}\left(\mathfrak{D}(\mathbb{G})^{\wedge}\right)$ intertwines $\mathrm{X}^{\mathcal{K}_{2}} \oplus \mathrm{X}^{\mathcal{K}_{1}}$ and $\mathrm{X}^{\mathcal{K}_{1}} \oplus \mathrm{X}^{\mathcal{K}_{2}}$ or, equivalently, it satisfies

$$
Z_{12} \mathrm{X}_{23}^{\mathcal{K}_{2}} \mathrm{X}_{13}^{\mathcal{K}_{1}}=\mathrm{X}_{13}^{\mathcal{K}_{1}} \mathrm{X}_{23}^{\mathcal{K}_{2}} Z_{12} \quad \text { in } \mathcal{U}\left(\mathbb{K}\left(\mathcal{K}_{1} \otimes \mathcal{K}_{2}\right) \otimes A\right)
$$

Define the right quantum group homomorphism $\Delta_{R}^{\prime}: \widehat{\mathcal{D}} \rightarrow \widehat{\mathcal{D}} \otimes A$ associated to the bicharacter $\mathrm{W}_{\theta 2} \in$ $\mathcal{U}(\mathcal{D} \otimes A)$ as in Example 4.54 Applying id $\mathcal{K}_{1} \otimes \mathcal{K}_{2} \otimes \Delta_{R}^{\prime}$ on the left hand side of 5.36 and using the first relation from (5.31) yields:

$$
\left(\operatorname{id}_{\mathcal{K}_{1} \otimes \mathcal{K}_{2}} \otimes \Delta_{R}^{\prime}\right)\left(Z_{12} \mathrm{X}_{23}^{\mathcal{K}_{2}} \mathrm{X}_{13}^{\mathcal{K}_{1}}\right)=Z_{12} \mathrm{X}_{23}^{\mathcal{K}_{2}} \mathrm{U}_{24}^{\mathcal{K}_{2}} \mathrm{X}_{13}^{\mathcal{K}_{1}} \mathrm{U}_{14}^{\mathcal{K}_{2}}=Z_{12} \mathrm{X}_{23}^{\mathcal{K}_{2}} \mathrm{X}_{13}^{\mathcal{K}_{1}} \mathrm{U}_{24}^{\mathcal{K}_{2}} \mathrm{U}_{14}^{\mathcal{K}_{2}}=\mathrm{X}_{13}^{\mathcal{K}_{1}} \mathrm{X}_{23}^{\mathcal{K}_{2}} Z_{12} \mathrm{U}_{24}^{\mathcal{K}_{2}} \mathrm{U}_{14}^{\mathcal{K}_{2}} .
$$

Similar computations for the right hand side of 5.36 yield:

$$
\left(\operatorname{id}_{\mathcal{K}_{1} \otimes \mathcal{K}_{2}} \otimes \Delta_{R}^{\prime}\right)\left(\mathrm{X}_{13}^{\mathcal{K}_{1}} \mathrm{X}_{23}^{\mathcal{K}_{2}} Z_{12}\right)=\mathrm{X}_{13}^{\mathcal{K}_{1}} \mathrm{U}_{14}^{\mathcal{K}_{2}} \mathrm{X}_{23}^{\mathcal{K}_{2}} \mathrm{U}_{24}^{\mathcal{K}_{2}} Z_{12}=\mathrm{X}_{13}^{\mathcal{K}_{1}} \mathrm{X}_{23}^{\mathcal{K}_{2}} \mathrm{U}_{14}^{\mathcal{K}_{2}} \mathrm{U}_{24}^{\mathcal{K}_{2}} Z_{12}
$$

Cancellation of the unitary $\mathrm{X}_{13}^{\mathcal{K}_{1}} \mathrm{X}_{23}^{\mathcal{K}_{2}}$ from the right hand side of the last two expressions yields the first relation in 5.35.

Using the right quantum homomorphism $\Delta_{R}: \widehat{\mathcal{D}} \rightarrow \widehat{\mathcal{D}} \otimes \hat{A}$ associated to the bicharacter $\widehat{\mathrm{W}}_{\rho 2} \in \mathcal{U}(\mathcal{D} \otimes \hat{A})$ in a similar manner, we obtain the second relation in (5.35).

Corollary 5.33. The braiding $\chi^{\mathcal{K}_{2}}:=Z \circ \Sigma^{\mathcal{K}_{1}}, \mathcal{K}_{1}$ in Lemma 5.31 is symmetric if and only if $\mathrm{W}=\widehat{\mathrm{W}}$. 
Proof. By the universal property 2.34) of $\tilde{\mathcal{V}} \in \mathcal{U}\left(\hat{A}^{\mathrm{u}} \otimes A\right)$ there is a unique morphism $\varphi: \hat{A}^{\mathrm{u}} \rightarrow \mathbb{B}\left(\mathcal{K}_{1}\right)$ such that $\left(\varphi \otimes \operatorname{id}_{A}\right) \tilde{V}=\mathrm{U}^{\mathcal{K}_{1}}$.

Similarly, the universal property 2.40 gives rise to a unique morphism $\psi: A^{\mathrm{u}} \rightarrow \mathbb{B}\left(\mathcal{K}_{2}\right)$ such that $\left(\operatorname{id}_{\hat{A}} \otimes \psi\right) \mathcal{V}=\mathrm{V}^{\mathcal{K}_{2}}$.

Let $(\pi, \hat{\pi})$ be a $\mathbb{G}$-Heisenberg pair on $\mathcal{H}_{\pi}$. Applying $\left(\varphi \otimes \operatorname{id}_{\mathcal{H}_{\pi}} \otimes \psi\right)$ on both sides of the defining condition (2.53) of the universal bicharacter $\mathcal{W} \in \mathcal{U}\left(\hat{A}^{\mathrm{u}} \otimes A^{\mathrm{u}}\right)$ gives

$$
((\varphi \otimes \psi) \mathcal{W})_{13}=\left(\mathrm{U}^{\mathcal{K}_{1}}\right)_{1 \pi}^{*} \mathrm{~V}_{\tilde{\pi} 3}^{\mathcal{K}_{2}} \mathrm{U}_{1 \pi}^{\mathcal{K}_{1}}\left(\mathrm{~V}^{\mathcal{K}_{2}}\right)_{\pi 3}^{*}
$$

Comparing the last expressions with (5.27) gives $Z=(\varphi \otimes \psi) \mathcal{W}^{*} \in \mathcal{U}\left(\mathcal{K}_{1} \otimes \mathcal{K}_{2}\right)$.

Following the proof of Proposition 5.14 we conclude that $\chi^{\mathcal{K}_{2}} \mathcal{K}^{\mathcal{K}_{1}}$ is symmetric if and only if $\sigma(\mathcal{W}) \mathcal{W}=$ $1 ;$ hence $\mathrm{W}=\widehat{\mathrm{W}}$.

Corollary 5.34. Assume a $\mathfrak{D}(\mathbb{G})^{\wedge}$-compatible pair $(\mathrm{U}, \mathrm{V})$ on a Hilbert space $\mathcal{H}$ and $Z \in \mathcal{U}(\mathcal{H} \otimes \mathcal{H})$ satisfy 2.30 . Then ${ }^{\mathcal{H}} \boldsymbol{X}^{\mathcal{H}}:=Z \circ \Sigma \in \mathcal{U}(\mathcal{H} \otimes \mathcal{H})$ is the braiding induced by $\mathfrak{D}(\mathbb{G})^{\wedge}$.

Remark 5.35. Example 5.29 provides a $\mathfrak{D}(\mathbb{G})^{\wedge}$-compatible pair $(\mathrm{U}, \mathrm{V})$ on $\mathcal{H}$ starting from a corepresentation $\mathrm{U} \in \mathcal{U}(\mathbb{K}(\mathcal{H}) \otimes A)$ of a quasitriangular quantum group $\mathbb{G}$. By Proposition 2.38 there is a unique $Z \in \mathcal{U}(\mathcal{H} \otimes \mathcal{H})$ satisfying the following commutation for the pair (V, $\mathrm{U})$,

$$
\mathrm{V}_{1 \hat{\eta}} \mathrm{U}_{2 \eta} Z_{12}=\mathrm{U}_{2 \eta} \mathrm{V}_{1 \hat{\eta}} \quad \text { in } \mathcal{U}\left(\mathcal{H} \otimes \mathcal{H} \otimes \mathcal{H}_{\eta}\right)
$$

for any $\mathbb{G}$-Heisenberg pair $(\eta, \hat{\eta})$ on $\mathcal{H}_{\eta}$. Given any $\mathbb{G}$-Heisenberg pair $(\pi, \hat{\pi})$ on $\mathcal{H}_{\pi}$ define an $\mathcal{R}$-Heisenberg pair $(\alpha, \beta)$ as in Lemma 4.11 Equations 5.28 and 5.37 yield:

$$
\mathrm{U}_{1 \alpha} \mathrm{U}_{2 \beta} Z_{12}=\mathrm{U}_{1 \pi} \mathrm{V}_{1 \hat{\eta}} \mathrm{U}_{2 \eta} Z_{12}=\mathrm{U}_{2 \eta} \mathrm{U}_{1 \pi} \mathrm{V}_{1 \hat{\eta}}=\mathrm{U}_{2 \beta} \mathrm{U}_{1 \alpha} .
$$

Finally, comparing the last equation with 5.19$)$ gives $Z^{(\mathcal{H}, \mathcal{H})}=Z$. Therefore, by Proposition 5.9 , the braiding ${ }^{\mathcal{H}} X^{\mathcal{H}}:=Z \circ \Sigma$ corresponds to the braiding induced by the $R$-matrix of $\mathbb{G}$.

There are two possible ways to define crossed products of $C_{1}$ and $C_{2}$. First, the $R$-matrix $\mathcal{R}$ on $\mathfrak{D}(\mathbb{G})$ allows to construct $C_{1} \otimes_{\mathcal{R}} C_{2}$. The second construction uses half of the available structures, the coactions $\gamma_{1}$ and $\delta_{2}$ of $\mathbb{G}$ and $\widehat{\mathbb{G}}$ on $C_{1}$ and $C_{2}$, to construct $C_{1} \otimes_{\mathrm{W}} C_{2}$. Proposition 4.55 shows that $\mathfrak{C}^{*} \mathfrak{a l g}\left(\mathfrak{D}(\mathbb{G})^{\wedge}\right)$ and $\mathcal{Y} \mathcal{D C} \mathfrak{C}^{*} \mathfrak{a l g}(\mathbb{G})$ are equivalent. In 30 , Nest and Voigt have shown that $\mathcal{Y} \mathcal{D C} \mathfrak{C}^{*} \mathfrak{a l g}(\mathbb{G})$ is endowed with a monoidal structure, assuming Haar weights on $\mathbb{G}$. We generalise their construction by showing that the monoidal structure on $\mathcal{Y} \mathcal{D} \mathfrak{C}^{*} \mathfrak{a l g}(\mathbb{G})$ gets induced from the braided monoidal structure on $\mathfrak{C o r e p}\left(\mathfrak{D}(\mathbb{G})^{\uparrow}\right)$.

Proposition 5.36. $\mathcal{Y} \mathcal{D C} \mathfrak{C}^{*} \mathfrak{a l g}(\mathbb{G})$ is a monoidal category.

Proof. Let $\left(\gamma_{i}, \delta_{i}\right)$ be a $\mathbb{G}$-Yetter-Drinfeld pair on the $\mathrm{C}^{*}$-algebra $C_{i}$ for $i=1,2$. Without loss of generality, we may assume covariant representations $\left(U^{\mathcal{K}_{i}}, \varphi_{i}\right)$ and $\left(\mathrm{V}^{\mathcal{K}_{i}}, \varphi_{i}\right)$ as in $(5.32)$. Hence the associated pair of corepresentations $\left(\mathrm{U}^{\mathcal{K}_{i}}, \mathrm{~V}^{\mathcal{K}_{i}}\right)$ satisfies the $\mathfrak{D}(\mathbb{G})^{\wedge}$-compatibility condition (5.27).

Given a $\mathbb{G}$-Heisenberg pair $(\eta, \hat{\eta})$ on a Hilbert space $\mathcal{L}$, let $Z \in \mathcal{U}\left(\mathcal{K}_{1} \otimes \mathcal{K}_{2}\right)$ satisfy 2.30):

$$
\mathrm{U}_{1 \eta}^{\mathcal{K}_{1}} \mathrm{~V}_{2 \eta}^{\mathcal{K}_{2}} Z_{12}=\mathrm{V}_{2 \hat{\eta}}^{\mathcal{K}_{2}} \mathrm{U}_{1 \eta}^{\mathcal{K}_{1}} \quad \text { in } \mathcal{U}\left(\mathcal{K}_{1} \otimes \mathcal{K}_{2} \otimes \mathcal{L}\right)
$$

Lemma 5.31 shows that ${ }^{\mathcal{K}_{2}} \chi^{\mathcal{K}_{1}}:=Z \circ \Sigma^{\mathcal{K}_{2}, \mathcal{K}_{1}}$ defines a braiding on $\operatorname{Corep}\left(\mathfrak{D}(\mathbb{G})^{\wedge}\right)$. Theorem 4.27 yields the following equivalence of crossed products

$$
C_{1} \otimes_{\mathrm{W}} C_{2} \stackrel{\Psi}{\cong}\left(\varphi_{1}\left(C_{1}\right) \otimes 1_{\mathcal{K}_{2}}\right) \cdot\left(\stackrel{\mathcal{K}_{2}}{X^{\mathcal{K}_{1}}}\right)\left(\varphi_{2}\left(C_{2}\right) \otimes 1_{\mathcal{K}_{1}}\right)\left(\stackrel{\mathcal{K}_{2}}{X^{\mathcal{K}_{1}}}\right)^{*} .
$$

Lemma 4.16 defines the embeddings $\iota_{C_{1}}\left(c_{1}\right):=\gamma_{1}\left(c_{1}\right)_{1 \eta}$ and $\iota_{C_{2}}\left(c_{2}\right):=\delta_{2}\left(c_{2}\right)_{2 \hat{\eta}}$ into $C_{1} \bigotimes_{\mathrm{W}} C_{2} \subset \mathcal{M}\left(C_{1} \otimes\right.$ $\left.C_{2} \otimes \mathbb{K}(\mathcal{L})\right)$ for $c_{i} \in C_{i}, i=1,2$. 
The unitary $Z$ on $\mathcal{U}\left(\mathcal{K}_{1} \otimes \mathcal{K}_{2}\right)$ satisfies (5.35). Hence, a similar computation as in the proofs of Theorem 5.17 and Proposition 5.18 gives a unique diagonal coaction $\gamma_{1} \bowtie_{\mathrm{W}} \gamma_{2}: C_{1} \otimes_{\mathrm{W}} C_{2} \rightarrow C_{1} \otimes_{\mathrm{W}} C_{2} \otimes A$ such that

$$
\left(\Psi \otimes \operatorname{id}_{A}\right)\left(\left(\gamma_{1} \bowtie_{\mathrm{W}} \gamma_{2}\right)\left(\iota_{C_{1}}\left(c_{1}\right) \iota_{C_{2}}\left(c_{2}\right)\right)=\left(\mathrm{U}^{\mathcal{K}_{1}} \oplus \mathrm{U}^{\mathcal{K}_{2}}\right)\left(\Psi\left(\iota_{C_{1}}\left(c_{1}\right) \iota_{C_{2}}\left(c_{2}\right)\right) \otimes 1_{A}\right)\left(\mathrm{U}^{\mathcal{K}_{1}} \oplus \mathrm{U}^{\mathcal{K}_{2}}\right)^{*} .\right.
$$

Hence $\left(\Psi, \mathrm{U}^{\mathcal{K}_{1}} \oplus \mathrm{U}^{\mathcal{K}_{2}}\right)$ is a covariant representation of $\left(C_{1} \otimes_{\mathrm{W}} C_{2}, \gamma_{1} \bowtie_{\mathrm{W}} \gamma_{2}, A\right)$.

Similarly, there is a unique coaction $\delta_{1} \bowtie_{\mathrm{W}} \delta_{2}: C_{1} \otimes_{\mathrm{W}} C_{2} \rightarrow C_{1} \otimes_{\mathrm{W}} C_{2} \otimes \hat{A}$ such that

$$
\left(\Psi_{1} \otimes \mathrm{id}_{A}\right)\left(\left(\delta_{1} \bowtie_{\mathrm{W}} \delta_{2}\right)\left(\iota_{C_{1}}\left(c_{1}\right) \iota_{C_{2}}\left(c_{2}\right)\right)=\left(\mathrm{V}^{\mathcal{K}_{1}} \oplus \mathrm{V}^{\mathcal{K}_{2}}\right)\left(\Psi_{1}\left(\iota_{C_{1}}\left(c_{1}\right) \iota_{C_{2}}\left(c_{2}\right)\right) \otimes 1_{\hat{A}}\right)\left(\mathrm{V}^{\mathcal{K}_{1}} \oplus \mathrm{V}^{\mathcal{K}_{2}}\right)^{*} .\right.
$$

Using the $\mathbb{G}$-Yetter-Drinfeld compatibility 5.26$)$ for the pair of coactions $\left(\gamma_{i}, \delta_{i}\right)$ we establish the same for the pair of diagonal coactions $\left(\gamma_{1} \bowtie_{\mathrm{W}} \gamma_{2}, \delta_{1} \bowtie_{\mathrm{W}} \delta_{2}\right)$ :

$$
\begin{aligned}
& \left(\left(\delta_{1} \nabla_{\mathrm{W}} \delta_{2}\right) \otimes \operatorname{id}_{A}\right)\left(\gamma_{1} \otimes_{\mathrm{W}} \gamma_{2}\right)\left(\iota_{C_{1}}\left(c_{1}\right) \iota_{C_{2}}\left(c_{2}\right)\right) \\
& =\left(\left(\delta_{1} \otimes_{\mathrm{W}} \delta_{2}\right) \otimes \mathrm{id}_{A}\right)\left(\left(\iota_{C_{1}} \otimes \mathrm{id}_{A}\right) \gamma_{1}\left(c_{1}\right)\left(\iota_{C_{2}} \otimes \mathrm{id}_{A}\right) \gamma_{2}\left(c_{2}\right)\right) \\
& =\left(\left(\iota_{C_{1}} \otimes \operatorname{id}_{\hat{A} \otimes A}\right)\left(\delta_{1} \otimes \operatorname{id}_{A}\right) \gamma_{1}\left(c_{1}\right)\right)\left(\left(\iota_{C_{2}} \otimes \operatorname{id}_{\hat{A} \otimes A}\right)\left(\delta_{2} \otimes \operatorname{id}_{A}\right) \gamma_{2}\left(c_{2}\right)\right) \\
& =\sigma_{23}^{\mathrm{W}}\left(\left(\left(\iota_{C_{1}} \otimes \mathrm{id}_{A \otimes \hat{A}}\right)\left(\gamma_{1} \otimes \operatorname{id}_{\hat{A}}\right) \delta_{1}\left(c_{1}\right)\right)\left(\left(\iota_{C_{2}} \otimes \operatorname{id}_{A \otimes \hat{A}}\right)\left(\gamma_{2} \otimes \operatorname{id}_{\hat{A}}\right) \delta_{2}\left(c_{2}\right)\right)\right) \\
& =\sigma_{23}^{\mathrm{W}}\left(\left(\gamma_{1} \nabla_{\mathrm{W}} \gamma_{2}\right) \otimes \operatorname{id}_{\hat{A}}\right)\left(\delta_{1} \otimes_{\mathrm{W}} \delta_{2}\right)\left(\iota_{C_{1}}\left(c_{1}\right) \iota_{C_{2}}\left(c_{2}\right)\right)
\end{aligned}
$$

for $c_{i} \in C_{i}, i=1,2$

Hence $C_{1} \bigotimes_{\mathrm{W}} C_{2} \in \mathcal{Y} \mathcal{D} \mathfrak{C}^{*} \mathfrak{a l g}(\mathbb{G})$ and $\Psi$ preserves $\mathbb{G}$-Yetter-Drinfeld covariant representations. Therefore, we may use an argument as in the proof of Theorem 5.16 to show that $\nabla_{\mathrm{W}}$ is associative.

\subsubsection{The dual coaction of a quantum group on itself}

The crossed product associated to a bicharacter is isomorphic to the standard tensor product when either of the coactions is inner (see Corollary 4.38). The dual of the reduced bicharacter $\widehat{\mathrm{W}} \in \mathcal{U}(\hat{A} \otimes A)$ of a quantum group $\mathbb{G}=\left(A, \Delta_{A}\right)$ induces a morphism $\hat{\delta}: A \rightarrow A \otimes \hat{A}$ defined by

$$
\hat{\delta}(a):=\widehat{\mathrm{W}}\left(a \otimes 1_{\hat{A}}\right) \widehat{\mathrm{W}}^{*} \quad \text { in } \mathcal{M}(A \otimes \hat{A}) \text { for } a \in A .
$$

Clearly, $\hat{\delta}$ is injective. The following computation shows that $\hat{\delta}$ is coassociative:

$$
\left(\operatorname{id}_{A} \otimes \hat{\Delta}_{A}\right) \hat{\delta}(a)=\widehat{\mathrm{W}}_{12} \widehat{\mathrm{W}}_{13}\left(a \otimes 1_{\hat{A}} \otimes 1_{\hat{A}}\right) \widehat{\mathrm{W}}_{13}^{*} \widehat{\mathrm{W}}_{12}^{*}=\left(\hat{\delta} \otimes \mathrm{id}_{\hat{A}}\right) \hat{\delta}(a)
$$

for $a \in A$.

Unfortunately, the Podlés condition 2.23 for the coaction $\hat{\delta}$ is not automatic.

Let $\mathbb{G}$ have a right Haar weight $\psi$. The multiplicative unitary $\mathbb{W} \in \mathcal{U}\left(\mathcal{H}_{\psi} \otimes \mathcal{H}_{\psi}\right)$ constructed in 24.25 is called the right regular representation of $\mathbb{G}$, where $\mathcal{H}_{\psi}$ is the GNS-space of $\psi$. Proposition 2.6 in 3 provides an equivalence between regularity for quantum groups and multiplicative unitaries. More precisely, a quantum group $\mathbb{G}$ is regular if and only if $A \rtimes_{r} \hat{A}$ is isomorphic to $\mathbb{K}\left(\mathcal{H}_{\psi}\right)$.

Definition 5.37. A quantum group $\mathbb{G}$ is weakly regular if

$$
\hat{A} \otimes A=\left\{\left(\hat{a} \otimes 1_{A}\right) \mathrm{W}\left(1_{\hat{A}} \otimes a\right): a \in A, \hat{a} \in \hat{A}\right\}^{\mathrm{CLS}} .
$$

Remark 5.38. The criterion 5.40 in Definition 5.37 has the merit of using only the pair $\left(A, \Delta_{A}\right)$. By Proposition 3.16 in 2], regular quantum groups are weakly regular.

Lemma 4.35 yields a necessary and sufficient condition for a quantum group to be weakly regular:

Corollary 5.39. A quantum group $\mathbb{G}$ is weakly regular if and only if the dual coaction $\hat{\delta}$ defined by (5.39) satisfies the Podlés condition. 
Proof. Let $R_{A}, R_{\hat{A}}$ be the unitary antipodes (involutive antiautomorphisms of $A$ and $\hat{A}$ ). By Proposition 3.10 application of $R_{\hat{A}} \otimes R_{A}$ to 5.40 yields an equivalent criterion:

$$
\hat{A} \otimes A=\left\{\left(1_{\hat{A}} \otimes a\right) \mathrm{W}\left(\hat{a} \otimes 1_{A}\right) a \in A, \hat{a} \in \hat{A}\right\}^{\mathrm{CLS}} .
$$

Finally, identifying $\gamma=a \otimes 1_{\hat{A}}$ and $u=\widehat{\mathrm{W}}$ in Lemma 4.35 completes the proof.

Therefore, in case of quantum groups with Haar weight, regularity implies Podlés condition 2.23 for $\hat{\delta}$. Let $(\pi, \hat{\pi})$ be a $\mathbb{G}$-Heisenberg pair on a Hilbert space $\mathcal{H}$. The following simple computation shows that $\left(\Delta_{A}, \hat{\delta}\right)$ is a $\mathbb{G}$-Yetter-Drinfeld pair:

$$
\sigma_{23}^{\mathrm{W}}\left(\left(\left(\pi \otimes \operatorname{id}_{A}\right) \Delta_{A} \otimes \operatorname{id}_{\hat{A}}\right) \hat{\delta}(a)\right)=\widehat{\mathrm{W}}_{\pi 2} \mathrm{~W}_{\hat{\pi} 3}\left(\pi(a) \otimes 1_{\hat{A} \otimes A}\right) \mathrm{W}_{\hat{\pi} 3}^{*} \widehat{\mathrm{W}}_{\pi 2}^{*}=\left(\left(\pi \otimes \operatorname{id}_{\hat{A}}\right) \hat{\delta} \otimes \operatorname{id}_{A}\right) \Delta_{A}(a) .
$$

for $a \in A$.

For $C \in \mathcal{Y} \mathcal{D C} \mathfrak{C}^{*} \mathfrak{a l g}(\mathbb{G})$, let $(\gamma, \hat{\gamma})$ be the $\mathbb{G}$-Yetter-Drinfeld structure on $C$. Construct the crossed product $\left(C \nabla_{\mathrm{W}} A, \iota_{C}, \iota_{A}\right)$ or equivalently $(C, \gamma) \bigotimes_{\mathrm{W}}(A, \hat{\delta})$. Therefore, we have $C \bigotimes_{\mathrm{W}} A \in \mathcal{Y} \mathcal{D} \mathfrak{C}^{*} \mathfrak{a l g}(\mathbb{G})$. Since $\hat{\delta}$ is an inner coaction of $\widehat{\mathbb{G}}$ on $A$, Corollary 4.38 gives an isomorphism $C \unrhd_{\mathrm{W}} A \cong C \otimes A$. This is not an isomorphism of crossed products.

Proposition 5.40. There is an isomorphism $\Psi: C \bigotimes_{\mathrm{W}} A \rightarrow C \otimes A$ of crossed products such that $\Psi \circ \iota_{C}(c)=$ $\gamma(c)$ and $\Psi \circ \iota_{A}(a)=1_{C} \otimes a$ for $c \in C$ and $a \in A$. Moreover, the map $\Psi \otimes \operatorname{id}_{A \otimes \hat{A}}$ induces a $\mathbb{G}$-Yetter-Drinfeld structure on $C \otimes A$.

Proof. Let $(\pi, \hat{\pi})$ be a $\mathbb{G}$-Heisenberg pair on a Hilbert space $\mathcal{H}$. By construction, $C \square_{\mathrm{W}} A \subseteq \mathcal{M}(C \otimes A \otimes \mathbb{K}(\mathcal{H}))$ and $\iota_{C}(c):=\gamma(c)_{1 \pi}, \iota_{A}(a):=\hat{\delta}(a)_{2 \hat{\pi}}$, for $c \in C, a \in A$. Then $\sigma_{23}^{\mathrm{W}_{\hat{\pi} 2}}: \mathcal{M}(C \otimes A \otimes \mathbb{K}(\mathcal{H})) \rightarrow \mathcal{M}(C \otimes \mathbb{K}(\mathcal{H}) \otimes A)$ maps $C \unrhd_{\mathrm{W}} A$ isomorphically to the crossed product generated by

$$
\left.\sigma_{23}^{\mathrm{W}_{\hat{\pi} 2}(\iota C}(c)\right)=\mathrm{W}_{\hat{\pi} 3} \gamma(c)_{1 \pi} \mathrm{W}_{\hat{\pi} 3}^{*}=\left(\left(\operatorname{id}_{C} \otimes \Delta_{A}\right) \gamma(c)\right)_{1 \pi 3}=\left(\left(\gamma \otimes \operatorname{id}_{A}\right) \gamma(c)\right)_{1 \pi 3}
$$

and

for $c \in C, a \in A$.

$$
\sigma_{23}^{\mathrm{W}_{\hat{\pi} 2}}\left(\iota_{A}(a)\right)=\mathrm{W}_{\hat{\pi} 3} \mathrm{~W}_{\pi 33}^{*}\left(1_{C} \otimes 1_{\mathcal{H}} \otimes a\right) \mathrm{W}_{\pi 33}^{*} \mathrm{~W}_{\hat{\pi} 3}=\left(\left(\gamma \otimes \operatorname{id}_{A}\right)(1 \otimes a)\right)_{1 \pi 3}
$$

The Podlés condition 2.23 for $\gamma$ yields $\left(\left(\gamma \otimes \operatorname{id}_{A}\right) \gamma(c)(1 \otimes a)\right)_{1 \pi 3}=\gamma(C)_{1 \pi} \otimes A \cong C \otimes A$.

Thus $\sigma_{23}^{\mathrm{W}} \mathrm{W}_{\tilde{\pi} 2}$ restricts to an isomorphism $\Psi$ of $\mathrm{C}^{*}$-algebras with the desired properties.

Notation 5.41. Given $C_{i} \in \mathcal{Y} \mathcal{D} \mathfrak{C}^{*} \mathfrak{a l g}(\mathbb{G})$, the canonical embedding of $C_{i}$ into $C_{1} \otimes_{\mathrm{W}} C_{2} \otimes_{\mathrm{W}} C_{3}$ is denoted by $j_{C_{i}}$ for $i=1,2,3$.

Let $D \in \mathcal{Y} \mathcal{D} \mathfrak{C}^{*} \mathfrak{a l g}(\mathbb{G})$ with the associated $\mathbb{G}$-Yetter-Drinfeld pair $(\lambda, \hat{\lambda})$, and consider the respective crossed products $\left(A \bigotimes_{\mathrm{W}} D, \iota_{A}, \iota_{D}\right),\left(C \bigotimes_{\mathrm{W}} D, \iota_{C}^{\prime}, \iota_{D}^{\prime}\right)$.

Corollary 5.42. There is an isomorphism $\Psi_{C, A, D}: C \otimes_{\mathrm{W}} A \bigotimes_{\mathrm{W}} D \rightarrow C \otimes\left(A \bigotimes_{\mathrm{W}} D\right)$ such that

$$
\Psi_{C, A, D} \circ j_{C}(c)=\left(\operatorname{id}_{C} \otimes \iota_{A}\right) \gamma(c), \quad \Psi_{C, A, D} \circ j_{A}(a)=1_{C} \otimes \iota_{A}(a), \quad \Psi_{C, A, D} \circ j_{D}(d)=1_{C} \otimes \iota_{D}(d) .
$$

Similarly, there is an isomorphism, $\Psi_{C, D, A}: C \bigotimes_{\mathrm{W}} D \bigotimes_{\mathrm{W}} A \rightarrow\left(C \bigotimes_{\mathrm{W}} D\right) \otimes A$ such that

$$
\Psi_{C, D, A} \circ j_{C}(c)=\left(\iota_{C}^{\prime} \otimes \mathrm{id}_{A}\right) \gamma(c), \quad \Psi_{C, D, A} \circ j_{D}(d)=\left(\iota_{D}^{\prime} \otimes \mathrm{id}_{A}\right) \lambda(d), \quad \Psi_{C, D, A} \circ j_{A}(a)=1_{C \bigotimes_{\mathrm{W}} D} \otimes a .
$$

Proof. Associativity of $\square_{\mathrm{W}}$ and Proposition 5.40 imply

$$
C \otimes_{\mathrm{W}} A \otimes_{\mathrm{W}} D \cong\left(C \otimes_{\mathrm{W}} A\right) \bigotimes_{\mathrm{W}} D \cong(C \otimes A) \bigotimes_{\mathrm{W}} D .
$$

Since the coaction of $\mathbb{G}$ on $C \otimes A$ is $\operatorname{id}_{C} \otimes \Delta_{A}$, Lemma 4.20 gives

$$
(C \otimes A) \bigotimes_{\mathrm{W}} D \cong C \otimes\left(A \otimes_{\mathrm{W}} D\right) .
$$

Composition of the isomorphisms gives $\Psi_{C, A, D}$ intertwining the embeddings of $C, A, D$.

Finally, 5.43 follows from Proposition 5.40 for $C \otimes_{\mathrm{W}} D$. 


\section{Chapter 6}

\section{Braided multiplicative unitaries}

In this final chapter we define a general theory of braided multiplicative unitaries and braided quantum groups using all the machinery developed in the preceding chapters. In Section 6.1 we define braided multiplicative unitaries and braided quantum groups. In Section 6.2, Theorem 6.7 shows that $\mathrm{C}^{*}$-quantum groups with projection canonically give rise to braided multiplicative unitaries. More precisely, we start with a $\mathrm{C}^{*}$-quantum group and a projection on it and then decompose its multiplicative unitary into two parts: standard and braided. We move on by showing that some known examples of $\mathrm{C}^{*}$-quantum groups are indeed $\mathrm{C}^{*}$-quantum groups with projection. Furthermore, we reconstruct a "big" standard multiplicative unitary from a given braided one (see Theorem 6.15. Proposition 6.17 shows that $\mathrm{C}^{*}$-quantum groups arise from these "big" multiplicative unitaries are again $\mathrm{C}^{*}$-quantum groups with projection. This is our "semidirect product"; hence we have generalised Radford's construction for multiplicative unitaries. In the final Section 6.4 use our theory to construct the simplified quantum $\mathrm{E}(2)$ by realising the quantum plane as braided a quantum group over the compact quantum group $\mathrm{C}(\mathbb{T})$.

\subsection{Braided quantum groups}

Let $\mathbb{G}=\left(A, \Delta_{A}\right)$ be a quantum group and let $\mathrm{W}^{A} \in \mathcal{U}(\hat{A} \otimes A)$ be its reduced bicharacter. Fix a $\mathbb{G}$-Heisenberg pair $(\eta, \hat{\eta})$ on a Hilbert space $\mathcal{H}$. Given any corepresentation $\mathrm{U} \in \mathcal{U}(\mathbb{K}(\mathcal{L}) \otimes A)$ of $\mathbb{G}$ on a Hilbert space $\mathcal{L}$, define $\mathbb{U}:=\left(\operatorname{id}_{\mathcal{L}} \otimes \eta\right) \mathrm{U} \in \mathcal{U}(\mathcal{L} \otimes \mathcal{H})$. For a corepresentation $\mathrm{V} \in \mathcal{U}(\mathbb{K}(\mathcal{L}) \otimes \hat{A})$ of $\widehat{\mathbb{G}}$ on $\mathcal{L}$, we define $\hat{\mathbb{V}}:=\Sigma\left(\left(\operatorname{id}_{\mathcal{L}} \otimes \hat{\eta}\right) \mathrm{V}^{*}\right) \Sigma \in \mathcal{U}(\mathcal{H} \otimes \mathcal{L})$.

Throughout this section we assume the pair $(\mathrm{U}, \mathrm{V})$ to be $\mathfrak{D}(\mathbb{G})^{\widehat{ }}$-compatible, that is,

$$
\mathbb{U}_{23} \mathbb{W}_{13}^{A} \hat{\mathbb{V}}_{12}=\hat{\mathbb{V}}_{12} \mathbb{W}_{13}^{A} \mathbb{U}_{23} \quad \text { in } \mathcal{U}(\mathcal{H} \otimes \mathcal{L} \otimes \mathcal{H})
$$

Proposition 2.38 gives a unique unitary $Z \in \mathcal{U}(\mathcal{L} \otimes \mathcal{L})$ characterised by (5.27), which we rewrite in the following equivalent form

$$
\hat{\mathbb{V}}_{23} \mathbb{U}_{12}=\mathbb{U}_{12} Z_{13}^{*} \hat{\mathbb{V}}_{23} \quad \text { in } \mathcal{U}(\mathcal{L} \otimes \mathcal{H} \otimes \mathcal{L}) .
$$

$$
\text { Following Corollary } 5.34 \text { we define a braiding operator }{ }^{\mathcal{L}} \chi^{\mathcal{L}} \text { by }{ }^{\mathcal{L}} \chi^{\mathcal{L}}:=Z \circ \Sigma \in \mathcal{U}(\mathcal{L} \otimes \mathcal{L}) \text {. }
$$

Definition 6.1. An element $\mathbb{F} \in \mathcal{U}(\mathcal{L} \otimes \mathcal{L})$ is called a braided multiplicative unitary over $\mathbb{G}$ if it satisfies

1. $\mathbb{G}$-invariance:

$$
\mathrm{U}_{13} \mathrm{U}_{23} \mathbb{F}_{12}=\mathbb{F}_{12} \mathrm{U}_{13} \mathrm{U}_{23} \quad \text { in } \mathcal{U}(\mathbb{K}(\mathcal{L} \otimes \mathcal{L}) \otimes A)
$$

2. $\widehat{\mathbb{G}}$-invariance:

$$
\hat{\mathrm{V}}_{13} \hat{\mathrm{V}}_{12} \mathbb{F}_{23}=\mathbb{F}_{23} \hat{\mathrm{V}}_{13} \hat{\mathrm{V}}_{12} \quad \text { in } \mathcal{U}(\hat{A} \otimes \mathbb{K}(\mathcal{L} \otimes \mathcal{L}))
$$


3. braided pentagon equation:

or equivalently

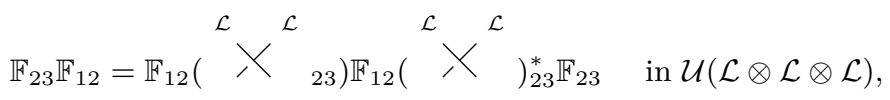

$$
\mathbb{F}_{23} \mathbb{F}_{12}=\mathbb{F}_{12} Z_{23} \mathbb{F}_{13} Z_{23}^{*} \mathbb{F}_{23} \quad \text { in } \mathcal{U}(\mathcal{L} \otimes \mathcal{L} \otimes \mathcal{L})
$$

Let us explain the leg numbering of the braided pentagon equation 6.6 in detail. The unitary $\mathrm{K}^{\mathcal{L}}{ }_{23}^{\mathcal{L}} \mathbb{F}_{12}\left({ }^{\mathcal{L}} \mathrm{X}^{\mathcal{L}}\right.$

$23)^{*}$ plays the role of $\mathbb{F}_{13}$. This is a straightforward generalisation of standard leg $\mathcal{L} \mathcal{L}$

numbering, replacing $\Sigma$ by the braiding operator $\backslash$. We have $\mathbb{F}_{23}=1_{\mathcal{L}} \otimes \mathbb{F}$ and $\mathbb{F}_{12}=\mathbb{F} \otimes 1_{\mathcal{L}}$, because $\mathbb{F}$ is invariant under the coactions of $\mathbb{G}$ and $\widehat{\mathbb{G}}$ implemented by the corepresentations $\mathrm{U}$ and $\hat{\mathrm{V}}$. We prove this by the following chain of computation:

$$
\begin{gathered}
\left\{\begin{array}{c}
\hat{\mathbb{V}}_{34} \mathbb{U}_{13} \mathbb{U}_{23} \mathbb{F}_{12} \\
=\hat{\mathbb{V}}_{34} \mathbb{F}_{12} \mathbb{U}_{13} \mathbb{U}_{23}
\end{array}\right\} \stackrel{1}{\Longrightarrow}\left\{\begin{array}{c}
\mathbb{U}_{13} Z_{14}^{*} \hat{\mathbb{V}}_{34} \mathbb{U}_{23} \mathbb{F}_{12} \\
=\mathbb{F}_{12} \mathbb{U}_{13} Z_{14}^{*} \hat{\mathbb{V}}_{34} \mathbb{U}_{23}
\end{array}\right\} \stackrel{2}{\Longrightarrow}\left\{\begin{array}{c}
\mathbb{U}_{13} Z_{14}^{*} \mathbb{U}_{23} Z_{24}^{*} \hat{\mathbb{V}}_{34} \mathbb{F}_{12} \\
=\mathbb{F}_{12} \mathbb{U}_{13} Z_{14}^{*} \mathbb{U}_{23} Z_{24}^{*} \hat{\mathbb{V}}_{34}
\end{array}\right\} \\
\stackrel{3}{\Longrightarrow}\left\{\begin{array}{c}
\mathbb{U}_{13} \mathbb{U}_{23} Z_{14}^{*} Z_{24}^{*} \mathbb{F}_{12} \hat{\mathbb{V}}_{34} \\
=\mathbb{F}_{12} \mathbb{U}_{13} \mathbb{U}_{23} Z_{14}^{*} Z_{24}^{*} \hat{\mathbb{V}}_{34}
\end{array}\right\} \stackrel{4}{\Longrightarrow}\left\{\begin{array}{c}
\mathbb{U}_{13} \mathbb{U}_{23} Z_{14}^{*} Z_{24}^{*} \mathbb{F}_{12} \\
=\mathbb{U}_{13} \mathbb{U}_{23} \mathbb{F}_{12} Z_{14}^{*} Z_{24}^{*}
\end{array}\right\} \stackrel{5}{\Longrightarrow}\left\{\begin{array}{c}
Z_{14}^{*} Z_{24}^{*} \mathbb{F}_{12} \\
=\mathbb{F}_{12} Z_{14}^{*} Z_{24}^{*}
\end{array}\right\}
\end{gathered}
$$

We begin with multiplication of $\hat{\mathbb{V}}_{34}$ on both sides of $(6.3), 1$ and 2 use 6.2 , 3 follows from the ordinary commutation of unitaries, 4 uses 6.3 and cancellation of $\mathbb{V}_{34}$ from both sides, 5 uses cancellation of $\mathbb{U}_{13} \mathbb{U}_{23}$

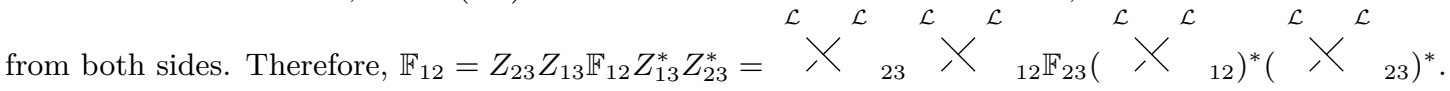
Remark 6.2. If either of the two corepresentations $(\mathrm{U}, \mathrm{V})$ is trivial then 6.2 implies $Z=1_{\mathcal{L} \otimes \mathcal{L}}$. In that case, 6.5 is the standard pentagon equation 2.4.

Let $\mathbb{G}=\left(A, \Delta_{A}\right)$ be a quasitriangular quantum group. Let $\Delta_{R}: A \rightarrow A \otimes \hat{A}$ be the right quantum group homomorphism associated to the $R$-matrix $\mathcal{R} \in \mathcal{U}(\hat{A} \otimes \hat{A})$. Let $\mathrm{U} \in \mathcal{U}(\mathbb{K}(\mathcal{L}) \otimes A)$ be a corepresentation of $\mathbb{G}$ on a Hilbert space $\mathcal{L}$. The right quantum group homomorphism $\Delta_{R}$ induce a dual corepresentation $\mathrm{V} \in$ $\mathcal{U}(\mathbb{K}(\mathcal{L}) \otimes \hat{A})$ characterised by 5.28$):\left(\mathrm{id}_{\mathcal{L}} \otimes \Delta_{R}\right) \mathrm{U}=\mathrm{U}_{12} \mathrm{~V}_{13}$.

Let $\mathrm{U}$ satisfies 6.3 . Applying $\operatorname{id}_{\mathcal{L} \otimes \mathcal{L}} \otimes \Delta_{R}$ on both sides of 6.3 gives

$$
\mathrm{U}_{13} \mathrm{~V}_{14} \mathrm{U}_{23} \mathrm{~V}_{24} \mathbb{F}_{12}=\mathbb{F}_{12} \mathrm{U}_{13} \mathrm{~V}_{14} \mathrm{U}_{23} \mathrm{~V}_{24} \quad \text { in } \mathcal{U}(\mathbb{K}(\mathcal{L} \otimes \mathcal{L}) \otimes A \otimes \hat{A}) .
$$

Using ordinary commutation between $\mathrm{U}_{23}, \mathrm{~V}_{14}$ and then using 6.3 on the right hand gives

$$
\mathrm{U}_{13} \mathrm{U}_{23} \mathrm{~V}_{14} \mathrm{~V}_{24} \mathbb{F}_{12}=\mathbb{F}_{12} \mathrm{U}_{13} \mathrm{U}_{23} \mathrm{~V}_{14} \mathrm{~V}_{24}=\mathrm{U}_{13} \mathrm{U}_{23} \mathbb{F}_{12} \mathrm{~V}_{14} \mathrm{~V}_{24} \quad \text { in } \mathcal{U}(\mathbb{K}(\mathcal{L} \otimes \mathcal{L}) \otimes A \otimes \hat{A}) .
$$

Hence $\mathbb{F} \in \mathcal{U}(\mathcal{L} \otimes \mathcal{L})$ is also $\widehat{\mathbb{G}}$-invariant for the induced dual corepresentation $\hat{\mathrm{V}}:=\sigma\left(\mathrm{V}^{*}\right) \in \mathcal{U}(\hat{A} \otimes \mathbb{K}(\mathcal{L}))$.

Remark 5.35 shows that $(\mathrm{U}, \mathrm{V})$ is a $\mathfrak{D}(\mathbb{G})^{\wedge}$-compatible pair. Hence the condition (6.1) is automatic for a braided multiplicative unitary over a quasitriangular quantum group $\mathbb{G}$.

We want to interpret braided multiplicative unitaries as a fundamental obejct to study braided quantum groups in complete generality. Keeping in mind the general theory of $\mathrm{C}^{*}$-quantum groups, in the sense of Woronowicz 49, we shall restrict our attention to the manageable braided multiplicative unitaries (see Definition 6.3). Following fact ought to be a theorem when $\mathbb{F}$ is manageable:

$$
K:=\left\{\left(\omega \otimes \operatorname{id}_{\mathcal{L}}\right) \mathbb{F}: \omega \in \mathbb{B}(\mathcal{L})_{*}\right\}^{\mathrm{CLS}}
$$

is a nondegenerate $\mathrm{C}^{*}$-subalgebra of $\mathbb{B}(\mathcal{L})$. Motivated by the known examples, we shall assume existence of some suitable $K \subset \mathbb{B}(\mathcal{L})$ to formulate our working definition of braided quantum group at the end of this section.

For the sake of convenience let us rewrite the corepresentation condition for 2.25 of $\mathrm{U}$ and $\hat{\mathrm{V}}$ following (6.1) and using the multiplicative unitary $\mathbb{W}^{A}:=(\hat{\eta} \otimes \eta) \mathrm{W}^{A} \in \mathcal{U}(\mathcal{H} \otimes \mathcal{H})$ as follows:

$$
\begin{aligned}
\mathbb{W}_{23}^{A} \mathbb{U}_{12}=\mathbb{U}_{12} \mathbb{U}_{13} \mathbb{W}_{23}^{A} & \text { in } \mathcal{U}(\mathcal{L} \otimes \mathcal{H} \otimes \mathcal{H}), \\
\hat{\mathbb{V}}_{23} \mathbb{W}_{12}^{A}=\mathbb{W}_{12}^{A} \hat{\mathbb{V}}_{13} \hat{\mathbb{V}}_{23} & \text { in } \mathcal{U}(\mathcal{H} \otimes \mathcal{H} \otimes \mathcal{L}) .
\end{aligned}
$$


Let $\mathbb{W}^{A} \in \mathcal{U}(\mathcal{H} \otimes \mathcal{H})$ be a manageable multiplicative unitary and let $Q_{A}$ and $\widetilde{\mathbb{W}^{A}} \in \mathcal{U}(\overline{\mathcal{H}} \otimes \mathcal{H})$ be the respective operators in Definition 2.10

Definition 6.3. A braided multiplicative unitary $\mathbb{F} \in \mathcal{U}(\mathcal{L} \otimes \mathcal{L})$ is said to be manageable if there are a positive, self-adjoint operator $Q_{K}$ on $\mathcal{L}$ with $\operatorname{Ker}\left(Q_{K}\right)=\{0\}$ and $\widetilde{\mathbb{F}} \in \mathcal{U}(\overline{\mathcal{L}} \otimes \mathcal{L})$ such that

1. $\mathbb{U}\left(Q_{K} \otimes Q_{A}\right) \mathbb{U}^{*}=Q_{K} \otimes Q_{A}$.

2. $\mathbb{V}^{*}\left(Q_{K} \otimes Q_{A}\right) \mathbb{V}=Q_{K} \otimes Q_{A}$ or $\hat{\mathbb{V}}^{*}\left(Q_{A} \otimes Q_{K}\right) \hat{\mathbb{V}}=Q_{A} \otimes Q_{K}$.

3. $\mathbb{F}\left(Q_{K} \otimes Q_{K}\right) \mathbb{F}^{*}=Q_{K} \otimes Q_{K}$.

4. $\left(x \otimes u\left|Z^{*} \mathbb{F}\right| y \otimes v\right)=\left(\bar{y} \otimes Q_{K}(u)|\widetilde{\mathbb{F}}| \bar{x} \otimes Q_{K}^{-1}(v)\right)$ for any $x, y \in \mathcal{L}, u \in \mathcal{D} \operatorname{Dom}\left(Q_{K}\right)$ and $v \in \mathcal{D}$ om $\left(Q_{K}^{-1}\right)$.

Lemma 6.4. Let $\mathbb{V} \in \mathcal{U}(\mathcal{L} \otimes \hat{A})$ and $Z \in \mathcal{U}(\mathcal{L} \otimes \mathcal{L})$ be as in 6.2. Then

$$
Z^{*}\left(Q_{K} \otimes Q_{K}\right) Z=Q_{K} \otimes Q_{K}
$$

Proof. Using the commutation relations 11 and 2 we obtain 6.10 from the following computation:

$$
\begin{aligned}
Z_{13}\left(Q_{K} \otimes Q_{A} \otimes Q_{K}\right) Z_{13}^{*} & =\mathbb{U}_{12}^{*} \hat{\mathbb{V}}_{23} \mathbb{U}_{12} \hat{\mathbb{V}}_{23}^{*}\left(Q_{K} \otimes Q_{A} \otimes Q_{K}\right) \hat{\mathbb{V}}_{23} \mathbb{U}_{12}^{*} \hat{\mathbb{V}}_{23}^{*} \mathbb{U}_{12} \\
& =Q_{K} \otimes Q_{A} \otimes Q_{K} .
\end{aligned}
$$

Define $\Delta_{K}: \mathbb{K}(\mathcal{L}) \rightarrow \mathbb{B}(\mathcal{L} \otimes \mathcal{L})$ by

$$
\Delta_{K}(\zeta):=\mathbb{F}\left(\zeta \otimes 1_{\mathcal{L}}\right) \mathbb{F}^{*} \quad \text { for } \zeta \in \mathbb{K}(\mathcal{L}) .
$$

If the coactions of $\mathbb{G}$ and $\widehat{\mathbb{G}}$ on $\mathbb{K}(\mathcal{L})$ implemented by corepresentations $\mathrm{U}$ and $\mathrm{V}$ satisfy Podleś condition 2.23 , Example 4.39 gives $\mathbb{K}(\mathcal{L}) \bigotimes_{\mathrm{W}} \mathbb{K}(\mathcal{L}) \cong \mathbb{K}(\mathcal{L} \otimes \mathcal{L})$; hence $\Delta_{K}$ is an element in $\operatorname{Mor}\left(\mathbb{K}(\mathcal{L}), \mathbb{K}(\mathcal{L}) \bigotimes_{\mathrm{W}}\right.$ $\mathbb{K}(\mathcal{L}))$.

The general theory of crossed products gives $K \otimes_{\mathrm{W}^{A}} K \cong\left(K \otimes 1_{\mathcal{L}}\right) \cdot Z\left(1_{\mathcal{L}} \otimes K\right) Z^{*}$. The braided pentagon equation 6.6 implies

$$
\mathbb{F}_{23} \mathbb{F}_{12} \mathbb{F}_{23}^{*}=\mathbb{F}_{12} Z_{12} \mathbb{F}_{13} Z_{12}^{*} \quad \text { in } \mathcal{U}\left(\mathbb{K}(\mathcal{L}) \otimes K \otimes_{\mathrm{W}} K\right)
$$

Let us assume (6.7) holds for a manageable $\mathbb{F} \in \mathcal{U}(\mathcal{L} \otimes \mathcal{L})$. Then slicing the first leg on both sides of the last equation by $\omega \in \mathbb{B}(\mathcal{L})$ we obtain the braided comultiplication $\Delta_{K}: K \rightarrow \mathcal{M}\left(K \nabla_{\mathrm{W}} K\right)$ defined by $\Delta_{K}(k):=\mathbb{F}\left(k \otimes 1_{\mathcal{L}}\right) \mathbb{F}^{*}$. Moreover, the braided pentagon equation confirms the coassociativity of $\Delta_{K}$ :

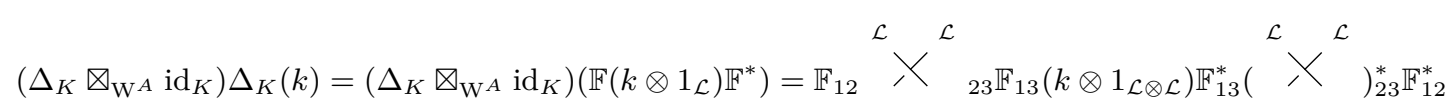

$$
\begin{aligned}
& =\mathbb{F}_{23} \mathbb{F}_{12}\left(k \otimes 1_{\mathcal{L} \otimes \mathcal{L}}\right) \mathbb{F}_{12}^{*} \mathbb{F}_{23}^{*}=\left(\operatorname{id}_{K} \otimes_{\mathrm{W}^{A}} \Delta_{K}\right) \Delta_{K}(k) \text {. }
\end{aligned}
$$

This motivates the following Definition:

Definition 6.5. Let $\mathbb{F} \in \mathcal{U}(\mathcal{L} \otimes \mathcal{L})$ be a braided, manageable multiplicative unitary. A pair $\left(K, \Delta_{K}\right)$ is called a braided quantum group over $\mathbb{G}$ if

1. $\mathbb{F} \in \mathcal{U}(\mathbb{K}(\mathcal{L}) \otimes K)$,

2. $K \in \mathcal{Y} \mathcal{D} \mathfrak{C}^{*} \mathfrak{a l g}(\mathbb{G})$ with the inner coactions induced by $\mathrm{U}$ and $\mathrm{V}$,

3. the comultiplication $\Delta_{K}: K \rightarrow K \nabla_{\mathrm{W}^{A}} K$ is implemented by $\mathbb{F}$ :

$$
\Delta_{K}(k)=\mathbb{F}\left(k \otimes 1_{K}\right) \mathbb{F}^{*} \quad \text { for } k \in K .
$$




\subsection{Quantum groups with projection}

In this section, we show that every quantum group with projection canonically gives rise to a braided multiplicative unitary. Let $\mathbb{I}=\left(C, \Delta_{C}\right)$ be a quantum group with the reduced bicharacter $\mathrm{W}^{C} \in \mathcal{U}(\hat{C} \otimes C)$. Given a projection $\mathrm{P} \in \mathcal{U}(\hat{C} \otimes C)$ on $\mathbb{G}$ let $\mathbb{G}=\left(A, \Delta_{A}\right)$ be its image. Consider the left quantum group homomorphism $\Delta_{L}: C \rightarrow C \otimes C$ associated to the projection $\mathrm{P}$.

For a given $\mathbb{I}$-Heisenberg pair $(\pi, \hat{\pi})$ on $\mathcal{H}_{\pi}$ and the associated $\mathbb{I}$-anti-Heisenberg pair $(\bar{\pi}, \overline{\hat{\pi}})$ on $\overline{\mathcal{H}}_{\pi}$, define the following representations of $C$ and $\hat{C}$ on $\mathcal{L}:=\overline{\mathcal{H}}_{\pi} \otimes \mathcal{H}_{\pi}$ :

$$
\hat{\rho}(\hat{c}):=(\overline{\hat{\pi}} \otimes \hat{\pi}) \hat{\Delta}_{C}(\hat{c}), \quad \rho(c):=(\bar{\pi} \otimes \pi) \Delta_{L}(c), \quad \text { and } \quad \pi^{\prime}(c):=\left(1_{\overline{\mathcal{H}}_{\pi}} \otimes \pi(c)\right) .
$$

Lemma 6.6. Define the corepresentations $\mathrm{U}:=\left(\hat{\rho} \otimes \mathrm{id}_{A}\right) \mathrm{P} \in \mathcal{U}(\mathbb{K}(\mathcal{L}) \otimes A)$ of $\mathbb{G}$ and $\hat{\mathrm{V}}:=\left(\operatorname{id}_{\hat{A}} \otimes \rho\right) \mathrm{P} \in$ $\mathcal{U}(\hat{A} \otimes \mathbb{K}(\mathcal{L}))$ of $\widehat{\mathbb{G}}$ acting on $\mathcal{L}$.

1. The pair $(\mathrm{U}, \mathrm{V})$ is $\mathfrak{D}(\mathbb{G})^{\wedge}$-compatible. Equivalently, $\mathrm{U}$ and $\hat{\mathrm{V}}$ commute in the following way:

$$
\mathrm{U}_{23} \mathrm{P}_{13} \hat{\mathrm{V}}_{12}=\hat{\mathrm{V}}_{12} \mathrm{P}_{13} \mathrm{U}_{23} \quad \text { in } \mathcal{U}(\hat{A} \otimes \mathbb{K}(\mathcal{L}) \otimes A)
$$

with the identification $\mathrm{W}^{A}=\mathrm{P} \in \mathcal{U}(\hat{A} \otimes A)$.

2. Given any $\mathbb{G}$-Heisenberg pair $(\eta, \hat{\eta})$ on $\mathcal{H}_{\eta}$, the unitary $Z:=(\hat{\rho} \otimes \rho) \mathrm{P}^{*} \in \mathcal{U}(\mathcal{L} \otimes \mathcal{L})$ solves the following equation:

$$
\begin{array}{rl}
\hat{\mathrm{V}}_{\hat{\eta} 3} \mathrm{U}_{1 \eta} & =\mathrm{U}_{1 \eta} Z_{13}^{*} \hat{\mathrm{V}}_{\hat{\eta} 3} \quad \text { in } \mathcal{U}\left(\mathcal{L} \otimes \mathcal{H}_{\eta} \otimes \mathcal{L}\right) . \\
\mathcal{L} & \mathcal{L} \\
\text { Equivalently, the operator } & X^{\mathcal{L}}:=Z \circ \Sigma \in \mathcal{U}(\mathcal{L} \otimes \mathcal{L}) \text { defines a braiding. }
\end{array}
$$

3. $\left(\hat{\rho}, \pi^{\prime}\right)$ is an $\mathbb{I}$-Heisenberg on $\mathcal{L}$, that is,

$$
\mathrm{W}_{\hat{\rho} 3}^{C} \mathrm{~W}_{1 \pi^{\prime}}^{C}=\mathrm{W}_{1 \pi^{\prime}}^{C} \mathrm{~W}_{13}^{C} \mathrm{~W}_{\hat{\rho} 3}^{C} \quad \text { in } \mathcal{U}(\hat{C} \otimes \mathbb{K}(\mathcal{L}) \otimes C)
$$

Proof. Lemma 3.40 implies $\left.\hat{\Delta}_{C}\right|_{\hat{A}}=\hat{\Delta}_{A}$ and $\left.\Delta_{L}\right|_{A}=\Delta_{A}$. We also may identify $\mathrm{W}^{A}$ with P. Then Corollary 4.15 implies 6.13 .

The pentagon equation 3.28 for $\mathrm{P}$ yields $\mathrm{P}_{\hat{\eta} 3} \mathrm{P}_{1 \eta}=\mathrm{P}_{1 \eta} \mathrm{P}_{13} \mathrm{P}_{\hat{\eta} 3}$. Applying $\hat{\rho} \otimes \mathrm{id}_{\mathcal{H}_{\eta}} \otimes \rho$ on both sides of the last expression gives 6.14 .

Using the Heisenberg commutation relation 2.12 of the pair $(\pi, \hat{\pi})$, we compute

$$
\mathrm{W}_{\hat{\pi} 4}^{C} \mathrm{~W}_{\bar{\pi} 4}^{C} \mathrm{~W}_{1 \pi}^{C}=\mathrm{W}_{\hat{\pi} 4}^{C} \mathrm{~W}_{1 \pi}^{C} \mathrm{~W}_{\overline{\tilde{\pi}} 4}^{C}=\mathrm{W}_{1 \pi}^{C} \mathrm{~W}_{14}^{C} \mathrm{~W}_{\hat{\pi} 4}^{C} \mathrm{~W}_{\bar{\pi} 4}^{C} .
$$

The character condition 2.9 on the first leg of $\mathrm{W}^{C}$ shows that the last equation is equivalent to 6.15.

Theorem 6.7. Let $\mathbb{I}=\left(C, \Delta_{C}\right)$ be a $\mathrm{C}^{*}$-quantum group with a projection $\mathrm{P} \in \mathcal{U}(\hat{C} \otimes C)$. The the second leg of the unitary

$$
\mathrm{F}=\mathrm{P}^{*} \mathrm{~W}^{C} \in \mathcal{U}(\hat{C} \otimes C)
$$

is invariant under the left quantum group homomorphism $\Delta_{L}: C \rightarrow C \otimes C$ associated to the projection $\mathrm{P}$. Let $\mathbb{W}^{C}:=(\hat{\pi} \otimes \pi) \mathrm{W}^{C} \in \mathcal{U}\left(\mathcal{H}_{\pi} \otimes \mathcal{H}_{\pi}\right)$ be a manageable multiplicative unitary. Then $\mathbb{F}:=(\hat{\rho} \otimes \rho) \mathrm{F} \in$ $\mathcal{U}(\mathcal{L} \otimes \mathcal{L})$ is a manageable, braided multiplicative unitary with respect to the $\mathfrak{D}(\mathbb{G})^{\uparrow}$-compatible pair $(\mathrm{U}, \mathrm{V})$ of corepresentations on $\mathcal{L}$.

The proof uses the following lemma on commutation relations between $\mathrm{P}$ and $\mathrm{F}$.

Lemma 6.8. Let $(\eta, \hat{\eta})$ be an $\mathbb{I}$-Heisenberg pair on a Hilbert space $\mathcal{H}_{\eta}$. Then

$$
\begin{aligned}
\mathrm{P}_{13} \mathrm{P}_{\hat{\eta} 3} \mathrm{~F}_{1 \eta} & =\mathrm{F}_{1 \eta} \mathrm{P}_{13} \mathrm{P}_{\hat{\eta} 3} & & \text { in } \mathcal{U}\left(\hat{C} \otimes \mathbb{K}\left(\mathcal{H}_{\eta}\right) \otimes C\right), \\
\mathrm{P}_{1 \eta}^{*} \mathrm{~F}_{\hat{\eta} 3} & =\mathrm{F}_{\hat{\eta} 3} \mathrm{P}_{1 \eta}^{*} & & \text { in } \mathcal{U}\left(\hat{C} \otimes \mathbb{K}\left(\mathcal{H}_{\eta}\right) \otimes C\right) .
\end{aligned}
$$


Proof. Using $(\eta, \hat{\eta})$ we rewrite 3.3$)$ as

$$
\mathrm{W}_{\hat{\eta}_{3}}^{C} \mathrm{P}_{1 \eta}=\mathrm{P}_{1 \eta} \mathrm{P}_{13} \mathrm{~W}_{\hat{\eta} 3}^{C} \quad \text { in } \mathcal{U}\left(\hat{A} \otimes \mathbb{K}\left(\mathcal{H}_{\eta}\right) \otimes C\right) .
$$

Firstly,

$$
\mathrm{P}_{13} \mathrm{P}_{\hat{\eta} 3} \mathrm{~F}_{1 \eta}=\mathrm{P}_{13} \mathrm{P}_{\hat{\eta} 3} \mathrm{P}_{1 \eta}^{*} \mathrm{~W}_{1 \eta}^{C}=\mathrm{P}_{1 \eta}^{*} \mathrm{P}_{\hat{\eta} 3} \mathrm{~W}_{1 \eta}^{C}=\mathrm{P}_{1 \eta}^{*} \mathrm{~W}_{1 \eta}^{C} \mathrm{P}_{13} \mathrm{P}_{\hat{\eta} 3}=\mathrm{F}_{1 \eta} \mathrm{P}_{13} \mathrm{P}_{\hat{\eta} 3} .
$$

The first equality uses the definition of $\mathrm{F}$ in $(6.16)$, the second equality uses the pentagon equation 3.28 ) for $\mathrm{P}$, the third equality uses the bicharacter condition 6.19, and the fourth equality again uses 6.16).

Secondly,

$$
\mathrm{P}_{1 \eta}^{*} \mathrm{~F}_{\hat{\eta} 3}=\mathrm{P}_{1 \eta}^{*} \mathrm{P}_{\hat{\eta} 3}^{*} \mathrm{~W}_{\hat{\eta} 3}^{C}=\mathrm{P}_{\hat{\eta} 3}^{*} \mathrm{P}_{13}^{*} \mathrm{P}_{1 \eta}^{*} \mathrm{~W}_{\hat{\eta} 3}^{C}=\mathrm{P}_{\hat{\eta} 3}^{*} \mathrm{~W}_{\hat{\eta} 3}^{C} \mathrm{P}_{1 \eta}^{*}=\mathrm{F}_{\hat{\eta} 3} \mathrm{P}_{1 \eta}^{*} .
$$

The first equality follows from 6.16, the second equality uses the pentagon equation 3.28 , the third equality uses 6.19), and the last equality again uses 6.16).

Proof of Theorem 6.7. In order to show that $\mathbb{F} \in \mathcal{U}(\mathcal{L} \otimes \mathcal{L})$ is a braided multiplicative unitary, we must show the following:

$$
\begin{aligned}
\mathbb{F}_{23} \mathbb{F}_{12} & =\mathbb{F}_{12} Z_{23} \mathbb{F}_{13} Z_{23}^{*} \mathbb{F}_{23} & & \text { in } \mathcal{U}(\mathcal{L} \otimes \mathcal{L} \otimes \mathcal{L}), \\
\mathrm{U}_{13} \mathrm{U}_{23} \mathbb{F}_{12} & =\mathbb{F}_{12} \mathrm{U}_{13} \mathrm{U}_{23} & & \text { in } \mathcal{U}(\mathbb{K}(\mathcal{L} \otimes \mathcal{L}) \otimes A), \\
\hat{\mathrm{V}}_{13} \hat{\mathrm{V}}_{12} \mathbb{F}_{23} & =\mathbb{F}_{23} \hat{\mathrm{V}}_{13} \hat{\mathrm{V}}_{12} & & \text { in } \mathcal{U}(\hat{A} \otimes \mathbb{K}(\mathcal{L} \otimes \mathcal{L})),
\end{aligned}
$$

where $\mathrm{U}, \hat{\mathrm{V}}$ and $Z$ are same as in Lemma 6.6

Equation 3.19$]$ gives

$$
\left(\operatorname{id}_{\hat{C}} \otimes \Delta_{L}\right) \mathrm{W}^{C}=\mathrm{P}_{12} \mathrm{~W}_{13}^{C} \quad \text { in } \mathcal{U}(\hat{C} \otimes C \otimes C) .
$$

Equation 3.32 shows that $\left.\Delta_{L}\right|_{A}=\Delta_{A}$. Therefore, we get

$$
\left(\operatorname{id}_{\hat{C}} \otimes \Delta_{L}\right) \mathrm{F}=\left(\left(\operatorname{id}_{\hat{A}} \otimes \Delta_{A}\right) \mathrm{P}^{*}\right)\left(\operatorname{id}_{\hat{C}} \otimes \Delta_{L}\right) \mathrm{W}^{C}=\mathrm{P}_{13}^{*} \mathrm{P}_{12}^{*} \mathrm{P}_{12} \mathrm{~W}_{13}^{C}=\mathrm{F}_{13},
$$

where the second equality uses 6.23 and 3.31 and the last equality follows from the definition 6.16 of $\mathrm{F}$.

Hence the second leg of $\mathrm{F}$ is $\Delta_{L}$-invariant. To establish the braided pentagon equation for $\mathrm{F}$, we compute:

$$
\begin{aligned}
\mathrm{F}_{1 \eta} \mathrm{P}_{\hat{\eta} 3}^{*} \mathrm{~F}_{13} \mathrm{P}_{\hat{\eta} 3} \mathrm{~F}_{\hat{\eta} 3}=\mathrm{P}_{\hat{\eta} 3}^{*} \mathrm{P}_{13}^{*} \mathrm{~F}_{1 \eta} \mathrm{P}_{13} \mathrm{~F}_{13} \mathrm{P}_{\hat{\eta} 3} \mathrm{~F}_{\hat{\eta} 3}=\mathrm{P}_{\hat{\eta} 3}^{*} \mathrm{P}_{13}^{*} \mathrm{P}_{1 \eta}^{*} \mathrm{~W}_{1 \eta}^{C} \mathrm{~W}_{13}^{C} \mathrm{~W}_{\hat{\eta} 3}^{C} \\
\quad=\mathrm{P}_{1 \eta}^{*} \mathrm{P}_{\hat{\eta} 3}^{*} \mathrm{~W}_{\hat{\eta} 3}^{C} \mathrm{~W}_{1 \eta}^{C}=\mathrm{P}_{1 \eta}^{*} \mathrm{~F}_{\hat{\eta} 3} \mathrm{~W}_{1 \eta}^{C}=\mathrm{F}_{\hat{\eta} 3} \mathrm{P}_{1 \eta}^{*} \mathrm{~W}_{1 \eta}^{C}=\mathrm{F}_{\hat{\eta} 3} \mathrm{~F}_{1 \eta} .
\end{aligned}
$$

The first equality uses the first auxiliary relation (6.17), the second equality follows from (6.16), the third equality uses the Heisenberg commutation relations $(2.12)$ for $\mathrm{P}$ and $\mathrm{W}^{C}$, the fourth equality uses (6.16), the fifth equality uses the second auxiliary relation $\sqrt{6.18)}$ and the sixth equality again uses $(6.16)$.

The invariance condition (6.24) leads to the following observation:

$$
\left(\operatorname{id}_{\hat{C}} \otimes \rho\right) \mathrm{F}=\left(\operatorname{id}_{\hat{C}} \otimes(\eta \otimes \pi) \Delta_{L}\right) \mathrm{F}=\mathrm{F}_{1 \pi}=\left(\operatorname{id}_{\hat{C}} \otimes \pi^{\prime}\right) \mathrm{F} .
$$

Equation 6.15 shows that $\left(\hat{\rho}, \pi^{\prime}\right)$ is an $\mathbb{I}$-Heisenberg pair. We replace $(\eta, \hat{\eta})$ by $\left(\hat{\rho}, \pi^{\prime}\right)$ in 6.25. Keeping 6.26 in mind, we get

$$
\mathrm{F}_{1 \pi^{\prime}} \mathrm{P}_{\hat{\rho} 3}^{*} \mathrm{~F}_{13} \mathrm{P}_{\hat{\rho} 3} \mathrm{~F}_{\hat{\rho} 3}=\mathrm{F}_{\hat{\rho} 3} \mathrm{~F}_{1 \pi^{\prime}} \quad \text { in } \mathcal{U}(\hat{A} \otimes \mathbb{K}(\mathcal{L}) \otimes A) .
$$

Applying $\left(\hat{r} \otimes \operatorname{id}_{\mathcal{L}} \otimes \rho\right)$ on both sides of $(6.27)$ yields 6.20$)$.

Similarly, replacing $(\eta, \hat{\eta})$ by $\left(\hat{\rho}, \pi^{\prime}\right)$ in (6.17) and observing 6.26$)$, we get

$$
\mathrm{P}_{13} \mathrm{P}_{\hat{\rho} 3} \mathrm{~F}_{1 \rho}=\mathrm{P}_{13} \mathrm{P}_{\hat{\rho} 3} \mathrm{~F}_{1 \pi^{\prime}}=\mathrm{F}_{1 \pi^{\prime}} \mathrm{P}_{13} \mathrm{P}_{\hat{\rho} 3}=\mathrm{F}_{1 \rho} \mathrm{P}_{13} \mathrm{P}_{\hat{\rho} 3} \quad \text { in } \mathcal{U}(\hat{A} \otimes \mathbb{K}(\mathcal{L}) \otimes A) .
$$

Then application of $\left(\hat{\rho} \otimes \operatorname{id}_{\mathcal{L}} \otimes \rho\right)$ on both sides of 6.28$)$ yields 6.21 . 
Recall the identification $\chi^{A \rightarrow C}=\mathrm{P} \in \mathcal{U}(\hat{A} \otimes C)$ in (3.37). Since $\left.\Delta_{L}\right|_{A}=\Delta_{A}$, Proposition 4.14 shows that the pair of representations $(\hat{\rho}, \rho)$ is a $\chi^{A \rightarrow C}$-Drinfeld pair. Therefore, $(\hat{\rho}, \rho)$ satisfies 4.8, which is equivalent to

$$
\mathrm{W}_{\hat{\rho} 4}^{C} \mathrm{P}_{14} \mathrm{P}_{1 \rho}=\mathrm{P}_{1 \rho} \mathrm{P}_{14} \mathrm{~W}_{\hat{\rho} 4}^{C} \quad \text { in } \mathcal{U}(\hat{A} \otimes \mathbb{K}(\mathcal{L}) \otimes C) .
$$

Using 6.16, 6.13 and 6.29, we obtain

$$
\mathrm{P}_{14} \mathrm{P}_{1 \rho} \mathrm{F}_{\hat{\rho} 4}=\mathrm{P}_{14} \mathrm{P}_{1 \rho} \mathrm{P}_{\hat{\rho} 4}^{*} \mathrm{~W}_{\hat{\rho} 4}^{C}=\mathrm{P}_{\hat{\rho} 4}^{*} \mathrm{P}_{1 \rho} \mathrm{P}_{14} \mathrm{~W}_{\hat{\rho} 4}^{C}=\mathrm{F}_{\hat{\rho} 4} \mathrm{P}_{14} \mathrm{P}_{1 \rho} .
$$

Applying $\left(\hat{\rho} \otimes \mathrm{id}_{\mathcal{L}} \otimes \rho\right)$ on both sides of 6.30 gives 6.22). A simple observation shows $Z^{*} \mathbb{F}=(\hat{\rho} \otimes \rho)\left(\mathrm{P}^{*} \mathrm{~F}\right)=$ $(\hat{\rho} \otimes \rho)\left(\mathrm{W}^{C}\right)$. Let $Q_{C}$ and $\widetilde{\mathbb{W}^{C}} \in \mathcal{U}\left(\overline{\mathcal{H}}_{\pi} \otimes \mathcal{H}_{\pi}\right)$ be the operators in Definition 2.10 defining manageability of $\mathbb{W}^{C}=(\hat{\pi} \otimes \pi) \mathrm{W}^{C} \in \mathcal{U}\left(\mathcal{H}_{\pi} \otimes \mathcal{H}_{\pi}\right)$. with $\hat{\mathbb{V}}$.

Let $Q:=Q_{C}^{\top} \otimes Q_{C}$. Then $Q \otimes Q$ commutes with $\mathbb{F}, Q \otimes Q_{C}$ commutes with $\mathbb{U}$, and $Q_{C} \otimes Q$ commutes

A routine computation, similar to the proof of Theorem 6.16 , shows that the unitary $\widetilde{\mathbb{F}} \in \mathcal{U}(\overline{\mathcal{L}} \otimes \mathcal{L})$ defined by

$$
\widetilde{\mathbb{F}}:=\left(\left(\mathbb{W}^{C}\right)^{*}\right)_{13}^{R_{\hat{C}} \otimes \mathrm{T}}\left(\mathbb{W}_{14}^{C}\right)^{*}\left(\left(\mathbb{W}^{C}\right)^{*}\right)_{23}^{\mathrm{T} \otimes \mathrm{T}} \widetilde{\mathbb{W}^{C}}{ }_{24},
$$

where $R_{\hat{C}}$ is the unitary antipode of $\widehat{\mathbb{I}}$, satisfies the last condition in 6.3 .

Hence, the manageability of $\mathbb{F}$ follows from the manageability of $\mathbb{W}^{C}$.

Next we reconstruct a manageable multiplicative unitary generating $\mathbb{I}$ from $\mathrm{P}$ and $\mathrm{F}$. This is a special case of the general Theorems 6.15 and 6.16 of the next Section.

Proposition 6.9. Let $(\xi, \hat{\xi})$ be a $\mathbb{G}$-Heisenberg pair on a Hilbert space $\mathcal{H}$. Then

$$
\mathbb{W}_{1234}:=\mathbb{P}_{13} \mathbb{U}_{23} \hat{\mathbb{V}}_{34}^{*} \mathbb{F}_{24} \hat{\mathbb{V}}_{34} \quad \text { in } \mathcal{U}(\mathcal{H} \otimes \mathcal{L} \otimes \mathcal{H} \otimes \mathcal{L})
$$

is a multiplicative unitary generating $\mathbb{I}=\left(C, \Delta_{C}\right)$, where $\mathbb{P}=(\hat{\xi} \otimes \xi) \mathrm{P} \in \mathcal{U}(\mathcal{H} \otimes \mathcal{H}), \mathbb{U}=\left(\operatorname{id}_{\mathcal{L}} \otimes \xi\right) \mathrm{U} \in \mathcal{U}(\mathcal{L} \otimes \mathcal{H})$ and $\hat{\mathbb{V}}=\left(\hat{\xi} \otimes \operatorname{id}_{\mathcal{L}}\right) \hat{\mathrm{V}} \in \mathcal{U}(\mathcal{H} \otimes \mathcal{L})$.

Proof. By Lemma 6.6 (3) we know $\left(\hat{\rho}, \pi^{\prime}\right)$ is an $\mathbb{I}$-Heisenberg pair on $\mathcal{L}$. Since $\left.\hat{\Delta}_{C}\right|_{\hat{A}}=\hat{\Delta}_{A},\left(\hat{\rho}, \pi^{\prime}\right)$ is also an $\mathbb{G}$-Heisenberg pair on $\mathcal{L}$. We rewrite $(3.28)$ as

$$
\mathrm{P}_{1 \pi^{\prime}}^{*} \mathrm{U}_{23} \mathrm{P}_{1 \pi^{\prime}}=\mathrm{P}_{13} \mathrm{U}_{\hat{\rho} 3} \quad \text { in } \mathcal{U}(\hat{A} \otimes \mathbb{K}(\mathcal{L}) \otimes A)
$$

Equation 6.32 implies

$$
\mathrm{P}_{1 \xi} \mathrm{U}_{2 \xi} \hat{\mathbb{V}}_{34}^{*} \mathbb{F}_{24} \hat{\mathbb{V}}_{34}=\mathrm{P}_{1 \pi^{\prime}}^{*} \mathrm{U}_{2 \xi} \mathrm{P}_{1 \pi^{\prime}} \hat{\mathrm{V}}_{\hat{\xi} 4}^{*} \mathbb{F}_{24} \hat{\mathrm{V}}_{\hat{\xi} 4}=\mathrm{P}_{1 \pi^{\prime}}^{*} \mathrm{U}_{2 \xi} \hat{\mathrm{V}}_{\hat{\xi} 4}^{*} \mathrm{P}_{1 \pi^{\prime}} \mathbb{F}_{24} \hat{\mathrm{V}}_{\hat{\xi} 4}
$$

Now 6.14 and 6.30 imply

$$
\mathrm{P}_{1 \pi^{\prime}}^{*} \mathrm{U}_{2 \xi} \hat{\mathrm{V}}_{\hat{\xi} 4}^{*} \mathrm{P}_{1 \pi^{\prime}} \mathbb{F}_{24} \hat{\mathrm{V}}_{\hat{\xi} 4}=\mathrm{P}_{1 \pi^{\prime}}^{*} \hat{\mathrm{V}}_{\xi 4}^{*} \mathrm{U}_{2 \xi} Z_{24}^{*} \mathbb{F}_{24} \mathrm{P}_{1 \pi^{\prime}} \hat{\mathrm{V}}_{\hat{\xi} 4}
$$

The defining condition 6.16 gives $Z^{*} \mathbb{F}=(\hat{\rho} \otimes \rho) \mathrm{W}^{C}$. Using 6.23 we get

$\mathrm{P}_{1 \pi^{\prime}}^{*} \hat{\mathrm{V}}_{\hat{\xi} 4}^{*} \mathrm{U}_{2 \xi} Z_{24}^{*} \mathbb{F}_{24} \mathrm{P}_{1 \pi^{\prime}} \hat{\mathrm{V}}_{\hat{\xi} 4}=\mathrm{P}_{1 \pi^{\prime}}^{*} \hat{\mathrm{V}}_{\hat{\xi} 4}^{*} \mathrm{U}_{2 \xi}\left((\hat{r} \otimes r) \mathrm{W}^{C}\right)_{24} \mathrm{P}_{1 \pi^{\prime}} \hat{\mathrm{V}}_{\hat{\xi} 4}=\mathrm{P}_{1 \pi^{\prime}}^{*} \hat{\mathrm{V}}_{\hat{\xi} 4}^{*}\left(\left(\hat{\rho} \otimes(\xi \otimes \rho) \Delta_{L}\right) \mathrm{W}^{C}\right)_{234} \mathrm{P}_{1 \pi^{\prime}} \hat{\mathrm{V}}_{\hat{\xi} 4}$.

Define $\hat{\Pi}: \hat{C} \rightarrow \mathbb{B}(\mathcal{H} \otimes \mathcal{L})$ and $\Pi: C \rightarrow \mathbb{B}(\mathcal{H} \otimes \mathcal{L})$ by

$$
\Pi(c):=\mathrm{P}_{\hat{\xi} s}^{*}\left(1_{\mathcal{H}} \otimes \hat{r}(\hat{c})\right) \mathrm{P}_{\hat{\xi} s} \quad \text { and } \quad \hat{\Pi}(\hat{c}):=\hat{\mathbb{V}}^{*}\left((\xi \otimes r) \Delta_{L}(c)\right) \hat{\mathbb{V}}
$$

for $c \in C$ and $\hat{c} \in \hat{C}$.

Theorems 6.15 and 6.16 ensure that $\mathbb{W}_{1234}:=(\hat{\Pi} \otimes \Pi) \mathrm{W}^{C} \in \mathcal{U}(\mathcal{H} \otimes \mathcal{L} \otimes \mathcal{H} \otimes \mathcal{L})$ is a manageable multiplicative unitary. Therefore, $(\Pi, \hat{\Pi})$ is an $\mathbb{I}$-Heisenberg pair acting on $\mathcal{H} \otimes \mathcal{L}$; hence $\mathbb{W}_{1234}$ is a multiplicative unitary generating $\mathbb{I}$. 
The second leg of the unitary $\overline{\mathrm{F}}:=\mathrm{W}^{C} \mathrm{P}^{*} \in \mathcal{U}(\hat{C} \otimes C)$ is invariant under the right quantum group homomorphism $\Delta_{R}: C \rightarrow C \otimes C$ induced by P. By virtue of equation 3.33 we can replace $\rho$ by $\rho^{\prime}:=$ $(\bar{\pi} \otimes \pi) \Delta_{R}$. The operator ${ }^{\mathcal{L}} \chi^{\mathcal{L}}:=Z^{*} \circ \Sigma$ is also a braiding.

A similar technique as in the proof of Lemma 6.8 shows the following commutation relations:

Lemma 6.10. Let $(\eta, \hat{\eta})$ be any $\mathbb{I}$-Heisenberg pair acting on the Hilbert space $\mathcal{H}_{\eta}$. Then

$$
\begin{aligned}
\mathrm{P}_{1 \eta} \mathrm{P}_{13} \overline{\mathrm{F}}_{\hat{\eta} 3} & =\overline{\mathrm{F}}_{\hat{\eta} 3} \mathrm{P}_{1 \eta} \mathrm{P}_{13} & & \text { in } \mathcal{U}\left(\hat{C} \otimes \mathbb{K}\left(\mathcal{H}_{\eta}\right) \otimes C\right), \\
\mathrm{P}_{\hat{\eta} 3}^{*} \overline{\mathrm{F}}_{1 \eta} & =\overline{\mathrm{F}}_{1 \eta} \mathrm{P}_{\hat{\eta} 3}^{*} & & \text { in } \mathcal{U}\left(\hat{C} \otimes \mathbb{K}\left(\mathcal{H}_{\eta}\right) \otimes C\right) .
\end{aligned}
$$

Now using Lemma 6.10 we see that $\overline{\mathbb{F}} \in \mathcal{U}(\mathcal{L} \otimes \mathcal{L})$ is also a braided multiplicative unitary in the following sense:

1. $\overline{\mathbb{F}}$ is $\mathbb{G}$-invariant:

$$
\mathrm{U}_{13} \mathrm{U}_{23} \overline{\mathbb{F}}_{12}=\overline{\mathbb{F}}_{12} \mathrm{U}_{13} \mathrm{U}_{23} \quad \text { in } \mathcal{U}(\mathbb{K}(\mathcal{L} \otimes \mathcal{L}) \otimes A) .
$$

2. $\overline{\mathbb{F}}$ is $\widehat{\mathbb{G}}$-invariant:

$$
\hat{\mathrm{V}}_{13} \hat{\mathrm{V}}_{12} \overline{\mathbb{F}}_{23}=\overline{\mathbb{F}}_{23} \hat{\mathrm{V}}_{13} \hat{\mathrm{V}}_{12} \quad \text { in } \mathcal{U}(\hat{A} \otimes \mathbb{K}(\mathcal{L} \otimes \mathcal{L})) .
$$

3. $\overline{\mathbb{F}}$ satisfies the braided pentagon equation:

$$
\overline{\mathbb{F}}_{23} \overline{\mathbb{F}}_{12}=\overline{\mathbb{F}}_{12} Z_{12}^{*} \overline{\mathbb{F}}_{13} Z_{12} \overline{\mathbb{F}}_{23} \quad \text { in } \mathcal{U}(\mathcal{L} \otimes \mathcal{L} \otimes \mathcal{L}) .
$$

In order to define manageability for $\overline{\mathbb{F}}$ we modify Definition 6.3 slightly. We replace condition (4) in Definition 6.3 by

$$
(x \otimes u|\overline{\mathbb{F}} Z| y \otimes v)=\left(\bar{y} \otimes Q_{K}(u)|\widetilde{\overline{\mathbb{F}}}| \bar{x} \otimes Q_{K}^{-1}(v)\right),
$$

where $\widetilde{\mathbb{F}} \in \mathcal{U}(\overline{\mathcal{L}} \otimes \mathcal{L})$ for any $x, y \in \mathcal{L}, u \in \mathcal{D} \operatorname{om}\left(Q_{K}\right)$ and $v \in \mathcal{D} \operatorname{Dom}\left(Q_{K}^{-1}\right)$.

Thus we have an interplay between braided multiplicative unitaries for left and right invariant elements: $\mathrm{PF}=\overline{\mathrm{F}} \mathrm{P}=\mathrm{W}^{C} \in \mathcal{U}(\hat{C} \otimes C)$.

\subsubsection{Examples of quantum groups with projections}

Example 6.11 (Idempotents on groups). Let $I$ be a locally compact group. Example 3.38 shows that any projection on $\left(\mathrm{C}_{0}(I), \Delta_{\mathrm{C}_{0}(I)}\right)$ comes from an idempotent group homomorphism $p: I \rightarrow I$. Define $G:=\operatorname{im}(p)$ and $K:=\operatorname{ker}(p)$. Then $K$ is a normal subgroup of $I, G$ is a subgroup of $I$, and $I$ is homeomorphic to $G \times K$ via the multiplication map $(g, k) \rightarrow g k$ for $g \in G, k \in K$. There is a natural left action of $G$ on $I$. The homogeneous space $G / I$ is homeomorphic to $K$.

The dual (left and right) action of $G$ (or coaction of $\left.\left(\mathrm{C}_{\mathrm{r}}^{*}(G), \Delta_{\mathrm{C}_{\mathrm{r}}^{*}(G)}\right)\right)$ on $G / I$ is trivial; hence the braiding operator is just the standard flip. Hence $\mathrm{C}_{0}(G) \otimes \mathrm{C}_{0}(G / I) \cong \mathrm{C}_{0}(G \times G / I) \cong \mathrm{C}_{0}(G \times K) \cong \mathrm{C}_{0}(I)$ and $\mathrm{C}_{0}(K) \otimes \mathrm{C}_{0}(K) \cong \mathrm{C}_{0}(K \times K)$.

Among the known examples of $\mathrm{C}^{*}$-quantum groups, the quantum $\mathrm{E}(2)$ group by Woronowicz 51, the quantum $a z+b$ group by Woronowicz [50], and the quantum $a x+b$ group by Woronowicz and Zakrzewski [55], are of the form $\mathbb{F} \cdot \mathbb{P} \in \mathcal{U}(\mathcal{H} \otimes \mathcal{H})$. Classically, they are semidirect products of groups. We show that they are $\mathrm{C}^{*}$-quantum groups with projection. In those examples we show that every projection $\mathrm{P} \in \mathcal{U}(\hat{C} \otimes C)$ on $\mathbb{I}=\left(C, \Delta_{C}\right)$ is induced by an idempotent Hopf ${ }^{*}$-homomorphisms $f: C \rightarrow C$. Moreover, $\mathbb{F} \in \mathcal{U}(\mathcal{H} \otimes \mathcal{H})$ is implemented by quantum exponential functions, discussed in 52 .

Let $\mathcal{C}(\mathcal{H})$ denote the set of closed operator on a Hilbert space $\overline{\mathcal{H}}$. Given any $N \in \mathcal{C}(\mathcal{H})$ consider its polar decomposition $N=\operatorname{Phase}(N)|N|$, where $\operatorname{Phase}(N)$ is a partial isometry and $|N|$ is a positive self-adjoint operator on $\mathcal{H}$. If $\operatorname{ker}(N)=\{0\}$ then $\operatorname{Phase}(N)$ is unitary. If $N$ is normal then $\operatorname{Phase}(N)$ and $|N|$ commute.

Let $R, S \in \mathcal{C}(\mathcal{H})$ satisfying some commutation relations such that: $R$ and $S$ are normal and the spectrum of $R$ and $S$ is contained in some $\Lambda \subset \mathbb{C}$. Assume $R+S \in \mathcal{C}(\mathcal{H})$, normal and satisfying the same spectral condition. Let $\mathbb{T}:=\{z \in \mathbb{C}:|z|=1\}$. A continuous function $F: \Lambda \rightarrow \mathbb{T}$ is called a quantum exponential function if

$$
F(R+S)=F(R) F(S)
$$


In particular, let $(R, S)$ be a pair of commuting self-adjoint operators. More precisely, $R$ and $S$ are self-adjoint, such that $|R|$ and $|S|$ strongly commute. Then $\Lambda=\mathbb{R}$, and the function $F: \mathbb{R} \rightarrow \mathbb{T}$ satisfying 6.36 are the classical exponential functions $F_{t}(r)=\exp (\mathrm{i} t r)$ parametrised by $t \in \mathbb{R}$.

Let $0<q<1$ and define the sets

$$
\mathbb{C}_{(q)}:=q^{\mathbb{Z}} \cdot \mathbb{T} \quad \text { and } \quad \overline{\mathbb{C}}_{(q)}:=\mathbb{C}_{(q)} \cup\{0\} .
$$

Geometrically, $\mathbb{C}_{(q)}$ is a set of concentric circles in the complex plane centered at 0 , and $\overline{\mathbb{C}}_{(q)}$ is the closure of $\mathbb{C}_{(q)}$ containing the origin.

Let $R, S \in \mathcal{C}(\mathcal{H})$ such that $R$ and $S$ are normal, $\operatorname{Sp}(R), \operatorname{Sp}(S) \in \overline{\mathbb{C}}_{(q)}$, and $S R=q^{2} R S$. In this case, a quantum exponential function $\mathrm{F}_{q} \in C\left(\overline{\mathbb{C}}_{(q)}\right)$ satisfying $(6.36)$ is defined by

$$
\mathrm{F}_{q}(t):= \begin{cases}\prod_{k=1}^{\infty} \frac{1+q^{2 k} \bar{z}}{1+q^{2 k}} & z \in \overline{\mathbb{C}}_{(q)} \backslash\left\{-q^{2 \mathbb{Z}}\right\}, \\ -1 & \text { otherwise. }\end{cases}
$$

Clearly, $\left|\mathrm{F}_{q}(\eta)\right|=1$ for any $\eta \in \mathbb{C}_{(q)}$.

\subsubsection{Quantum E(2) group}

Let

$$
G^{\prime}=\left\{\left(\begin{array}{cc}
v & n \\
0 & v^{-1}
\end{array}\right): v, n \in \mathbb{C} \text { and }|v|=1\right\} .
$$

For any $z \in \mathbb{C}$ the map $z \mapsto v^{2} z+v n$ defines a homomorphism from $G^{\prime}$ onto the group of orientation and Euclidean distance preserving transformations of $\mathbb{C}$. The kernel of this homomorphism is isomorphic to $\mathbb{Z}_{2}$; hence $G^{\prime}$ is a double cover of the group of motions of the two dimensional Euclidean plane.

Let $0<q<1$ be the deformation parameter. Let $C^{\prime}$ be the ${ }^{*}$-algebra generated by $v$ and $n$ subject to the following relations

$$
v^{*} v=v v^{*}=1, \quad n^{*} n=n n^{*}, \quad \text { and } \quad v n v^{*}=q n .
$$

We denote the algebraic tensor product by $\odot$. Next we define the comulitplication $\Delta_{C^{\prime}}: C^{\prime} \rightarrow C^{\prime} \odot C^{\prime}$ on generators of $C^{\prime}$ :

$$
\Delta_{C^{\prime}}(v):=v \odot v \quad \text { and } \quad \Delta_{C^{\prime}}(n):=v \odot n+n \odot v^{*} .
$$

Clearly, $\Delta_{C^{\prime}}$ is coassociative. Woronowicz described its $\mathrm{C}^{*}$-algebraic version, denoted by $\mathrm{E}_{q}(2)$ in 47,51 . In order to pass to the $\mathrm{C}^{*}$-algebra level, first we realise $v$ as unitary and $n$ as normal, unbounded operator on some Hilbert space $\mathcal{H}$.

There is a natural action of $\mathbb{Z}$ on $\mathrm{C}_{0}\left(\overline{\mathbb{C}}_{(q)}\right)$ : for any $k \in \mathbb{Z}$ and $f \in \mathrm{C}_{0}\left(\overline{\mathbb{C}}_{(q)}\right)$ we define

$$
\left(q_{k} f\right)(z):=f\left(q^{k} z\right) \quad \text { for all } z \in \overline{\mathbb{C}}_{(q)} .
$$

Let $C^{\prime}=\mathrm{C}_{0}\left(\overline{\mathbb{C}}_{(q)}\right) \rtimes \mathbb{Z}$ be the corresponding crossed product. The ${ }^{*}$-homomorphism $\mathrm{C}_{0}\left(\overline{\mathbb{C}}_{(q)}\right) \hookrightarrow C^{\prime}$ is nondegenerate, and the generator of $\mathbb{Z}$ in $C^{\prime}$ is a unitary $v \in \mathcal{M}\left(C^{\prime}\right)$ with

$$
v f v^{*}=q_{1} f \quad \text { for all } f \in \mathrm{C}_{0}\left(\overline{\mathbb{C}}_{(q)}\right) .
$$

Define the function $n$ on $\mathrm{C}_{0}\left(\overline{\mathbb{C}}_{(q)}\right)$ by

$$
n(z)=z \quad \text { for all } z \in \overline{\mathbb{C}}_{(q)} .
$$

Let $X$ be a locally compact Hausdorff space. The set of all elements affiliated, in the sense of [47, Definition 1.1], to $\mathrm{C}_{0}(X)$ is $C(X)$. Therefore, $n$ is affiliated to $\mathrm{C}_{0}\left(\overline{\mathbb{C}}_{(q)}\right)$, and due to the nondegenerate embedding of $\mathrm{C}_{0}\left(\overline{\mathbb{C}}_{(q)}\right)$ into $C^{\prime}, n$ is affiliated to $C^{\prime}$. Finally, $n$ is normal, $\operatorname{Sp}(n) \subset \overline{\mathbb{C}}_{(q)}$ and $v n v^{*}=q n$.

The underlying $\mathrm{C}^{*}$-algebra of $\mathrm{E}_{q}(2)$, again denoted by $C^{\prime}$, is characterised by requiring $v, n$ affiliated to $C^{\prime}$ with

$$
v^{*} v=v v^{*}=1, \quad n^{*} n=n n^{*}, \quad \operatorname{Sp}(n) \subset \overline{\mathbb{C}}_{(q)} \quad \text { and } v n v^{*}=q n .
$$


Concretely, we can write 6.42 in terms of the following operator domains:

$$
\begin{aligned}
\mathcal{E}_{\mathcal{H}} & :=\left\{N \in \mathcal{C}(\mathcal{H}): \begin{array}{l}
N^{*} N=N N^{*} \\
\operatorname{Sp}(N) \subset \overline{\mathbb{C}}_{(q)}
\end{array}\right\}, \\
\mathcal{G}_{\mathcal{H}}^{\prime} & :=\left\{(v, n) \in \mathcal{U}(\mathcal{H}) \times \mathcal{E}_{\mathcal{H}}: v n v^{*}=q n\right\} .
\end{aligned}
$$

Therefore, $C^{\prime}$ is generated by a pair $(v, n) \in \mathcal{G}_{\mathcal{H}}^{\prime}$.

Moreover, $C^{\prime}$ is universal in the following sense: for any $\mathrm{C}^{*}$-algebra $D^{\prime}$ such that $V, N$ are affiliated to $D^{\prime}$ satisfying 6.42 there is a unique $\phi \in \operatorname{Mor}\left(C^{\prime}, D^{\prime}\right)$ such that $\phi(v)=V$ and $\phi(n)=N$. By construction, the spectrum of $n$ is restricted to a subset of $\overline{\mathbb{C}}_{(q)}$. The reason behind this is to give meaning to the comulitplication map $\Delta_{C^{\prime}} \in \operatorname{Mor}\left(C^{\prime}, C^{\prime} \otimes C^{\prime}\right)$ (see [47, Theorem 3.1]) on the generators $v$ and $n$ :

$$
\Delta_{C^{\prime}}(v)=v \otimes v \quad \text { and } \quad \Delta_{C^{\prime}}(n)=v \otimes n+n \otimes v^{*},
$$

where $\dot{+}$ denotes the closure of the sum. More precisely, $\Delta_{C^{\prime}}(n)$ is affiliated to $C^{\prime} \otimes C^{\prime}$ and $\operatorname{Sp}(n) \subset \mathbb{C}_{(q)}$ plays a crucial role to see this.

The dual of $\mathrm{E}_{q}(2)$ is the quantum deformation of the group of triangular matrices

$$
\hat{G}^{\prime}:=\left\{\left(\begin{array}{cc}
a^{-1} & 0 \\
b & a
\end{array}\right): a \in \mathbb{R}, a>0 \text { and } b \in \mathbb{C}\right\} .
$$

Define the operator domain

$$
\hat{\mathcal{G}}_{\mathcal{H}}^{\prime}:=\left\{(\hat{N}, b) \in \mathcal{C}(\mathcal{H}) \times \mathcal{E}_{\mathcal{H}}: \begin{array}{c}
\hat{N}=\hat{N}^{*}, \operatorname{Sp}(\hat{N}) \subset \mathbb{Z}, \quad \hat{N} \text { and }|b| \text { strongly commute } \\
\operatorname{Sp}(\hat{N},|b|) \subset \bar{\Sigma}_{q}, \text { on } \operatorname{ker}(b)^{\perp}:(\text { Phase }(b))^{*} \hat{N} \text { Phase }(b)=\hat{N}+2 I
\end{array}\right\},
$$

where $\bar{\Sigma}_{q}$ is the closure of the set $\Sigma_{q}:=\left\{\left(s, q^{r}\right): s, r-\frac{s}{2} \in \mathbb{Z}\right\}$.

The underlying $\mathrm{C}^{*}$-algebra of $\hat{\mathrm{E}}_{q}(2)$, denoted by $\hat{C}^{\prime}$, is the universal $\mathrm{C}^{*}$-algebra generated by a pair $(\hat{N}, b) \in \hat{\mathcal{G}}_{\mathcal{H}}^{\prime}$.

The dual comutiplication $\hat{\Delta}_{C^{\prime}}: \hat{C}^{\prime} \rightarrow \hat{C}^{\prime} \otimes \hat{C}^{\prime}$ on the generators is given by

$$
\hat{\Delta}_{C^{\prime}}(\hat{N}):=\hat{N} \otimes 1+1 \otimes \hat{N} \quad \text { and } \quad \hat{\Delta}_{C^{\prime}}(b):=b \otimes q^{\hat{N} / 2} \dot{+} q^{-\hat{N} / 2} \otimes b .
$$

Given a unitary $v$ and a self adjoint operator $\hat{N}$ with integer spectrum recall

$$
(1 \otimes v)^{\hat{N} \otimes 1}:=\int_{\mathbb{Z} \times \mathbb{T}} z^{s} \mathrm{~d} E_{\hat{N}}(s) \otimes \mathrm{d} E_{v}(z),
$$

where $\mathrm{d} E_{\hat{N}}(\cdot)$ and $\mathrm{d} E_{v}$ are the spectral measures of $\hat{N}$ and $u$, respectively.

Let $\pi: C^{\prime} \rightarrow \mathbb{B}(\mathcal{H})$ and $\hat{\pi}: \hat{C}^{\prime} \rightarrow \mathbb{B}(\mathcal{H})$ for some Hilbert space $\mathcal{H}$. For simplicity, we write $v, n, \hat{N}$ and $b$ instead of $\pi(v), \pi(n), \hat{\pi}(\hat{N})$ and $\hat{\pi}(b)$, respectively.

Theorem 6.12 ([51, Section 5]). The unitary

$$
\mathbb{W}:=\mathrm{F}_{q}\left(q^{\hat{N} / 2} b \otimes v n\right)(1 \otimes v)^{\hat{N} \otimes 1} \quad \text { in } \mathcal{U}(\mathcal{H} \otimes \mathcal{H})
$$

is a multiplicative unitary for $\mathrm{E}_{q}(2)$ if and only if

1. $(v, n) \in \mathcal{G}_{\mathcal{H}}^{\prime}$ and $(\hat{N}, b) \in \hat{\mathcal{G}}_{\mathcal{H}}^{\prime}$,

2. $v^{*} \hat{N} v=\hat{N}+1$,

3. $v b v^{*}=q^{-1 / 2} b$,

4. $\hat{N}$ and $|n|$ strongly commute,

5. $b n^{*}=q^{1 / 2} n^{*} b$,

6. $n b-q^{1 / 2} b n=\left(1-q^{2}\right) q^{-(\hat{N}+1) / 2} v$.

This is equivalent to $(\pi, \hat{\pi})$ being a $\mathrm{E}_{q}(2)$-Heisenberg pair on $\mathcal{H}$. 


\section{Projection on quantum E(2)}

The Hopf *-homomorphism $f: C^{\prime} \rightarrow C^{\prime}$ defined by $f(v):=v$ and $f(n):=0$ is a projections on $\mathrm{E}_{q}(2)$. Since $\mathrm{F}_{q}(0)=1$, applying $f$ on the second leg of the multiplicative unitary in 6.46) gives a bicharacter $\mathrm{P}$ defined by

$$
\mathrm{P}:=(\mathrm{id} \otimes f) \mathrm{F}_{q}\left(q^{\hat{N} / 2} b \otimes v n\right)(1 \otimes v)^{\hat{N} \otimes 1}=(1 \otimes v)^{\hat{N} \otimes 1} \quad \text { in } \mathcal{U}(\mathcal{H} \otimes \mathcal{H}) .
$$

The commutation relation $v^{*} \hat{N} v=\hat{N}+1$ shows that $\mathrm{P}$ is a projection on $\mathrm{E}_{q}(2)$. Equivalently, $\mathrm{P}$ satisfies the pentagon equation 2.4 :

$$
\mathrm{P}_{23} \mathrm{P}_{12} \mathrm{P}_{23}^{*}=(1 \otimes 1 \otimes v)^{1 \otimes \hat{N} \otimes 1}(1 \otimes v \otimes 1)^{\hat{N} \otimes 1 \otimes 1}\left(1 \otimes 1 \otimes v^{*}\right)^{1 \otimes \hat{N} \otimes 1}=(1 \otimes v \otimes v)^{\hat{N} \otimes 1 \otimes 1}=\mathrm{P}_{12} \mathrm{P}_{13} .
$$

The second leg in the above computation is realized on a Hilbert space associated to a $\mathrm{E}_{q}(2)$-Heisenberg pair.

Next we define the right quantum group homomorphism $\Delta_{R}:=\left(\operatorname{id}_{C^{\prime}} \otimes f\right) \Delta_{C^{\prime}}$. The comultiplication formula 6.45) shows that $\Delta_{R}(v n)=v n \otimes 1$. Since the second leg of $\overline{\mathrm{F}}=\mathrm{F}_{q}\left(q^{\hat{N} / 2} b \otimes v n\right)$ is $\Delta_{R}$-invariant, Equation (3.16) yields (6.34). The commutation relations in Theorem (6.12) and realisation of the second leg on a Hilbert space, implies

$$
\begin{aligned}
& (1 \otimes v \otimes 1)^{\hat{N} \otimes 1 \otimes 1}(1 \otimes 1 \otimes v)^{\hat{N} \otimes 1 \otimes 1}\left(1 \otimes q^{\hat{N} / 2} b \otimes v n\right) \\
& =(1 \otimes v \otimes 1)^{\hat{N} \otimes 1 \otimes 1}\left(q^{\hat{N}} \otimes q^{\hat{N} / 2} b \otimes v n\right)(1 \otimes 1 \otimes v)^{\hat{N} \otimes 1 \otimes 1} \\
& =\left(q^{\hat{N}} \otimes q^{\hat{N} / 2} b \otimes v n\right)(1 \otimes v \otimes 1)^{\hat{N} \otimes 1 \otimes 1}(1 \otimes 1 \otimes v)^{\hat{N} \otimes 1 \otimes 1} .
\end{aligned}
$$

Thus $\overline{\mathrm{F}}$ and $\mathrm{P}$ satisfy 6.33 .

\subsubsection{Quantum az+b group}

The group $a z+b$ with coefficients $a \in \mathbb{C} \backslash\{0\}, b \in \mathbb{C}$ is the group of affine transformations of the complex plane $\mathbb{C}$. Its quantum deformation $\mathbb{I}=\left(C, \Delta_{C}\right)$ in the $\mathrm{C}^{*}$-algebraic framework was introduced in [50] by Woronowicz. For simplicity, we consider the deformation parameter $0<q<1$. A pair of normal operators $(a, b)$ is an $\mathbb{I}$-pain ${ }^{1}$ on a Hilbert space $\mathcal{H}$ if

$$
\begin{aligned}
& \operatorname{Sp}(a) \subset \overline{\mathbb{C}}_{(q)}, \operatorname{ker}(a)=\{0\}, \quad \operatorname{Sp}(b) \subset \overline{\mathbb{C}}_{(q)}, \\
& (\operatorname{Phase}(a)) b(\operatorname{Phase}(a))^{*}=q b, \quad|a|^{-\mathrm{i} t} b|a|^{\mathrm{i} t}=q^{-\mathrm{i} t} b, \quad \text { for all } t \in \mathbb{R} \text {. }
\end{aligned}
$$

In the quantum version, the pair $(a, b)$ in the classical $a z+b$ group is replaced by an I-pair.

For any $\eta, \eta^{\prime} \in \mathbb{C}_{(q)}$ we write $\eta=q^{\mathrm{i} \varphi+k}$ and $\eta^{\prime}=q^{\mathrm{i} \psi+l}$ and let

$$
\chi\left(\eta, \eta^{\prime}\right):=q^{\mathrm{i}(\varphi l+k \psi)} .
$$

Then $\chi$ is a bicharacter on the multiplicative group $\mathbb{C}_{(q)}$.

Given an $\mathbb{I}$-pair $(a, b)$, let $\Lambda=\operatorname{Sp}(a) \times \operatorname{Sp}(b)$, and

$$
\chi\left(b^{-1} \otimes 1,1 \otimes a\right):=\int_{\Lambda} \chi\left(\lambda^{-1}, \lambda^{\prime}\right) \mathrm{d} E_{b}(\lambda) \otimes \mathrm{d} E_{a}\left(\lambda^{\prime}\right),
$$

where $\mathrm{d} E_{a}$ and $\mathrm{d} E_{b}$ are the respective spectral measures on $\operatorname{Sp}(a)$ and $\operatorname{Sp}(b)$.

Next we recall the description of the underlying $\mathrm{C}^{*}$-algebra of quantum $a z+b$. For any $\xi \in \overline{\mathbb{C}}_{(q)}$ set

$$
b(\xi):=\xi .
$$

Then $b \in \mathrm{C}\left(\overline{\mathbb{C}}_{(q)}\right)$ is normal and $\operatorname{Sp}(b) \subset \overline{\mathbb{C}}_{(q)}$. Therefore, $b$ is affiliated to $\mathrm{C}_{0}\left(\overline{\mathbb{C}}_{(q)}\right)$. There is an action of $\mathbb{C}_{(q)}$ on $\mathrm{C}_{0}\left(\overline{\mathbb{C}}_{(q)}\right)$ defined by

$$
\left(\gamma_{\eta} f\right)(\xi):=f(\eta \cdot \xi) \quad \text { for } \eta \in \mathbb{C}_{(q)}, f \in \mathrm{C}_{0}\left(\overline{\mathbb{C}}_{(q)}\right), \xi \in \overline{\mathbb{C}}_{(q)} .
$$

\footnotetext{
1 Originally, in 50, such pairs $(a, b)$ are called $G$-pairs, where $G$ denotes quantum $a z+b$. In order to keep notation consistent we rename them $\mathbb{I}$-pair.
} 
Let $C=\mathrm{C}_{0}\left(\overline{\mathbb{C}}_{(q)}\right) \rtimes \mathbb{C}_{(q)}$ be the corresponding crossed product. Since the canonical embedding $\mathrm{C}_{0}\left(\overline{\mathbb{C}}_{(q)}\right) \hookrightarrow$ $\mathcal{M}(C)$ is nondegenerate, $b$ is affiliated to $C$. By definition, $\mathcal{M}(C)$ contains a strictly continuous one-parameter group of unitaries $\left(\mathrm{U}_{\eta}\right)_{\eta \in \mathbb{C}_{(q)}}$ implementing the action $\gamma$ :

$$
\mathrm{U}_{\eta} f \mathrm{U}_{\eta}^{*}=\gamma_{\eta} f \quad \text { for } f \in \mathrm{C}_{0}\left(\overline{\mathbb{C}}_{(q)}\right) .
$$

It was shown, in [50. Section 4], that $\mathrm{U}_{\eta}=\chi(a, \eta)$, where $a$ is a normal operator affiliated to $C$ and $\operatorname{Sp}(a) \subset$ $\overline{\mathbb{C}}_{(q)}$. Moreover, $a$ is invertible and $a^{-1}$ is also affiliated to $C$.

Given $\eta \in \mathbb{C}_{(q)}, \xi \in \overline{\mathbb{C}}_{(q)}$ we have $\gamma_{\eta}(b)(\xi)=b(\eta \cdot \xi)=\eta \cdot \xi$. Therefore, $\gamma_{\eta} b=\eta \cdot b$, giving $\mathrm{U}_{\eta} b=\eta \cdot b \mathrm{U}_{\eta}$ for $\eta \in \mathbb{C}_{(q)}$. Now $\mathrm{U}_{q}=\operatorname{Phase}(a)$ and $\mathrm{U}_{q^{\mathrm{i} t}}=|a|^{-\mathrm{i} t}$ for $t \in \mathbb{R}$. Let $\pi$ be a nondegenerate representation of $C$ on $\mathcal{H}$. Theorem 1.2 in 47. gives a natural extension of $\pi$ to the elements affiliated to $C$. Thus $(\pi(a), \pi(b))$ is an $\mathbb{I}$-pair on $\mathcal{H}$. Proposition 4.2 in $[50$. shows that every $\mathbb{I}$-pair is of this form.

The comutiplication on $\Delta_{C}: C \rightarrow C \otimes C$ is defined by

$$
\Delta_{C}(a):=a \otimes a, \quad \Delta_{C}(b):=a \otimes b \dot{+} b \otimes 1,
$$

where $\dot{+}$ denotes the closure of the sum. More precisely, $\Delta_{C}(a)$ and $\Delta_{C}(b)$ are affiliated to $C \otimes C$. The spectral condition $\operatorname{Sp}(a), \operatorname{Sp}(b) \subset \overline{\mathbb{C}}_{(q)}$ is crucial to show that $a \otimes b \dot{+} b \otimes 1$ is normal.

A modular multiplicative unitary for quantum $a z+b$ is given by

$$
\mathbb{W}=\mathrm{F}_{q}\left(b^{-1} \otimes a\right) \chi\left(b^{-1} \otimes 1,1 \otimes a\right) .
$$

A corresponding manageable multiplicative unitary is defined by the following theorem due to Woronowicz:

Theorem 6.13 ([50, Theorem A.1]). Let $(a, b)$ be a I-pair, $s$ be a unitary, and $r$ be a strictly positive self-adjoint operator on a Hilbert space $\mathcal{H}$. Assume that $\operatorname{ker}(b)=\{0\}, r$ and s strongly commute with a and $b, \operatorname{Sp}(r) \subseteq q^{\mathbb{Z}} \cup\{0\}$ and $r^{-\mathrm{i} t} s r^{\mathrm{i} t}=q^{-\mathrm{i} t}$ s for $t \in \mathbb{R}$. Then the unitary

$$
\mathbb{W}:=\mathrm{F}_{q}\left(b^{-1} a \otimes b\right) \chi\left(s b^{-1} \otimes 1,1 \otimes a\right) \quad \text { in } \mathcal{U}(\mathcal{H} \otimes \mathcal{H})
$$

is a manageable multiplicative unitary for the quantum $a z+b$ group.

\section{Projection on quantum az $+b$}

In the case of quantum $a z+b$, the Hopf *-homomorphism $f: C \rightarrow C$ defined by $f(a):=a$ and $f(b)=0$ is a projection on quantum $a z+b$. Define the unitary $\mathrm{P}:=\chi\left(s b^{-1} \otimes 1,1 \otimes a\right)$, where $a, b, s$ are as in Theorem 6.13. By (2.2) in 50 we know

$$
\chi\left(s b^{-1}, \eta\right) a \chi\left(s b^{-1}, \eta\right)^{*}=\eta a \quad \text { for all } \eta \in \mathbb{C}_{(q)} .
$$

Therefore, we get

$$
\begin{aligned}
& \chi\left(1 \otimes s b^{-1} \otimes 1,1 \otimes 1 \otimes a\right) \chi\left(s b^{-1} \otimes 1 \otimes 1,1 \otimes a \otimes 1\right) \chi\left(1 \otimes s b^{-1} \otimes 1,1 \otimes 1 \otimes a\right)^{*} \\
& =\chi\left(s b^{-1} \otimes 1 \otimes 1,1 \otimes a \otimes a\right)=\chi\left(s b^{-1} \otimes 1 \otimes 1,1 \otimes a \otimes 1\right) \chi\left(s b^{-1} \otimes 1 \otimes 1,1 \otimes 1 \otimes a\right) .
\end{aligned}
$$

Hence, $\mathrm{P}$ is the projection bicharacter induced by $f$. The corresponding right quantum group homomorphism is defined by $\Delta_{R}:=\left(\operatorname{id}_{C} \otimes f\right) \Delta_{C}$. The comultiplication formula (6.48) shows that $\Delta_{R}(b)=b \otimes 1$ and, clearly, the second leg of the unitary $\overline{\mathrm{F}}:=\mathrm{F}_{q}\left(b^{-1} a \otimes b\right)$ is $\Delta_{R}$-invariant. By the definition $(3.16)$ of $\Delta_{R}$ we get (6.34). The defining conditions of I-pairs (6.47) show that $a \otimes a$ and $b^{-1} a \otimes b$ strongly commute; hence $\mathrm{F}_{q}\left(b^{-1} a \otimes a\right)$ and $a \otimes a$ commute. Therefore,

$$
\chi\left(s b^{-1} \otimes 1 \otimes 1,1 \otimes a \otimes a\right) \mathrm{F}_{q}\left(1 \otimes b^{-1} a \otimes b\right)=\mathrm{F}_{q}\left(1 \otimes b^{-1} a \otimes b\right) \chi\left(s b^{-1} \otimes 1 \otimes 1,1 \otimes a \otimes a\right)
$$

shows that $\overline{\mathrm{F}}$ and $\mathrm{P}$ satisfy 6.33 . 


\subsubsection{Quantum ax +b group}

The group $a x+b$ with coefficients $a \in \mathbb{R}_{>0}, b \in \mathbb{R}$ is the group of orientation-preserving affine transformations of the real line $\mathbb{R}$. Its quantum deformation $\mathbb{I}=\left(C, \Delta_{C}\right)$ in the $\mathrm{C}^{*}$-algebraic framework was introduced by Woronowicz and Zakrzewski in 55. The deformation parameter $q \in \mathbb{T}$ is such that $q^{2}=e^{-\mathrm{i} \hbar}$, where $\hbar$ is a real number such that $0<\hbar<\pi$. The current construction is more delicate compared to quantum $a z+b$.

Let $a$ and $b$ be self-adjoint operators on a Hilbert space $\mathcal{H}$. Assume that $a$ is strictly positive. We write $a \multimap b$ if

$$
a^{i t} b a^{-i t}=e^{\hbar t} b \quad \text { for all } t \in \mathbb{R} .
$$

The notion of $\mathbb{I}$-pair (see 6.47), for a given pair $(a, b)$ on a Hilbert space $\mathcal{H}$ becomes as follows:

$a$ is strictly positive and selfadjoint, $\quad b$ is selfadjoint, and $a \multimap b$.

But the comultiplication $\Delta_{C}(b)=a \otimes b+b \otimes 1$ fails to give a self-adjoint operator in general. This is the fundamental difference between quantum $a z+b$ and quantum $a x+b$. To overcome this difficulty the notion of $\mathbb{I}$-triple (originally called $G$-triple) was introduced in 55. Let $a, b, \beta$ be self-adjoint operators acting on a Hilbert space $\mathcal{H}$. A triple $(a, b, \beta)$ is called an $\mathbb{I}$-triple if

$$
(a, b) \text { is an } \mathbb{I} \text {-pair on } \mathcal{H}, \beta^{2}=\delta(b \neq 0), \beta a=a \beta \text { and } \beta b=-b \beta,
$$

where $\delta$ is the characteristic function (not a bicharacter) defined by

$$
\delta(A)= \begin{cases}1 & \text { if } A \text { is true } \\ 0 & \text { if } A \text { is false }\end{cases}
$$

Passing from $\mathbb{I}$-pairs to $\mathbb{I}$-triples adds one extra generator $\beta$. Therefore, the $\mathrm{C}^{*}$-algebra $C$ corresponds to an extended quantum $a x+b$.

Given an $\mathbb{I}$-triple $(a, b, \beta)$ the reflection operator $\tau$ is defined by $\tau=\alpha(\beta \otimes \beta) \delta(b \otimes b<0)$, where $\alpha= \pm 1$. Here $\alpha$ is the phase factor associated to the deformation parameter $\hbar$ related as $\alpha \mathrm{i} e^{\frac{\mathrm{i} \pi^{2}}{2 \hbar}}$. The condition $\alpha= \pm 1$ restricts the values of the deformation parameter to $\hbar=\frac{\pi}{2 k+3}$, where $k=0,1,2 \cdots$.

Now we extend the domain of $a \otimes b+b \otimes 1$ to

$$
\mathcal{D o m}(a \otimes b+b \otimes 1)+\left\{x \in \mathcal{D} \operatorname{om}\left((a \otimes b+b \otimes 1)^{*}\right): \tau(x)=x\right\} .
$$

Then $[a \otimes b+b \otimes 1]_{\tau}$ is a self-adjoint operator defined as the restriction of $(a \otimes b+b \otimes 1)^{*}$ to the extended domain above.

Let $B=\mathrm{C}_{0}([0,+\infty)) \otimes \mathbb{M}_{2}(\mathbb{C})$ and $B_{0}=\mathrm{C}_{0}((0,+\infty)) \otimes \mathbb{M}_{2}(\mathbb{C})$. The underlying $\mathrm{C}^{*}$-algebra $C$ of the quantum $a x+b$ group is defined by $C=B_{0} \rtimes \mathbb{R}$, where $\mathbb{R}$ acts by

$$
\left(\gamma_{t} f\right)(s):=f\left(e^{\hbar t} s\right) \quad \text { for } t, s \in \mathbb{R}, f \in B_{0} .
$$

The operators $\log (a), b, \mathrm{i} \beta$ are affiliated to $C$ (for details see [55, Section 3]), and [55, Proposition 3.1] shows that $C$ is generated by $\log (a), b, \mathrm{i} \beta$. Then the comultiplication $\Delta_{C}: C \rightarrow C \otimes C$ is defined by

$$
\begin{aligned}
\Delta_{C}(a) & :=a \otimes a, \quad \Delta_{C}(b):=[a \otimes b+b \otimes 1]_{\tau}, \\
\Delta_{C}(i \beta b) & :=\mathrm{i}\left\{w\left(e^{\frac{\mathrm{i} \hbar}{2}} b^{-1} a \otimes b\right)^{-1}(\beta \otimes 1)+(1 \otimes \beta) w\left(e^{\frac{\mathrm{i} \hbar}{2}} b^{-1} a \otimes b^{-1}\right)^{-1}\right\} \Delta_{C}(b),
\end{aligned}
$$

where $w$ is the polynomial of order $2 k+3$ defined by

$$
w(t):=\prod_{l=1}^{2 k+3}\left(1+e^{\mathrm{i}\left(\frac{1}{2}-l\right) \hbar} t\right) \quad \text { for all } t \in \mathbb{R} .
$$

Theorem 6.14. Let $(a, b, \beta)$ be an I-triple and let $r, s$ be strictly positive self-adjoint operators on a Hilbert space $\mathcal{H}$. Assume that $\operatorname{ker}(b)=\{0\}, r$ and $s$ strongly commute with $a, b$ and $\beta$ and $r \multimap s$. Then the unitary defined by

$$
\mathbb{W}:=\mathrm{F}_{\hbar}\left(e^{\frac{\mathrm{i} \hbar}{2}} b^{-1} a \otimes b, \tau\right)^{*} e^{\frac{i}{\hbar} \log \left(s|b|^{-1}\right) \otimes \log (a)} \quad \text { in } \mathcal{U}(\mathcal{H} \otimes \mathcal{H})
$$

is a manageable multiplicative unitary for the quantum $a x+b$ group. 


\section{Projection on quantum $a x+b$}

The projection on (extended) quantum $a x+b$ is induced by the Hopf ${ }^{*}$-homomorphism $f: C \rightarrow C$ defined by $f(a):=a, f(b):=0$; hence by the definition of I-triples 6.50 $f(\beta)=0$. Moreover, $\left(\operatorname{id}_{\mathcal{H}} \otimes f\right) \tau=$ $\left(\operatorname{id}_{\mathcal{H}} \otimes f\right) \alpha(\beta \otimes \beta) \chi(b \otimes b<0)=0$. Define the unitary $\mathrm{P}$, associated to the projection bicharacter induced by the projection $f$, by

$$
\mathrm{P}:=(\mathrm{id} \otimes f)\left(\mathrm{F}_{\hbar}\left(e^{\frac{\mathrm{i} \hbar}{2}} b^{-1} a \otimes b, \tau\right)^{*} e^{\frac{i}{\hbar} \log \left(s|b|^{-1}\right) \otimes \log (a)}\right)=e^{\frac{i}{\hbar} \log \left(s|b|^{-1}\right) \otimes \log (a)} .
$$

By definition, $w(0)=1$. Define the right quantum group homomorphism $\Delta_{R}:=\left(\operatorname{id}_{C} \otimes f\right) \Delta_{C}$. The comultiplication formula shows that $\Delta_{R}(a)=a \otimes a, \Delta_{R}(b)=b \otimes 1$ and $\Delta_{R}(\mathrm{i} \beta b)=\mathrm{i} \beta b \otimes 1$. Therefore, the second leg of the unitary $\overline{\mathrm{F}}:=\mathrm{F}_{\hbar}\left(e^{\frac{\mathrm{i} \hbar}{2}} b^{-1} a \otimes b, \tau\right)^{*}$ is $\Delta_{R}$-invariant. Clearly,

$$
\mathrm{P}_{12} \mathrm{P}_{13}=e^{\frac{\mathrm{i}}{2} \log \left(s|b|^{-1}\right) \otimes(\log (a) \otimes 1+1 \otimes \log (a))} .
$$

Since $a \multimap b$, we get $a^{\mathrm{it}} b a^{-\mathrm{i} t}=e^{\hbar t} b$ for all $t \in \mathbb{R}$. Putting $t=-\mathrm{i}$ yields strong commutation of $e^{\log (a) \otimes 1+1 \otimes \log (a)}$ and $e^{\frac{i}{2}} b^{-1} a \otimes b$. The defining conditions of $\mathbb{I}$-triples show that $e^{\log (a) \otimes 1+1 \otimes \log (a)}$ and $\tau$ commute. Therefore,

$$
\mathrm{P}_{12} \mathrm{P}_{13} \mathrm{~F}_{\hbar}\left(1 \otimes e^{\frac{\mathrm{i} \hbar}{2}} b^{-1} a \otimes b, \tau\right)^{*}=\mathrm{F}_{\hbar}\left(1 \otimes e^{\frac{\mathrm{i} \hbar}{2}} b^{-1} a \otimes b, \tau\right)^{*} \mathrm{P}_{12} \mathrm{P}_{13}
$$

Similarly, 3.16 shows that $\mathrm{P}_{23} \overline{\mathrm{F}}_{12}=\overline{\mathrm{F}}_{12} \mathrm{P}_{23}$. Thus $\mathrm{P}$ and $\overline{\mathrm{F}}$ satisfy 6.33 and 6.34.

\subsection{Construction of a standard multiplicative unitary}

A braided multiplicative unitary $\mathbb{F} \in \mathcal{U}(\mathcal{L} \otimes \mathcal{L})$ over $\mathbb{G}$ yields a multiplicative unitary in the usual sense. Thus we can construct a "big" quantum group starting from a standard and a braided quantum group, which is our semidirect product for $\mathrm{C}^{*}$-quantum group.

Theorem 6.15. The unitary $\mathbb{W}_{1234} \in \mathcal{U}(\mathcal{H} \otimes \mathcal{L} \otimes \mathcal{H} \otimes \mathcal{L})$ defined by

$$
\mathbb{W}_{1234}:=\mathbb{W}_{13}^{A} \mathbb{U}_{23} \hat{\mathbb{V}}_{34}^{*} \mathbb{F}_{24} \hat{\mathbb{V}}_{34}
$$

is a multiplicative unitary.

Proof. We need to verify 2.4 for $\mathbb{W}_{1234}$. Since it is going to be a long routine computation we write it step by step in order to avoid confusion. Whenever we use relation(s) to a group of terms to simplify we enclose them by $\{\cdots\}$.

Let L.H.S. $=\mathbb{W}_{3456} \mathbb{W}_{1234} \mathbb{W}_{3456}^{*}$. Using 6.55 we write:

$$
\text { L.H.S. }=\mathbb{W}_{35}^{A}\left\{\mathbb{U}_{45} \hat{\mathbb{V}}_{56}^{*} \mathbb{F}_{46} \hat{\mathbb{V}}_{56}\right\}\left\{\mathbb{W}_{13}^{A} \mathbb{U}_{23}\right\} \hat{\mathbb{V}}_{34}^{*} \mathbb{F}_{24} \hat{\mathbb{V}}_{34} \hat{\mathbb{V}}_{56}^{*} \mathbb{F}_{46}^{*} \hat{\mathbb{V}}_{56} \mathbb{U}_{45}^{*}\left(\mathbb{W}_{35}^{A}\right)^{*} .
$$

Now $\mathbb{U}_{45} \hat{\mathbb{V}}_{56}^{*} \mathbb{F}_{46} \hat{\mathbb{V}}_{56}$ and $\mathbb{W}_{13}^{A} \mathbb{U}_{23}$ commute,

$$
\text { L.H.S. }=\left\{\mathbb{W}_{35}^{A} \mathbb{W}_{13}^{A}\right\} \mathbb{U}_{23} \mathbb{U}_{45} \hat{\mathbb{V}}_{56}^{*} \mathbb{F}_{46}\left\{\hat{\mathbb{V}}_{56}\right\} \hat{\mathbb{V}}_{34}^{*} \mathbb{F}_{24} \hat{\mathbb{V}}_{34}\left\{\hat{\mathbb{V}}_{56}^{*}\right\} \mathbb{F}_{46}^{*} \hat{\mathbb{V}}_{56} \mathbb{U}_{45}^{*}\left(\mathbb{W}_{35}^{A}\right)^{*} .
$$

Using 2.4 and the ordinary commutation between $\hat{\mathbb{V}}_{56}, \hat{\mathbb{V}}_{34}^{*} \mathbb{F}_{24} \hat{\mathbb{V}}_{34}$, we get

$$
\text { L.H.S. }=\mathbb{W}_{13}^{A} \mathbb{W}_{15}^{A}\left\{\mathbb{W}_{35}^{A} \mathbb{U}_{23}\right\} \mathbb{U}_{45} \hat{\mathbb{V}}_{56}^{*}\left\{\mathbb{F}_{46} \hat{\mathbb{V}}_{34}^{*}\right\} \mathbb{F}_{24}\left\{\hat{\mathbb{V}}_{34} \mathbb{F}_{46}^{*}\right\} \hat{\mathbb{V}}_{56} \mathbb{U}_{45}^{*}\left(\mathbb{W}_{35}^{A}\right)^{*} .
$$

Equations 6.4, 6.8 and then the ordinary commutations between $\hat{\mathbb{V}}_{56}$ and $\hat{\mathbb{V}}_{34}, \hat{\mathbb{V}}_{36}$ and $\mathbb{F}_{24}$ yield

$$
\begin{aligned}
\text { L.H.S. } & =\mathbb{W}_{13}^{A} \mathbb{W}_{15}^{A} \mathbb{U}_{23} \mathbb{U}_{25} \mathbb{W}_{35}^{A} \mathbb{U}_{45}\left\{\hat{\mathbb{V}}_{56}^{*} \hat{\mathbb{V}}_{34}^{*}\right\} \hat{\mathbb{V}}_{36}^{*} \mathbb{F}_{46}\left\{\hat{\mathbb{V}}_{36}\right\} \mathbb{F}_{24}\left\{\hat{\mathbb{V}}_{36}^{*}\right\} \mathbb{F}_{46}^{*} \hat{\mathbb{V}}_{36}\left\{\hat{\mathbb{V}}_{34} \hat{\mathbb{V}}_{56}\right\} \mathbb{U}_{45}^{*}\left(\mathbb{W}_{35}^{A}\right)^{*} \\
& =\mathbb{W}_{13}^{A} \mathbb{W}_{15}^{A} \mathbb{U}_{23} \mathbb{U}_{25}\left\{\mathbb{W}_{35}^{A} \mathbb{U}_{45} \hat{\mathbb{V}}_{34}^{*}\right\} \hat{\mathbb{V}}_{56}^{*} \hat{\mathbb{V}}_{36}^{*} \mathbb{F}_{46} \mathbb{F}_{24} \mathbb{F}_{46}^{*} \hat{\mathbb{V}}_{36} \hat{\mathbb{V}}_{56}\left\{\hat{\mathbb{V}}_{34} \mathbb{U}_{45}^{*}\left(\mathbb{W}_{35}^{A}\right)^{*}\right\} .
\end{aligned}
$$


The braided pentagon equation $(6.6)$, equation $(6.9)$ and then commutation between $\mathbb{W}_{35}^{A}$ and $\mathbb{F}_{24} Z_{46} \mathbb{F}_{26} Z_{46}^{*}$ yield

$$
\begin{aligned}
\text { L.H.S. } & =\mathbb{W}_{13}^{A} \mathbb{W}_{15}^{A} \mathbb{U}_{23} \mathbb{U}_{25} \hat{\mathbb{V}}_{34}^{*} \mathbb{U}_{45} \hat{\mathbb{V}}_{56}^{*}\left\{\mathbb{W}_{35}^{A}\right\}\left\{\mathbb{F}_{24} Z_{46} \mathbb{F}_{26} Z_{46}^{*}\right\}\left\{\left(\mathbb{W}^{A}\right)_{35}^{*}\right\} \hat{\mathbb{V}}_{56} \mathbb{U}_{45}^{*} \hat{\mathbb{V}}_{34} \\
& =\mathbb{W}_{13}^{A} \mathbb{W}_{15}^{A} \mathbb{U}_{23}\left\{\mathbb{U}_{25} \hat{\mathbb{V}}_{34}^{*}\right\} \mathbb{U}_{45}\left\{\hat{\mathbb{V}}_{56}^{*} \mathbb{F}_{24}\right\} Z_{46} \mathbb{F}_{26}\left\{Z_{46}^{*} \hat{\mathbb{V}}_{56} \mathbb{U}_{45}^{*}\right\} \hat{\mathbb{V}}_{34} .
\end{aligned}
$$

Using commutation between $\mathbb{U}_{25}$ and $\hat{\mathbb{V}}_{34}, \hat{\mathbb{V}}_{56}$ and $\mathbb{F}_{24}$ and then using 6.2 we get:

$$
\text { L.H.S. }=\mathbb{W}_{13}^{A} \mathbb{W}_{15}^{A} \mathbb{U}_{23} \hat{\mathbb{V}}_{34}^{*}\left\{\mathbb{U}_{25} \mathbb{U}_{45} \mathbb{F}_{24} \mathbb{U}_{45}^{*}\right\} \hat{\mathbb{V}}_{56}^{*}\left\{\mathbb{U}_{45} \mathbb{F}_{26} \mathbb{U}_{45}^{*}\right\} \hat{\mathbb{V}}_{56} \hat{\mathbb{V}}_{34} .
$$

Next we use 6.3 and commutation between $\mathbb{F}_{26}$ and $\mathbb{U}_{45}$ to get the following equality

$$
\text { L.H.S. }=\mathbb{W}_{13}^{A}\left\{\mathbb{W}_{15}^{A}\right\}\left\{\mathbb{U}_{23} \hat{\mathbb{V}}_{34}^{*} \mathbb{F}_{24}\right\}\left\{\mathbb{U}_{25} \hat{\mathbb{V}}_{56}^{*} \mathbb{F}_{26} \hat{\mathbb{V}}_{56}\right\}\left\{\hat{\mathbb{V}}_{34}\right\} .
$$

Finally, using commutation between $\mathbb{W}_{15}^{A}$ and $\mathbb{U}_{23} \hat{\mathbb{V}}_{34}^{*} \mathbb{F}_{24}, \mathbb{U}_{25} \hat{\mathbb{V}}_{56}^{*} \mathbb{F}_{26} \hat{\mathbb{V}}_{56}$ and $\hat{\mathbb{V}}_{34}$ we obtain the desired pentagon equalion 2.4 for $\mathbb{W}_{1234}$ :

$$
\text { L.H.S. }=\left\{\mathbb{W}_{13}^{A} \mathbb{U}_{23} \hat{\mathbb{V}}_{34}^{*} \mathbb{F}_{24} \hat{\mathbb{V}}_{34}\right\}\left\{\mathbb{W}_{15}^{A} \mathbb{U}_{25} \hat{\mathbb{V}}_{56}^{*} \mathbb{F}_{26} \hat{\mathbb{V}}_{56}\right\}=\mathbb{W}_{1234} \mathbb{W}_{1256}
$$

Next we show that $\mathbb{W}_{1234}$ is manageable.

Theorem 6.16. If $\mathbb{F} \in \mathcal{U}(\mathcal{L} \otimes \mathcal{L})$ is manageable, so is $\mathbb{W}_{1234}:=\mathbb{W}_{13}^{A} \mathbb{U}_{23} \hat{\mathbb{V}}_{34}^{*} \mathbb{F}_{24} \hat{\mathbb{V}}_{34}$.

Proof. Using 6.2$)$ we can rewrite 6.55$)$ in the following way:

$$
\mathbb{W}_{1234}=\mathbb{W}_{13}^{A} \hat{\mathbb{V}}_{34}^{*} \mathbb{U}_{23} Z_{24}^{*} \mathbb{F}_{24} \hat{\mathbb{V}}_{34} .
$$

Definition 6.3 and Lemma 6.4 show that $\mathbb{W}_{1234}$ commutes with $Q_{A} \otimes Q_{K} \otimes Q_{A} \otimes Q_{K}$.

We shall show that

$$
\widetilde{\mathbb{W}}_{1234}:=\widetilde{\mathbb{W}}_{13}^{A} \hat{\mathbb{V}}_{34}^{*} \widetilde{\mathbb{F}}_{24} \widetilde{\mathbb{U}}_{23} \hat{\mathbb{V}}_{23} \in \mathcal{U}(\overline{\mathcal{H}} \otimes \overline{\mathcal{L}} \otimes \mathcal{H} \otimes \mathcal{L})
$$

satisfies the manageability condition $\left[\left(\right.\right.$ ii) for $\mathbb{W}_{1234}$.

Let $x, y \in \mathcal{L}, s \in \mathcal{D} \operatorname{om}\left(Q_{A}\right), t \in \mathcal{D} \operatorname{Dom}\left(Q_{K}\right), u \in \mathcal{D} \operatorname{om}\left(Q_{A}^{-1}\right)$ and $v \in \mathcal{D} \operatorname{Dom}\left(Q_{B}^{-1}\right)$. Let $\left\{e_{i}\right\}_{i=1,2, \cdots}$ be an orthonormal basis of $\mathcal{L}$. Then 3.12 and Definition 6.3 yield:

$$
\begin{aligned}
\left(x \otimes s \otimes t\left|\mathbb{U}_{12} Z_{13}^{*} \mathbb{F}_{13}\right| y \otimes u \otimes v\right) & =\sum_{i}\left(x \otimes s \otimes t\left|\mathbb{U}_{12} \cdot\left(\mid e_{i}\right) \cdot\left(e_{i} \mid \otimes 1 \otimes 1\right) \cdot Z_{13}^{*} \mathbb{F}_{13}\right| y \otimes u \otimes v\right) \\
& =\sum_{i}\left(x \otimes s|\mathbb{U}| e_{i} \otimes u\right) \cdot\left(e_{i} \otimes t\left|\left(Z^{*} \mathbb{F}\right)\right| y \otimes v\right) \\
& =\sum_{i}\left(\bar{y} \otimes Q_{K}(t)|\widetilde{\mathbb{F}}| \overline{e_{i}} \otimes Q_{K}^{-1}(v)\right)\left(\overline{e_{i}} \otimes Q_{A}(s)|\widetilde{\mathbb{U}}| \bar{x} \otimes Q_{K}^{-1}(u)\right) \\
& =\left(\bar{y} \otimes Q_{A}(s) \otimes Q_{K}(t)\left|\widetilde{\mathbb{F}}_{13} \widetilde{\mathbb{U}}_{12}\right| \bar{x} \otimes Q_{A}^{-1}(u) \otimes Q_{K}^{-1}(v)\right)
\end{aligned}
$$

Since $\hat{\mathbb{V}}$ commutes with $Q_{A} \otimes Q_{K}, \hat{\mathbb{V}}\left(\mathcal{D} \operatorname{om}\left(Q_{A}\right) \otimes \mathcal{D} \operatorname{om}\left(Q_{K}\right)\right)$ and $\hat{\mathbb{V}}\left(\mathcal{D} \operatorname{om}\left(Q_{A}^{-1}\right) \otimes \mathcal{D}\right.$ om $\left.\left(Q_{B}^{-1}\right)\right)$ are dense subspaces of $\mathcal{D} \operatorname{om}\left(Q_{A}\right) \otimes \mathcal{D} \operatorname{om}\left(Q_{K}\right)$ and $\mathcal{D} \operatorname{om}\left(Q_{A}^{-1}\right) \otimes \mathcal{D} \operatorname{om}\left(Q_{B}^{-1}\right)$. Hence we can replace $s \otimes t$ by $\hat{\mathbb{V}}(s \otimes t)$ and $u \otimes v$ by $\hat{\mathbb{V}}(u \otimes v)$ in the last computation. This gives

$$
\begin{aligned}
& \left(x \otimes s \otimes t\left|\hat{\mathbb{V}}_{23}^{*} \mathbb{U}_{12} Z_{13}^{*} \mathbb{F}_{13} \hat{\mathbb{V}}_{23}\right| y \otimes u \otimes v\right) \\
& =\left(\bar{y} \otimes Q_{A}(s) \otimes Q_{K}(t)\left|\hat{\mathbb{V}}_{23}^{*} \widetilde{\mathbb{F}}_{13} \widetilde{\mathbb{U}}_{12} \hat{\mathbb{V}}_{23}\right| \bar{x} \otimes Q_{A}^{-1}(u) \otimes Q_{K}^{-1}(v)\right) .
\end{aligned}
$$

Let $z, w \in \mathcal{H}$ and $\left\{e_{j}\right\}_{j=1,2, \ldots}$ be an orthonormal basis in $\mathcal{H}$ as in Lemma 2.37

$$
\left.\sum_{j} \mid Q_{A}^{-1}\left(e_{j}\right)\right) \cdot\left(Q_{A}\left(e_{j}\right) \mid=1_{\mathcal{H}}\right.
$$


Then the following computation completes the proof:

$$
\begin{aligned}
& \left(z \otimes x \otimes s \otimes t\left|\mathbb{W}_{13}^{A} \hat{\mathbb{V}}_{34}^{*} \mathbb{U}_{23} Z_{24}^{*} \mathbb{F}_{24} \hat{\mathbb{V}}_{34}\right| w \otimes y \otimes u \otimes v\right) \\
& =\sum_{j}\left(z \otimes x \otimes s \otimes t\left|\mathbb{W}_{13}^{A} \cdot\left(1 \otimes 1 \otimes \mid e_{j}\right) \cdot\left(e_{i} \mid \otimes 1\right) \cdot \hat{\mathbb{V}}_{34}^{*} \mathbb{U}_{23} Z_{24}^{*} \mathbb{F}_{24} \hat{\mathbb{V}}_{34}\right| w \otimes y \otimes u \otimes v\right) \\
& =\sum_{j}\left(z \otimes s\left|\mathbb{W}^{A}\right| w \otimes e_{i}\right) \cdot\left(x \otimes e_{i} \otimes t\left|\hat{\mathbb{V}}_{23}^{*} \mathbb{U}_{12}\left(Z^{*} \mathbb{F}\right)_{13} \hat{\mathbb{V}}_{23}\right| y \otimes u \otimes v\right) \\
& =\sum_{j}\left(\bar{w} \otimes Q_{A}(s)\left|\widetilde{\mathbb{W}}^{A}\right| \bar{z} \otimes Q_{A}^{-1}\left(e_{i}\right)\right) \\
& \quad \cdot\left(\bar{y} \otimes Q_{A}\left(e_{i}\right) \otimes Q_{K}(t)\left|\hat{\mathbb{V}}_{23}^{*} \widetilde{\mathbb{F}}_{13} \widetilde{\mathbb{U}}_{12} \hat{\mathbb{V}}_{23}\right| \bar{x} \otimes Q_{A}^{-1}(u) \otimes Q_{K}^{-1}(v)\right) \\
& =\left(\bar{w} \otimes \bar{y} \otimes Q_{A}(s) \otimes Q_{K}(t)\left|\widetilde{\mathbb{W}}_{13}^{A} \hat{\mathbb{V}}_{34}^{*} \widetilde{\mathbb{F}}_{24} \widetilde{\mathbb{U}}_{23} \hat{\mathbb{V}}_{34}\right| \bar{z} \otimes \bar{x} \otimes Q_{A}^{-1}(u) \otimes Q_{K}^{-1}(v)\right) .
\end{aligned}
$$

Let $\mathbb{I}=\left(C, \Delta_{C}\right)$ be the $\mathrm{C}^{*}$-quantum group corresponding to the manageable multiplicative unitary $\mathbb{W}_{1234}$ in Theorem 6.16.

Proposition 6.17. I is a $\mathrm{C}^{*}$-quantum group with projection.

Proof. Let $\mathbb{X}_{1234}=\mathbb{W}_{13}^{A} \mathbb{U}_{23} \in \mathcal{U}(\mathcal{H} \otimes \mathcal{L} \otimes \mathcal{H} \otimes \mathcal{L})$. Assume $\mathbb{X}_{1234}$ is a bicharacter from $\mathbb{I}$ to $\mathbb{I}$. Proposition 2.36 shows that $\mathbb{X}_{1234}=\mathbb{W}_{13}^{A} \mathbb{U}_{23} \in \mathcal{U}(\mathcal{H} \otimes \mathcal{L})$ is a manageable multiplicative unitary for $\mathbb{G}$; hence $X_{1234}$ is a projection. Therefore we need to show that $\mathbb{X}_{1234}$ is a bicharacter.

The following routine computation yields $\sqrt{3.3}$ for $\mathbb{X}_{1234}$ and $\mathbb{W}_{1234}$ :

$$
\begin{aligned}
\mathbb{X}_{3456} \mathbb{W}_{1234} \mathbb{X}_{3456}^{*}=\mathbb{W}_{35}^{A} \mathbb{U}_{45} \mathbb{W}_{13}^{A} \mathbb{U}_{23} \hat{\mathbb{V}}_{34}^{*} \mathbb{F}_{24} \hat{\mathbb{V}}_{34} \mathbb{U}_{45}^{*}\left(\mathbb{W}^{A}\right)_{35}^{*} & =\mathbb{W}_{35}^{A} \mathbb{W}_{13}^{A} \mathbb{U}_{23} \mathbb{U}_{45} \hat{\mathbb{V}}_{34}^{*} \mathbb{F}_{24} \hat{\mathbb{V}}_{34} \mathbb{U}_{45}^{*}\left(\mathbb{W}^{A}\right)_{35}^{*} \\
& =\mathbb{W}_{13}^{A} \mathbb{W}_{15}^{A} \mathbb{W}_{35}^{A} \mathbb{U}_{23} \mathbb{U}_{45} \hat{\mathbb{V}}_{34}^{*} \mathbb{F}_{24} \hat{\mathbb{V}}_{34} \mathbb{U}_{45}^{*}\left(\mathbb{W}^{A}\right)_{35}^{*} \\
& =\mathbb{W}_{13}^{A} \mathbb{W}_{15}^{A} \mathbb{U}_{23} \mathbb{U}_{25} \mathbb{W}_{35}^{A} \mathbb{U}_{45} \hat{\mathbb{V}}_{34}^{*} \mathbb{F}_{24} \hat{\mathbb{V}}_{34} \mathbb{U}_{45}^{*}\left(\mathbb{W}^{A}\right)_{35}^{*} \\
& =\mathbb{W}_{13}^{A} \mathbb{U}_{23} \mathbb{W}_{15}^{A} \mathbb{U}_{25} \hat{\mathbb{V}}_{34}^{*} \mathbb{U}_{45} \mathbb{F}_{24} \mathbb{U}_{45}^{*} \hat{\mathbb{V}}_{34} \\
& =\mathbb{W}_{13}^{A} \mathbb{U}_{23} \hat{\mathbb{V}}_{34}^{*} \mathbb{W}_{15}^{A} \mathbb{U}_{25} \mathbb{U}_{45} \mathbb{F}_{24} \mathbb{U}_{45}^{*} \hat{\mathbb{V}}_{34} \\
& =\mathbb{W}_{13}^{A} \mathbb{U}_{23} \hat{\mathbb{V}}_{34}^{*} \mathbb{W}_{15}^{A} \mathbb{F}_{24} \mathbb{U}_{25} \hat{\mathbb{V}}_{34} \\
& =\mathbb{W}_{13}^{A} \mathbb{U}_{23} \hat{\mathbb{V}}_{34}^{*} \mathbb{F}_{24} \hat{\mathbb{V}}_{34} \mathbb{W}_{15}^{A} \mathbb{U}_{25}=\mathbb{W}_{1234} \mathbb{X}_{1256} .
\end{aligned}
$$

The first equality follows from the assumption, the second equality uses commutation of unitaries, the third equality uses the pentagon equation $(2.4)$ for $\mathbb{W}^{A}$, the fourth equality uses $(2.26)$, the fifth equality follows from 6.1), the sixth equality uses commutation of unitaries, the seventh equality follows from the $\mathbb{G}$-invariance condition 6.3 for $\mathbb{F}$, the eighth equality follows from the commutation of unitaries, and the last equality is trivial.

Similarly, we can show $\mathbb{W}_{3456} \mathbb{X}_{1234}=\mathbb{X}_{1234} \mathbb{X}_{1256} \mathbb{W}_{3456}$ which gives $(3.4)$. Hence, $\mathbb{X}_{1234} \in \mathcal{U}(\mathcal{H} \otimes \mathcal{L} \otimes$ $\mathcal{H} \otimes \mathcal{L})$ is a projection on $\mathbb{I}$.

At first sight, the choice for the expression $\mathbb{W}_{1234}$ in the above Theorem 6.15 seems ad hoc. Let us justify the origin of this formula.

Let $\mathbb{G}$ be a weakly regular quantum group and let $\left(K, \Delta_{K}\right)$ be a braided quantum group over $\mathbb{G}$ (see Definition 6.5). Let $\lambda: K \rightarrow K \otimes A$ be the inner coaction of $\mathbb{G}$ on $K$ defined by $\lambda(k):=\mathbb{U}\left(k \otimes 1_{\hat{A}}\right) \mathbb{U}^{*}$ for $k \in K$.

It can be show that $A \otimes_{\mathrm{W}^{A}} K=\iota_{A}(A) \cdot \iota_{K}(K)$ where $\iota_{A}(a)=\pi(a) \otimes 1_{\mathcal{H}}$ and $\iota_{K}(k)=\hat{\mathbb{V}}^{*}(1 \otimes k) \hat{\mathbb{V}}$, where $\hat{\mathbb{V}}:=\left(\hat{\eta} \otimes \operatorname{id}_{\mathcal{L}}\right) \hat{\mathrm{V}}$ for any $\mathbb{G}$-Heisenberg pair $(\eta, \hat{\eta})$.

Next, by Corollary 5.42 we get the following: $A \otimes_{\mathrm{W}^{A}} K \otimes_{\mathrm{W}^{A}} A \bigotimes_{\mathrm{W}^{A}} K \cong A \bigotimes_{\mathrm{W}^{A}} K \otimes A \bigotimes_{\mathrm{W}^{A}} K$. Denote the embeddings of $A, K, A, K$ into $A \otimes_{\mathrm{W}^{A}} K \otimes A \otimes_{\mathrm{W}^{A}} K$ by $j_{1}, j_{2}, j_{3}, j_{4}$, respectively. Then

$$
\begin{array}{ll}
j_{1}(a)=\left(\iota_{A} \otimes \iota_{A}\right) \Delta_{A}(a), & j_{2}(k)=\left(\iota_{K} \otimes \iota_{A}\right) \lambda(k), \\
j_{3}(a)=\left(1_{A \bigotimes_{\mathrm{W} A} K} \otimes \iota_{A}(a)\right), & j_{4}(k)=\left(1_{A \bigotimes_{\mathrm{W} A} K} \otimes \iota_{K}(k)\right) .
\end{array}
$$


for $a \in A$ and $k \in K$.

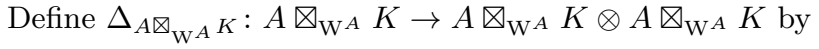

$$
\Delta_{A \varpi_{\mathrm{W} A} K}\left(\iota_{A}(a) \iota_{K}(k)\right):=\left(\iota_{A} \otimes \iota_{A}\right) \Delta_{A}(a) \cdot\left(j_{2} * j_{4}\right) \Delta_{K}(k),
$$

where $j_{2} * j_{4}\left(k_{1} \bigotimes_{\mathrm{W} A} k_{2}\right):=j_{2}\left(k_{1}\right) j_{4}\left(k_{2}\right)$ for $a \in A$ and $k, k_{1}, k_{2} \in K$.

We compute:

$$
\begin{aligned}
\hat{\mathbb{V}}_{12}^{*} \mathbb{U}_{23} \hat{\mathbb{V}}_{34}^{*} \mathbb{F}_{24}=\mathbb{W}_{13}^{A} \mathbb{U}_{23} \hat{\mathbb{V}}_{12}^{*}\left(\mathbb{W}_{13}^{A}\right)^{*} \hat{\mathbb{V}}_{34}^{*} \mathbb{F}_{24} & =\mathbb{W}_{13}^{A} \mathbb{U}_{23} \hat{\mathbb{V}}_{12}^{*} \hat{\mathbb{V}}_{34}^{*} \hat{\mathbb{V}}_{14}^{*}\left(\mathbb{W}_{13}^{A}\right)^{*} \mathbb{F}_{24} \\
& =\mathbb{W}_{13}^{A} \mathbb{U}_{23} \hat{\mathbb{V}}_{34}^{*} \hat{\mathbb{V}}_{12}^{*} \hat{\mathbb{V}}_{14}^{*} \mathbb{F}_{24}\left(\mathbb{W}_{13}^{A}\right)^{*} \\
& =\mathbb{W}_{13}^{A} \mathbb{U}_{23} \hat{\mathbb{V}}_{34}^{*} \mathbb{F}_{24} \hat{\mathbb{V}}_{12}^{*} \hat{\mathbb{V}}_{14}^{*}\left(\mathbb{W}_{13}^{A}\right)^{*}
\end{aligned}
$$

The first equality uses $\sqrt{6.1}$, the second equality follows from the corepresentation condition $(6.9)$, the third equality follows from the ordinary commutations between $\hat{\mathbb{V}}_{34}$ and $\hat{\mathbb{V}}_{12}, \mathbb{W}_{13}^{A}$ and $\mathbb{F}_{24}$, the fourth equality uses the invariance property of $\mathbb{F}$ under the $\widehat{\mathbb{G}}$-coaction 6.4 .

Therefore, using the last relation we obtain:

$$
\begin{aligned}
& \left(\iota_{A} \otimes \iota_{A}\right) \Delta_{A}(a) \cdot\left(j_{2} * j_{4}\right) \Delta_{K}(k) \\
& =\left((\pi \otimes \pi) \Delta_{A}(a)\right)_{13} \hat{\mathbb{V}}_{12}^{*} \mathbb{U}_{23} \hat{\mathbb{V}}_{34}^{*} \mathbb{F}_{24}(k)_{2}\left(\hat{\mathbb{V}}_{12}^{*} \mathbb{U}_{23} \hat{\mathbb{V}}_{34}^{*} \mathbb{F}_{24}\right)^{*} \\
& =\mathbb{W}_{13}^{A} \pi(a)_{1}\left(\mathbb{W}^{A}\right)_{13}^{*} \mathbb{W}_{13}^{A} \mathbb{U}_{23} \hat{\mathbb{V}}_{34}^{*} \mathbb{F}_{24} \hat{\mathbb{V}}_{12}^{*} \hat{\mathbb{V}}_{14}^{*}\left(\mathbb{W}_{13}^{A}\right)^{*}(k)_{2}\left(\mathbb{W}_{13}^{A} \mathbb{U}_{23} \hat{\mathbb{V}}_{34}^{*} \mathbb{F}_{24} \hat{\mathbb{V}}_{12}^{*} \hat{\mathbb{V}}_{14}^{*}\right)^{*} \\
& =\mathbb{W}_{13}^{A} \mathbb{U}_{23} \hat{\mathbb{V}}_{34}^{*} \mathbb{F}_{24} \pi(a)_{1} \hat{\mathbb{V}}_{12} k_{2} \hat{\mathbb{V}}_{12}^{*}\left(\mathbb{W}_{13}^{A} \mathbb{U}_{23} \hat{\mathbb{V}}_{34}^{*} \mathbb{F}_{24}\right)^{*}=\mathbb{W}_{1234}\left(\iota_{A}(a) \cdot \iota_{K}(k) \otimes 1_{A \bigotimes_{\mathrm{W}} K}\right) \mathbb{W}_{1234}^{*} .
\end{aligned}
$$

Hence, the comultiplication on $A \bigotimes_{\mathrm{W}^{A}} K$ is implemented by the manageable multiplicative unitary $\mathbb{W}_{1234}$ defined by 6.55 . Moreover, $\mathbb{W}_{1234}$ is a unitary in $\mathcal{M}\left(\mathcal{H} \otimes \mathcal{L} \otimes A \bigotimes_{\mathrm{W}^{A}} K\right)$. Therefore, we expect the general theory of braided quantum groups to generate $\left(A{\varpi_{\mathrm{W} A}} K, \Delta_{A \unrhd_{\mathrm{W} A} K}\right)$ as a quantum group from the manageable multiplicative unitary $\mathbb{W}_{1234}$.

\subsection{From the complex quantum plane to simplified quantum $\mathbf{E}(2)$}

First we recall the simplified version of quantum E(2) from an unpublished work 54 of Woronowicz. Let

$$
G=\left\{\left(\begin{array}{cc}
u & m \\
0 & 1
\end{array}\right): u, m \in \mathbb{C} \text { and }|u|=1\right\} .
$$

Let $0<q<1$ be a deformation parameter and let $C$ be the ${ }^{*}$-algebra generated by $u$ and $m$ subject to the following relations:

$$
u^{*} u=u u^{*}=1, \quad m^{*} m=q^{2} m m^{*}, \quad \text { and } u m u^{*}=q^{2} m .
$$

The comultiplication $\Delta_{C}: C \rightarrow C \odot C$ is defined by

$$
\Delta_{C}(u):=u \odot u \quad \text { and } \quad \Delta_{C}(m):=u \odot m+m \odot 1 .
$$

where $\odot$ denotes algebraic tensor product. Clearly, $\Delta_{C}$ is coassociative.

Recall $\left(C^{\prime}, \Delta_{C^{\prime}}\right)$ in Section 6.2.2 and the relations 6.39 between the generators $v$ and $n$ of quantum $\mathrm{E}(2)$. A simple computation shows that $\left(v^{2}, v n\right)$ satisfies $\sqrt{6.59}$; hence generates $C$. Define the Hopf ${ }^{*}$-homomorphism $\varphi: C^{\prime} \rightarrow C$ by $\varphi(v):=v^{2}$ and $\varphi(n):=v n$. The kernel of $\varphi$ is isomorphic to $\mathbb{Z} / 2 \mathbb{Z}$. Therefore, quantum $\mathrm{E}(2)$ is the double cover of simplified $\mathrm{E}(2)$.

In order to describe the $\mathrm{C}^{*}$-algebraic version of simplified quantum $\mathrm{E}(2)$ we need to represent $u$ and $m$ on a Hilbert space $\mathcal{H}$. The relations 6.42 between the generators of $\mathrm{E}_{q}(2)$ is replaced by the following:

$$
u^{*} u=u u^{*}=1, \quad m^{*} m=q^{2} m m^{*}, \quad \operatorname{Sp}(m) \subset \overline{\mathbb{C}}_{(q)} \quad \text { and } u m u^{*}=q^{2} m .
$$


Similarly, we replace the operator domains $\mathcal{E}_{\mathcal{H}}$ and $\mathcal{G}_{\mathcal{H}}^{\prime}$ defined by 6.43 and $(6.44)$ by the following operator domains

$$
\begin{aligned}
\mathcal{F}_{\mathcal{H}} & :=\left\{r \in \mathcal{C}(\mathcal{H}): r r^{*}=q^{2} r^{*} r, \operatorname{Sp}(r) \subset \overline{\mathbb{C}}_{(q)}\right\}, \\
\mathcal{G}_{\mathcal{H}} & :=\left\{(u, m) \in \mathcal{U}(\mathcal{H}) \times \mathcal{F}_{\mathcal{H}}: u m u^{*}=q^{2} m\right\} .
\end{aligned}
$$

Therefore, the underlying $\mathrm{C}^{*}$-algebra $C$ of simplified $\mathrm{E}_{q}(2)$ is generated by $(u, m) \in \mathcal{G}_{\mathcal{H}}$. The comultiplication $\Delta_{C}: C \rightarrow C \otimes C$ is defined by

$$
\Delta_{C}(u)=u \otimes u, \quad \Delta_{C}(m)=u \otimes m \dot{+} m \otimes 1,
$$

where $\dot{+}$ denotes the closure of the sum. More precisely, $\Delta_{C}(m)$ is affiliated to $C \otimes C$ and $\operatorname{Sp}(m) \subset \overline{\mathbb{C}}_{(q)}$ plays the crucial role in it.

To find a multiplicative unitary we need to use the quantum exponential function from 52 . First we define the following operator domain:

$$
\widehat{\mathcal{G}_{\mathcal{H}}}:=\left\{(\hat{N}, \hat{m}) \in \mathcal{C}(\mathcal{H}) \times \mathcal{F}_{\mathcal{H}}^{*}: \hat{N}=\hat{N}^{*}, \operatorname{Sp}(\hat{N}) \subset \mathbb{N}, \hat{N} \hat{m}=\hat{m}(\hat{N}+1)\right\} .
$$

The following Theorem classifies all multiplicative unitaries of simplified $\mathrm{E}_{q}(2)$.

Theorem 6.18 ([54]). The unitary

$$
\mathbb{W}:=\mathrm{F}_{q}(\hat{m} \otimes m)(1 \otimes u)^{\hat{N} \otimes 1} \quad \text { in } \mathcal{U}(\mathcal{H} \otimes \mathcal{H})
$$

is a multiplicative unitary for simplified $\mathrm{E}_{q}(2)$ if and only if

1. $(u, m) \in \mathcal{G}_{\mathcal{H}}, \operatorname{Ker}(m)=\{0\}$,

2. $u^{*} \hat{N} u=\hat{N}+1$,

3. $\hat{N}$ and $m$ strongly commute,

4. $\hat{m}=m^{-1} u \dot{+} \hat{r}$ where $\dot{+}$ denotes the closure and $\hat{r} \in \mathcal{C}(\mathcal{H})$ is such that

$$
(\hat{N}, \hat{r}) \in \widehat{\mathcal{G}}_{\mathcal{H}}, \quad u^{*} \hat{r} u=q^{2} \hat{r}, \quad \hat{r} \text { and } m \text { strongly commute. }
$$

Remark 6.19. The multiplicative unitary $\mathbb{W}$ is called type $I$ or type $I I$ when $\hat{r}=0$ or $\operatorname{ker}(\hat{r})=\{0\}$, respectively. Type I multiplicative unitaries are regular and modular while type II ones are manageable and nonregular. Finally, every type II multiplicative unitary can be obtained from one of type I (for details see [54]).

\subsubsection{Preparations on the algebraic level}

Let $0<q<1$ be the deformation parameter. The algebra of functions on the complex quantum plane is defined by

$$
K:=\mathbb{C}\left[\Upsilon, \Upsilon^{*}\right] /\left\langle\Upsilon^{*} \Upsilon=q^{2} \Upsilon \Upsilon^{*}\right\rangle
$$

The group algebra $\hat{A}=\mathbb{C} \mathbb{Z}$ is the universal algebra generated by a one-parameter group of elements $\left\{U_{n}\right\}_{n \in \mathbb{Z}}$. The Hopf *-algebra structure on $A$ is defined by

$$
\hat{\Delta}_{A}\left(U_{n}\right):=U_{n} \otimes U_{n}, \quad R_{\hat{A}}\left(U_{n}\right):=U_{n}^{*}, \quad e_{\hat{A}}\left(U_{n}\right):=1, \quad \text { for all } n \in \mathbb{Z} .
$$

There are a canonical right action $\gamma$ and a right coaction $\delta$ of $\hat{A}$ on $K$ defined by

$$
\gamma\left(\Upsilon, U_{n}\right):=q^{-2 n} \Upsilon \quad \text { and } \quad \delta(\Upsilon):=\Upsilon \otimes U_{1}^{*},
$$

for all $n \in \mathbb{Z}$.

Since $K$ is a $\mathbb{Z}$-graded $\mathbb{Z}$-module, it is a Yetter-Drinfeld module over $\left(\hat{A}, \hat{\Delta}_{A}\right)$. This allows to define the braided tensor product $K \otimes_{\text {alg }} K$. With the following structural maps, $K$ becomes a Hopf *-algebra in the monoidal category of $\left(\hat{A}, \hat{\Delta}_{A}\right)$-Yetter-Drinfeld modules:

$$
\Delta_{K}(\Upsilon):=\Upsilon \bigotimes_{\text {alg }} 1+1 \bigotimes_{\text {alg }} \Upsilon, \quad R_{K}\left(\Upsilon^{i}\right):=-\Upsilon^{i}, \quad e_{K}(\Upsilon):=0, \quad \text { for all } i \in \mathbb{Z} .
$$

Thus $\left(K, \Delta_{K}\right)$ is a braided Hopf ${ }^{*}$-algebra over $\left(\hat{A}, \hat{\Delta}_{A}\right)$.

Warning 6.20. In 20 the term braided bialgebra is used to denote quasitriangular bialgebras. This is because their categories of modules are braided. 


\subsubsection{Preparations on the Hilbert space level}

The quantum exponential functions $\mathrm{F}_{q}$ will play a central role in the construction of a braided multiplicative unitary for $\left(K, \Delta_{K}\right)$. Given a Hilbert space $\mathcal{H}$, define the operator domain

$$
\mathcal{E}_{\mathcal{H}}^{2}:=\left\{(R, S) \in \mathcal{E}_{\mathcal{H}} \times \mathcal{E}_{\mathcal{H}}: \begin{array}{l}
S R=q^{2} R S \\
S R^{*}=R^{*} S
\end{array}\right\} .
$$

The commutation relation in 6.68 explicitly means:

$$
\begin{array}{rr}
\text { Phase }(R) \text { Phase }(S)=\text { Phase }(S) \text { Phase }(R), & (\operatorname{Phase}(R))^{*}|S|(\operatorname{Phase}(R))=q|S|, \\
(\operatorname{Phase}(S))|R|(\operatorname{Phase}(S))^{*}=q|R|, & |R| \text { and }|S| \text { strongly commute. }
\end{array}
$$

Let us recall the following results on quantum exponential functions due to Woronowicz:

Theorem 6.21 (53, Theorem 2.1, 3.1]). Assume $(R, S) \in \mathcal{E}_{\mathcal{H}}^{2}$ and $\operatorname{ker}(R)=\operatorname{ker}(S)=\{0\}$. Then

$$
R \dot{+} S=\mathrm{F}_{q}\left(R^{-1} S\right) R \mathrm{~F}_{q}\left(R^{-1} S\right)^{*},
$$

and

$$
\mathrm{F}_{q}(R+S)=\mathrm{F}_{q}(R) \mathrm{F}_{q}(S) .
$$

where $\dot{+}$ denotes the closure of the sum.

The operator domain $\mathcal{F}_{\mathcal{H}}$ is defined by 6.62 . Here we define the following operator domains related to $\mathcal{F}_{\mathcal{H}}$ :

$$
\begin{aligned}
\mathcal{F}_{\mathcal{H}}^{*} & :=\left\{r \in \mathcal{C}(\mathcal{H}): \begin{array}{c}
r^{*} r=q^{2} r r^{*} \\
\operatorname{Sp}|r| \subset q^{\mathbb{Z}} \cup\{0\}
\end{array}\right\}, \\
\mathcal{F}_{\mathcal{H}}^{2} & :=\left\{(r, s) \in \mathcal{F}_{\mathcal{H}}^{*} \times \mathcal{F}_{\mathcal{H}}^{*}: \begin{array}{c}
q^{2} r s=s r \\
r^{*} s=q^{2} s r^{*}
\end{array}\right\} .
\end{aligned}
$$

Remark 6.22. The second domain $\sqrt{6.72}$ is not the same as in 54 . We have changed the second defining condition $s r^{*}=q^{2} r^{*} s$ to $q^{2} s r^{*}=r^{*} s$.

Lemma 6.23. $\mathcal{F}_{\mathcal{L}} \otimes \mathcal{F}_{\mathcal{H}}^{2} \subset \mathcal{E}_{\mathcal{L} \otimes \mathcal{H}}^{2}$.

Proof. Let $l \in \mathcal{F}_{\mathcal{L}}$ and $(r, s) \in \mathcal{F}_{\mathcal{H}}^{2}$ and define $(R, S):=(l \otimes r, l \otimes s)$. The following computation shows $R, S \in \mathcal{E}_{\mathcal{L} \otimes \mathcal{H}}$ :

$$
R^{*} R=\left(l^{*} l \otimes r^{*} r\right)=R R^{*} \quad \text { and } S^{*} S=\left(l^{*} l \otimes s^{*} s\right)=S S^{*} .
$$

Moreover,

$$
R^{*} S=\left(l^{*} l \otimes r^{*} s\right)=\left(l l^{*} \otimes s r^{*}\right)=S R^{*} \quad \text { and } \quad S R=\left(l^{2} \otimes s r\right)=q^{2}\left(l^{2} \otimes r s\right)=q^{2} R S .
$$

Let $\mathcal{L}$ be a Hilbert space and let $\Upsilon \in \mathcal{C}(\mathcal{H})$ be such that:

$$
\operatorname{ker}(\Upsilon)=\{0\}, \quad \operatorname{Sp}(|\Upsilon|) \subset \overline{\mathbb{C}}_{(q)}, \quad(\operatorname{Phase}(\Upsilon))|\Upsilon|(\operatorname{Phase}(\Upsilon))^{*}=q^{-1}|\Upsilon|
$$

The second condition is called spectral condition. It cannot be guessed from Hopf*-algebraic relations.

Let $\mathcal{H}=l^{2}(\mathbb{Z})$ and let $\left\{e_{i}\right\}_{i \in \mathbb{Z}}$ be an orthonormal basis of $\mathcal{H}$. Define

$$
u e_{i}:=e_{i+1} \quad \text { and } \quad \hat{N} e_{i}:=i e_{i} .
$$

Hence $u$ is unitary, $\hat{N}$ is a self-adjoint operator with $\operatorname{Sp}(\hat{N}) \subset \mathbb{Z}$, and

$$
u^{*} \hat{N} u=\hat{N}+1 \text {. }
$$

A computation in Section 6.2 .2 shows that $\mathbb{W}^{A}:=(I \otimes u)^{\hat{N} \otimes I}$ is a multiplicative unitary for the quantum group $\mathbb{G}=\left(\mathrm{C}(\mathbb{T}), \Delta_{\mathrm{C}(\mathbb{T})}\right)$, where $\Delta_{\mathrm{C}(\mathbb{T})}(u):=u \otimes u$. Since $\hat{N}$ has integer spectrum it is affiliated to $\mathrm{C}_{0}(\mathbb{Z})$. 
Thus $\widehat{\mathbb{G}}=\left(\mathrm{C}_{0}(\mathbb{Z}), \Delta_{\mathrm{C}_{0}(\mathbb{Z})}\right)$, where $\Delta_{\mathrm{C}_{0}(\mathbb{Z})}(\hat{N}):=\hat{N} \otimes 1 \dot{+} 1 \otimes \hat{N}$ is affiliated to $\mathrm{C}_{0}(\mathbb{Z} \times \mathbb{Z})$; here $\dot{+}$ denotes the closure of the sum. Since $\mathbb{G}$ and $\widehat{\mathbb{G}}$ are commutative quantum groups, by 4.9 , any $\mathbb{G}$-Drinfeld pairs $(\rho, \hat{\rho})$ must commute. Let $(\rho, \hat{\rho})$ be a $\mathbb{G}$-Drinfeld pair acting on $\mathcal{L}$. Let $\mathscr{U}=\rho(u)$ and $\hat{\mathscr{N}}=\hat{\rho}(\hat{N})$. Then

$$
\mathscr{U} \hat{\mathscr{N}} \mathscr{U}^{*}=\hat{\mathscr{N}} \text {. }
$$

The natural actions of $\mathbb{Z}$ and $\mathbb{T}$ on the Hopf ${ }^{*}$-algebra $K$ lead to the following commutation relations:

$$
\begin{aligned}
\mathscr{U}|\Upsilon| \mathscr{U}^{*} & =q^{2}|\Upsilon|, & & \mathscr{U} \operatorname{Phase}(\Upsilon) \mathscr{U}^{*}=\operatorname{Phase}(\Upsilon), \\
\operatorname{Phase}(\Upsilon) \hat{\mathscr{N}} \operatorname{Phase}(\Upsilon)^{*} & =(\hat{\mathscr{N}}+1), & & \text { and }|\Upsilon| \text { and } \hat{\mathscr{N}} \text { strongly commute. }
\end{aligned}
$$

Proposition 6.24. There are two irreducible Hilbert spaces $\mathcal{L}_{p}\left(p=\right.$ even and odd), such that $\mathcal{L}_{p}$ has an orthonormal basis $\left\{e_{i, j}\right\}_{i, j \in \mathbb{Z} ; i+j}$ is $p$, finite linear combinations of $e_{i, j}$ form a core for $\hat{\mathscr{N}}, \Upsilon$ and

$$
\Upsilon e_{i, j}:=q^{j} e_{i-1, j+1}, \quad \mathscr{U} e_{i, j}:=e_{i, j-2}, \quad \hat{\mathscr{N}} e_{i, j}:=i e_{i, j} .
$$

Proof. The operators $\hat{\mathscr{N}}$ and $|\Upsilon|$ have discrete spectrum and strongly commute, hence they have a common eigenvector. An element $e \in \mathcal{L}$ is said to be a common eigenvector of the pair $(\hat{\mathscr{N}},|\Upsilon|)$ with eigenvalue $\left(j, q^{i}\right)$ if

$$
\hat{\mathscr{N}} e=j e \quad|\Upsilon| e=q^{i} e .
$$

By (6.73) and (6.77) Phase $(\Upsilon)$ multiplies eigenvalues of $|\Upsilon|$ by $q$ and decreases eigenvalues of $\hat{\mathscr{N}}$ by 1 . Similarly, by 6.76), $\mathscr{U}$ multiplies eigenvalues of $|\Upsilon|$ by $q^{-2}$ and leaves eigenvalues of $\hat{\mathscr{N}}$ unchanged.

Starting with a normalised common eigenvector of $(\hat{\mathscr{N}},|\Upsilon|)$ and applying an appropriate power of $\operatorname{Phase}(\Upsilon)$ we obtain an eigenvector of $(\hat{\mathscr{N}},|\Upsilon|)$ with eigenvalue $\left(0, q^{j}\right)$. Next applying an appropriate power of $\mathscr{U}$ we obtain a common eigenvector of $(\hat{\mathscr{N}},|\Upsilon|)$ with eigenvalue either $(0,1)$ or $(0, q)$ (depending on the parity of $j$ ).

Let $e_{0,0}$ be a common eigenvector of $(\hat{\mathscr{N}},|\Upsilon|)$ with eigenvalue $(0,1)$. Applying to $e_{00}$ powers of $\mathscr{U}$ and $\operatorname{Phase}(\Upsilon)$ gives a family of common eigenvectors $e_{i j}(i, j \in \mathbb{Z}, m+n$ is even) of $(\hat{N},|\Upsilon|)$ with eigenvalue $\left(i, q^{j}\right)$ :

$$
\mathscr{U} e_{i, j}=e_{i, j-2}, \quad(\operatorname{Phase}(\Upsilon)) e_{i, j}=e_{i-1, j+1} .
$$

If $\mathcal{L}$ is irreducible then $\left\{e_{i, j}\right\}$ ( $n, m \in \mathbb{Z}, m+n$ is even) is an orthonormal basis of $\mathcal{L}$.

Similarly, if $e_{0,1}$ is an eigenvector of $(\hat{\mathscr{N}},|\Upsilon|)$ with eigenvalue $(0, q)$ then applying to $e_{0,1}$ powers of $\mathscr{U}$ and Phase $(\Upsilon)$ gives a family of common eigenvectors $e_{i, j} n, m \in \mathbb{Z}, m+n$ is odd of $(\hat{\mathscr{N}},|\Upsilon|)$ with eigenvalue $\left(i, q^{j}\right)$. Again, if $\mathcal{L}$ is irreducible then $\left\{e_{i, j}\right\}(n, m \in \mathbb{Z}, m+n$ is odd) is an orthonormal basis of $\mathcal{L}$.

Proposition 6.25. Let $\Upsilon, \mathscr{U}$ and $\hat{\mathscr{N}}$ act on $\mathcal{L}$ with the above commutation relations 6.75 -6.77.

$$
\mathrm{F}_{q}\left(\Upsilon^{-1} q^{-2 \hat{\mathscr{N}}} \otimes \Upsilon\right)(\Upsilon \otimes 1) \mathrm{F}_{q}\left(\Upsilon^{-1} q^{-2 \hat{\mathscr{N}}} \otimes \Upsilon\right)^{*}=\Upsilon \otimes 1 \dot{+} q^{-2 \hat{\mathscr{N}}} \otimes \Upsilon
$$

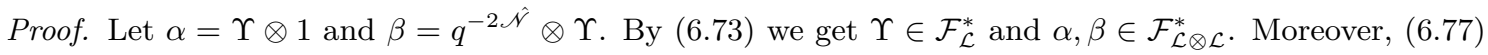
implies

and

$$
\beta \alpha=\left(q^{-2 \hat{\mathscr{N}}} \Upsilon \otimes \Upsilon\right)=\left(\Upsilon q^{-2(\hat{\mathscr{N}}-1)} \otimes \Upsilon\right)=q^{2} \alpha \beta
$$

hence $(\alpha, \beta) \in \mathcal{F}_{\mathcal{L}}^{2}$.

$$
\alpha^{*} \beta=\left(\Upsilon^{*} q^{-2 \hat{N}} \otimes \Upsilon\right)=q^{2} \beta \alpha^{*}
$$

Let $l \in \mathcal{F}_{\mathcal{B}}$ satisfy $\operatorname{ker}(l)=\{0\}$. Lemma 6.23 gives $(R, S) \in \mathcal{E}_{\mathcal{B} \otimes \mathcal{L}}^{2}$, where $R:=l \otimes \alpha$ and $S:=l \otimes \beta$. Then Theorem 6.21 yields:

$$
\mathrm{F}_{q}\left(1 \otimes \alpha^{-1} \beta\right)(l \otimes \alpha) \mathrm{F}_{q}\left(1 \otimes \alpha^{-1} \beta\right)^{*}=l \otimes \alpha \dot{+} l \otimes \beta .
$$

Since $l \in \mathcal{F}_{\mathcal{B}}$ is arbitrary, we get 6.79. 
Corollary 6.26. Let $\hat{r} \in \mathcal{C}_{\mathcal{L}}$ such that

Phase $(\Upsilon) \operatorname{Phase}(\hat{r}) \operatorname{Phase}(\Upsilon)^{*}=q^{2} \operatorname{Phase}(\hat{r}), \quad$ and $|\Upsilon|$ and $|\hat{r}|$ strongly commute.

If $\Upsilon^{-1} q^{-2 \hat{\mathcal{N}}} \otimes \Upsilon \dot{+} \hat{r}$ exists, then

$$
\mathrm{F}_{q}\left(\Upsilon^{-1} q^{-2 \hat{\mathcal{N}}}+q^{-2 \hat{\mathcal{N}}} \hat{r} \otimes \Upsilon\right)(\Upsilon \otimes 1) \mathrm{F}_{q}\left(\Upsilon^{-1} q^{-2 \hat{\mathcal{N}}} \dot{+} q^{-2 \hat{\mathcal{N}}} \hat{r} \otimes \Upsilon\right)^{*}=\Upsilon \otimes 1+q^{-2 \hat{\mathcal{N}}} \otimes \Upsilon .
$$

Proof. The property 6.70$)$ of $\mathrm{F}_{q}$ implies

$$
\mathrm{F}_{q}\left(\Upsilon^{-1} q^{-2 \hat{\mathcal{N}}}+q^{-2 \hat{N}} \hat{r} \otimes \Upsilon\right) \mathrm{F}_{q}\left(\Upsilon^{-1} q^{-2 \hat{N}} \otimes \Upsilon\right)=\mathrm{F}_{q}\left(q^{-2 \hat{\mathscr{N}}} \hat{r} \otimes \Upsilon\right) .
$$

By Proposition 6.25 it suffices to show $\mathrm{F}_{q}\left(q^{-2 \hat{\mathcal{N}}} \hat{r} \otimes \Upsilon\right)(\Upsilon \otimes 1) \mathrm{F}_{q}\left(q^{-2 \hat{\mathscr{N}}} \hat{r} \otimes \Upsilon\right)^{*}=\Upsilon \otimes 1$.

The commutation relations 6.77) and 6.80) imply $\left(q^{-2 \hat{N}} \hat{r} \otimes \Upsilon\right)(\Upsilon \otimes 1)=\left(q^{-2 \hat{N}-2} \Upsilon \hat{r} \otimes \Upsilon\right)=$ $(\Upsilon \otimes 1)\left(q^{-2 \hat{N}} \hat{r} \otimes \Upsilon\right)$.

\subsubsection{Braided quantum group structure on the quantum plane}

In this section we shall describe the $\mathrm{C}^{*}$-algebraic version of $\left(K, \Delta_{K}\right)$ as a braided quantum group over $\mathbb{G}$.

Consider the polar decomposition of $\Upsilon^{*}=v h$, where $v=\operatorname{Phase}\left(\Upsilon^{*}\right)$ is unitary and $h=\left|\Upsilon^{*}\right|$ is a positive self-adjoint operator on a Hilbert space $\mathcal{L}$. Then $\operatorname{Sp}(h) \subset q^{\mathbb{Z}} \cup\{0\}$ and $\operatorname{Ker}(h)=\{0\}$. Define

$$
K_{0}:=\left\{\sum_{k \in \mathbb{Z}}^{\text {finite }} v^{k} f_{k}(h): \begin{array}{c}
f_{k} \in \mathrm{C}_{0}\left(\overline{\mathbb{C}}_{(q)}\right) \\
f_{k}(0)=0 \text { for } k \neq 0
\end{array}\right\} .
$$

Proposition 4.1 in 37 implies:

Proposition 6.27. Let $K$ be the norm closure of $K_{0}$. Then

1. $K$ is a $\mathrm{C}^{*}$-algebra.

2. $\Upsilon^{*}$ is affiliated to $K$.

3. $K$ is generated by $\Upsilon^{*}$.

Define the following coactions $\gamma(\Upsilon):=\Upsilon \otimes u^{*}$ affiliated to $K \otimes C(\mathbb{T})$ and $\hat{\gamma}(\Upsilon):=\Upsilon \otimes q^{-2 \hat{N}}$ affiliated to $K \otimes \mathrm{C}_{0}(\mathbb{Z})$.

Define $\mathrm{U}:=(1 \otimes u)^{\hat{\mathcal{r}} \otimes 1} \in \mathcal{U}(\mathbb{K}(\mathcal{L}) \otimes \mathrm{C}(\mathbb{T}))$ and $\hat{\mathrm{V}}:=(1 \otimes \mathscr{U})^{\hat{N} \otimes 1} \in \mathcal{U}\left(\mathrm{C}_{0}(\mathbb{Z}) \otimes \mathbb{K}(\mathcal{L})\right)$.

Since $\mathscr{U}$ and $\hat{\mathscr{N}}$ commute, $(\mathrm{U}, \mathrm{V})$ is a $\mathbb{G}$-Yetter-Drinfeld pair, where $\mathrm{V}=\left(\mathscr{U}^{*} \otimes 1\right)^{1 \otimes \hat{N}} \in \mathcal{U}\left(\mathbb{K}(\mathcal{L}) \otimes \mathrm{C}_{0}(\mathbb{Z})\right)$. Then $Z:=(\hat{\rho} \otimes \rho)\left(\left(1 \otimes u^{*}\right)^{\hat{N} \otimes 1}\right)=\left(1 \otimes \mathscr{U}^{*}\right)^{\hat{\mathcal{N}} \otimes 1}$. The corresponding braiding operator ${ }^{\mathcal{L}} \bigvee^{\mathcal{L}} \in \mathcal{U}(\mathcal{L} \otimes \mathcal{L})$ is defined by

$$
\mathcal{L}^{\mathcal{L}} \boldsymbol{X}^{\mathcal{L}}:=\left(1 \otimes \mathscr{U}^{*}\right)^{\mathcal{H} \otimes 1} \circ \Sigma
$$

Lemma 6.28. Let $\varphi$ denote the representation $K \rightarrow \mathbb{B}(\mathcal{L})$. Then $(\varphi, \mathrm{U})$ and $(\varphi, \mathrm{V})$ are covariant representations of $(K, \gamma, \mathrm{C}(\mathbb{T}))$ and $\left(K, \hat{\gamma}, \mathrm{C}_{0}(\mathbb{Z})\right)$.

Proof. The commutation relation (6.76) between $\mathscr{U}$ and $\Upsilon$ yields:

$$
\left(I \otimes \mathscr{U}^{*}\right)^{\hat{N} \otimes I}(I \otimes \Upsilon)(I \otimes \mathscr{U})^{\hat{N} \otimes I}=q^{-2 \hat{N}} \otimes \Upsilon .
$$

Hence $\mathrm{V}(\Upsilon \otimes 1) \mathrm{V}^{*}=\Upsilon \otimes q^{-2 \hat{N}}=\hat{\gamma}(\Upsilon)$.

Similarly, the commutation relation 6.77 ) gives

$$
(I \otimes u)^{\hat{\mathcal{N}} \otimes I}(\Upsilon \otimes I)\left(I \otimes u^{*}\right)^{\hat{\mathcal{N}} \otimes I}=\Upsilon \otimes u^{*},
$$

which is equivalent to $\mathrm{U}(\Upsilon \otimes 1) \mathrm{U}=\Upsilon \otimes u^{*}=\gamma(\Upsilon)$. 
The crossed product $K \otimes K$ is $\iota_{1}(K) \cdot \iota_{2}(K)$, where $\iota_{1}(\Upsilon):=\Upsilon \otimes 1$ and $\iota_{2}(\Upsilon):=\stackrel{\mathcal{L}}{\Upsilon}^{\mathcal{L}}(\Upsilon \otimes 1)\left(\mathrm{X}^{\mathcal{L}}\right)^{*}$.

Theorem 6.29. In the notation introduced above, we have:

1. the sum $\iota_{1}(\Upsilon) \dot{+} \iota_{2}(\Upsilon)$ is affiliated to $K \otimes K$ and $\operatorname{Sp}\left(\iota_{1}(\Upsilon) \dot{+} \iota_{2}(\Upsilon)\right) \subset \overline{\mathbb{C}}_{(q)}$.

2. there is a unique $\mathbb{Z} \times \mathbb{T}$-equivariant morphism $\Delta_{K} \in \operatorname{Mor}(K, K \otimes K)$ such that

$$
\Delta_{K}(\Upsilon)=\iota_{1}(\Upsilon) \dot{+} \iota_{2}(\Upsilon)
$$

3. $\Delta_{K}$ is coassociative.

Proof. The defining condition $(6.82)$ of ${ }^{\mathcal{L}} \Upsilon^{\mathcal{L}}$ suggests $Z=\left(\operatorname{id}_{\mathcal{L}} \otimes \hat{\rho}\right)$ V. Therefore, $(6.83)$ gives $\iota_{2}(\Upsilon):=$ $q^{-2 \hat{N}} \otimes \Upsilon$. Now $\Upsilon \otimes 1$ is affiliated to $K \otimes K$, and by Proposition $6.25 \Upsilon \otimes 1$ is unitarily equivalent to $\iota_{1}(\Upsilon) \dot{+} \iota_{2}(\Upsilon)$; hence $\iota_{1}(\Upsilon) \dot{+} \iota_{2}(\Upsilon)$ it is affiliated to $K \otimes K$.

Since $\mathbb{G}$ and $\widehat{\mathbb{G}}$ are abelian, Yetter-Drinfeld $\mathrm{C}^{*}$-algebras over $\mathbb{G}$ are the same as $\mathrm{C}_{0}(\mathbb{Z} \times \mathbb{T})$-C $\mathrm{C}^{*}$-algebras.

Equation 6.79 shows that $\Delta_{K}$ is implemented by $\mathrm{F}_{q}\left(\Upsilon^{-1} q^{-2 \hat{N}} \otimes \Upsilon\right)$.

Finally, the coassociativity of $\Delta_{K}$ means that $\left(\iota_{1}(\Upsilon) \dot{+} \iota_{2}(\Upsilon)\right) \dot{+} \iota_{3}(\Upsilon)=\iota_{1}(\Upsilon) \dot{+}\left(\iota_{2}(\Upsilon) \dot{+} \iota_{3}(\Upsilon)\right)$, where the $\iota_{j}$ s are the canonical embeddings of $K$ into $K \otimes K \otimes K$ for $j=1,2,3$. This follows from (6.70) and Proposition 6.25 .

Since $(\mathrm{U}, \mathrm{V})$ is a $\mathbb{G}$-Yetter-Drinfeld pair, Lemma 6.28 gives $K \in \mathcal{Y} \mathcal{D} \mathfrak{C}^{*} \mathfrak{a l g}(\mathbb{G})$. In order to show that $\left(K, \Delta_{K}\right)$ is a braided quantum group in the sense of Definition 6.5 we need to show that $\mathrm{F}_{q}\left(\Upsilon^{-1} q^{-2 \hat{\mathcal{N}}} \otimes\right.$ $\Upsilon)$ is a braided multiplicative unitary over $\mathbb{G}$.

Proposition 6.30. The unitary $\mathbb{F}:=\mathrm{F}_{q}\left(\Upsilon^{-1} q^{-2 \hat{N}} \otimes \Upsilon\right) \in \mathcal{U}(\mathcal{L} \otimes \mathcal{L})$ is a braided multiplicative unitary over $\mathbb{G}$.

Proof. Theorem 6.29, 2 ) and the property 6.70 of $\mathrm{F}_{q}$ imply

$$
\begin{aligned}
&\left(\mathrm{id} \otimes \Delta_{K}\right) \mathbb{F}=\mathrm{F}_{q}\left(\Upsilon^{-1} q^{-2 \hat{\mathcal{N}}} \otimes\left(\iota_{1}(\Upsilon)+\iota_{2}(\Upsilon)\right)=\mathrm{F}_{q}\left(\Upsilon^{-1} q^{-2 \hat{\mathcal{N}}} \otimes \iota_{1}(\Upsilon)\right) \mathrm{F}_{q}\left(\Upsilon^{-1} q^{-2 \hat{\mathcal{N}}} \otimes \iota_{2}(\Upsilon)\right)\right. \\
&=\mathbb{F}_{12} Z_{23} \mathbb{F}_{13} Z_{23}^{*} .
\end{aligned}
$$

Since $\Delta_{K}$ is implemented by $\mathrm{F}_{q}\left(\Upsilon^{-1} q^{-2 \hat{N}} \otimes \Upsilon\right)$ we also have

$$
\left(\mathrm{id} \otimes \Delta_{K}\right) \mathbb{F}=\mathbb{F}_{23} \mathbb{F}_{12} \mathbb{F}_{23}^{*} \text {. }
$$

Comparing the right hand sides of the last two equations gives the braided pentagon equation 6.6 for $\mathbb{F}$.

The commutation relation 6.77 between $\Upsilon$ and $\hat{\mathscr{N}}$ gives

$$
\begin{aligned}
& \left(\Upsilon^{-1} q^{-2 \hat{\mathscr{N}}} \otimes \Upsilon \otimes 1\right)(1 \otimes 1 \otimes u)^{\hat{\mathscr{N}} \otimes 1 \otimes 1}(1 \otimes 1 \otimes u)^{1 \otimes \hat{\mathscr{N}} \otimes 1} \\
& =(1 \otimes 1 \otimes u)^{(\hat{N}-1) \otimes 1 \otimes 1}\left(\Upsilon^{-1} q^{-2 \hat{\mathscr{N}}} \otimes \Upsilon \otimes 1\right)(1 \otimes 1 \otimes u)^{1 \otimes \hat{\mathscr{N}} \otimes 1} \\
& =(1 \otimes 1 \otimes u)^{\hat{\mathscr{N}} \otimes 1 \otimes 1}(1 \otimes 1 \otimes u)^{1 \otimes \hat{N} \otimes 1}\left(\Upsilon^{-1} q^{-2 \hat{N}} \otimes \Upsilon \otimes 1\right) .
\end{aligned}
$$

Therefore, we obtain 6.3. Similar routine computations yield 6.4.

\subsubsection{Construction of simplified quantum $\mathrm{E}(2)$}

Now we shall construct simplified $\mathrm{E}_{q}(2)$ from $\left(K, \Delta_{K}\right)$ and $\mathbb{G}$. Moreover, we show that the multiplicative unitary defined by 6.55, constructed out of the braided multiplicative unitary $\mathbb{F}$ in Proposition 6.30 and $(1 \otimes u)^{\hat{N} \otimes 1}$, generates simplified $\mathrm{E}_{q}(2)$.

Define an action of $\mathbb{Z}$ on $K$ by $\psi_{n}(\Upsilon)=q^{-2 n} \Upsilon$. Let $C=K \rtimes \mathbb{Z}$ be the corresponding crossed product. The *-homomorphism $K \hookrightarrow C$ is nondegenerate, and there is a unitary $w \in \mathcal{M}(C)$ such that $w|\Upsilon| w^{*}=q^{2}|\Upsilon|$ and $w \operatorname{Phase}(\Upsilon) w^{*}=\operatorname{Phase}(\Upsilon)$. Thus $C$ is the universal object generated by $w$ and $\Upsilon$. Realise $w$ and $\Upsilon$ as a unitary and an unbounded operator on a Hilbert space $\mathcal{H}$. Then $(6.73)$ gives $(w, \Upsilon) \in \mathcal{G}_{\mathcal{H}}$; hence $C$ is the underlying $\mathrm{C}^{*}$-algebra of simplified $\mathrm{E}_{q}(2)$.

Realise $\mathrm{U}$ and $\hat{\mathrm{V}}$ as operators defined by $\mathbb{U}:=(1 \otimes u)^{\hat{\mathcal{N}} \otimes 1} \in \mathcal{U}(\mathcal{L} \otimes \mathcal{H})$ and $\hat{\mathbb{V}}:=\left(1 \otimes \mathscr{U}^{*}\right)^{\hat{N} \otimes 1} \in \mathcal{U}(\mathcal{H} \otimes \mathcal{L})$. 
Proposition 6.31. The unitary $\mathbb{W}_{1234}:=\mathbb{W}_{13}^{A} \mathbb{U}_{23} \hat{\mathbb{V}}_{34}^{*} \mathbb{F}_{24} \hat{\mathbb{V}}_{34} \in \mathcal{U}(\mathcal{H} \otimes \mathcal{L} \otimes \mathcal{H} \otimes \mathcal{L})$ is a type-I multiplicative unitary generating simplified $\mathrm{E}_{q}(2)$.

Proof. Equation 6.83 implies

$$
\left(1 \otimes \mathscr{U}^{*}\right)^{\hat{N} \otimes 1}(1 \otimes \Upsilon)(1 \otimes \mathscr{U})^{\hat{N} \otimes 1}=q^{-2 \hat{N}} \otimes \Upsilon .
$$

Therefore, $\hat{\mathbb{V}}_{34}^{*} \mathbb{F}_{24} \hat{\mathbb{V}}_{34}=\mathrm{F}_{q}\left(1 \otimes \Upsilon^{-1} q^{-2 \hat{N}} \otimes q^{-2 \hat{N}} \otimes \Upsilon\right)$

Next 6.77) implies

$$
\begin{aligned}
(1 \otimes u)^{\hat{\mathcal{N}} \otimes 1}\left(\Upsilon^{-1} q^{-2 \hat{\mathcal{N}}} \otimes q^{-2 \hat{N}}\right) & =(1 \otimes u)^{\hat{\mathcal{N}} \otimes 1}\left(\Upsilon^{-1} q^{-2 \hat{\mathcal{N}}} \otimes 1\right)\left(1 \otimes q^{-2 \hat{N}}\right) \\
& =\left(\Upsilon^{-1} q^{-2 \hat{\mathcal{N}}} \otimes 1\right)(1 \otimes u)^{(\hat{\mathcal{N}}+1) \otimes 1}\left(1 \otimes q^{-2 \hat{N}}\right) \\
& =\left(\Upsilon^{-1} q^{-2 \hat{\mathcal{N}}} \otimes 1\right)\left(1 \otimes q^{-2 \hat{N}}\right)\left(q^{2} \otimes u\right)^{(\hat{N}+1) \otimes 1} \\
& =\left(\Upsilon^{-1} q^{-2 \hat{N}} \otimes 1\right)\left(1 \otimes q^{-2(\hat{N}-1)} u\right)\left(q^{2} \otimes u\right)^{\hat{N} \otimes 1} \\
& =\left(\Upsilon^{-1} q^{-2 \hat{N}} \otimes u q^{-2 \hat{N}}\right)\left(q^{2} \otimes u\right)^{\hat{N} \otimes 1} \\
& =\left(\Upsilon^{-1} \otimes u q^{-2 \hat{N}}\right)(1 \otimes u)^{\hat{N} \otimes 1}
\end{aligned}
$$

Hence $\mathbb{U}_{23} \hat{\mathbb{V}}_{34}^{*} \mathbb{F}_{24} \hat{\mathbb{V}}_{34}=\mathrm{F}_{q}\left(1 \otimes \Upsilon^{-1} \otimes u q^{-2 \hat{N}} \otimes \Upsilon\right)(1 \otimes 1 \otimes u \otimes 1)^{1 \otimes \hat{\mathscr{N}} \otimes 1 \otimes 1}$.

Equation (6.74) implies

$$
(1 \otimes u)^{\hat{N} \otimes 1}\left(1 \otimes u q^{-2 \hat{N}}\right)=\left(1 \otimes u q^{-2 \hat{N}}\right)\left(q^{2} \otimes u\right)^{\hat{N} \otimes 1}=\left(q^{2 \hat{N}} \otimes u q^{-2 \hat{N}}\right)(1 \otimes u)^{\hat{N} \otimes 1} .
$$

Therefore, $\mathbb{W}_{1234}=\mathrm{F}_{q}\left(q^{2 \hat{N}} \otimes \Upsilon^{-1} \otimes u q^{-2 \hat{N}} \otimes \Upsilon\right)(1 \otimes 1 \otimes u \otimes 1)^{(\hat{N} \otimes 1 \otimes 1 \otimes 1}(1 \otimes 1 \otimes u \otimes 1)^{1 \otimes \hat{N} \otimes 1 \otimes 1}$.

Since $(1 \otimes u)^{\hat{N} \otimes 1}$ is a multiplicative unitary for $\mathbb{G}, \hat{\mathrm{V}}^{*}(1 \otimes \hat{\mathscr{N}}) \hat{\mathrm{V}}=\hat{N} \otimes 1 \dot{+} 1 \otimes \hat{\mathscr{N}}$, and $\operatorname{Sp}(\hat{N} \otimes 1 \dot{+} 1 \otimes \hat{\mathscr{N}}) \subset$

Thus $\mathbb{W}_{1234}=\mathrm{F}_{q}\left(q^{2 \hat{N}} \otimes \Upsilon^{-1} \otimes u q^{-2 \hat{N}} \otimes \Upsilon\right)(1 \otimes 1 \otimes u \otimes 1)^{(\hat{N} \otimes 1+1 \otimes \hat{N}) \otimes 1 \otimes 1}$

Define $m^{\prime}:=u q^{-2 \hat{N}} \otimes \Upsilon, u^{\prime}:=u \otimes 1$, and $\hat{N}^{\prime}:=\hat{N} \otimes 1+1 \otimes \hat{\mathscr{N}}$. Then $\operatorname{ker}\left(m^{\prime}\right)=\{0\}, \operatorname{Sp}\left(m^{\prime}\right) \subset \overline{\mathbb{C}}_{(q)}$, and 6.76) gives $u^{\prime} m^{\prime} u^{\prime *}=u u q^{-2 \hat{N}} u^{*} \otimes \Upsilon=u q^{-2 \hat{N}+2} \otimes \Upsilon=q^{2} m^{\prime}$; hence $\left(u^{\prime}, m^{\prime}\right) \in \mathcal{G}_{\mathcal{H} \otimes \mathcal{L}}$. Similarly, 6.74) gives $u^{\prime *} N^{\prime} u^{\prime}=\hat{N}^{\prime}+1$. Finally, 6.74 and 6.77) show that the operators $m^{\prime}$ and $\hat{N}^{\prime}$ strongly commute.

Therefore, by Theorem $6.18 \mathbb{W}_{1234}=\mathrm{F}_{q}\left(m^{\prime-1} u^{\prime} \otimes m^{\prime}\right)$ is a multiplicative unitary of type I.

By Remark 6.19 evey type II multiplicative unitary can be constructed from one of type I. Alternatively, if we start from a multiplicative unitary as in Corollary 6.26 a similar computation shows that $\mathbb{W}_{1234}$ is of type II. Further, using 6.77) and 6.76 we get $Z^{*} \mathbb{F}=\mathrm{F}_{q}\left(\Upsilon^{-1} \dot{+} \hat{r} \otimes \mathscr{U} \Upsilon\right)(1 \otimes \mathscr{U})^{\hat{N} \otimes 1}$. Therefore, $\mathbb{F}$ is manageable if $\mathbb{W}_{1234}$ is. 


\section{Bibliography}

[1] Saad Baaj, Représentation régulière du groupe quantique $E_{\mu}(2)$ de Woronowicz, C. R. Acad. Sci. Paris Sér. I Math. 314 (1992), no. 13, 1021-1026. MR 1168528

[2] Saad Baaj and Georges Skandalis, Unitaires multiplicatifs et dualité pour les produits croisés de $C^{*}$-alqèbres, Ann. Sci. École Norm. Sup. (4) 26 (1993), no. 4, 425-488, available at http://www.numdam.org/item?id=ASENS_ 1993_4_26_4_425_0 MR 1235438

[3] Saad Baaj, Georges Skandalis, and Stefaan Vaes, Non-semi-regular quantum groups coming from number theory, Comm. Math. Phys. 235 (2003), no. 1, 139-167, doi: 10.1007/s00220-002-0780-6 MR 1969723

[4] Saad Baaj and Stefaan Vaes, Double crossed products of locally compact quantum groups, J. Inst. Math. Jussieu 4 (2005), no. 1, 135-173, doi: 10.1017/S1474748005000034 MR 2115071

[5] Vyjayanthi Chari and Andrew Pressley, A guide to quantum groups, Cambridge University Press, Cambridge, 1994. MR 1300632

[6] Alain Connes, Noncommutative geometry, Academic Press Inc., San Diego, CA, 1994. MR 1303779

[7] A. Van Daele, Multiplier Hopf algebras, Trans. Amer. Math. Soc. 342 (1994), no. 2, 917-932, doi: 10.2307/2154659 MR 1220906

[8] , An algebraic framework for group duality, Adv. Math. 140 (1998), no. 2, 323-366, doi: 10.1006/aima.1998.1775 1658585 (2000g:16045)

[9] Matthew Daws, Paweł Kasprzak, Adam Skalski, and Piotr M. Sołtan, Closed quantum subgroups of locally compact quantum groups, Adv. Math. 231 (2012), no. 6, 3473-3501, doi: 10.1016/j.aim.2012.09.002 MR 2980506

[10] Jacques Dixmier, $C^{*}$-Algebras, North-Holland Publishing Co., Amsterdam, 1977. Translated from the French by Francis Jellett; North-Holland Mathematical Library, Vol. 15. MR 0458185

[11] V. G. Drinfeld, Quantum groups, Proceedings of the International Congress of Mathematicians, Vol. 1, 2 (Berkeley, Calif., 1986), 1987, pp. 798-820. MR 934283

[12] _ Almost cocommutative Hopf algebras, Algebra i Analiz 1 (1989), no. 2, 30-46. MR 1025154

[13] Ruy Exel, Blends and alloys, C. R. Math. Acad. Sci. Soc. R. Can. 35 (2013), no. 3, 77-113. MR 3136103

[14] Gerald B. Folland, A course in abstract harmonic analysis, Studies in Advanced Mathematics, CRC Press, Boca Raton, FL, 1995. MR 1397028

[15] P. Ghez, Ricardo Lima, and John E. Roberts, $W^{*}$-categories, Pacific J. Math. 120 (1985), no. 1, 79-109, available at http://projecteuclid.org/euclid.pjm/1102703884 MR 808930

[16] Michio Jimbo, A q-difference analogue of $U(\mathfrak{g})$ and the Yang-Baxter equation, Lett. Math. Phys. 10 (1985), no. 1, 63-69, doi: 10.1007/BF00704588 MR 797001

[17] André Joyal and Ross Street, Braided tensor categories, Adv. Math. 102 (1993), no. 1, 20-78, doi: 10.1006/aima.1993.1055 MR 1250465

[18] Gennadi G. Kasparov, The operator K-functor and extensions of $C^{*}$-algebras, Izv. Akad. Nauk SSSR Ser. Mat. 44 (1980), no. 3, 571-636, 719, available at http://mi.mathnet.ru/izv1739 English transl., Math. USSR-Izv. 16 (1981), no. 3, 513-572 (1981). MR 582160

[19] Paweł Kasprzak, Rieffel deformation of homogeneous spaces, J. Funct. Anal. 260 (2011), no. 1, 146-163, doi: 10.1016/j.jfa.2010.08.009 MR 2733574

[20] Christian Kassel, Quantum groups, Graduate Texts in Mathematics, vol. 155, Springer-Verlag, New York, 1995., doi: 10.1007/978-1-4612-0783-2 MR 1321145

[21] Johan Kustermans, KMS weights on $C^{*}$-algebras (1997), eprint. arXiv:math/9704008 
[22] _ Locally compact quantum groups in the universal setting, Internat. J. Math. 12 (2001), no. 3, 289-338, doi: 10.1142/S0129167X01000757 MR 1841517

[23] Johan Kustermans and Stefaan Vaes, Weight theory for $C^{*}$-algebraic quantum groups (1999), eprint. arXiv:math/9901063

[24] , Locally compact quantum groups, Ann. Sci. École Norm. Sup. (4) 33 (2000), no. 6, 837-934, doi: 10.1016/S0012-9593(00)01055-7 MR 1832993

[25] _ Locally compact quantum groups in the von Neumann algebraic setting, Math. Scand. 92 (2003), no. 1, 68-92. MR 1951446

[26] Shahn Majid, Foundations of quantum group theory, Cambridge University Press, Cambridge, 1995., doi: 10.1017/CBO9780511613104 MR 1381692

[27] T Masuda, Y Nakagami, and Stanisław Lech Woronowicz, A $C^{*}$-algebraic framework for quantum groups, Internat. J. Math 14 (2003), no. 9, 903-1001, doi: 10.1142/S0129167X03002071. MR 2020804

[28] Ralf Meyer, Sutanu Roy, and Stanisław Lech Woronowicz, Homomorphisms of quantum groups, Münster J. Math. 5 (2012), 1-24, available at http://wwwmath1.uni-muenster.de/mjm/vol_5/mjm_vol_5_01.pdf

[29] , Quantum group-twisted tensor products of $\mathrm{C}^{*}$-algebras, Internat. J. Math. 25 (2014), no. 2, 1450019, doi: $10.1142 / \mathrm{S} 0129167 \mathrm{X} 14500190$

[30] Ryszard Nest and Christian Voigt, Equivariant Poincaré duality for quantum group actions, J. Funct. Anal. 258 (2010), no. 5, 1466-1503, doi: 10.1016/j.jfa.2009.10.015 MR 2566309

[31] Chi-Keung Ng, Morphisms of multiplicative unitaries, J. Operator Theory 38 (1997), no. 2, 203-224, available at http://www .mathjournals.org/jot/1997-038-002/1997-038-002-001.pdf MR 1606928

[32] Katsumi Numakura, On bicompact semigroups, Math. J. Okayama Univ. 1 (1952), 99-108. MR 0048467

[33] Gert K. Pedersen, $C^{*}$-algebras and their automorphism groups, London Mathematical Society Monographs, vol. 14, Academic Press, London, 1979. MR 548006

[34] Piotr Podleś and Stanisław Lech Woronowicz, Quantum deformation of Lorentz group, Comm. Math. Phys. 130 (1990), no. 2, 381-431, available at http://projecteuclid.org/euclid.cmp/1104200517 MR 1059324

[35] David E. Radford, The structure of Hopf algebras with a projection, J. Algebra 92 (1985), no. 2, 322-347, doi: 10.1016/0021-8693(85)90124-3. MR 778452

[36] Piotr Stachura, Towards a topological (dual of) quantum $\kappa$-Poincaré group, Rep. Math. Phys. 57 (2006), no. 2, 233-256, doi: 10.1016/S0034-4877(06)80019-4, MR 2227008

[37] Piotr Mikołaj Sołtan, Examples of non-compact quantum group actions, J. Math. Anal. Appl. 372 (2010), no. 1, 224-236, doi: 10.1016/j.jmaa.2010.06.045 MR 2672521

[38] Piotr Mikołaj Sołtan and Stanisław Lech Woronowicz, A remark on manageable multiplicative unitaries, Lett. Math. Phys. 57 (2001), no. 3, 239-252, doi: 10.1023/A:1012230629865. MR 1862455

[39] . From multiplicative unitaries to quantum groups. II, J. Funct. Anal. 252 (2007), no. 1, 42-67, doi: 10.1016/j.jfa.2007.07.006 MR 2357350

[40] Thomas Timmermann, An invitation to quantum groups and duality, European Mathematical Society (EMS), Zürich, 2008., doi: 10.4171/043 MR 2397671

[41] Stefaan Vaes, A new approach to induction and imprimitivity results, J. Funct. Anal. 229 (2005), no. 2, 317-374, doi: 10.1016/j.jfa.2004.11.016 MR 2182592

[42] Stefaan Vaes and Leonid Vainerman, Extensions of locally compact quantum groups and the bicrossed product construction, Adv. Math. 175 (2003), no. 1, 1-101, doi: 10.1016/S0001-8708(02)00040-3 MR 1970242

[43] Shuzhou Wang, Free products of compact quantum groups, Comm. Math. Phys. 167 (1995), no. 3, 671-692, available at http://projecteuclid.org/getRecord?id=euclid.cmp/1104272163 MR 1316765

[44] Stanisław Lech Woronowicz, An example of a braided locally compact group, Quantum Groups: Formalism and Applications, XXX Karpacz Winter School (Karpacz, 1994), PWN, Warsaw, 1995, pp. 155-171. MR 1647968

[45] _ Compact matrix pseudogroups, Comm. Math. Phys. 111 (1987), no. 4, 613-665, available at http: //projecteuclid.org/euclid.cmp/1104159726. MR 901157

[46] _ $C^{*}$-algebras generated by unbounded elements, Rev. Math. Phys. 7 (1995), no. 3, 481-521, doi: 10.1142/S0129055X95000207. MR 1326143

[47] Unbounded elements affiliated with $C^{*}$-algebras and noncompact quantum groups, Comm. Math. Phys. 136 (1991), no. 2, 399-432, available at http://projecteuclid.org/euclid.cmp/1104202358 MR 1096123

[48] _ Compact quantum groups, Symétries quantiques (Les Houches, 1995), North-Holland, Amsterdam, 1998, pp. 845-884. MR 1616348 
[49] _ From multiplicative unitaries to quantum groups, Internat. J. Math. 7 (1996), no. 1, 127-149, doi: 10.1142/S0129167X96000086 MR 1369908

[50] , Quantum ' $a z+b$ ' group on complex plane, Internat. J. Math. 12 (2001), no. 4, 461-503, doi: 10.1142/S0129167X01000836 MR 1841400

[51] , Quantum E(2) group and its Pontryagin dual, Lett. Math. Phys. 23 (1991), no. 4, 251-263, doi: 10.1007/BF00398822 1152695 (93b:17058)

[52] _, Quantum exponential function, Rev. Math. Phys. 12 (2000), no. 6, 873-920, doi: 10.1142/S0129055X00000344 MR 1770545

[53] Operator equalities related to the quantum E(2) group, Comm. Math. Phys. 144 (1992), no. 2, 417-428, available at http://projecteuclid.org/getRecord?id=euclid.cmp/1104249324 MR 1152380

[54] Simplified quantum E(2) group (2011), unpublished (lecture slides), available at http://www.math tugraz.at/mathc/esi2011/talks

[55] Stanisław Lech Woronowicz and Stanisław Zakrzewski, Quantum 'ax + b' group, Rev. Math. Phys. 14 (2002), no. 7-8, 797-828, doi: 10.1142/S0129055X02001405 Dedicated to Professor Huzihiro Araki on the occasion of his 70th birthday. MR 1932667 